Genetics, epigenetics and angiogenesis in Renal Cell Carcinoma 
Genetics, epigenetics and angiogenesis in Renal Cell Carcinoma

Marcella M.L.L. Baldewijns

ISBN: $\quad 9789052788289$

Cover: Geertjan van Zonneveld

Lay-out: Yvonne Leenders

Printed by Datawyse | Universitaire Pers Maastricht

(C) 2009, Marcella Baldewijns

All rights reserved. No part of this thesis may be reproduced or transmitted in any form or by any means, electronic or mechanical, including photocopying, recording or any information storage or retrieval system, without permission in writing from the author, or, when appropriate, from the publishers of the publications. 


\title{
Genetics, epigenetics and angiogenesis in Renal Cell Carcinoma
}

\author{
PROEFSCHRIFT \\ Ter verkrijging van de graad van doctor \\ aan de Universiteit Maastricht, \\ op gezag van de Rector Magnificus, \\ Prof. mr. G.P.M.F. Mols, \\ volgens het besluit van het College van Decanen, \\ in het openbaar te verdedigen \\ op vrijdag 26 juni 2009 om 12.00 uur
}

door

Marcella Maria Ludovica Lambertina Baldewijns 


\section{Promotor}

Prof. dr. A.P. de Bruïne

\section{Copromotores}

Dr. P. Vermeulen

Dr. M. van Engeland

\section{Beoordelingscommissie}

Prof. dr. F.C. Ramaekers (voorzitter)

Prof. dr. P.A.A. Pauwels, Universitair Ziekenhuis Gent

Prof. dr. J. A. Schalken, Radboud Universitair Medisch Centrum Nijmegen

Prof. dr. V.C.G. Tjan-Heijnen

Prof. dr. Ph. E.V. Van Kerrebroek 


\section{Contents}

Chapter 1 General Introduction $\quad 9$

Chapter 2 Clinical and morphologic characteristics of renal cell carcinoma $\quad 15$

Chapter 3 HIF signalling in Renal Cell Carcinoma angiogenesis 33

Chapter 4 High grade clear cell renal cell carcinoma has a higher angiogenic $\quad 51$ activity than low grade renal cell carcinoma based on histomorphological quantification and qRT-PCR mRNA expression profile.

Chapter 5 Different angiogenic potential in low and high grade sporadic clear cell renal cell carcinoma is not related to alterations in the von Hippel-Lindau gene

Chapter 6 Low frequency of lymph node metastasis in clear cell renal cell carcinoma is related to low lymphangiogenic activity.

Chapter 7 Genetics and epigenetics of renal cell cancer

Chapter 8 Prognostic significance of Gremlin1 (GREM1) promoter CpG island hypermethylation in clear cell renal cell carcinoma

Chapter 9 General Discussion

Summary

Samenvatting

Full colour figures

Dankwoord

About the author 


\section{List of abbreviations}

\begin{tabular}{|c|c|}
\hline ANG & angiopoietin \\
\hline b-FGF & basic-fibroblastic growth factor \\
\hline BHD & Birt-Hogg-Dubé \\
\hline BMP & bone morphogenetic protein \\
\hline CAIX & carbonic anhydrase IX \\
\hline$(\mathrm{cc}) \mathrm{RCC}$ & (clear cell) renal cell carcinoma \\
\hline chrRCC & chromophobe renal cell carcinoma \\
\hline $\mathrm{Cl}$ & confidence interval \\
\hline COX-2 & cyclooxygenase-2 \\
\hline CXCR & chemokine receptor \\
\hline DAC & 5'aza-2-deoxycytidine \\
\hline DNMT & DNA methyltransferase \\
\hline $\mathrm{ECP}(\%)$ & endothelial cell proliferation (fraction) \\
\hline $\mathrm{EGF}(\mathrm{R})$ & epidermal growth factor (receptor) \\
\hline $\mathrm{FH}$ & fumarate hydratase \\
\hline $\mathrm{FIH}$ & factor inhibiting HIF \\
\hline GLUT & glucose transporter \\
\hline GREM1 & gremlin 1 \\
\hline HDAC & histone deacetylases \\
\hline HE & hematoxylin eosin \\
\hline HGF & hepatocyte growth factor \\
\hline HIF & hypoxia-inducible transcription factor \\
\hline HLRCC & hereditary leiomyomatosis renal-cell cancer syndrome \\
\hline HMT & histone methyl transferases \\
\hline HR & hazard ratio \\
\hline HRE & hypoxia response elements \\
\hline HUVEC & human umbilical vein endothelial cells \\
\hline IGF & insulin-like growth factor \\
\hline IFN $\alpha$ & interferon- $\alpha$ \\
\hline IL & interleukin \\
\hline IVD & in vitro methylated DNA \\
\hline LEC & lymph endothelial cells \\
\hline LECP\% & lymph endothelial cell proliferation fraction \\
\hline $\mathrm{LOH}$ & loss of heterozygosity \\
\hline LVA & lymph vessel area \\
\hline LVD & lymph vessel density \\
\hline MBP & methyl binding protein \\
\hline MFS & metastatic free survival \\
\hline
\end{tabular}




$\begin{array}{ll}\text { MMP } & \text { matrix metalloproteinases } \\ \text { MSKCC } & \text { Memorial Sloan-Kettering Cancer Center } \\ \text { MSP } & \text { methylation specific PCR } \\ \text { MVD } & \text { microvessel density } \\ \text { NF-KB } & \text { nuclear factor- } \text { KB } \\ \text { NLCS } & \text { Netherlands cohort series } \\ \text { OS } & \text { overall survival } \\ \text { PBS } & \text { phosphate buffered saline } \\ \text { PDGF(R) } & \text { plateled-derived growth factor (receptor) } \\ \text { PF } & \text { plateled derived factor } \\ \text { PHD } & \text { prolyl hydroxylase } \\ \text { PLGF } & \text { placental growth factor } \\ \text { pRCC } & \text { papillary renal cell carcinoma } \\ \text { ROS } & \text { reactive oxygen species } \\ \text { RT-PCR } & \text { reverse transcriptase PCR } \\ \text { TAM } & \text { tumour-associated macrophages } \\ \text { TCP(\%) } & \text { tumour cell proliferation (fraction) } \\ \text { TGF } & \text { transforming growth factor } \\ \text { TKI } & \text { tyrosine kinase inhibitor } \\ \text { TNF } & \text { tumour necrosis factor } \\ \text { TNM } & \text { tumour-node-metastases } \\ \text { TSA } & \text { Trichostatin A } \\ \text { TSC } & \text { tuberous sclerosis complex } \\ \text { TSG } & \text { tumour suppressor gene } \\ \text { TSP } & \text { thrombospondin } \\ \text { TSS } & \text { urokinase-type plasminogen activator } \\ \text { UPA } & \text { vascular endothelial growth factor (receptor) } \\ \text { VEGF(R) } & \text { VHL }\end{array}$


Chapter 1

General Introduction 


\section{General Introduction}

\subsection{Historical background of renal cancer}

Renal cancer is estimated to account for $2.3 \%$ of all cancer-related deaths in Europe in 2006[1]. Approximately $85 \%$ of all kidney cancers are renal cell carcinomas (RCC), arising from the renal epithelium. RCCs are characterized by a lack of early symptoms, resulting in a quarter of patients with advanced disease, including locally invasive or metastatic RCC at the time of diagnosis. In the management of RCC, surgery has been the most important therapeutic option for localized RCC. Treatment options for metastatic RCC have been limited because of inherent tumour resistance to chemotherapy and radiotherapy. Immunotherapeutic agents, such as interleukin-2 and interferon have shortcomings due to their toxicity and lack of curative responses in most patients. However, recent advances in understanding the biology and genetics of RCC have led to several novel targeted approaches for the treatment of metastatic RCC, with higher response rates.

Renal cell carcinoma (RCC) is not a single entity, but comprises a group of tumours with clinically, histologically and genetically different aspects.

Historically, the earliest confirmed case of RCC was reported by Miriel in 1810[2]. In 1826 the first classification of renal tumours was proposed by König. This classification was based on macroscopic morphology, dividing renal tumours into scirrhous, steatomatous, fungoid and medullary forms[3]. The histogenesis of RCC was controversial for a long time. Kupfer suggested in 1865 that renal tumours derived from the malignant transformation of intrarenal Wolffian rests. However, Waldeyer thought that the tumour originated from renal tubular epithelium, which was supported by Klebs in 1876 and Lancereaux in 1889[4]. Nevertheless, a debate was initiated in 1883, when Virchow and Grawitz postulated that these tumours were derived from ectopic adrenocortical elements in the renal tissue. The morphology of the tumour, representing as yellow predominantly subcapsular tumours[5] and the frequent location in the upper pole of the kidney, led to the erroneous belief that renal tumours originated from misplaced adrenal tissue. Grawitz consolidated his theory in 1884 with further illustrations of intrarenal ectopic adrenal tissue[6]. Grawitz's theory was widely accepted and for many years these tumours were called hypernephromas[7,8].For 14 years, the debate about the histogenesis of renal carcinoma persisted, with the majority of reports favouring the adrenal rest origin, although some authors, such as Kelynack, still believed that these tumours were of renal epithelial origin[9].

Vigorous criticism of Grawitz was provided by Stoerk in 1908, who considered the adrenal origin of the renal tumours unproven. By comparing the relative frequency of renal tumours with the scarcity of malignant epithelial tumours of the adrenal gland he commented on the lack of similarity between hypernephroma and adrenal carcinoma[10]. Despite these compelling arguments, the term hypernephroma, with its associated adrenal connotation, persisted in the literature. 
The debate was finally settled by convincing evidence of Oberling et al. in 1959 who studied the ultrastructure of clear cells from eight renal carcinomas. They found that the tumour cell cytoplasm contained numerous mitochondria and deposits of glycogen and fat and identified cytoplasmic membranes inserted perpendicularly onto the basement membrane with occasional cells containing microvilli along the free borders. These features indicated that the tumours arose from the epithelial cells of the renal convoluted tubule. By this, one of the most debated issues in tumour pathology was ended.

Over the years, many investigators have attempted to establish grading schemes for RCC, but the grading system proposed by Fuhrman et al.[11] in 1981 is still the most widely used in clinical practice. This four-tiered classification is based on nuclear morphology (nuclear size, irregularity of the nuclear membrane and nucleolar prominence) and showed good correlation with clinical outcome[11].

RCCs have historically been classified according to cell type (clear, granular, spindle or oncocytic) and growth pattern (acinar, papillary or sarcomatoid). This classification gave little insight into the clinical behavior of the carcinoma. A new histopathological classification of renal tumours was proposed in 1986, based on morphological, histochemical and electron-microscopical data[12] and subsequently validated by cytogenetic and molecular studies[13-16]. During the past twenty years, studies of families with inherited RCC have laid the groundwork for the identification of hereditary RCC syndromes. The identification of genes involved in familial renal neoplasms has provided insight into the genetic basis of sporadic RCCs as well. The year 1993 marked the discovery of the von Hippel-Lindau (VHL) tumour suppressor gene[17], the first gene identified for hereditary RCC that is now known to be involved in most cases of sporadic RCC. Four years later, mutations in the MET proto-oncogene were identified in patients with hereditary papillary RCC[18] and in 2002 the gene that encodes the Krebs cycle enzyme fumarate hydratase (FH) was found to be mutated in renal tumours from patients with a rare genodermatosis termed hereditary leiomyomatosis and RCC[19]. Finally, new renal-cancer-predisposing genes were identified through linkage analysis in families with another genodermatosis, the Birt-Hogg-Dubé syndrome[20] and in families with hyperparathyroidism-jaw tumour syndrome[21].

The extensive histological and molecular evaluation of RCC has resulted in the development of a consensus classification, which was proposed on an international workshop in Heidelberg in 1996[22]. This classification included different types of RCC, each with a different histology and caused by different gene alterations (Table 1).

The surprisingly diverse nature of gene alterations in RCC subtypes implicates various mechanisms and biological pathways in RCC tumorigenesis.

Over the past decades, the cloning of susceptibility genes that underline the familial predisposition has offered entry points into the signalling pathways that are deregulated in sporadic RCC with concomitant identification of potential therapeutic targets. However, characteristics predicting the clinical behaviour and response to treatment are still lacking. 
Table 1. Heidelberg classification of RCC

\begin{tabular}{lll}
\hline Histological type & Genes implicated & Chromosomal abnormalities \\
\hline Clear cell RCC & VHL, BHD & $-3 p,+5 q,-Y,-8 p,-9 p,-14 q ; t(3 ; 5)(p ; q)$ \\
Papillary RCC & MET,FH,HRPT2 & $+7,+17, Y,+12,+16,+20$ \\
& & $t(X ; 1)(p 11.2 ; q 21.2) t(X ; 17)(p 11.2 ; q 25.3)$ \\
Chromophobe RCC & BHD & $-1,-2,-6,-10,-13,-17,-21$ \\
Collecting-duct carcinoma & FH & $-1 q 32,-6 p,-8 p,-21 q$ \\
\hline VHL: von Hippel-Lindau; BHD: Birt-Hogg-Dubé: FH: Fumarate hydratase; HRPT2: hyperparathyroidism 2[23]
\end{tabular}

\subsection{Hypothesis and outline of this thesis}

The highly vascularized phenotype of ccRCC suggests that angiogenesis is integral to its pathogenesis and clinical course.

The hypothesis of this thesis was that differences in angiogenesis/lymphangiogenesis may explain variable tumour behaviour within the ccRCC subtype. Furthermore, we hypothesized that differences in angiogenic activity might be explained by different alterations in the $\mathrm{VHL}$ gene, a common event in ccRCC tumourigenesis and key player in HIF driven angiogenesis. If angiogenic behaviour in $\mathrm{CCRCC}$ is not directly influenced by VHL alterations, this suggests that additional HIF downstream events may affect tumour driven angiogenesis. To that end, we focused on the importance of epigenetic changes in ccRCC and additionally looked for epigenetically silenced tumour suppressor genes, which were involved in angiogenesis. With regard to this, we investigated the influence of GREM1 promoter hypermethylation on angiogenesis/tumour behaviour and clinical outcome.

Introductory to the first experimental study, we reviewed the clinical presentation of RCC, including epidemiology, risk factors, classification, prognosis and therapy (chapter 2) and highlighted the current status and future perspectives of angiogenesis research in RCC (chapter 3). In chapter 4 we compared angiogenic activity between high grade and low grade ccRCC. A panel of antibodies was used for immunohistochemistry: CD34/Ki-67, carbonic anhydrase IX (CAIX), hypoxia-inducible factor-1 $\alpha$ (HIF-1 $\alpha)$ and vascular endothelial growth factor (VEGF). Vessel density (MVD), endothelial cell proliferation fraction (ECP\%) and tumor cell proliferation fraction (TCP\%) were assessed. mRNA expression levels of angiogenesis stimulators and inhibitors were determined by quantitative RT-PCR.

Subsequently, we investigated the relationship between VHL alterations and angiogenesis/tumour behaviour in CCRCC, by means of immunohistochemistry (angiogenesis/tumour parameters), direct sequencing (VHL mutation status) and methylationspecific PCR (MSP) (chapter 5).

Chapter 6 comprises the assessment of lymphangiogenesis in ccRCCs and impact on lymph node metastases. Double staining with Ki67 and D2-40 was used to assess active lymphangiogenesis and mRNA expression levels of lymphangiogenic factors were determined by real-time quantitative RT-PCR. 
In Chapter 7 we reviewed not only the genetical background of RCC, but also highlighted the evolving research on epigenetical events in RCC with concomitant clinical implications.

In chapter 8, we described gremlin (GREM1) gene silencing via promoter hypermethylation in a group of ccRCCs and investigated the relationship between GREM1 promoter hypermethylation and angiogenesis/tumour parameters, by means of immunohistochemistry (angiogenesis/tumour parameters) and GREM1 promoter methylation analysis (MSP) and bisulfite sequencing.

Finally, in chapter 9 the experimental results, described in this thesis, and their possible implications are discussed, and future directions are given. 


\section{References}

1 Ferlay, J. et al. (2007) Estimates of the cancer incidence and mortality in Europe in 2006. Ann Oncol 18, 581-592

2 Miriel, G. Summarized thoughts on the importance of diagnosis., (Paris, 1810).

3 Delahunt, B. and Eble, J.N. (2005) History of the development of the classification of renal cell neoplasia. Clin Lab Med 25, 231-246, v

4 Ewing, J. Neoplastic diseases, (Philidelphia, 1919).

5 Grawitz, P.A. (1883) Die sogenannten lipomen der niere. Arch Path Anat Physiol 93, 39-63

6 Grawitz, P.A. (1884) Die entstehung von nierentumoren aus nebennierengewebe. Arch Klin Chir 30, 824-834

7 Birch-Hirschfeld, F.V. (1898) Bieträge zur pathologischen anatomie der Nierengeschwuelste. Beit Pathol Anat Alleg Pathol 24, 343-414

8 Lubarsch, O. (1894) Über die abstammung gewisser Nierengeschwuelste von embryonal abgesprengten Nebennierenkeimen. Arch Pathol ana Phusiol Klin Med 137, 191-196

9 Kelynack, T.N. Renal growths.

10 Stoerk, O. (1908) Zur histogenese der Grawitz'chen Nierengeschuelste. Beit Pathol Anat Alleg Pathol 43, 393-437

11 Fuhrman, S.A. et al. (1982) Prognostic significance of morphologic parameters in renal cell carcinoma. Am J Surg Pathol 6, 655-663

12 Thoenes, W. et al. (1986) Histopathology and classification of renal cell tumors (adenomas, oncocytomas and carcinomas). The basic cytological and histopathological elements and their use for diagnostics. Pathol Res Pract 181, 125-143

13 van den Berg, E. et al. (1993) Cytogenetic analysis of epithelial renal-cell tumors: relationship with a new histopathological classification. Int J Cancer 55, 223-227

14 Weiss, L.M. et al. (1995) Adult renal epithelial neoplasms. Am J Clin Pathol 103, 624-635

15 Storkel, S. et al. (1989) The human chromophobe cell renal carcinoma: its probable relation to intercalated cells of the collecting duct. Virchows Arch B Cell Pathol Incl Mol Pathol 56, 237-245

16 Speicher, M.R. et al. (1994) Specific loss of chromosomes 1, 2, 6, 10, 13, 17, and 21 in chromophobe renal cell carcinomas revealed by comparative genomic hybridization. Am J Pathol 145, 356-364

17 Latif, F. et al. (1993) Identification of the von Hippel-Lindau disease tumor suppressor gene. Science 260, 1317-1320

18 Schmidt, L. et al. (1997) Germline and somatic mutations in the tyrosine kinase domain of the MET proto-oncogene in papillary renal carcinomas. Nat Genet $16,68-73$

19 Tomlinson, I.P. et al. (2002) Germline mutations in FH predispose to dominantly inherited uterine fibroids, skin leiomyomata and papillary renal cell cancer. Nat Genet 30, 406-410

20 Nickerson, M.L. et al. (2002) Mutations in a novel gene lead to kidney tumors, lung wall defects, and benign tumors of the hair follicle in patients with the Birt-Hogg-Dube syndrome. Cancer Cell 2, 157-164

21 Carpten, J.D. et al. (2002) HRPT2, encoding parafibromin, is mutated in hyperparathyroidism-jaw tumor syndrome. Nat Genet 32, 676-680

22 Kovacs, G. et al. (1997) The Heidelberg classification of renal cell tumours. J Pathol 183, 131-133

23 Pavlovich, C.P. and Schmidt, L.S. (2004) Searching for the hereditary causes of renal-cell carcinoma. Nat Rev Cancer 4, 381-393 


\section{Chapter 2}

\section{Clinical and morphologic characteristics of renal cell carcinoma}

Marcella M.L. Baldewijns, Iris J.H. van Vlodrop, Leo J. Schouten, Patricia M.M.B.

Soetekouw, Adriaan P. de Bruïne and Manon van Engeland

Biochim Biophys Acta. 2008 Apr;1785(2):133-55. Review (first part). 


\begin{abstract}
Renal cell carcinomas (RCCs) are responsible for 80 to $85 \%$ of all primary renal neoplasms and comprise a group of tumours with different histology and clinical course, responding differently to therapy.

The incidence of RCC has increased over time and varies widely from region to region. A number of environmental and clinical factors has been implicated in the aetiology of RCC.

Slow improvement in prognosis has been observed over time. Currently, the most used prognostic factors for patients with RCC are performance status, tumour stage and Fuhrman grade. Prognostic systems, such as the Memorial Sloan-Kettering Cancer Center system, has enabled better clinical trial design both in the adjuvant, as well as the advanced metastatic disease setting. For patients with early stage disease, radical nephrectomy is the treatment of choice. However $30 \%$ of these patients will relapse and develop future metastasis. Metastatic RCC has historically been refractory to cytotoxic and hormonal agents. Only interleukin- 2 and interferon- $\alpha$ provide response in a minority of patients. Better understanding of RCC biology and underlying oncogenic pathways has led to development of novel, effective targeted therapies.
\end{abstract}




\section{Descriptive epidemiology}

In 2002, worldwide about 210,000 cases of kidney cancer have been diagnosed and 102,000 persons died because of kidney cancer[1]. In the European Union and in the United States, kidney cancer is the 9th most common cancer. Incidence and mortality rates are approximately twice as high for men as for women[1].

Incidence rates for renal cell cancer have been rising steadily each year in Europe and the United States over the past three decades[2]. This increase cannot be explained completely by the observed decrease in mean tumour size, which might indicate improved detection[3], since an increasing incidence of late-stage renal cell cancers also has been observed[2,4]. Rates of renal cancer vary internationally more than tenfold, suggesting a strong role for environmental risk factors. Incidence is generally highest in several Western and Eastern European countries and Scandinavia, as well as in parts of Italy, in North America and in Australia/New Zealand. The lowest rates are reported in Asia and Africa[1].

\section{Risk factors}

Several factors have been investigated in association with the risk of renal cell carcinoma (RCC). Some risk factors, such as cigarette smoking[5-8], obesity[5,6,8,9], hypertension and/or use of antihypertensive medication[7,10,11] were consistently reported to be positively associated with RCC risk, although risk ratios were only moderately increased. A recent meta-analysis on cigarette smoking in relation to RCC concluded that inhaled tobacco smoke is clearly implicated in the etiology of RCC. Risk was increased for former (RR: $1.21 ; 95 \% \mathrm{Cl}: 1.07-1.36$ ) and current (RR: $1.45 ; 1.26-1.66)$ smokers with a strong dose-dependent increase in risk[12]. Most studies observed increasing risks, not only with the number of cigarettes smoked per day but also with the number of years smoked and decreasing risks with number of years since cessation, as is also shown in a meta-analysis by Hunt et al.[12].

A positive association between body weight and risk of renal cell cancer has been observed in almost all case-control and cohort studies[7]. In a quantitative review, a cumulative risk per $1 \mathrm{~kg} / \mathrm{m} 2$ increase in $\mathrm{BMI}$ of 1.07 (95\% Cl: $1.05-1.09)$ was found for men and women[13]. The attributable proportion of RCC for excess body weight is estimated to be 25 percent for both men and women in the European Union[14]. Hypertension and antihypertensive medication have been found to be risk factors for RCC in many epidemiological studies[10,11]. In a meta-analysis[10] based on 13 casecontrol studies a pooled adjusted odds ratio of $1.75(95 \% \mathrm{Cl}, 1.61-1.90)$ was calculated for the association between hypertension and RCC. In another meta-analysis, a pooled odds ratio was calculated for use of diuretics and risk of RCC[11]. Based on nine casecontrol studies, an average odds ratio was calculated of 1.55 (95\% Cl, 1.42-1.71). It is unclear, whether the increased risk is caused by hypertension itself, or by the use of 
antihypertensive medication. Some recent studies showed that diuretic medication is no longer a risk factor after controlling for the diagnosis of hypertension[15,16], suggesting that not medication but hypertension is a risk factor for RCC. It is also conceivable that hypertension acts as biomarker for another disease that might be causally associated with RCC.

Furthermore, dialysis patients with acquired cystic disease of the kidney showed a 30 times greater risk than in the general population for developing RCC[17]. Whether prolonged ingestion of analgesic combinations (phenacetin, aspirin), leading to chronic renal failure, enhances the incidence of RCC remains controversial[18-20].

Several studies have been conducted investigating whether occupational exposures are associated with RCC risk. Exposure to asbestos, polycyclic aromatic hydrocarbons, gasoline have not been shown to be consistently associated with RCC risk[21]. Interestingly, occupational exposure to trichloroethylene has been shown to be associated with specific mutations in the VHL-gene in RCC $[22,23]$.

Reports on other factors showed less consistent associations with RCC risk. These factors include alcohol consumption, vegetable and fruit consumption, intake of micronutrients and vitamins $[5,6,8,9]$. Most studies on alcohol consumption and RCC risk observe null associations[24-30] while some more recent studies suggest an inverse association[31-34], although this effect may be limited to women[35]. In a recent, pooled analysis of 12 prospective cohort studies, intake of alcohol was associated with a decreased risk of renal cell cancer. Compared to non-drinkers, alcohol consumption of $\geq 15$ grams/day was associated with a pooled RR of 0.72 (95\% Cl: 0.60-0.86)[36].

Finally, patients with certain inherited disorders such as von Hippel-Lindau disease, hereditary papillary renal cancer, a hereditary leiomyoma RCC syndrome and BirtHogg-Dube syndrome, show an enhanced risk of RCC[37-39]. These inherited disorders show specific genetic abnormalities (see further chapter 7).

\section{Classification}

The majority of kidney cancers (80-85\%) are RCCs originating from the renal parenchyma. The remaining $15-20 \%$ are mainly transitional cell carcinomas of the renal pelvis. Other less frequent malignant tumours of the kidney are metanephric adenosarcomas, mesenchymal tumours, mixed mesenchymal and epithelial tumours, neuroendocrine tumours, hematopoietic and lymphoid tumours, germ cell tumours and metastatic tumours. Though nephroblastomas (Wilms tumour) are the most common renal tumours of childhood, few cases have been reported in adulthood[40-42].

RCC is almost exclusively a cancer of adults and is rare in the first two decades of life, comprising only $2 \%$ of pediatric renal tumours.

RCC is not a single entity (see figure 1), but comprises a group of tumours, which arise from the epithelium of renal tubules. Extensive histological and molecular evaluation has resulted in the development of a consensus classification of different RCC subtypes. 


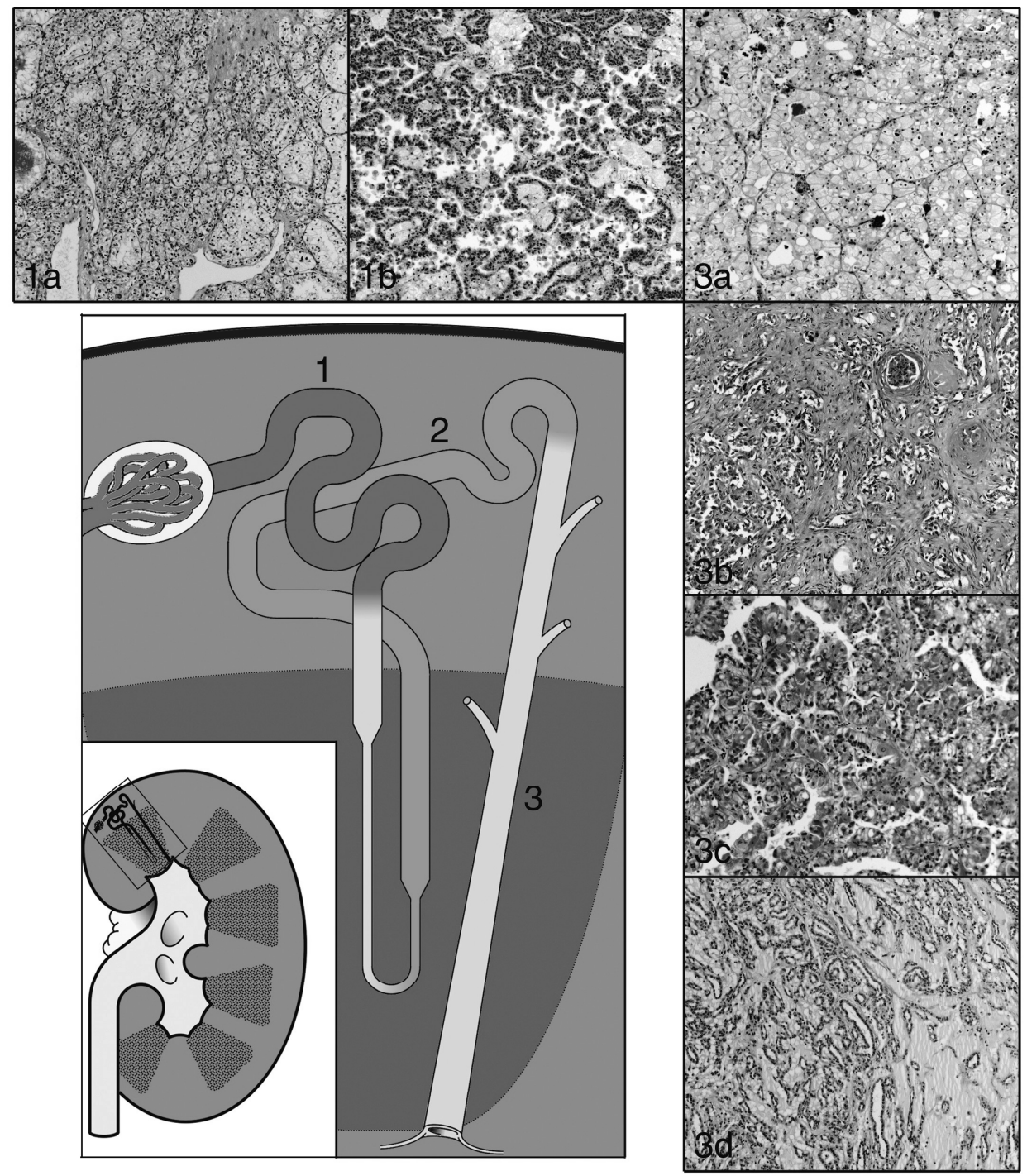

Figure 1: Classification of RCC subtypes: cell of origin

The nephron, consists of the renal corpuscle (1) and renal tubule, which is divided into four histophysiological zones: proximal convoluted tubule (2), loop of Henle (3), distal convoluted tubule (4) and collecting tubule (5). The different RCC subtypes are thought to originate from different parts of the renal tubular system: mainly the proximal convoluted tubule ( $2 a$ clear cell RCC and $2 b$ papillary RCC) and the collecting tubule (5a chromophobe RCC, 5b collecting duct carcinoma, and 5c mucinous tubular and spindle cell carcinoma). For full colour picture see page 162.

In 1996, the Heidelberg classification of RCC was proposed, which intends to integrate the understanding of genetic alterations and readily recognizable histological criteria[43]. In this classification, three major histological subtypes of RCC, clear cell (ccRCC), papillary (pRCC) and chromophobe (chrRCC) RCC have been identified in addition to some less frequent subtypes such as multilocular ccRCC, collecting duct carci- 
nomas, medullary carcinomas and unclassified types. The latest WHO classification of 2002 is more comprehensive and includes, in addition to the previous subtypes, renal carcinoma associated with neuroblastoma and two recent added subtypes, the Xp11 translocation carcinomas and mucinous tubular and spindle cell carcinoma (Table 1).

Table 1: Histological subtypes of RCC (WHO classification, 2002)

\begin{tabular}{lllll}
\hline & Frequency (\%) & Age & $\begin{array}{l}\text { Male/female } \\
\text { ratio }\end{array}$ & Ref. \\
\hline Clear cell renal cell carcinoma & 70 & $61^{\text {a }}$ & & {$[43]$} \\
Papillary renal cell carcinoma & $10-15$ & $61^{\text {a }}$ & $1.8 / 1$ to 3.8/1 & {$[54-57]$} \\
Chromophobe renal cell carcinoma & 5 & $60^{\text {a }}$ & $1 / 1$ & {$[49-52]$} \\
Renal cell carcinoma unclassified & $4-5$ & & & {$[54,62]$} \\
Multilocular clear cell carcinoma & $<5$ & $51^{\text {a }}$ & $3 / 1$ & {$[53]$} \\
Carcinoma of the collecting ducts of Bellini & $<1$ & $55^{\text {a }}$ & $2 / 1$ & {$[59,60]$} \\
Renal medullary carcinoma & $<1$ & $22^{\text {a }}$ & $2 / 1$ & {$[61]$} \\
Xp11 translocation carcinomas & $<1$ & $15^{\text {b }}$ & $4 / 7$ & {$[63,64]$} \\
Carcinoma associated with neuroblastoma & $<1$ & $13.5^{\text {b }}$ & $1 / 1$ & {$[66]$} \\
Mucinous tubular and spindle cell carcinoma & $<1$ & $53^{\mathrm{a}}$ & $1 / 4$ & {$[65]$} \\
\hline
\end{tabular}

${ }^{a}$ mean age (years), ${ }^{b}$ median age (years)

Clear cell carcinoma (70\% of all RCCs) and pRCC (10-15\%) account for the majority of RCCs. They are thought to arise from cells in the proximal convoluted renal tubule[38,43,44]. Most cases of ccRCC are unilateral and unifocal. Invasion of perirenal and sinusoidal fat and/or extension into the renal vein occurs in about $45 \%$ of cCRCC[45]. ccRCC most commonly metastasize hematogeneously via the vena cava primarily to the lung, although in $7 \%$ to $17 \%$ lymphatic metastases occur[46,47]. In $5 \%$ of ccRCCs a predominantly cystic growth pattern is seen. These tumours are called multilocular cystic RCCs. Multilocular cystic RCCs mostly present with low Fuhrman grade and stage at diagnosis and display a favourable outcome, suggesting a tumour with low malignant potential[48-53]. Though no recurrences or metastasis have been reported, additional outcome based research is needed because of the rarity of this subtype and the limited number of patients reported so far in the literature. pRCC is subclassified in two subtypes depending on the morphology of the tumour cells covering the papillary lesions[54]. Type 1 tumours have papillae covered by a single layer of small cells with scant cytoplasm, whereas in type 2 tumours the papillary cores are covered by pseudostratified epithelium with a larger amount of eosinophilic cytoplasm and often higher nuclear grade. Up to $70 \%$ of pRCCs are intrarenal at diagnosis[55,56]. Type 1 tumours are usually of lower grade and stage than type 2 tumours[56,57]. Bilateral and multifocal tumours are more common in PRCC than in the other malignant subtypes The chromophobe variant of RCC accounts for 5 percent of all cases of RCC and is thought to originate from type B intercalated cells of renal collecting ducts[44,58]. Only $10 \%$ of chrRCC show extension through the renal capsule into the surrounding adipose tissue and in only $4 \%$ there is involvement of the renal vein[58]. Collecting duct (Bellini duct) carcinomas are thought to arise from the collecting ducts within the renal medulla[44,59]. These tumours may present at any age, although they tend to occur in younger patients than the former subtypes. These tumours are very 
aggressive, one third exhibiting metastases at the time of presentation. Approximately two-thirds of patients die of this disease within two years of diagnosis[60]. A variant of the collecting duct carcinoma is the so-called medullary carcinoma[61], which develops preferentially in young patients with sickle trait. These tumours are now widely regarded as a more aggressive variant of collecting duct carcinoma, with a mean survival time after surgery of 15 weeks[60]. The unclassified RCCs are a diagnostic category of renal tumours which do not fit into one of the other subtypes. In surgical series, this group often amounts to $4-5 \%$ of all cases[43,44]. Many of the tumours from this category are high cyto-morphologic grade and show aggressive clinical behaviour[62]. Translocation-associated RCC affect predominantly children and young adults and present at advanced stage[63,64]. Mucinous tubular and spindle cell carcinoma is recently identified as a novel entity of low-grade renal epithelial neoplasm, characterized by an indolent course and, in contrast with the other subtypes, female predominance. Its immunohistochemical and ultrastructural features are consistent with distal nephron origin[65]. Finally, RCC associated with neuroblastoma occurs in long-term survivors of childhood neuroblastoma, by which males and females are equally affected. These RCCs are morphologically heterogeneous and prognosis correlates with tumor grade and stage[66].

Most cases of RCC occur sporadically. An inherited predisposition to renal cancer accounts for only $1-4 \%$ of all cases[38]. Inherited renal neoplasms are more likely to be diagnosed at an younger age and are more likely to be multifocal and bilateral.

\section{Prognosis}

A gradual improvement in prognosis has been observed over time, with 5-year relative survival rates for RCC as high as $64 \%$ in 2002 , compared with less than $40 \%$ in the early 1960s[67]. The clinical behaviour of RCC results from complex interactions between multiple prognostic factors. Currently, patient performance status, tumour stage and grade are the most useful, clinical available predictors of patient outcome[68-70]. The performance status is measured using the Eastern Cooperative Oncology Group (ECOG) assessment scale. This scoring system stratifies patients on the basis of ambulatory status (rated 0-5), in order to denote the impact of disease on the overall health of the patient[71].

Currently, the most commonly used staging system is the International Union Against Cancer (IUAC) and the American Joint Committee on Cancer (AJCC) tumor-nodemetastases (TNM) staging system. The 2002 modification of this system (sixth edition) has recently been confirmed to have a better prognostic ability than the previous 1997 staging system [72]. Using the 2002 staging system, the 5-year cancer specific survival rates ranged from $97 \%$ for pT1A to $20 \%$ for pT4. Presence of lymph node metastases predicts a poor prognosis, with cancer-specific survival rates of $5-30 \%$ and $0-5 \%$ at 5 and 10 years, respectively[73]. Metastases to other organs (lung, brain, bone) are as- 
sociated with survival rates of $50 \%, 5-30 \%$ and $0-5 \%$ at 1,5 and 10 years respectively. Grading is based on the Fuhrman system, which consists of four grades, based on the nuclear size, contour and conspicuousness of nucleoli[74].

Kim et al. examined a cohort of 1,046 patients with localized and metastatic RCC and identified hypoalbuminemia, weight loss, anorexia and malaise as independent predictors of poor prognosis[75]. The presence of thrombocytosis (platelet count > $400,000 / \mathrm{mm}^{3}$ ) has been shown to be an independent predictor of poor outcome in patients with metastatic RCC treated with nephrectomy and adjuvant therapy[76]. Whether different histological RCC subtypes have different clinical behaviour and outcome remains a topic of discussion. Some reports demonstrated worse outcome for cCRCC compared with pRCC and chrRCC $[77,78]$. However, in multivariate analyses, TNM stage, Fuhrman grade and ECOG, but not histology, were retained as independent prognostic variables[69]. Tumour necrosis was found to be a strong independent predictor of poor outcome for clear cell but not for chromophobe or papillary RCC $[79,80]$. Micro-vascular invasion and presence of sarcomatoid features are reported negative prognostic indicators[81,82]. Since multiple factors affect prognosis, many centers have aimed to integrate independent prognostic indicators into comprehensive outcome models for both nonmetastatic and metastatic RCC to assist clinicians in patient counseling, management decisions and also for clinical trial comparisons[80,83-94].

The most widely accepted prognostic system is the Memorial Sloan Kettering system for clinical trial assessment and treatment assignment. This model was based on a study of 670 patients with advanced RCC treated in 24 separate clinical trials at Memorial Sloan-Kettering Center (MSKCC; New York, NY) between 1975 and 1996[92]. Five prognostic variables for short survival were selected by univariate and multivariate analysis: low performance status (Karnofsky $<80 \%$ ), high serum lactate dehydrogenase (> 1.5 times the upper limit of normal), low hemoglobin (less than the lower limit of normal), high corrected serum calcium ( $>10 \mathrm{mg} / \mathrm{dL}$ ), and absence of prior nephrectomy. Patients were stratified into favourable-, intermediate-, and poor-risk groups according to the number of risk factors present. A subsequent analysis was performed in 463 patients treated with interferon alfa as first-line systemic therapy, the MSKCC model remained the same except time from diagnosis to treatment with IFN $\alpha$ of less than 1 year was found to be a better predictor of short survival compared with absence of prior nephrectomy[91]. In a more recent study of 251 previously treated patients with metastatic RCC, pre-treatment features associated with shorter survival after multivariate analyses (low Karnofsky performance status, low hemoglobin level, and high corrected serum calcium) were used as risk factors to categorize patients into favourable risk group (zero risk factor, median survival 22 months), intermediate-risk group (one risk factor, median survival 11.9 months) and poor risk group (two or three risk factors, median survival 5.4 months)[95]. These risk categories are currently used in clinical trial design of phase III trials for stratification and patient counselling. 
Recent identification and incorporation of molecular markers into current staging systems may result in better prognostic tools. By measuring protein expression or gene copy number, several molecular factors have been identified as independent risk factors for developing RCC, including B7-H1, Smac/DIABLO, survivin, PTEN, p27 and VEGF[96-100]. Other reported prognostic indicators are HIF-1 $\alpha$, CAIX, Ki 67, gelsolin, vimentin, p53 and EphrinA2[101-103]. Until now, there have been no molecular markers for RCC that meet the College of American Pathologists criteria for a marker to be used generally in patient management or even to have been studied sufficiently biologically to provide for any degree of acceptance[104].

\section{Therapy}

Current treatments of patients with RCC can be divided in local therapy and systemic therapy.

\subsection{Local therapy}

For patients with localized tumours, radical nephrectomy is currently the therapy of choice for tumours larger than four centimeters, regardless of histological subtype[105]. Partial nephrectomy has gained acceptance for treating tumours smaller than $4 \mathrm{~cm}$ in diameter or indications such as solitary kidney, bilateral renal tumour localization, renal insufficiency as well as the presence of hypertension, diabetes or hereditary renal cell carcinoma syndromes[105].

In 20-25\% of patients, metastases are present at the time of diagnosis of the disease. Based on the results of two randomized studies, nephrectomy is standard as cytoreductive treatment before embarking on systemic therapy with cytokines with a survival advantage of 3[106] to 10 months[107]. However, since high dose interleukin-2 (IL-2) can be effective in patients with high metastatic burden and a relatively small primary tumour, some groups suggest that nephrectomy should not be offered in this group of patients[108]. In patients who will be treated with targeted therapy, the role of cytoreductive surgery is not yet known and needs to be reassessed.

The response rate of RCC to radiotherapy is $50 \%$ and it may be useful as palliative therapy in patients who are not eligible for surgery or in case of bone or central nervous system metastases[109].

\subsection{Systemic therapy}

Hormonal and chemotherapy are not standard treatment in RCC because response rates of these agents are low[110]. Drug resistance may be related to the expression of the multidrug resistance transporter in proximal tubule cells, from which ccRCC and pRCC originate[111,112]. For more than 20 years, immunotherapy has been the leading treatment for metastatic RCC. Prolonged stabilization of disease in the absence of 
systemic therapy and rare spontaneous regressions suggest that host immune mechanisms play a role in tumour growth regulation[113]. Therefore, RCC became attractive to test various immunologic manipulations, e.g. IL-2, interferon- $\alpha$ (IFN $\alpha$ ), and two (still) experimental treatments (stem cell transfusions from HLA-compatible siblings following immunoablative chemotherapy[114] and tumour vaccines[115]).

IL-2 and IFN $\alpha$ were first reported to have anti-tumour activity. Approximately 5 to 15\% of cases of metastatic CCRCC respond to IFN $\alpha$, however most of these responses are partial or of short duration[116,117]. Treatment with IL-2, the current Food and Drug Administration (FDA)-approved agent for patients with advanced renal carcinoma is associated with a complete response in $10 \%$ and partial response in $11 \%$ of patients[61]. Given the toxicity, expense and variable response to IL-2 among patients with metastatic RCC, several clinical, histological and molecular response predictors have been evaluated[108,118]. Cytokine based therapy currently remains an option for patients with metastatic ccRCC and good prognosis according to MSKCC-risk classification[119].

Studies with targeted agents have been recently reported and some of them are now considered as standard treatment on the basis of proven efficacy in Phase III trials. Therefore, Sunitinib, Sorafenib and Temsirolimus were recently approved by the US FDA for treatment of metastatic RCC and are recurrently included in clinical practice.

Sunitinib (SU11248) is a multi-targeted tyrosine kinase inhibitor (TKI) that inhibits the receptor tyrosine kinases VEGFR2, PDGFR, FMS-like tyrosine kinase 3 (FLT-3) and c-KIT. Sunitinib was the first therapy to demonstrate superiority over standard cytokine therapy (IFN $\alpha$ ) as first line treatment in 750 patients with mainly good and intermediate prognosis according to MSKCC-risk classification[120]. This drug was given orally once daily in a dose of $50 \mathrm{mg}$ followed by 2 weeks without treatment. Progression free survival (PFS) increased from 5 to 11 months and partial response by Response Evaluation Criteria in Solid tumors (RECIST) criteria was $37 \%$ for Sunitinib compared to $9 \%$ for IFN $\alpha$. Overall survival difference has not yet reached statistical significance.

Another oral multi-targeted TKI is Sorafenib (BAY 43-9006) which inhibits the nonreceptor serine threonine kinases $B R A F$ and $C R A F$ and the receptor tyrosine kinases VEGFR2, VEGFR3, FLT-3, c-KIT and PDGFR. After failure of first line treatment Sorafenib (given orally 2 times daily in a dose of $400 \mathrm{mg}$ ) showed clinical benefit as compared to placebo in patients with intermediate risk or low risk status according to MSKCCprognostic score[121]. PFS prolonged from 3 to 6 months and PR was $10 \%$ compared to $2 \%$ in the placebo group. There is a difference in the curves of overall survival in this study which is not yet statistically significant. Temsirolimus is an inhibitor of the nonreceptor tyrosine kinase mTOR which revealed activity against metastatic RCC with MSKCC-poor risk characteristics[122]. As compared with IFN $\alpha$ and IFN $\alpha$-Temsirolimus combination therapy, Temsirolimus monotherapy (given intravenously weekly in a dose of $25 \mathrm{mg}$ ) improved overall survival from respectively 7.3 and 8.4 months, to 10.9 months. The objective response rates were not significantly different between the three groups. An additional drug in the near future may be Bevacizumab, a monoclonal 
antibody directed against VEGF. Compared to placebo this drug improved PFS[123]. Results of two randomized trials comparing a combination of IFN $\alpha$ with Bevacizumab with IFN $\alpha$ monotherapy or with a placebo are underway. 


\section{References}

1 Ferlay, J. et al. GLOBOCAN 2002: Cancer incidence, mortalitity and prevalence worldwide, (IARCPress, Lyon, 2004).

2 Mathew, A. et al. (2002) Global increases in kidney cancer incidence, 1973-1992. Eur J Cancer Prev 11, 171-178

3 Nguyen, M.M. et al. (2006) The evolving presentation of renal carcinoma in the United States: trends from the Surveillance, Epidemiology, and End Results program. J Urol 176, 2397-2400; discussion 2400

4 Hock, L.M. et al. (2002) Increasing incidence of all stages of kidney cancer in the last 2 decades in the United States: an analysis of surveillance, epidemiology and end results program data. J Urol 167, 57-60

5 Dhote, R. et al. (2000) Risk factors for adult renal cell carcinoma: a systematic review and implications for prevention. BJU Int 86, 20-27

6 Lindblad, P. (2004) Epidemiology of renal cell carcinoma. Scand J Surg 93, 88-96

7 McLaughlin, J.K., Lipworth, L., Tarone, R.E., Blot, W.J. Cancer epidemiology and prevention, (Oxford University Press, Oxford, 2006).

8 Mellemgaard, A. (1999) Human renal-cell carcinoma--epidemiological and mechanistic aspects. IARC Sci Publ, 69-80

9 Wolk, A. et al. (1996) Nutrition and renal cell cancer. Cancer Causes Control 7, 5-18

10 Grossman, E. et al. (2002) Is there an association between hypertension and cancer mortality? Am J Med 112, 479-486

11 Grossman, E. et al. (1999) Does diuretic therapy increase the risk of renal cell carcinoma? Am J Cardiol 83, 1090-1093

12 Hunt, J.D. et al. (2005) Renal cell carcinoma in relation to cigarette smoking: meta-analysis of 24 studies. Int J Cancer 114, 101-108

13 Bergstrom, A. et al. (2001) Obesity and renal cell cancer--a quantitative review. Br J Cancer 85, 984-990

14 Bergstrom, A. et al. (2001) Overweight as an avoidable cause of cancer in Europe. Int J Cancer 91, 421430

15 McLaughlin, J.K. et al. (1995) International renal-cell cancer study. VIII. Role of diuretics, other antihypertensive medications and hypertension. Int J Cancer 63, 216-221

16 Shapiro, J.A. et al. (1999) Hypertension, antihypertensive medication use, and risk of renal cell carcinoma. Am J Epidemiol 149, 521-530

17 Brennan, J.F. et al. (1991) Acquired renal cystic disease: implications for the urologist. Br J Urol 67, 342348

18 Chow, W.H. et al. (1994) Use of analgesics and risk of renal cell cancer. Int J Cancer 59, 467-470

19 Lornoy, W. et al. (1986) Renal cell carcinoma, a new complication of analgesic nephropathy. Lancet 1 , 1271-1272

20 McCredie, M. et al. (1995) International renal-cell cancer study. II. Analgesics. Int J Cancer 60, 345-349

21 McLaughlin, J.K., Lipworth, L., Tarone, R.E., Blot, W.J. Kidney cancer, (Oxford University Press, Oxford, 2006).

22 Brauch, $H$. et al. (1999) Trichloroethylene exposure and specific somatic mutations in patients with renal cell carcinoma. J Natl Cancer Inst 91, 854-861

23 Brauch, H. et al. (2004) VHL mutations in renal cell cancer: does occupational exposure to trichloroethylene make a difference? Toxicol Lett 151, 301-310

$24 \mathrm{Yu}, \mathrm{M} . \mathrm{C}$. et al. (1986) Cigarette smoking, obesity, diuretic use, and coffee consumption as risk factors for renal cell carcinoma. J Natl Cancer Inst 77, 351-356 
25 Pelucchi, C. et al. (2002) Alcohol drinking and renal cell carcinoma in women and men. Eur J Cancer Prev 11, 543-545

26 Brownson, R.C. (1988) A case-control study of renal cell carcinoma in relation to occupation, smoking, and alcohol consumption. Arch Environ Health 43, 238-241

27 Maclure, M. and Willett, W. (1990) A case-control study of diet and risk of renal adenocarcinoma. Epidemiology 1, 430-440

28 McLaughlin, J.K. et al. (1992) Risk factors for renal-cell cancer in Shanghai, China. Int J Cancer 52, 562565

29 Benhamou, S. et al. (1993) Risk factors for renal-cell carcinoma in a French case-control study. Int J Cancer 55, 32-36

30 Kreiger, N. et al. (1993) Risk factors for renal cell carcinoma: results of a population-based case-control study. Cancer Causes Control 4, 101-110

$31 \mathrm{Hu}$, J. et al. (2003) Diet and vitamin or mineral supplements and risk of renal cell carcinoma in Canada. Cancer Causes Control 14, 705-714

32 Rashidkhani, B. et al. (2005) Major dietary patterns and risk of renal cell carcinoma in a prospective cohort of Swedish women. J Nutr 135, 1757-1762

33 Nicodemus, K.K. et al. (2004) Evaluation of dietary, medical and lifestyle risk factors for incident kidney cancer in postmenopausal women. Int J Cancer 108, 115-121

34 Mahabir, S. et al. (2005) Prospective study of alcohol drinking and renal cell cancer risk in a cohort of finnish male smokers. Cancer Epidemiol Biomarkers Prev 14, 170-175

35 Parker, A.S. et al. (2002) Gender, alcohol consumption, and renal cell carcinoma. Am J Epidemiol 155, 455-462

36 Lee, J.E. et al. (2007) Alcohol intake and renal cell cancer in a pooled analysis of 12 prospective studies. J Natl Cancer Inst 99, 801-810

37 Lamiell, J.M. et al. (1989) von Hippel-Lindau disease affecting 43 members of a single kindred. Medicine (Baltimore) 68, 1-29

38 Pavlovich, C.P. and Schmidt, L.S. (2004) Searching for the hereditary causes of renal-cell carcinoma. Nat Rev Cancer 4, 381-393

39 Washecka, R. and Hanna, M. (1991) Malignant renal tumors in tuberous sclerosis. Urology 37, 340-343

40 Breslow, N. et al. (1988) Age distribution of Wilms' tumor: report from the National Wilms' Tumor Study. Cancer Res 48, 1653-1657

41 Gallagher, D.J. et al. (2007) Wilms' tumour in adults: a case report and review of the literature. Ir J Med Sci 176, 49-51

42 Huser, J. et al. (1990) Adult Wilms' tumor: a clinicopathologic study of 11 cases. Mod Pathol 3, 321-326

43 Kovacs, G. et al. (1997) The Heidelberg classification of renal cell tumours. J Pathol 183, 131-133

44 Storkel, S. et al. (1997) Classification of renal cell carcinoma: Workgroup No. 1. Union Internationale Contre le Cancer (UICC) and the American Joint Committee on Cancer (AJCC). Cancer 80, 987-989

45 Moch, H. et al. (2000) Prognostic utility of the recently recommended histologic classification and revised TNM staging system of renal cell carcinoma: a Swiss experience with 588 tumors. Cancer 89, 604-614

46 Matsuyama, H. et al. (2005) Clinical significance of lymph node dissection in renal cell carcinoma. Scand J Urol Nephrol 39, 30-35

47 Minervini, A. et al. (2001) Regional lymph node dissection in the treatment of renal cell carcinoma: is it useful in patients with no suspected adenopathy before or during surgery? BJU Int 88, 169-172

48 Nassir, A. et al. (2002) Multilocular cystic renal cell carcinoma: a series of 12 cases and review of the literature. Urology 60, 421-427 
49 Eble, J.N. and Bonsib, S.M. (1998) Extensively cystic renal neoplasms: cystic nephroma, cystic partially differentiated nephroblastoma, multilocular cystic renal cell carcinoma, and cystic hamartoma of renal pelvis. Semin Diagn Pathol 15, 2-20

50 Corica, F.A. et al. (1999) Cystic renal cell carcinoma is cured by resection: a study of 24 cases with longterm followup. J Urol 161, 408-411

51 Gong, K. et al. (2007) Multilocular cystic renal cell carcinoma: an experience of clinical management for 31 cases. J Cancer Res Clin Oncol

52 Murad, T. et al. (1991) Multilocular cystic renal cell carcinoma. Am J Clin Pathol 95, 633-637

53 Suzigan, S. et al. (2006) Multilocular cystic renal cell carcinoma : a report of 45 cases of a kidney tumor of low malignant potential. Am J Clin Pathol 125, 217-222

54 Delahunt, B. and Eble, J.N. (1997) Papillary renal cell carcinoma: a clinicopathologic and immunohistochemical study of 105 tumors. Mod Pathol 10, 537-544

55 Lager, D.J. et al. (1995) Papillary renal tumors. Morphologic, cytochemical, and genotypic features. Cancer 76, 669-673

56 Amin, M.B. et al. (1997) Papillary (chromophil) renal cell carcinoma: histomorphologic characteristics and evaluation of conventional pathologic prognostic parameters in 62 cases. Am J Surg Pathol 21, 621635

57 Delahunt, B. et al. (2001) Morphologic typing of papillary renal cell carcinoma: comparison of growth kinetics and patient survival in 66 cases. Hum Pathol 32, 590-595

58 Crotty, T.B. et al. (1995) Chromophobe cell renal carcinoma: clinicopathological features of 50 cases. $J$ Urol 154, 964-967

59 Rumpelt, H.J. et al. (1991) Bellini duct carcinoma: further evidence for this rare variant of renal cell carcinoma. Histopathology 18, 115-122

60 Srigley, J.R. and Eble, J.N. (1998) Collecting duct carcinoma of kidney. Semin Diagn Pathol 15, 54-67

61 Swartz, M.A. et al. (2002) Renal medullary carcinoma: clinical, pathologic, immunohistochemical, and genetic analysis with pathogenetic implications. Urology 60, 1083-1089

62 Zisman, A. et al. (2002) Unclassified renal cell carcinoma: clinical features and prognostic impact of a new histological subtype. J Urol 168, 950-955

63 Argani, P. et al. (2002) PRCC-TFE3 renal carcinomas: morphologic, immunohistochemical, ultrastructural, and molecular analysis of an entity associated with the $\mathrm{t}(\mathrm{X} ; 1)(\mathrm{p} 11.2 ; \mathrm{q} 21)$. Am J Surg Pathol 26 1553-1566

64 Argani, P. et al. (2001) Primary renal neoplasms with the ASPL-TFE3 gene fusion of alveolar soft part sarcoma: a distinctive tumor entity previously included among renal cell carcinomas of children and adolescents. Am J Pathol 159, 179-192

65 Ferlicot, S. et al. (2005) A novel entity: low-grade mucinous tubular renal carcinoma. Histopathology 47, 218-219

66 Koyle, M.A. et al. (2001) Long-term urological complications in survivors younger than 15 months of advanced stage abdominal neuroblastoma. J Urol 166, 1455-1458

67 Novick AC, C.S. (2002) Renal tumors, chapter 75. 2672-2731

68 Pantuck, A.J. et al. (2001) The changing natural history of renal cell carcinoma. J Urol 166, 1611-1623

69 Patard, J.J. et al. (2005) Prognostic value of histologic subtypes in renal cell carcinoma: a multicenter experience. J Clin Oncol 23, 2763-2771

70 Smits, K.M., Schouten, L.J., van Dijk, B.A.C., Hulsbergen-van de Kaa, C.A., Wouters, K.A.D., Oosterwijk, E., van Engeland, M., vanden Brandt, P.A. (2008) Genetic and epigenetic alterations in the von HippelLindau gene: the influence on renal cancer progosis. Clin Cancer Res 14,782-787 
71 Oken, M.M. et al. (1982) Toxicity and response criteria of the Eastern Cooperative Oncology Group. Am J Clin Oncol 5, 649-655

72 Frank, I. et al. (2005) Independent validation of the 2002 American Joint Committee on cancer primary tumor classification for renal cell carcinoma using a large, single institution cohort. J Urol 173, 18891892

73 Eble JN, S.G., Epstein JI, Sesterhenn IA. (2004) WHO classification of tumours. Pathology and genetics. Tumours of the urinary system and male genital organs.

74 Fuhrman, S.A. et al. (1982) Prognostic significance of morphologic parameters in renal cell carcinoma. Am J Surg Pathol 6, 655-663

75 Kim, H.L. et al. (2003) Paraneoplastic signs and symptoms of renal cell carcinoma: implications for prognosis. J Urol 170, 1742-1746

76 Symbas, N.P. et al. (2000) Poor prognosis associated with thrombocytosis in patients with renal cell carcinoma. BJU Int 86, 203-207

77 Amin, M.B. et al. (2002) Prognostic impact of histologic subtyping of adult renal epithelial neoplasms: an experience of 405 cases. Am J Surg Pathol 26, 281-291

78 Cheville, J.C. et al. (2003) Comparisons of outcome and prognostic features among histologic subtypes of renal cell carcinoma. Am J Surg Pathol 27, 612-624

79 Sengupta, S. et al. (2005) Histologic coagulative tumor necrosis as a prognostic indicator of renal cell carcinoma aggressiveness. Cancer 104, 511-520

80 Frank, l. et al. (2002) An outcome prediction model for patients with clear cell renal cell carcinoma treated with radical nephrectomy based on tumor stage, size, grade and necrosis: the SSIGN score. J Urol 168, 2395-2400

81 Cangiano, T. et al. (1999) Sarcomatoid renal cell carcinoma: biologic behavior, prognosis, and response to combined surgical resection and immunotherapy. J Clin Oncol 17, 523-528

82 Van Poppel, H. et al. (1997) Microscopic vascular invasion is the most relevant prognosticator after radical nephrectomy for clinically nonmetastatic renal cell carcinoma. J Urol 158, 45-49

83 Eggener, S.E. et al. (2006) Renal cell carcinoma recurrence after nephrectomy for localized disease: predicting survival from time of recurrence. J Clin Oncol 24, 3101-3106

84 Elson, P.J. et al. (1988) Prognostic factors for survival in patients with recurrent or metastatic renal cell carcinoma. Cancer Res 48, 7310-7313

85 Kattan, M.W. et al. (2001) A postoperative prognostic nomogram for renal cell carcinoma. J Urol 166, 63-67

86 Lam, J.S. et al. (2005) Postoperative surveillance protocol for patients with localized and locally advanced renal cell carcinoma based on a validated prognostic nomogram and risk group stratification system. J Urol 174, 466-472; discussion 472; quiz 801

87 Leibovich, B.C. et al. (2005) A scoring algorithm to predict survival for patients with metastatic clear cell renal cell carcinoma: a stratification tool for prospective clinical trials. J Urol 174, 1759-1763; discussion 1763

88 Leibovich, B.C. et al. (2003) Scoring algorithm to predict survival after nephrectomy and immunotherapy in patients with metastatic renal cell carcinoma: a stratification tool for prospective clinical trials. Cancer 98, 2566-2575

89 Maldazys, J.D. and deKernion, J.B. (1986) Prognostic factors in metastatic renal carcinoma. J Urol 136, 376-379

90 Mekhail, T.M. et al. (2005) Validation and extension of the Memorial Sloan-Kettering prognostic factors model for survival in patients with previously untreated metastatic renal cell carcinoma. J Clin Oncol 23, $832-841$ 
91 Motzer, R.J. et al. (2002) Interferon-alfa as a comparative treatment for clinical trials of new therapies against advanced renal cell carcinoma. J Clin Oncol 20, 289-296

92 Motzer, R.J. et al. (1999) Survival and prognostic stratification of 670 patients with advanced renal cell carcinoma. J Clin Oncol 17, 2530-2540

93 Negrier, S. et al. (2002) Prognostic factors of survival and rapid progression in 782 patients with metastatic renal carcinomas treated by cytokines: a report from the Groupe Francais d'Immunotherapie. Ann Oncol 13, 1460-1468

94 Zisman, A. et al. (2001) Improved prognostication of renal cell carcinoma using an integrated staging system. J Clin Oncol 19, 1649-1657

95 Motzer, R.J. et al. (2004) Prognostic factors for survival in previously treated patients with metastatic renal cell carcinoma. J Clin Oncol 22, 454-463

96 Thompson, R.H. et al. (2004) Costimulatory B7-H1 in renal cell carcinoma patients: Indicator of tumor aggressiveness and potential therapeutic target. Proc Natl Acad Sci U S A 101, 17174-17179

97 Mizutani, Y. et al. (2005) Downregulation of Smac/DIABLO expression in renal cell carcinoma and its prognostic significance. J Clin Oncol 23, 448-454

98 Parker, A.S. et al. (2006) High expression levels of survivin protein independently predict a poor outcome for patients who undergo surgery for clear cell renal cell carcinoma. Cancer 107, 37-45

99 Velickovic, M. et al. (2002) Intragenic PTEN/MMAC1 loss of heterozygosity in conventional (clear-cell) renal cell carcinoma is associated with poor patient prognosis. Mod Pathol 15, 479-485

100 Jacobsen, J. et al. (2000) Vascular endothelial growth factor as prognostic factor in renal cell carcinoma. J Urol 163, 343-347

101 Lidgren, A. et al. (2005) The expression of hypoxia-inducible factor 1alpha is a favorable independent prognostic factor in renal cell carcinoma. Clin Cancer Res 11, 1129-1135

102 Bui, M.H. et al. (2003) Carbonic anhydrase IX is an independent predictor of survival in advanced renal clear cell carcinoma: implications for prognosis and therapy. Clin Cancer Res 9, 802-811

$103 \mathrm{Kim}$, H.L. et al. (2004) Using protein expressions to predict survival in clear cell renal carcinoma. Clin Cancer Res 10, 5464-5471

104 Gelb, A.B. et al. (1997) Appraisal of intratumoral microvessel density, MIB-1 score, DNA content, and p53 protein expression as prognostic indicators in patients with locally confined renal cell carcinoma. Cancer 80, 1768-1775

105 Leibovich, B.C. and Blute, M.L. (2006) Surgical management of renal cell carcinoma. Semin Oncol 33, 552-562

106 Flanigan, R.C. et al. (2001) Nephrectomy followed by interferon alfa-2b compared with interferon alfa2b alone for metastatic renal-cell cancer. N Engl J Med 345, 1655-1659

107 Mickisch, G.H. et al. (2001) Radical nephrectomy plus interferon-alfa-based immunotherapy compared with interferon alfa alone in metastatic renal-cell carcinoma: a randomised trial. Lancet 358, 966-970

108 McDermott, D.F. et al. (2005) Randomized phase III trial of high-dose interleukin-2 versus subcutaneous interleukin-2 and interferon in patients with metastatic renal cell carcinoma. J Clin Oncol 23, 133-141

109 Drucker, B.J. (2005) Renal cell carcinoma: current status and future prospects. Cancer Treat Rev 31 , 536-545

110 Yagoda, A. et al. (1995) Chemotherapy for advanced renal-cell carcinoma: 1983-1993. Semin Oncol 22, 42-60

111 Horio, M. et al. (1988) ATP-dependent transport of vinblastine in vesicles from human multidrugresistant cells. Proc Natl Acad Sci U S A 85, 3580-3584

112 Mignogna, C. et al. (2006) Prognostic significance of multidrug-resistance protein (MDR-1) in renal clear cell carcinomas: a five year follow-up analysis. BMC Cancer 6, 293 
113 Oliver, R.T. et al. (1989) Unexplained spontaneous regression and alpha-interferon as treatment for metastatic renal carcinoma. Br J Urol 63, 128-131

114 Childs, R. et al. (2000) Regression of metastatic renal-cell carcinoma after nonmyeloablative allogeneic peripheral-blood stem-cell transplantation. N Engl J Med 343, 750-758

115 Kubler, H. and Vieweg, J. (2006) Vaccines in renal cell carcinoma. Semin Oncol 33, 614-624

116 Yagoda, A. et al. (1993) Cytotoxic chemotherapy for advanced renal cell carcinoma. Urol Clin North Am 20, 303-321

117 Muss, H.B. et al. (1987) Recombinant alfa interferon in renal cell carcinoma: a randomized trial of two routes of administration. J Clin Oncol 5, 286-291

118 McDermott, D.F. and Atkins, M.B. (2006) Interleukin-2 therapy of metastatic renal cell carcinoma-predictors of response. Semin Oncol 33, 583-587

119 Yang, J.C. et al. (2003) Randomized study of high-dose and low-dose interleukin-2 in patients with metastatic renal cancer. J Clin Oncol 21, 3127-3132

120 Motzer, R.J. et al. (2007) Sunitinib versus interferon alfa in metastatic renal-cell carcinoma. N Engl J Med 356, 115-124

121 Escudier, B. et al. (2007) Sorafenib in advanced clear-cell renal-cell carcinoma. N Engl J Med 356, 125134

122 Hudes, G. et al. (2007) Temsirolimus, interferon alfa, or both for advanced renal-cell carcinoma. N Engl J Med 356, 2271-2281

123 Yang, J.C. et al. (2003) A randomized trial of bevacizumab, an anti-vascular endothelial growth factor antibody, for metastatic renal cancer. N Engl J Med 349, 427-434 


\section{Chapter 3}

\section{HIF signalling in renal cell carcinoma angiogenesis}

Marcella M. Baldewijns, Iris J.H. van Vlodrop, Peter B. Vermeulen, Patricia M.M.B. Soetekouw, Manon van Engeland and Adriaan P. de Bruïne

Submitted for publication 


\begin{abstract}
Hypoxia-inducible factor (HIF) plays an important role in renal tumourigenesis by transcriptional activating genes, involved in angiogenesis, glucose metabolism and tumour cell proliferation. In several renal-cancer-associated syndromes and sporadic renal cell carcinomas (RCC), HIF is already constitutively activated.

Further understanding of the molecular basis of renal carcinogenesis resulted in the development of targeted therapies, inhibiting multiple growth factor pathways, phosphatidylinositol 3-kinase-AKT-mTOR and RAS/RAF/MAP kinase pathways.

However, major challenges facing targeted therapy include selection of patients who will benefit from this therapy and obtaining clinically applicable biomarkers to monitor the activity of these agents.

In this review we discuss different aspects of angiogenesis research in RCC, including molecular background and clinical relevance.
\end{abstract}




\section{Angiogenesis in Renal Cell Carcinoma}

Renal cell carcinoma (RCC) is a heterogenous disease consisting of different histological subtypes with distinct cytogenetic abnormalities[1]. Most sporadic RCCs are clear cell renal cell carcinomas (ccRCC, 75\%) followed in frequency by papillary type I and II tumours (pRCC, 15\%) and chromophobe RCCs (chrRCC, 5\%). The high vascularity of RCC suggests that angiogenesis, the induction of a new vasculature, is integral to its pathogenesis.

Hypoxia-inducible factor (HIF) plays a central role in renal tumourigenesis by acting as a transcription factor for genes, that are involved in angiogenesis, tumour cell proliferation, cell survival and progression, metastatic spread, apoptosis and glucose metabolism[2].

In RCC, HIF can be activated by three main mechanisms. Firstly, the HIF pathway can be induced by tumour hypoxia. Tumour growth exceeding a diameter of more than 1-2 $\mathrm{mm}$ gives rise to hypoxia[3]. In a normal physiological situation, angiogenesis is regulated by a variety of activators (such as vascular endothelial growth factor (VEGF), basic-fibroblastic growth factor (bFGF) and angiopoietins) and inhibitors (such as platelet factor-4, thrombospondin-1, endostatin and angiostatin). During hypoxia, the balance is in favour of pro-angiogenesis. Angiogenic growth factors promote degradation of the extracellular matrix, endothelial cell proliferation and migration, next to structural reorganization, eventually leading to formation of new blood vessels, which is essential for tumour growth and metastasis[3].

Secondly, in several RCC-associated syndromes and sporadic RCCs, HIF is already constitutively activated. To that end, the association between HIF accumulation and Von Hippel-Lindau (VHL) gene inactivation in hereditary, and the majority of sporadic ccRCCs, is comprehensively illustrated in the literature.

Finally, oncogenic signalling contributes to HIF activation through several pathways, induced by the interaction of growth factors with their cell-surface receptors.

The highly vascularized phenotype of RCC makes it an interesting tumour for angiogenesis research, especially regarding the development and application of antiangiogenic targeted therapy.

In this review we highlight the advancements in the understanding of tumour driven angiogenesis in familial and sporadic RCC and subsequently discuss clinical implications. 


\section{Molecular basis of HIF signalling in RCC angiogenesis}

\subsection{Hypoxia induced HIF signalling}

Under normoxic conditions, HIF- $\alpha$ is hydroxylated in reactions catalyzed by both the HIF prolyl hydroxylases (PHDs) and factor inhibiting HIF (FIH). Hydroxylation by the PHDs enables $\mathrm{PVHL}$, a part of an E3 ubiquitin ligase complex to bind and ubiquitinate HIF- $\alpha$, leading to proteosomal degradation of HIF- $\alpha$ [2]. PHD activity is dependent upon oxygen and sufficient levels of 2-oxoglutarate, iron and ascorbate. Hydroxylation by FIH inhibitis HIF mediated transactivation, by blocking HIF- $\alpha$ binding to p300/CBP.

Under hypoxic conditions, HIF- $\alpha$ hydroxylation by PHDs or FIH is prevented. Unhydroxylated HIF- $\alpha$ does not bind VHL and therefore accumulates in the cell[2,5]. One of the two isoforms of HIF- $\alpha$ (HIF- $1 \alpha$ or HIF- $2 \alpha$ ) then translocates to the nucleus and dimerizes with HIF- $\beta$. The HIF- $\alpha /$ HIF- $\beta$ complex binds to hypoxia response elements (HRE) within the promoters of target genes (such as vascular endothelial growth factor (VEGF), plateled-derived growth factor (PDGF), epidermal growth factor receptor (EGFR), insulin-like growth factor (IGF), glucose transporters (GLUT-1), chemokine receptors (CXCR), and carbonic anhydrase IX (CAIX) and XII (CAXII))[2]. These genes are involved in cell growth, angiogenesis, anaerobic glucose metabolism, $\mathrm{pH}$ regulation, cell survival/apoptosis (Figure 1A).

HIF may also be activated by interaction with reactive oxygen species (ROS) probably via inhibition of PHD hydroxylation, the source of which may be from mitochondria as oxygen tension drops[4].

Hypoxia induced HIF- $\alpha$ accumulation does not only occur in RCC cells, but also in tumour-associated macrophages(TAMs) (Figure 1B). In RCC, high numbers of TAMs, preferentially present in necrotic and hypoxic areas, correlated with a poor prognosis[6]. TAMs are able to promote tumour progression by production of proangiogenic cytokines and growth factors, such as VEGF, TNF- $\alpha$, IL-8 and bFGF in response to hypoxia[7]. VEGF is a chemoattractant for macrophages, resulting in a positive feedback loop, providing a rapid vascularization of the tumour. Additionally, TAMs express angiogenesis-modulating enzymes, which contribute to the degradation of the extracellular matrix, such as matrix metalloproteinases (MMP-2, MMP-7, MMP-9, MMP-12) next to other proteins that influence angiogenesis such as urokinase-type plasminogen activator (UPA) and cyclooxygenase-2 (COX-2)[8,9]. UPA and its receptor are upregulated in TAMs in $\mathrm{RCC}[10]$. 


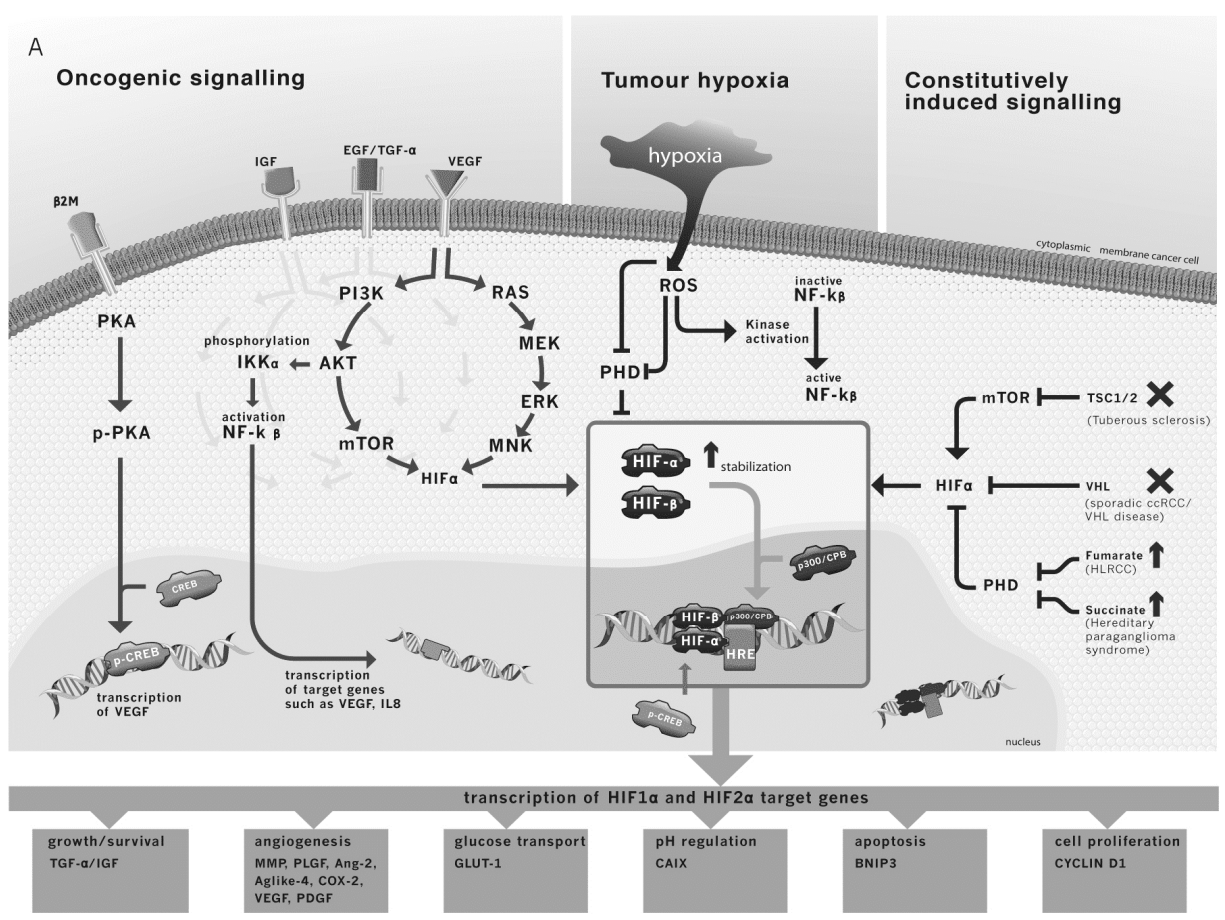

Figure 1A. In ccRCC the HIF pathway can be activated by three main mechanisms: oncogenic signalling (1), hypoxia (2) and constitutively induced signalling (3).

(1). Binding of a ligand to its receptor results in stimulation of several pathways, including the mitogenactivated protein kinase (MAPK:RAS/MNK) and phosphatidylinositol 3- OH kinase (PI3K/AKT) pathways, both promoting translation and accumulation of HIF- $\alpha$. In addition, activation of EGFR/P13K/AKT/IKK $\alpha / N F-K B$ signalling cascade, by $\mathrm{TGF} \alpha$, can induce expression of proangiogenic factors such as interleukin-8 (IL-8) and VEGF. $\beta 2$-microglobulin can activate cyclic-AMP-response-element-binding protein (CREB), through cAMPdependent Proteine Kinase A (PKA) signalling, which in turn may lead to VEGF expression. In the nucleus, HIF-1 $\alpha$ can also interact with transcription factor CREB to activate transcription.

(2). HIF degradation is dependent on hydroxylation of conserved proline residues via enzymes referred to as HIF prolyl hydroxylases (PHD). Molecular oxygen (O2) is required as a cosubstrate for PHD activity. Once hydroxylated, HIF is recognized by the Von Hippel-Lindau (VHL) protein complex and undergoes oxygendependent degradation. Tumour-associated hypoxia inhibits HIF degradation by inhibiting PHD through tricarboxylic acid cycle generation of reactive oxygen species (ROS). HIF- $\alpha$ accumulates and one of the two isoforms of HIF- $\alpha$ (HIF-1 $\alpha$ or HIF-2 $\alpha$ ) then translocates to the nucleus and dimerizes with HIF- $\beta$. The HIF$\alpha /$ HIF- $\beta$ complex binds to hypoxia response elements (HRE) within the promoters of target genes and thereby regulates transcription of genes involved in cell growth, angiogenesis, anaerobic glucose metabolism, $\mathrm{pH}$ regulation, cell survival/apoptosis, cell proliferation and other genes that modulate various cellular functions. Transcriptional co-activators are p300/CBP.

Changes in the redox potential in hypoxic cells due to the generation of ROS in mitochondria can also result in NF-KB activation.

(3). HIF- $\alpha$ can also accumulate via activation of other constitutively induced mechanisms. Loss of Tuberous sclerosis complex 1 or 2 (TSC1 or 2) function, promotes mammalian target of rapamycin (mTOR)-dependent translation and accumulation of HIF alpha in the cells.

In addition, inactivation of VHL results in stabilization and accumulation of HIF- $\alpha$. Accumulation of fumarate (as described in hereditary leiomyomatosis renal-cell cancer syndrome (HLRCC)) or accumulation of succinate (hereditary paraganglioma syndrome) causes accumulation of HIF- $\alpha$, through inhibition of PHD.

$\mathrm{TGF} \alpha=$ transforming growth factor $\alpha$; IGF = insulin-like growth factor; MMP = matrix metalloproteinase PLGF = placental growth factor; Ang-2 = angiopoietin-2; Aglike-4 = angiopoietin-like 4; COX-2 = Cyclooxygenase-2; VEGF = vascular endothelial growth factor; PDGF = plateled-derived growth factor; GLUT-1 = glucose transporter-1; CAIX = Carbonic anhydrase IX; BNIP3 $=\mathrm{BCL} 2 /$ adenovirus E1B 19kDa interacting protein 3; EGF = epidermal growth factor; NF-KB = nuclear factor-KB; IKK $\alpha=I$ kappa $B$ kinase alpha; $A K T=$ serine/threonine kinases AKT; MEK = mitogen and extracellular kinase; ERK = extracellular receptor kinase; MNK = MAPK-interacting protein kinase. For full colour picture see page 163 . 


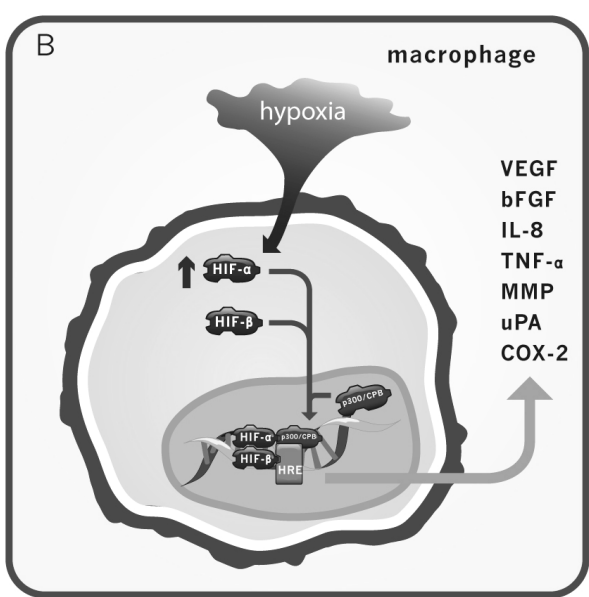

\subsection{Constitutively induced HIF signalling}

\subsubsection{Von Hippel-Lindau gene inactivation}

RCCs in patients affected by the VHL disease as well as most sporadic ccRCCs (approximately 60 to $70 \%$ ) are associated with loss of function of both alleles of the VHL gene, resulting in constitutive HIF- $1 \alpha / 2 \alpha$ activation[11].

$\mathrm{VHL}$ inactivation can be the result of mutations, deletions, loss of heterozygosity or promoter $\mathrm{CpG}$ island hypermethylation[12]. The VHL gene has been demonstrated to behave as a tumour suppressor gene[11] and its best known function is its tight regulation of HIF-1 $\alpha$ and HIF-2 $\alpha$ levels via ubiquitin-mediated proteosomal degradation of these two transcription factors by binding to the $\mathrm{pVHL}$-elongin B-elongin $\mathrm{C}$ complex[13]. The biochemical and cellular events of HIF accumulation resulting from defective VHL protein, are similar to hypoxic conditions. (Figure 1A).

HIF- $\alpha$ expression is demonstrated to be strongly upregulated in the majority of ccRCCs, in contrast to other RCC subtypes[14].

Mandriota et al. demonstrated that in the kidneys of patients with VHL disease, HIF activation is an early event occurring in morphologically normal renal tubular cells, with progressively higher protein expression in dysplastic lesions, cystic lesions and ccRCC[15]. The early loss of VHL described in this study, implicates that VHL likely serves as an "early" gatekeeper tumour suppressor gene, and it is presumed that further genetic alterations are needed for progression of preneoplastic renal cysts to RCC[15].

Especially HIF-2 $\alpha$ seems to play a crucial role with respect to VHL -/- renal carcinogenesis. First, VHL $-/-$ RCCS seem to produce both HIF-1 $\alpha$ and HIF-2 $\alpha$ or HIF-2 $\alpha$ alone[11]. Second, the appearance of HIF-2 $\alpha$ in VHL -/- preneoplastic lesions is associated with increased dysplasia[15]. Third, the elimination of HIF-2 $\alpha$ is sufficient to suppress VHL - 
HIF signalling in renal cell carcinoma

/- tumour growth in vivo[16]. Fourth, tumour suppression by $\mathrm{pVHL}$ can be overridden by HIF- $2 \alpha$ but not by HIF-1 $\alpha[16,17]$. Examination of kidneys from VHL patients likewise suggests that HIF-2 $\alpha$ is more oncogenic than HIF-1 $\alpha[15]$. This was also supported by the study of Raval et al., which showed that HIF- $1 \alpha$ and HIF- $2 \alpha$ have functionally distinct roles in the biology of VHL-defective RCC in contrast with other tumour types, with HIF-2 $\alpha$ promoting and HIF-1 $\alpha$ retarding tumour growth[17]. It was demonstrated that protumourigenic genes encoding cyclin D1, transforming growth factor- $\alpha$ and VEGF respond specifically to HIF-2 $\alpha$ and that the proapoptotic gene encoding Bnip3 responds positively to HIF- $1 \alpha$ and negatively to HIF-2 $\alpha$ [17]. These findings suggest that it is the overall balance of target gene activation that determines the outcome of HIF activation.

\subsubsection{Inactivation of fumarate hydratase gene}

Germline mutations in the fumarate hydratase (FH) tumour suppressor gene, predisposing to hereditary leiomyomatosis renal-cell cancer syndrome (HLRCC), can also alter expression levels of HIF[18]. FH encodes fumarase, an enzyme of the tricarboxylic acid (Krebs) cycle, which catalyzes the hydration of fumarate to form malate. In HLRCCs, HIF stabilization occurs via inhibition of PHDs by fumarate accumulation, due to loss of fumarase. Fumarate acts as a competitive inhibitor of the PHD cosubstrate 2oxoglutarate[18-19] (Figure 1A).

\subsubsection{Loss of tuberous sclerosis complex genes 1 and 2}

Patients with tuberous sclerosis complex develop angiomyolipomas and occasionally RCCs[20]. RCCs in tuberous sclerosis are heterogenous, including ccRCC (most commonly), pRCC and chrRCCs. [21]. Tuberous sclerosis is a genetic disorder caused by germline mutations in either the TSC1 (Tuberous sclerosis complex) gene or the TSC2 gene[20]. TSC1 and TSC2 are classical tumour suppressor genes, in which a germline mutation inactivates one allele and a somatic mutation or loss of heterozygosity (LOH) inactivates the remaining allele. Several studies have implicated the role of TSC in angiogenesis, in which the loss of TSC1/2 is accompanied by elevated levels of HIF and VEGF $[20,22,23]$. TSC2 forms a complex with TSC1, which inactivates mTOR through inhibition of Rheb activity[20]. The TSC complex can be disrupted through constitutively loss of TSC1/2 or by the growth factor-mediated PI3K/AKT signalling pathway. Enhanced activity of mTOR may explain the increased translation and activity of HIF in TSC-deficient cells (Figure 1A). In addition, Brugarolas et al. reported that TSC2 regulates VEGF not only by mTOR-dependent but also by mTOR-independent pathways. The latter pathway may involve chromatin remodelling since the HDAC inhibitor Trichostatin A downregulates VEGF in TSC2(-/-) cells[24]. 


\subsubsection{Inactivation of succinate dehydrogenase genes}

Another group of tumour suppressor mutations that shows clinical overlap with VHLrelated disease are germline mutations in genes encoding mitochondrial succinate dehydrogenase (SDHB, SDHC and SDHD), causing hereditary paraganglioma syndrome. An association between early onset RCC and mutations in SDHB is reported[25], and experimental evidence exists that this may activate HIF via an increase in succinate levels which inhibit the HIF hydroxylases[26] (Figure 1A).

\subsection{Oncogene induced HIF signalling}

HIF- $\alpha$ is also stabilized through oncogenic signalling pathways, such as phosphatidylinositol 3-OH kinase (PI3K/AKT) pathways and mitogen-activated protein kinase (MAPK:RAS/MNK) (Figure 1A). Activation of PI3K/AKT pathway promotes mTORdependent translation and accumulation of HIF- $\alpha$ [27]. Activating RAS or RAF mutations are rare in RCC, though MAPK pathway activation is observed[28].

\subsection{HIF target genes}

VEGF is a known potent mediator of angiogenesis (Figure 1C)[30]. VEGF increases vascular permeability, induces endothelial cell (EC) proliferation, survival, migration, differentiation and promotes the degradation of the extracellular matrix around sprouting ECs by inducing the expression of proteases.

VEGF-A is the prototypical member of a family of factors that also consists of VEGF-B, VEGF-C, VEGF-D and placental growth factor (PLGF), which bind differentially to VEGF receptors 1,2 and 3 and neuropilin with different specificities[29]. Alternative exon splicing results in the production of four major isoforms of human VEGF-A, VEGF121, VEGF165, VEGF189 and VEGF206 differentiated by their heparin binding affinity[30]. VEGF-A binds with high affinity to two tyrosine kinase receptors, flt-1 (VEGFR-1)and KDR (VEGFR-2)[31].

VEGF mRNA and protein are significantly elevated in RCCs compared with normal renal tissues, with some evidence to suggest a relationship between level of expression and microvessel density[30,32]. In ccRCC, tumour epithelial as well as stromal cells showed elevated VEGFR-1 mRNA levels, suggesting that VEGFR-1 signalling plays a role in renal tumourigenesis[30].

VEGFR-2 is generally believed to be the major mediator of the mitogenic, angiogenic and pro-permeability actions of VEGF-A. 


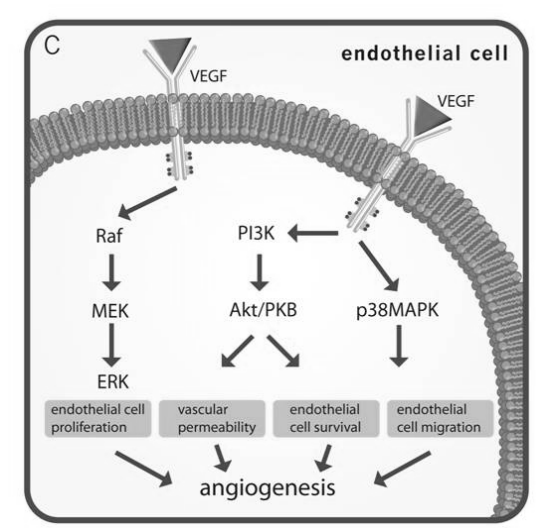

Figure 1C. Overview of the VEGF signalling transduction pathway in the endothelial cell. VEGF binding to a cognate receptor kinase (VEGFR1-2) activates the phosphatidylinositol 3-kinase (PI3K) and mitogen-activated protein kinase (MAPK) pathways. PI3K activates the downstream serine/threonine kinases AKT (also known as protein kinase $B(P K B)$ ) and mammalian target of rapamycin (mTOR).In the MAPK pathway, the extracellular signal-regulated kinase (ERK) is activated by the upstream MAP/ERK kinase (MEK). These signalling pathways result in stimulation of angiogenesis. For full colour picture see page 164.

Fox et al. demonstrated that VEGFR-2 is expressed on endothelial cells as well as RCC cells, suggesting that tumour-derived VEGF may be a powerful autocrine and paracrine growth factor in these tumours[33].

In addition to VEGF-A, VEGF-B and VEGF-C bind to tyrosine kinase receptors VEGFR-1, VEGFR-2 and flt4 (VEGFR-3). Gunningham et al. observed a significant increase in VEGF-B and its receptor VEGFR-1 but no difference in VEGF-C and its receptors between normal and renal tumour tissues [34]. These investigators also demonstrated that VEGF-B was not regulated by VHL, indicating VHL independent pathways in RCC angiogenesis.

Glucose transporter-1 (GLUT-1) facilitates the basal transport of glucose across the cellular membrane and is involved in the adaptation of the energy metabolism during hypoxia. A significant correlation was found between high GLUT-1 expression and HIF$1 \alpha$ in ccRCC[35]. Furthermore, GLUT-1 was demonstrated to be also a HIF-2 $\alpha$ target in VHL-defective RCC[36].

Carbonic anhydrase IX (CAIX), a cytosolic transmembrane protein implicated in regulating cell proliferation in response to hypoxia, is commonly overexpressed in RCC and appears to be a specific HIF-1 $\alpha$ target[36,37]. CA IX expression varies among RCC subtypes, to the extent that high levels of CAIX expression are present in ccRCC compared with minimal CA IX expression in pRCC and chrRCC[37].

Cyclooxygenase-2 (COX-2) is another key mediator of angiogenesis, and it can be induced by RAS and hypoxia. An increased expression of COX-2 in RCC is observed and a positive association between COX-2 immunostaining and vessel density was demonstrated in RCC[38].

Other angiogenesis-related growth factors demonstrated to be overexpressed in RCC are angiopoietin-like 4, plateled-derived growth factor[32], placental growth factor[32], angiopoietin-2[32,39]. 


\subsection{Hypoxia related transcription factors other than HIF}

Though most research on hypoxia/angiogenesis is focused on the HIF pathway, there are several other transcription factors that are activated by hypoxia. These include cyclic-AMP-response-element-binding protein (CREB)[40] and nuclear factor-KB (NFKB) [41].

Nomura et al. demonstrated, by use of a RCC celline, that $\beta 2$-microglobulin can activate $C R E B$, through CAMP-dependent Proteine Kinase A signalling[42]. Activation of CREB results in an increase of angiogenesis and cell proliferation via activation of VEGF signalling and its downstream survival pathways PI3K/Akt and MAPK[42].

NF-KB activation was found to stimulate angiogenesis, by inducing expression of proangiogenic factors such as interleukin-8 (IL-8) and VEGF[43]. In cCRCC biallelic inactivating mutations of VHL induce NF-KB activity through accumulation of HIF- $\alpha$ expression[44]. In turn, HIF- $\alpha$ drives expression of TGF- $\alpha$, which activates an EGFR/P13K/AKT/IKK $\alpha /$ NF-KB signalling cascade[44].

Changes in the redox potential in hypoxic cells due to the generation of ROS in mitochondria can result in NF-KB activation[41]. Additionally, during hypoxia, activation of $\mathrm{NF}-\mathrm{KB}$ can be mediated by decreased prolyl hydroxylation and subsequent degradation of IKB kinase- $\beta[45]$

\section{Assessment of angiogenesis in RCC}

\subsection{Quantification of angiogenesis}

To evaluate the implications of angiogenesis on prognosis and targeted therapy, reliable quantifying angiogenesis parameters are needed. Intra-tumoural microvessel density (MVD) is the most used quantitative parameter for angiogenesis in literature. MVD is measured by counting tumoural microvessels in a certain microscopic field, using immunohistochemical endothelial cell markers.

CCRCCs appear to be more vascularized than papillary and chromophobe RCCs[46]. We and others have shown that MVD was significantly lower in high stage (pT3-4), high grade RCCs (Fuhrman grade 3-4) than in low stage (pT1-T2), low grade RCCs (Fuhrman grade 1-2)[32,47] This finding probably reflects the inability of tumour neovascularization to keep up with tumour cell proliferation in less differentiated, more aggressive tumours. Furthermore, Delahunt et al. suggested that the vasculature of RCC is modified during tumour progression[48]. Low-grade tumours in early stages commonly have many microvessels per area, sometimes forming a syncytium of sinusoidal vessels, in contrast to high-grade, late stage RCCs, in which angiogenesis may be dependent on the development of large-diameter vascular channels, facilitating bloodborne metastatic spread. This hypothesis is supported by our morphometry study confirming the presence of fewer, but larger vessels in high grade ccRCCs[32]. 
No significant association was found between MVD and VHL alterations[49], suggesting that angiogenesis is probably influenced by a complex of paracrine and autocrine effects of growth factors, in addition to the interaction between VEGF and VHL/HIF. Moreover, Jiang et al. demonstrated that RCC cells have both VHL dependent and independent mechanisms to respond to hypoxic stress[50].

Since MVD is the net result of previous phases of tumour angiogenesis, vascular remodelling and regression, this implicates that MVD is not necessarily a reflection of ongoing tumour angiogenesis. On the contrary, EC proliferation is a better measure for active angiogenesis and this parameter might be of interest to select tumours for antiangiogenesis treatment schedules. Previously, we demonstrated a higher EC proliferation fraction in high grade versus low grade ccRCCs[32].

\subsection{Vascular differentiation}

In addition to angiogenesis quantification, the state of vascular differentiation may predict therapeutic response. CCRCCs with high MVD and low EC proliferation showed a higher mRNA expression of angiogenic growth factors, such as plateled-derived growth factor B1 and angiopoietin-1, which are related to vessel maturation and stabilization[32]. In addition, Xin Yao et al. reported two distinct types of blood vessels in cCRCC, differentiated and undifferentiated vessels, and demonstrated that a higher undifferentiated MVD significantly correlated with higher tumour grade[51] As immature vasculature has been shown to be more vulnerable for therapeutic targeting, a better response to anti-angiogenic drugs might be expected in high grade ccRCCs[52]. However, during tumour progression, selection of hypoxia resistant tumour cells can occur[53]. Nevertheless, the fact that phosphorylated VEGFR-2 is not only demonstrated in ECs but also in renal tumour cells, independent of grade, implies that antiVEGF therapy may have direct anti-tumour effects[33].

\section{Prognostic value of angiogenesis parameters}

\subsection{Angiogenic histologic parameters}

The value of MVD as a predictor of prognosis in RCC is controversial. Several reports have shown a positive correlation between MVD and survival[54,55]. Other researchers have reported an inverse relationship[48,56] or were unable to find a significant correlation between MVD and survival[57].

A study of blood vessel maturation revealed that a higher density of undifferentiated microvessels was an independent predictor of shorter survival in ccRCC[51].

\subsection{VHL status}

High pVHL expression was shown as a favourable prognostic factor in ccRCCs[58]. Studies based on genetic and epigenetic VHL alterations revealed conflicting results. While 
one study reported $\mathrm{VHL}$ alterations as a favourable independent prognostic factor for patients with stage I to III sporadic cCRCCs[59], other studies could not detect a significant correlation between VHL alterations or type of alterations and clinical outcome in sporadic ccRCC $[13,60,61]$. Clearly, to clarify the potential clinical implications of VHL alterations, additional studies should be performed and functional insight should be sought in the effect of mutations or methylation on VHL protein activity and HIF downstream pathways.

\subsection{HIF expression}

The prognostic impact of HIF-1 $\alpha$ expression has been the subject of numerous studies, often with contrasting results. Though HIF-1 $\alpha$ was reported as a favourable independent prognostic factor[62], another study showed that high HIF-1 $\alpha$ expression appeared to be an adverse prognostic factor[14]. Furthermore, some investigators reported no significant differences in survival between low and high HIF-1 $\alpha$ expression[63]. These contradictory findings may be partly due to the different methodology of HIF-1 $\alpha$ analysis. In one study only nuclear HIF-1 $\alpha$ expression was observed and analyzed[15], whereas in another study cytoplasmic HIF-1 $\alpha$ expression was evaluated[63]. This implies that the subcellular compartment (nucleus or cytoplasm) is important for the effectiveness of HIF-1 $\alpha$ as has been shown. HIF- $1 \alpha$ is indeed a transcription factor that is active when translocated to the nucleus. Higher HIF-1 $\alpha$ expression in the cytoplasm might indicate that HIF-1 $\alpha$ is retained in the cytoplasm, does not transcribe DNA and, therefore leads to less aggressive tumours and better prognosis. Di Cristofano et al. showed that a high nuclear HIF-1 $\alpha$ expression was correlated with a shorter tumourspecific survival (TSS), whereas all patients with high cytoplasmic HIF-1 $\alpha$ expression had a significantly longer TSS[64].

\subsection{HIF target genes}

VEGF and its receptors appeared to be associated with tumour stage and survival, but did not remain independent prognostic factors [65].

CAIX is highly expressed in RCC and several reports have indicated that CAIX expression appeared to be a favourable independent prognostic marker[66]. Furthermore, a higher CAIX expression was associated with a higher response to interleukin 2 (IL-2) therapy in advanced/metastatic ccRCC[67]. However, in a larger study with 730 patients with ccRCC, in which only $11 \%$ showed metastatic disease and only $6 \%$ received high-dose IL-2, CAIX did not turn out to be an independent prognostic factor[37]. Nevertheless, these contrasting findings raise the possibility that CAIX is predictive of favourable outcome for patients with metastatic disease who receive IL-2-based immunotherapy. 


\section{Targeted therapy}

Angiogenesis research contributed to the development of targeted therapy. The most significant progress has been made in the area of agents that target the VHL/HIF/VEGF pathway, which is abnormal in most patients with sporadic ccRCC.

Several agents that inhibit either the VHL/HIF/VEGF pathway or the effects of activated mTOR have recently shown considerable promise in patients with advanced renal cancer. Sunitinib and sorafenib have been found to significantly improve progression free survival in clinical trials of patients with metastatic ccRCC, who had a good or intermediate prognosis based on Memorial Sloan-Kettering cancer Center risk factor assessment (MSKCC). Therefore, these two targeted therapeutics have recently been FDA approved to treat advanced RCC[68-70]. Sunitinib is a multitargeted tyrosine kinase inhibitor of VEGFR-2, platelet-derived growth factor receptor (PDGFR), FMS-like tyrosine kinase 3 (FLT-3) and C-KIT. Sorafenib is another multikinase inhibitor targeting BRAF and CRAF, VEGFR-2, VEGFR-3, PDGFR, FLT-3 and C-KIT.

Other prominent targeted agents are bevacizumab (VEGF binding antibody) and mTOR blockers such as temsirolimus and everolimus. Bevacizumab in combination with interferon-alfa as first-line treatment in patients with $\mathrm{mRCC}$ resulted in a significant improvement in progression-free survival, compared with interferon-alfa alone[71]. Furthermore, in metastatic RCC bevacizumab is now also under investigation in combination with interleukin-2 and targeted therapies such as erlotinib, sorafenib, sunitinib and temsirolimus[72].

As compared to interferon-alpha, the mTOR inhibitor temsirolimus significantly improved overall survival in patients with metastatic RCC and poor prognosis based on MSKCC risk factor assessment[73]. Cho et al. showed that both phospho-S6 ribosomal protein and phospho-Akt expression are promising predictive biomarkers for response to temsirolimus[74]. Everolimus improves overall survival in patients with metastatic RCC who previously have been treated with a VEGF-targeted therapy[75]. These two mTOR inhibitors are still under investigation in combination with other therapies[72]. Further investigation of the molecular/pharmacodynamic effects of antiangiogenic drugs in patients is needed to improve the efficacy of these agents.

\section{Future perspectives}

Angiogenesis is a key event in RCC development and progression. With a myriad of angiogenesis inhibitors entering clinical trials for cancer treatment, stratification of patients to the likelihood of response is required. The effect of antivascular treatment is influenced by the degree of angiogenesis-(in)dependent tumour growth and the amount of ongoing angiogenesis. Both can be evaluated in nephrectomy specimens by means of suitable quantifying angiogenesis parameters. 
Furthermore, the heterogeneity in maturation of vasculature demonstrated in ccRCC[51] has therapeutic implications, since an immature vasculature is believed to be more sensitive for therapeutic targeting[52]. In addition, evaluation of angiogenesis in RCC metastases can be predictive for therapy response. Heterogeneity of angiogenic growth patterns observed among lung metastases of ccRCC[76] may influence the response to antiangiogenic therapy and can explain the resistance to specific systemic treatment modalities. Metastases with non-angiogenic growth patterns will probably not respond to antiangiogenic drugs.

Studies evaluating the prognostic relevance of angiogenic factors/parameters have often led to contrasting conclusions, due to different methodology of investigation. Until now, these variables are not used in clinical practice and the MSKCC risk factor assessment is still the most widely accepted prognostic system used for clinical trial assessment and treatment of RCC[77].

Antiangiogenic therapies can be fine-tuned based on further knowledge of the involved dominant pathway and the mechanism of action of a particular drug. Advancements in the understanding of the HIF signalling up- and downstream pathways contributed to the development of effective targeted therapy. Nevertheless, the fact that angiogenesis parameters are not significantly associated with VHL alterations, which is one of the key events in the HIF signalling pathway in CCRCC, suggests that there is a more complex interplay of additional HIF downstream modifications, which still have to be elucidated. In addition to genetic changes, insight into the functional significance of epigenetic changes in the HIF downstream pathway may contribute to the development of new therapeutic approaches by use of demethylating agents.

Since current antiangiogenic therapies are evidencing target-related toxicities, there is an urgent need to develop and validate novel surrogate biomarkers for defining optimal drug doses and schedules, monitoring drug efficiency, and predicting drug response.

Microarrays to study mRNA expression patterns will be helpful in identifying groups of genes predictive of response to anti-angiogenic treatment[78,79].

Current markers such as circulating plasma proteins, circulating endothelial cells and imaging of tumour vessels themselves or the effect of therapy on the tumour cells (Fluorodeoxyglucose positron emission tomography) are under investigation[80]. 


\section{References}

1 Pavlovich, C.P. and Schmidt, L.S. (2004) Searching for the hereditary causes of renal-cell carcinoma. Nat Rev Cancer 4, 381-393

2 Semenza, G.L. (2003) Targeting HIF-1 for cancer therapy. Nat Rev Cancer 3, 721-732

3 Naumov, G.N. et al. (2006) Role of angiogenesis in human tumor dormancy: animal models of the angiogenic switch. Cell Cycle 5, 1779-1787

4 Dewhirst, M.W. et al. (2008) Cycling hypoxia and free radicals regulate angiogenesis and radiotherapy response. Nat Rev Cancer 8, 425-437

5 Pugh, C.W. and Ratcliffe, P.J. (2003) The von Hippel-Lindau tumor suppressor, hypoxia-inducible factor1 (HIF-1) degradation, and cancer pathogenesis. Semin Cancer Biol 13, 83-89

6 Hamada, I. et al. (2002) Clinical effects of tumor-associated macrophages and dendritic cells on renal cell carcinoma. Anticancer Res 22, 4281-4284

7 Pollard, J.W. (2004) Tumour-educated macrophages promote tumour progression and metastasis. Nat Rev Cancer 4, 71-78

8 Lewis, C.E. and Pollard, J.W. (2006) Distinct role of macrophages in different tumor microenvironments. Cancer Res 66, 605-612

9 Hemmerlein, B. et al. (2004) Comparative analysis of COX-2, vascular endothelial growth factor and microvessel density in human renal cell carcinomas. Histopathology 45, 603-611

10 Ohba, K. et al. (2005) Expression of urokinase-type plasminogen activator, urokinase-type plasminogen activator receptor and plasminogen activator inhibitors in patients with renal cell carcinoma: correlation with tumor associated macrophage and prognosis. $J$ Urol 174, 461-465

11 Kaelin, W.G., Jr. (2007) The von Hippel-Lindau tumor suppressor protein and clear cell renal carcinoma. Clin Cancer Res 13, 680s-684s

12 Banks, R.E. et al. (2006) Genetic and epigenetic analysis of von Hippel-Lindau (VHL) gene alterations and relationship with clinical variables in sporadic renal cancer. Cancer Res 66, 2000-2011

13 Kim, W.Y. and Kaelin, W.G. (2004) Role of VHL gene mutation in human cancer. J Clin Oncol 22, 49915004

14 Klatte, T. et al. (2007) Hypoxia-Inducible Factor 1 \{alpha\} in Clear Cell Renal Cell Carcinoma. Clin Cancer Res 13, 7388-7393

15 Mandriota, S.J. et al. (2002) HIF activation identifies early lesions in VHL kidneys: evidence for sitespecific tumor suppressor function in the nephron. Cancer Cell 1, 459-468

16 Kondo, K. et al. (2003) Inhibition of HIF2alpha is sufficient to suppress pVHL-defective tumor growth. PLOS Biol 1, E83

17 Raval, R.R. et al. (2005) Contrasting properties of hypoxia-inducible factor 1 (HIF-1) and HIF-2 in von Hippel-Lindau-associated renal cell carcinoma. Mol Cell Biol 25, 5675-5686

18 Isaacs, J.S. et al. (2005) HIF overexpression correlates with biallelic loss of fumarate hydratase in renal cancer: novel role of fumarate in regulation of HIF stability. Cancer Cell 8, 143-153

19 Pollard, P.J. et al. (2007) Targeted inactivation of Fh1 causes proliferative renal cyst development and activation of the hypoxia pathway. Cancer Cell 11,311-319

20 Mak, B.C. and Yeung, R.S. (2004) The tuberous sclerosis complex genes in tumor development. Cancer Invest 22, 588-603

21 Jimenez, R.E. et al. (2001) Concurrent angiomyolipoma and renal cell neoplasia: a study of 36 cases. Mod Pathol 14, 157-163

22 El-Hashemite, N. et al. (2003) Loss of Tsc1 or Tsc2 induces vascular endothelial growth factor production through mammalian target of rapamycin. Cancer Res 63, 5173-5177 
23 Liu, M.Y. et al. (2003) Up-regulation of hypoxia-inducible factor 2alpha in renal cell carcinoma associated with loss of Tsc- 2 tumor suppressor gene. Cancer Res 63, 2675-2680

24 Brugarolas, J.B. et al. (2003) TSC2 regulates VEGF through mTOR-dependent and -independent pathways. Cancer Cell 4, 147-158

25 Vanharanta, S. et al. (2004) Early-onset renal cell carcinoma as a novel extraparaganglial component of SDHB-associated heritable paraganglioma. Am J Hum Genet 74, 153-159

26 Selak, M.A. et al. (2005) Succinate links TCA cycle dysfunction to oncogenesis by inhibiting HIF-alpha prolyl hydroxylase. Cancer Cell 7, 77-85

27 Hay, N. (2005) The Akt-mTOR tango and its relevance to cancer. Cancer Cell 8, 179-183

28 Rochlitz, C.F. et al. (1992) Mutations in the ras protooncogenes are rare events in renal cell cancer. Eur J Cancer 28, 333-336

29 Ferrara, N. et al. (2003) The biology of VEGF and its receptors. Nat Med 9, 669-676

30 Rivet, J. et al. (2008) VEGF and VEGFR-1 are coexpressed by epithelial and stromal cells of renal cell carcinoma. Cancer 112, 433-442

31 Roskoski, R., Jr. (2008) VEGF receptor protein-tyrosine kinases: structure and regulation. Biochem Biophys Res Commun 375, 287-291

32 Baldewijns, M.M. et al. (2007) High-grade clear cell renal cell carcinoma has a higher angiogenic activity than low-grade renal cell carcinoma based on histomorphological quantification and qRT-PCR mRNA expression profile. Br J Cancer 96, 1888-1895

33 Fox, S.B. et al. (2004) Phosphorylated KDR is expressed in the neoplastic and stromal elements of human renal tumours and shuttles from cell membrane to nucleus. J Pathol 202, 313-320

34 Gunningham, S.P. et al. (2001) Vascular endothelial growth factor-B and vascular endothelial growth factor-C expression in renal cell carcinomas: regulation by the von Hippel-Lindau gene and hypoxia. Cancer Res 61, 3206-3211

35 Lidgren, A. et al. (2008) Glucose transporter-1 expression in renal cell carcinoma and its correlation with hypoxia inducible factor-1 alpha. BJU Int 101, 480-484

$36 \mathrm{Hu}, \mathrm{C.J}$. et al. (2003) Differential roles of hypoxia-inducible factor 1alpha (HIF-1alpha) and HIF-2alpha in hypoxic gene regulation. Mol Cell Biol 23, 9361-9374

37 Leibovich, B.C. et al. (2007) Carbonic anhydrase IX is not an independent predictor of outcome for patients with clear cell renal cell carcinoma. J Clin Oncol 25, 4757-4764

38 Miyata, Y. et al. (2003) Expression of cyclooxygenase-2 in renal cell carcinoma: correlation with tumor cell proliferation, apoptosis, angiogenesis, expression of matrix metalloproteinase-2, and survival. Clin Cancer Res 9, 1741-1749

39 Le Jan, S. et al. (2003) Angiopoietin-like 4 is a proangiogenic factor produced during ischemia and in conventional renal cell carcinoma. Am J Pathol 162, 1521-1528

40 Taylor, C.T. et al. (2000) Phosphorylation-dependent targeting of cAMP response element binding protein to the ubiquitin/proteasome pathway in hypoxia. Proc Natl Acad Sci U S A 97, 12091-12096

41 Lluis, J.M. et al. (2007) Dual role of mitochondrial reactive oxygen species in hypoxia signaling: activation of nuclear factor-\{kappa\}B via c-SRC and oxidant-dependent cell death. Cancer Res 67, 7368-7377

42 Nomura, T. et al. (2006) Beta2-microglobulin promotes the growth of human renal cell carcinoma through the activation of the protein kinase A, cyclic AMP-responsive element-binding protein, and vascular endothelial growth factor axis. Clin Cancer Res 12, 7294-7305

43 Karin, M. et al. (2002) NF-kappaB in cancer: from innocent bystander to major culprit. Nat Rev Cancer 2 301-310

44 An, J. and Rettig, M.B. (2005) Mechanism of von Hippel-Lindau protein-mediated suppression of nuclear factor kappa B activity. Mol Cell Biol 25, 7546-7556 
45 Cummins, E.P. et al. (2006) Prolyl hydroxylase-1 negatively regulates IkappaB kinase-beta, giving insight into hypoxia-induced NFkappaB activity. Proc Natl Acad Sci U S A 103, 18154-18159

46 Brieger, J. et al. (1999) Inverse regulation of vascular endothelial growth factor and VHL tumor suppressor gene in sporadic renal cell carcinomas is correlated with vascular growth: an in vivo study on 29 tumors. J Mol Med 77, 505-510

47 Yildiz, E. et al. (2008) Relation of microvessel density with microvascular invasion, metastasis and prognosis in renal cell carcinoma. BJU Int 101, 758-764

48 Delahunt, B. et al. (1997) Prognostic significance of microscopic vascularity for clear cell renal cell carcinoma. Br J Urol 80, 401-404

49 Schraml, P. et al. (2002) VHL mutations and their correlation with tumour cell proliferation, microvessel density, and patient prognosis in clear cell renal cell carcinoma. J Pathol 196, 186-193

50 Jiang, Y. et al. (2003) Gene expression profiling in a renal cell carcinoma cell line: dissecting VHL and hypoxia-dependent pathways. Mol Cancer Res 1, 453-462

51 Yao, X. et al. (2007) Two distinct types of blood vessels in clear cell renal cell carcinoma have contrasting prognostic implications. Clin Cancer Res 13, 161-169

52 Feron, O. (2004) Targeting the tumor vascular compartment to improve conventional cancer therapy. Trends Pharmacol Sci 25, 536-542

$53 \mathrm{Yu}$, J.L. et al. (2002) Effect of p53 status on tumor response to antiangiogenic therapy. Science 295, 1526-1528

54 Sabo, E. et al. (2001) Microscopic analysis and significance of vascular architectural complexity in renal cell carcinoma. Clin Cancer Res 7, 533-537

55 Rioux-Leclercq, N. et al. (2001) Clinical significance of cell proliferation, microvessel density, and CD44 adhesion molecule expression in renal cell carcinoma. Hum Pathol 32, 1209-1215

56 Joo, H.J. et al. (2004) Increased expression of caveolin-1 and microvessel density correlates with metastasis and poor prognosis in clear cell renal cell carcinoma. BJU Int 93, 291-296

57 Minardi, D. et al. (2005) Prognostic role of Fuhrman grade and vascular endothelial growth factor in pT1a clear cell carcinoma in partial nephrectomy specimens. J Urol 174, 1208-1212

58 Parker, A.S. et al. (2005) Loss of expression of von Hippel-Lindau tumor suppressor protein associated with improved survival in patients with early-stage clear cell renal cell carcinoma. Urology 65, 10901095

59 Yao, M. et al. (2002) VHL tumor suppressor gene alterations associated with good prognosis in sporadic clear-cell renal carcinoma. J Nat/ Cancer Inst 94, 1569-1575

$60 \mathrm{Kim}$, J.H. et al. (2005) Somatic VHL alteration and its impact on prognosis in patients with clear cell renal cell carcinoma. Oncol Rep 13, 859-864

61 Smits, K.M. et al. (2008) Genetic and Epigenetic Alterations in the von Hippel-Lindau Gene: the Influence on Renal Cancer Prognosis. Clin Cancer Res 14, 782-787

62 Lidgren, A. et al. (2005) The expression of hypoxia-inducible factor 1alpha is a favorable independent prognostic factor in renal cell carcinoma. Clin Cancer Res 11, 1129-1135

63 Lidgren, A. et al. (2006) Hypoxia-inducible factor 1alpha expression in renal cell carcinoma analyzed by tissue microarray. Eur Urol 50, 1272-1277

64 Di Cristofano, C. et al. (2007) Nuclear expression of hypoxia-inducible factor-1alpha in clear cell renal cell carcinoma is involved in tumor progression. Am J Surg Pathol 31, 1875-1881

65 Ljungberg, B.J. et al. (2006) Different vascular endothelial growth factor (VEGF), VEGF-receptor 1 and -2 mRNA expression profiles between clear cell and papillary renal cell carcinoma. BJU Int 98, 661-667

66 Bui, M.H. et al. (2003) Carbonic anhydrase IX is an independent predictor of survival in advanced renal clear cell carcinoma: implications for prognosis and therapy. Clin Cancer Res 9, 802-811 
67 Atkins, M. et al. (2005) Carbonic anhydrase IX expression predicts outcome of interleukin 2 therapy for renal cancer. Clin Cancer Res 11, 3714-3721

68 Rock, E.P. et al. (2007) Food and Drug Administration drug approval summary: Sunitinib malate for the treatment of gastrointestinal stromal tumor and advanced renal cell carcinoma. Oncologist 12, 107-113

69 Motzer, R.J. et al. (2007) Sunitinib versus interferon alfa in metastatic renal-cell carcinoma. N Engl J Med 356, 115-124

70 Escudier, B. et al. (2007) Sorafenib in advanced clear-cell renal-cell carcinoma. N Engl J Med 356, 125134

71 Escudier, B. et al. (2007) Bevacizumab plus interferon alfa-2a for treatment of metastatic renal cell carcinoma: a randomised, double-blind phase III trial. Lancet 370, 2103-2111

72 Atkins, M.B. et al. (2007) Innovations and challenges in renal cell carcinoma: summary statement from the Second Cambridge Conference. Clin Cancer Res 13, 667s-670s

73 Hudes, G. et al. (2007) Temsirolimus, interferon alfa, or both for advanced renal-cell carcinoma. N Engl J Med 356, 2271-2281

74 Cho, D. et al. (2007) Potential histologic and molecular predictors of response to temsirolimus in patients with advanced renal cell carcinoma. Clin Genitourin Cancer 5, 379-385

75 Motzer, R.J. et al. (2008) Efficacy of everolimus in advanced renal cell carcinoma: a double-blind, randomised, placebo-controlled phase III trial. Lancet 372, 449-456

76 Sardari Nia, P. et al. (2007) Distinct angiogenic and non-angiogenic growth patterns of lung metastases from renal cell carcinoma. Histopathology 51, 354-361

77 Motzer, R.J. et al. (1999) Survival and prognostic stratification of 670 patients with advanced renal cell carcinoma. J Clin Oncol 17, 2530-2540

78 Lam, J.S. et al. (2004) Tissue array-based predictions of pathobiology, prognosis, and response to treatment for renal cell carcinoma therapy. Clin Cancer Res 10, 6304S-6309S

79 Tan, M.H. et al. (2004) Gene expression profiling of renal cell carcinoma. Clin Cancer Res 10, 6315S$6321 \mathrm{~S}$

80 Bhatt, R.S. et al. (2007) Biomarkers for monitoring antiangiogenic therapy. Clin Cancer Res 13, 777s 780 s 


\section{Chapter 4}

High grade clear cell renal cell carcinoma has a higher angiogenic activity than low grade renal cell carcinoma based on histomorphological quantification and qRT-PCR mRNA expression profile.

MM Baldewijns, VL Thijssen, GG Van den Eynden, SJ Van Laere, AM Bluekens, T Roskams, $\mathrm{H}$ van Poppel, AP De Bruïne, AW Griffioen and PB Vermeulen

Br J Cancer 2007;96(12):1888-95. 


\begin{abstract}
Clear cell renal cell carcinoma (ccRCC) is a highly vascularised tumour and is therefore an attractive disease to study angiogenesis and to test novel angiogenesis inhibitors in early clinical development. Endothelial cell proliferation plays a pivotal role in the process of angiogenesis.

The aim of this study was to compare angiogenesis parameters in low nuclear grade $(n=87)$ versus high nuclear grade ccRCC $(n=63)$. A panel of antibodies was used for immunohistochemistry: CD34/Ki-67, carbonic anhydrase IX (CAIX), hypoxia-inducible factor-1 $\alpha$ (HIF-1 $\alpha)$ and vascular endothelial growth factor (VEGF). Vessel density (MVD), endothelial cell proliferation fraction (ECP\%) and tumour cell proliferation fraction (TCP\%) were assessed. mRNA expression levels of angiogenesis stimulators and inhibitors were determined by quantitative RT-PCR.

High grade ccRCC showed a higher ECP\% $(p=0.049)$, a higher TCP\% $(p=0.009)$, a higher VEGF protein expression $(p<0.001)$, a lower MVD $(p<0.001)$ and a lower HIF$1 \alpha$ protein expression $(p=0.002)$ than low grade ccRCC. Growth factor mRNA expression analyses revealed a higher expression of ANG2 in low grade CCRCC. MVD and ECP\% were inversely correlated ( $R h o=-0.26, p=0.001$ ). Because of the imperfect association of nuclear grade and ECP\% or MVD, CCRCC were also grouped based on low/high MVD and ECP\%. This analysis revealed a higher expression of vessel maturation and stabilization factors (PLGF, PDGFB1, ANG1) in CCRCC with high MVD, a group of ccRCC highly enriched in low nuclear grade ccRCC, with low ECP\%.

Our results suggest heterogeneity in angiogenic activity and vessel maturation of cCRCC, to a large extent linked to nuclear grade, and, with probable therapeutic implications.
\end{abstract}




\section{Introduction}

Clear cell renal cell carcinoma (ccRCC) is the most common carcinoma of the renal tubular epithelium accounting for approximately $75 \%$ of cases in surgical series [1]. Aberrations of the von Hippel-Lindau tumour suppressor gene (VHL) have been shown to be an early and distinct event in the development of cCRCC [2]. In up to $70 \%$ of ccRCC, somatic $V H L$ gene inactivation occurs [3]. The $V H L$ gene product ( $p V H L$ ) binds the hypoxia-inducible factor $1 \alpha$ (HIF-1 $\alpha$ ) in normoxic cells. Lack of VHL protein leads to stabilization of HIF-1 $\alpha$, a state which is normally seen only in hypoxic cells. The increased levels of HIF-1 $\alpha$ in ccRCC [4] are thus mainly caused by genetic alterations of the $V H L$ gene in addition to or despite stimulation through hypoxia. Stabilization of HIF- $1 \alpha$ activates a cascade of pathways which include angiogenesis, glycolysis, proliferation and alterations in microenvironmental pH ( carbonic anhydrase IX (CA IX)) [5]. Overexpression of angiogenic factors due to HIF- $1 \alpha$ upregulation can explain the hypervascular nature of ccRCC.

Tumour angiogenesis is a complex process that is regulated by a balance between proangiogenic and anti-angiogenic factors. This balance is influenced by tumour cells and the tumour's micro-environment [6,7]. The main player in angiogenesis is the endothelial cell (EC), and numerous activators of EC proliferation and migration have been described. Most of these activators are receptor kinase ligands, such as vascular endothelial growth factor (VEGF), fibroblast growth factor (FGF), plateled-derived growth factor (PDGF) and epidermal growth factor (EGF), but they can also be of different origin, such as lysophosphatic acid (LPA) or interleukin-8 (IL8), tumour necrosis factor$\alpha$ (TNF- $\alpha$ ) [8-10]. Natural occurring angiogenic inhibitors include statins (e.g. endostatin, angiostatin), Thrombospondin 1 (TSP1) and plateled derived factor 4 (PF4). ccRCC has an inpredictable course. The most widely used tumour-related prognostic factors in RCC include stage, Fuhrman nuclear grade and histological type [11]. Additional markers, still under investigation, are cellular proliferation, apoptosis and angiogenesis, among others.

Since the highly vascularized phenotype of ccRCC suggests that angiogenesis is integral to its pathogenesis, the aim of our study was to compare the angiogenic potential of low grade ccRCC (Fuhrman grade1-2) with high grade ccRCC (Fuhrman grade 3-4). To that end, EC proliferation, a measure of ongoing angiogenesis, was immunohistochemically assessed and correlated with vessel density (MVD), tumour cell proliferation, HIF-1 $\alpha$, VEGF and CAIX expression. Additionally, the mRNA expression levels of $\operatorname{VEGF}(-A / B / C)$, bFGF, placental growth factor (PLGF), Angiopoietin 1 (ANG1), angiopoietin 2 (ANG2), PDGFB1, EGF, TNF- $\alpha$, transforming growth factor- $\alpha$ (TGF- $\alpha$ ), transforming growth factor- $\beta$ (TGF- $\beta$ ), IL8, PF4, TSP1, vascular endothelial receptors 1 and 2 (VEGFR1 andVEGFR2) were determined within low and high grade CCRCC and correlated with immunohistochemical angiogenesis data. 


\section{Material and methods}

\subsection{Patients and tissue specimens}

Tumour samples of 150 patients with ccRCC, treated with radical or partial nephrectomy were collected retrospectively. The samples were derived from the archives of the Departments of Histopathology of the University Hospitals of Leuven and Maastricht. None of the patients received any cancer-related therapy prior to surgery. The original histological slides, stained with hematoxylin and eosin, were reviewed to confirm nuclear Fuhrman grading. All patients were evaluated post-operatively at regular intervals by means of physical examination, chest x-ray, abdominal computed tomography or ultrasound (median follow-up 64 months, range 1-153 months). When indicated, a bone scan was performed. The clinico-pathological data of the study population are summarized in Table 1.

Table 1: Clinical and histopathological characteristics

\begin{tabular}{ll}
\hline & Clinico-pathological data $\mathbf{~} \mathbf{N}=\mathbf{1 5 0})$ \\
\hline Gender $\mathrm{m} / \mathrm{f}$ & $99 / 51(66 / 34 \%)$ \\
Age (years) & $66.6(12.1 \mathrm{SD})$ \\
Mean tumour diameter & $6.36( \pm 3.45 \mathrm{SD})$ \\
Median tumour diameter & $6.00(0.7-20.0$ range $)$ \\
Fuhrman grade & $15(10.0 \%)$ \\
1 & $72(48.0 \%)$ \\
2 & $46(30.7 \%)$ \\
3 & $17(11.3 \%)$ \\
4 & \\
Tumour status ${ }^{a}$ & $82(54.7 \%)$ \\
T1 & $24(16.0 \%)$ \\
T2 & $42(28.0)$ \\
T3 & $2(1.3 \%)$ \\
T4 & \\
Lymph node status & \\
1 & 4 \\
2 & 11 \\
\hline a ${ }^{a}$ and N status were assigned according to the TNM classification of the International Union Against \\
cancer 2002
\end{tabular}

\subsection{Quantification of microvessel density (MVD), endothelial cell proliferation fraction (ECP\%) and tumour cell proliferation fraction (TCP\%)}

Serial sections of 4 micron were made from the paraffin embedded kidney tumours. A CD34/Ki67 double-stain procedure was performed on an automated immunohistochemical (IHC) staining system (Dako Autostainer; Dako, Glostrüp, Denmark), as described before [12]. This technique is used to simultaneously stain endothelial cells (cytoplasmatic CD34) and proliferating cells (nuclear Ki-67). Anti-Ki-67 (clone MIB-1, dilution 1/150, Dako, Glöstrup, Denmark) antibody binding was visualised with secondary antibody labelled polymers containing peroxidase with $D A B$ as a substrate. For detection of anti-CD34 (clone QBEnd/10, dilution 1/50, Dako) binding, secondary anti- 
body labelled with an alkaline group and fast red substrate were used. One section was analysed per tumour. For assessment of the vascular density within a tumour section, 1 hotspot (most vascularized microscopic field) was selected and 4 areas were chosen randomly. Vessel counts were done at 200x magnification using an optical grid. The presence of a vascular lumen was not necessary to identify a microvessel. MVD was expressed as vessels per $\mathrm{mm}^{2}$. Next, a total number of approximately 500 intratumoural endothelial cells and 500 tumour cells were evaluated on consecutive fields at a 400x magnification and the fractions of proliferating endothelial cells and tumour cells were assessed. ECP\% and TCP\% were calculated according to the following formulas: $\mathrm{ECP} \%=$ (the number of endothelial cells with Ki67-stained nuclei/total number of endothelial cells evaluated) $\times 100 ; \mathrm{TCP} \%=$ (the number of tumour cells with Ki67stained nuclei/total number of tumour cells evaluated) $\times 100$. Furthermore, in a subgroup of 15 grade 1 and 15 grade 4 ccRCC vessel area was determined by means of Leica Qwin morphometry system (Version 3.2.1, Leica Cambridge, UK).

\subsection{Assesment of HIF-1a, CA IX and VEGF}

CAIX IHC staining was automated using the Dako Autostainer (Dako). Primary antibody anti-CAIX (Rabbit polyclonal, diluted 1/2000, Novus Biologicals, Littleton, CO, USA) was incubated for $60 \mathrm{~min}$ at room temperature. Antibody binding was visualized with the ChemMate Envision+detection system (Dako). Semiquantitative analysis of CAIX expression was performed as described before [12-14]. In short, a score of 0-3 for intensity of staining was given (0:no staining, 1:weak staining, 2:moderate staining, 3:strong staining). The percentage of immunostained tumour cells was estimated. The product (intensity score $x$ the percentage of immunoreactive tumour cells) yielded a final score of 0-300.

The HIF-1 $\alpha$ IHC staining was carried out manually, as described elsewhere [12], using an anti-HIF-1 $\alpha$ primary antibody (Clone 54, diluted 1/500, BD Pharmigen, Franklin Lake, NJ, USA). For HIF-1 $\alpha$ expression analysis, only cells with completely and darkly stained nuclei were scored as positive. The fraction of HIF-1 $\alpha$-positive tumour cells was estimated.

The VEGF IHC staining was performed with Rabbit polyclonal antisera to VEGF reacting with VEGF A isoforms 165 and 121 (Peprotech Inc, Rocky Hill, New York) at a 1/50 dilution. After rinsing with TBS, the slides were blocked for aspecific antibody binding with $1 \%$ normal goat serum in TBS for 30 minutes, followed by incubation with the primary rabbit anti-human VEGF-A antibody. After washing with TBS the slides were incubated with Powervision poly HRP goat anti-mouse/rabbit/rat (Immunologic/Klinpath,NL,DPVO-55hrp) for 30 minutes. Subsequently, an incubation was performed with avidine-biotin complex HRP (Dako) and developed with diaminobenzidine (DAB, Sigma, Zwijndrecht, The Netherlands) for 10 minutes. The slides were counterstained with haematoxylin (Merck, Darmst, Germany) and mounted for lightmicroscopical evaluation. As negative control we used slides with normal kidney and 
RCC tissue, without application of the primary antibody. The staining was semiquantitatively assessed according to a four point grading scale:

0 absence of tumour staining, $1+$ membrane staining tumour cells, $2+$ strong membrane staining and cytoplasmatic staining of $<50 \%$ of the tumour cells and $3+$ strong cytoplasmic staining in $>50 \%$ of all tumour cells.

\subsection{RNA isolation, cDNA synthesis and qPCR analysis}

From 33 frozen tumour tissues and 16 normal renal tissues, total RNA was isolated from 10 cryosections (20 micron) with the RNeasy mini kit (QIAgen) according the supplier's protocol. Possible genomic DNA contamination was removed by on column DNasel treatment for 20 minutes at room temperature. Concentration and quality of the RNA was analysed on the NanoDrop ND-1000 (Nanodrop Technologies Inc.) and by agarose gel electrophoresis, respectively. A total amount of $100 \mathrm{ng}$ total RNA was used for cDNA synthesis with the iscript cDNA synthesis kit (Biorad) according the supplier's protocol. Quantitative PCR was performed with the iCycler (Biorad) in a total volume of $25 \mathrm{uL}$ on $30 \mathrm{ng}$ CDNA with the iQ SYBR Green Supermix (Biorad) and $400 \mathrm{nM}$ forward and reverse primer. The primers used for this study have been described before or were designed and validated as described previously [15]. Primers were synthesized by Eurogentec and targeted against CyclophilinA, beta-actin, 18S rRNA, VEGF (VEGF-A), VEGF-B, VEGF-C, bFGF, PLGF, ANG1, ANG2, EGF, PDGFB1, TNF- $\alpha$, IL8, PF4, TGF- $\alpha$, TGF$\beta$, TSP-1, VEGFR1, and VEGFR2.

\section{Statistical analysis}

Statistical analysis was performed using the SPSS 12.0 software package. A P-value $\leq$ 0.05 was statistically significant, a $0.05<\mathrm{P}$-value $\leq 0.1$ was considered a trend towards statistical significance. Normality was tested with a Kolmogorov-Smirnov test assuming normality of data if $P \geq 0.2$. If continuous data were normally distributed, correlations were analysed with Pearson's correlation statistics, if not, with Spearman's correlation statistics. In case of normal distribution in all subgroups, equality of means was tested with a student T-test. If data were not normally distributed, equality of medians was tested with a Mann-Whitney U-test. For analyzing correlations between categorical variables (e.g. high/low ecp\%, high/low mvd, high/low grade), a $\chi^{2}$ test or, when the assumptions for the $\chi^{2}$ test were not met, the Fisher's exact test was used. 


\section{Results}

\subsection{Angiogenesis in low grade cCRCC compared with high grade cCRCC}

Median MVD was 181.1 (range 27.6 - 481.8), median ECP\% was 0.7\% (range 0 - 13.2\%) and median TCP\% was $3.8 \%$ (range $0-46.0 \%$ ). CAIX and HIF-1 $\alpha$ were expressed in respectively $97 \%$ and $88 \%$ of all tumours. VEGF immunostaining was present in all ccRCC and was observed mainly in tumour cells and in endothelial cels of intratumoural vessels (figure 1).

VEGF immunostaining was graded 1+ in 59 tumours (39.3\%), 2+ in 66 tumours (44\%) and $3+$ in 25 tumours (16.7\%). VEGF protein expression was inversely related to MVD (Rho=-0.42, $\mathrm{p}<0.001$ ). There was an inverse correlation between MVD and Fuhrman grade (Rho=-0.40, $\mathrm{p}<0.001$ ). ECP\% had a moderate positive correlation with Fuhrman grade (Rho=0.17, $\mathrm{p}=0.029$ ). $\mathrm{MVD}$ and $\mathrm{ECP} \%$ were inversely correlated (Rho=-0.26, $\mathrm{p}=0.001)$.

Based on these results, the group of ccRCC was split in 2 groups: high grade (Fuhrman grade 3 and 4) and low grade (Fuhrman grade 1 and 2). Table 2 shows the differences in angiogenesis parameters and hypoxia-related parameters between both subpopulations. High grade CCRCC had higher ECP\% (Figure 2), higher TCP\%, larger vessel area and higher VEGF protein expression. In contrast, MVD, CAIX protein expression and HIF-1 $\alpha$ protein expression were lower in high grade tumours. 

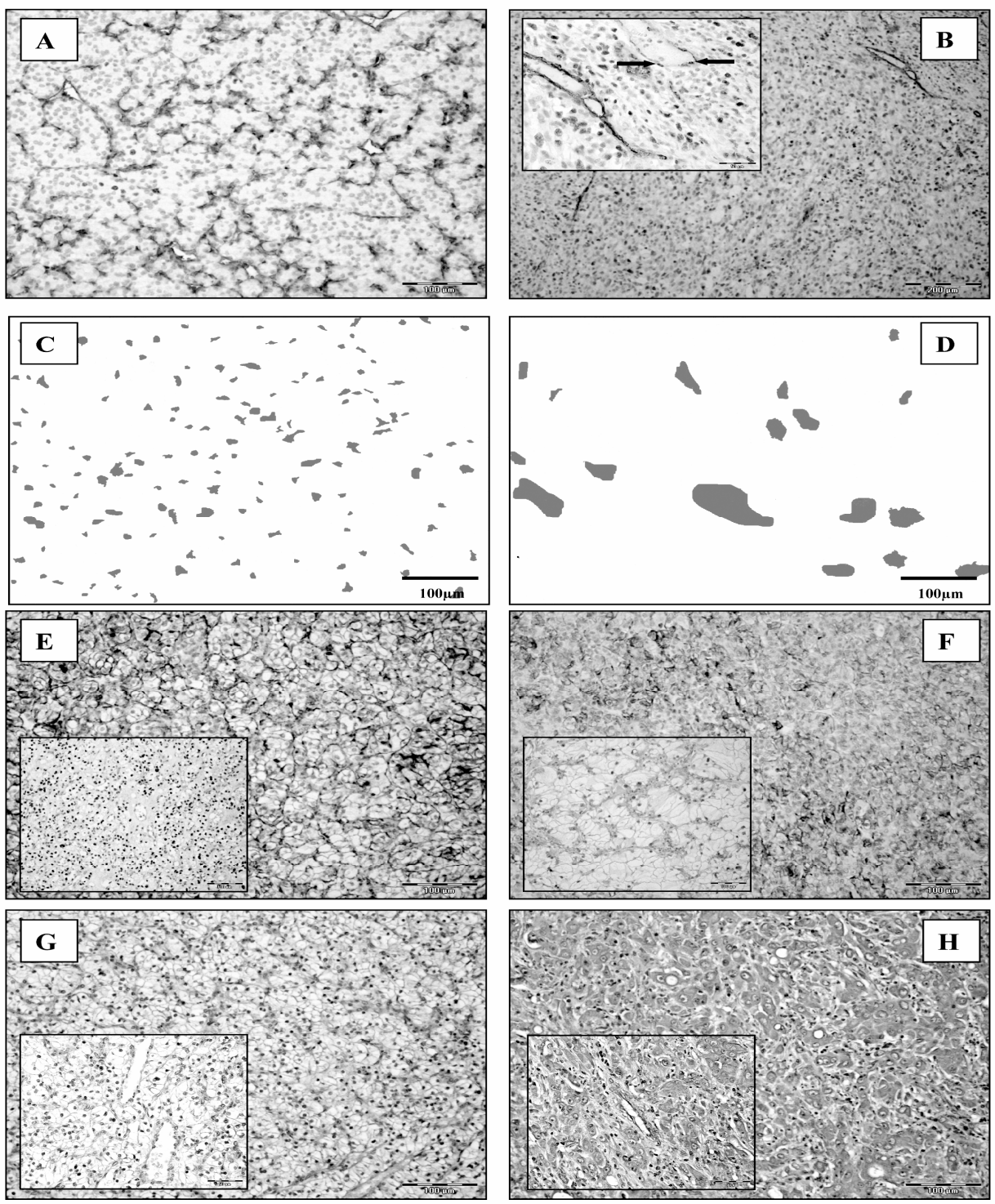

Figure 1: Overview of the immunohistochemical stainings used, in low grade (left: $A, C, E, G$ ) and high grade (right: B,D,F,H) ccRCC. A and B: CD34/Ki-67 IHC double-staining. Black arrows in inset show proliferating Ki67 positive (brown nucleus) endothelial cells (red cytoplasm). C and D: Morphometrical analyses showing an increased vessel area (grey) in high grade ccRCC. E and F : Membranous CA9 staining and nuclear HIF-1 $\alpha$ staining (inset) of tumour cells. Although no significant difference was found between low and high grade tumours for CA9 expression, there was more HIF-1 $\alpha$ expression in low grade ccRCC. $\mathrm{G}$ and $\mathrm{H}$ : predominantly membranous VEGF staining in tumour cells of low grade RCC (G), in contrast with dens cytoplasmatic intratumoural VEGF staining in high grade RCC $(\mathrm{H})$. Insets illustrate also VEGF immunoreactivity of the intratumoural vessels. For full colour picture see page 165. 
Angiogenic activity in clear cell renal cell carcinoma

Table 2: Immunohistochemical and morphometric results of angiogenesis parameters in low grade versus high grade ccRCC

\begin{tabular}{lllllllll|l}
\hline & & MVD & Hotspot & ECP\% & TCP\% & CA9 & HIF & $\begin{array}{l}\text { Vessel } \\
\text { area } \\
\left(\mu m^{2}\right)\end{array}$ & VEGF-A* \\
\hline Low & Min & 44.220 & 52.030 & $0 \%$ & $0 \%$ & 0 & $0 \%$ & 54.99 & $1: 57.5 \%$ \\
grade & Median & $\mathbf{2 3 3 . 8 7 0}$ & $\mathbf{2 9 7 . 8 6 5}$ & $\mathbf{0 . 6} \%$ & $\mathbf{2 . 5} \%$ & $\mathbf{2 8 5}$ & $\mathbf{4 0} \%$ & $\mathbf{1 2 2 . 8 0}$ & $\mathbf{2 : 3 9 . 1 \%}$ \\
RCC & Max & 481.790 & 741.420 & $5.4 \%$ & $30.9 \%$ & 300 & $100 \%$ & $\mathbf{2 2 9 . 8 3}$ & $3: 3.4 \%$ \\
High & Min & 27.580 & 49.430 & $0 \%$ & $0 \%$ & 0 & $0 \%$ & $\mathbf{7 9 . 6 6}$ & $1: 14.3 \%$ \\
grade & Median & $\mathbf{1 5 0 . 8 8 0}$ & $\mathbf{2 5 0 . 5 2 0}$ & $\mathbf{1} \%$ & $\mathbf{5 \%}$ & $\mathbf{2 4 0}$ & $\mathbf{1 5 \%}$ & $\mathbf{1 4 8 . 4 4}$ & $\mathbf{2 : 5 0 . 8 \%}$ \\
RCC & Max & 429.760 & 590.530 & $13.2 \%$ & $46 \%$ & 300 & $95 \%$ & 588.30 & $3: 34.9 \%$ \\
$P$ value & & $<0.001$ & $<0.001$ & 0.049 & 0.009 & 0.262 & 0.002 & 0.017 & $<0.001$ \\
\hline
\end{tabular}

Abbreviations: $\mathrm{N}$, number

p-value: comparison between low grade and high grade RCC (Mann-Whitney)

$*$ : for VEGF-A the percentage of each of the scores is represented

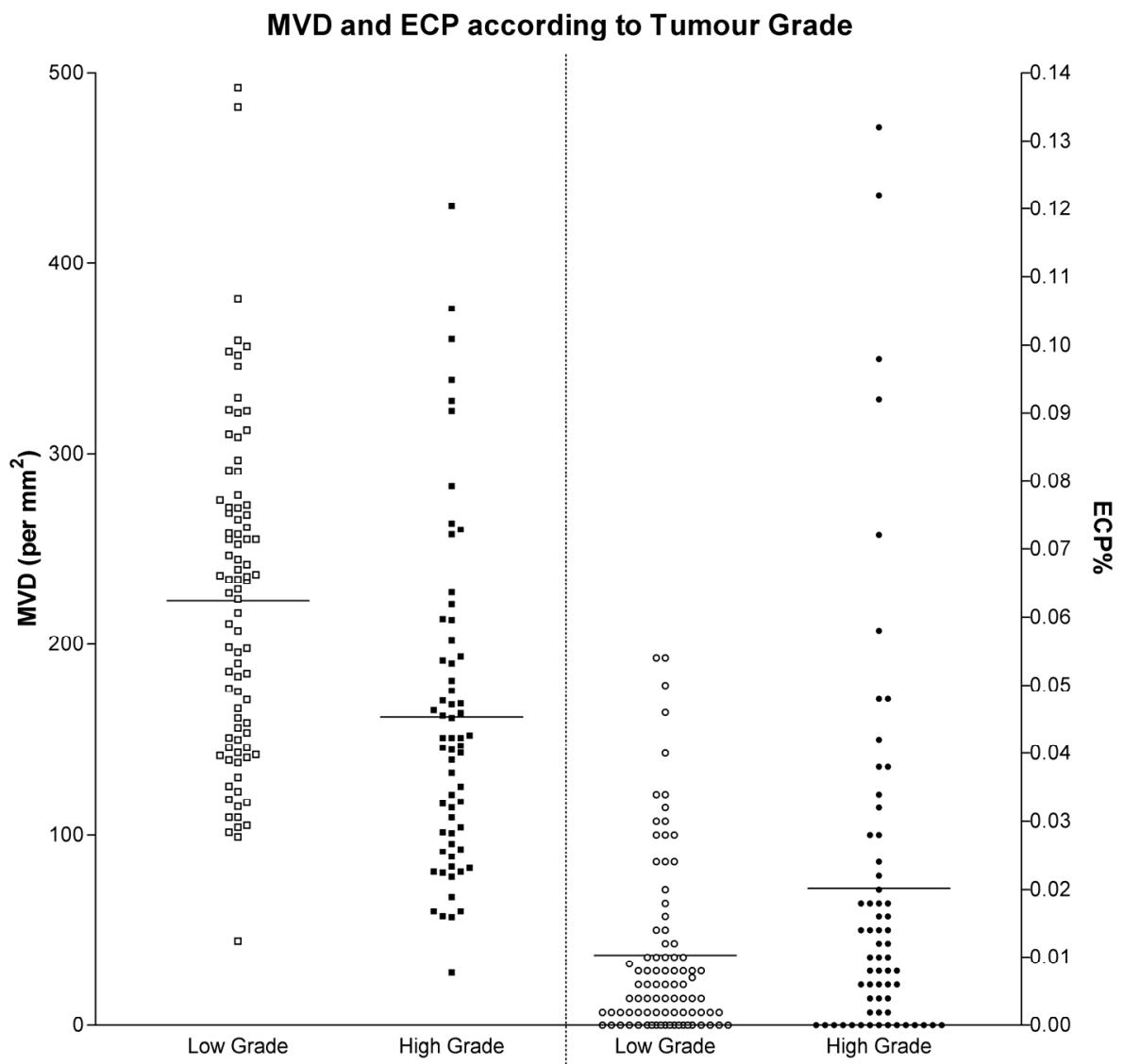

Figure 2: MVD and ECP\% according to tumour grade. ECP\%: endothelial cell proliferation fraction. 
Table 3 and 4 illustrate the expression of angiogenic growth factors/inhibitors in ccRCC and normal renal tissue. In renal tumour tissues there was a significant overexpression of VEGF $(p<0.001)$, VEGF-B $(p=0.004)$, VEGF-C $(p=0.032)$, PLGF $(p<0.001)$, ANG2 $(p<0.001)$, PDGFB1 $(p=0.009)$ and TGF- $\alpha(p<0.001)$ compared to normal renal tissue (Table 3). In contrast, renal cell carcinoma expressed less bFGF $(p<0.001)$ and EGF $(p<0.001)$ than normal renal tissue.

When comparing the PCR data of the angiogenic growth factors in low and high grade tumours (Table 4), only a significant difference could be found in ANG2 expression, which was higher in low grade tumours $(p=0.035)$. Low grade tumours also expressed more VEGFR1 and 2 than high grade $\operatorname{ccRCC}(\mathrm{p}=0.023$ and $\mathrm{p}=0.004$ respectively).

Table 3: Results of PCR quantification of angiogenesis-related genes in normal renal tissue versus ccRCC.

\begin{tabular}{llllllllllll}
\hline & & VEGFA & VEGFB & VEGFC & BFGF & PLGF & ANG1 & ANG2 & EGF & PDGFB1 & TNF $\alpha$ \\
\hline Normal & $\mathrm{N}$ & 16 & 16 & 16 & 16 & 16 & 16 & 16 & 16 & 16 & 16 \\
& Min & 0.055 & 0.012 & 0.003 & 0.092 & 0.002 & 0.007 & 0.001 & 0.004 & 0.009 & 0.000 \\
& Median & $\mathbf{0 . 1 2 4}$ & $\mathbf{0 . 0 2 0}$ & $\mathbf{0 . 0 0 4}$ & $\mathbf{0 . 8 8 2}$ & $\mathbf{0 . 0 0 6}$ & $\mathbf{0 . 0 1 0}$ & $\mathbf{0 . 0 0 3}$ & $\mathbf{0 . 0 4 8}$ & $\mathbf{0 . 0 1 8}$ & $\mathbf{0 . 0 0 1}$ \\
& Max & 0.334 & 0.026 & 0.014 & 1.717 & 0.011 & 0.053 & 0.0138 & 0.268 & 0.057 & 0.011 \\
& & & & & & & & & & & \\
RCC & $\mathrm{N}$ & 33 & 33 & 33 & 33 & 33 & 33 & 33 & 33 & 33 & 33 \\
& Min & 0.091 & 0.008 & 0.001 & 0.002 & 0.007 & 0.000 & 0.006 & 0.000 & 0.008 & 0.000 \\
& Median & $\mathbf{1 . 1 8 7}$ & $\mathbf{0 . 0 3 5}$ & $\mathbf{0 . 0 0 8}$ & $\mathbf{0 . 0 4 5}$ & $\mathbf{0 . 1 1 4}$ & $\mathbf{0 . 0 0 7}$ & $\mathbf{0 . 0 4 5}$ & $\mathbf{0 . 0 0 1}$ & $\mathbf{0 . 0 3 7}$ & $\mathbf{0 . 0 0 2}$ \\
& Max & 4.326 & 0.209 & 0.073 & 0.251 & 1.945 & 0.098 & 0.123 & 0.042 & 0.220 & 0.006 \\
$P$ value & & $<0.001$ & 0.004 & 0.032 & $<0.001$ & $<0.001$ & 0.486 & $<0.001$ & $<0.001$ & 0.009 & 0.703 \\
\hline
\end{tabular}

\begin{tabular}{lllllllll}
\hline & & IL8 & TGF $\boldsymbol{\alpha}$ & TGF $\beta$ & TSP1 & PF4 & VEGFR1 & VEGFR2 \\
\hline Normal & $\mathrm{N}$ & 16 & 16 & 16 & 16 & 16 & 16 & 16 \\
& Min & 0.001 & 0.004 & 0.023 & 0.003 & 0.000 & 0.001 & 0.007 \\
& Median & $\mathbf{0 . 0 0 3}$ & $\mathbf{0 . 0 0 9}$ & $\mathbf{0 . 2 4 1}$ & $\mathbf{0 . 1 5 8}$ & $\mathbf{0 . 0 0 0}$ & $\mathbf{0 . 0 0 8}$ & $\mathbf{0 . 0 1 6}$ \\
& Max & 0.037 & 0.015 & 1.515 & 0.489 & 0.000 & 0.018 & 0.035 \\
RCC & & & & & & & & \\
& N & 33 & 33 & 33 & 33 & 33 & 33 & 33 \\
& Min & 0.000 & 0.000 & 0.041 & 0.007 & 0.000 & 0.000 & 0.002 \\
& Median & $\mathbf{0 . 0 0 7}$ & $\mathbf{0 . 0 3 8}$ & $\mathbf{0 . 2 6 0}$ & $\mathbf{0 . 2 2 7}$ & $\mathbf{0 . 0 0 0}$ & $\mathbf{0 . 0 1 2}$ & $\mathbf{0 . 0 3 0}$ \\
& Max & 0.110 & 0.169 & 1.148 & 2.158 & 0.000 & 0.052 & 0.205 \\
P value & & 0.875 & $<0.001$ & 0.745 & 0.686 & 0.884 & 0.035 & 0.121 \\
\hline
\end{tabular}

Expression values are shown as $2^{-\Delta \mathrm{C}}$

p-value : comparison between normal and RCC (Mann-Whitney) 
Angiogenic activity in clear cell renal cell carcinoma

Table 4: Results of PCR quantification of angiogenesis-related genes in low grade versus high grade ccRCC.

\begin{tabular}{llllllllllll}
\hline & & VEGFA & VEGFB & VEGFC & bFGF & PLGF & ANG1 & ANG2 & EGF & PDGFB1 & TNF $\alpha$ \\
\hline Low & $\mathrm{N}$ & 23 & 23 & 23 & 23 & 23 & 23 & 23 & 23 & 23 & 23 \\
grade & Min & 0.094 & 0.008 & 0.001 & 0.002 & 0.009 & 0.001 & 0.012 & 0.000 & 0.009 & 0.000 \\
RCC & Median & $\mathbf{1 . 1 8 7}$ & $\mathbf{0 . 0 4 4}$ & $\mathbf{0 . 0 0 8}$ & $\mathbf{0 . 0 4 5}$ & $\mathbf{0 . 1 3 0}$ & $\mathbf{0 . 0 0 9}$ & $\mathbf{0 . 0 5 3}$ & $\mathbf{0 . 0 0 1}$ & $\mathbf{0 . 0 4 8}$ & $\mathbf{0 . 0 0 2}$ \\
& Max & 4.326 & 0.209 & 0.073 & 0.250 & 1.945 & 0.098 & 0.123 & 0.034 & 0.220 & 0.006 \\
& & & & & & & & & & & \\
High & $\mathrm{N}$ & 10 & 10 & 10 & 10 & 10 & 10 & 10 & 10 & 10 & 10 \\
grade & Min & 0.091 & 0.008 & 0.003 & 0.009 & 0.007 & 0.000 & 0.006 & 0.000 & 0.008 & 0.000 \\
RCC & Median & $\mathbf{0 . 9 5 3}$ & $\mathbf{0 . 0 2 8}$ & $\mathbf{0 . 0 0 8}$ & $\mathbf{0 . 0 3 4}$ & $\mathbf{0 . 0 3 0}$ & $\mathbf{0 . 0 0 4}$ & $\mathbf{0 . 0 2 7}$ & $\mathbf{0 . 0 0 0}$ & $\mathbf{0 . 0 1 7}$ & $\mathbf{0 . 0 0 2}$ \\
& Max & 4.060 & 0.119 & 0.049 & 0.251 & 0.360 & 0.028 & 0.063 & 0.042 & 0.167 & 0.005 \\
$P$ value & & 0.597 & 0.208 & 0.968 & 0.393 & 0.113 & 0.464 & 0.035 & 0.122 & 0.167 & 0.903 \\
\hline
\end{tabular}

\begin{tabular}{lllllllll}
\hline & & IL8 & TGF $\boldsymbol{\alpha}$ & TGF $\boldsymbol{\beta}$ & TSP1 & PF4 & VEGFR1 & VEGFR2 \\
\hline Normal & $\mathrm{N}$ & 23 & 23 & 23 & 23 & 23 & 23 & 23 \\
& Min & 0.000 & 0.000 & 0.041 & 0.007 & 0.000 & 0.001 & 0.006 \\
& Median & $\mathbf{0 . 0 0 7}$ & $\mathbf{0 . 0 3 8}$ & $\mathbf{0 . 2 3 9}$ & $\mathbf{0 . 1 8 1}$ & $\mathbf{0 . 0 0 0}$ & $\mathbf{0 . 0 1 9}$ & $\mathbf{0 . 0 4 1}$ \\
& Max & 0.045 & 0.169 & 1.148 & 0.757 & 0.000 & 0.052 & 0.205 \\
CC RCC & $\mathrm{N}$ & & & & & & & \\
& Min & 10 & 10 & 10 & 10 & 10 & 10 & 10 \\
& Median & 0.000 & 0.000 & 0.043 & 0.030 & 0.000 & 0.000 & 0.002 \\
& Max & $\mathbf{0 . 0 0 6}$ & $\mathbf{0 . 0 3 8}$ & $\mathbf{0 . 3 9 1}$ & $\mathbf{0 . 2 5 8}$ & $\mathbf{0 . 0 0 0}$ & $\mathbf{0 . 0 0 7}$ & $\mathbf{0 . 0 1 0}$ \\
Pvalue & 0.110 & 0.113 & 0.972 & 2.158 & 0.000 & 0.034 & 0.042 \\
\hline
\end{tabular}

Expression values are shown as $2^{-\Delta \mathrm{Ct}}$

p-value: comparison between low grade and high grade RCC (Mann-Whitney)

\section{2 mRNA expression of angiogenesis-related factors in cCRCC grouped according to median MVD and median ECP\%}

Because of the imperfect association of nuclear grade and ECP\% or MVD (figure 3) and to get more insight in the biology of both vascular patterns related to the inverse association of MVD and ECP\%, ccRCC were also grouped based on low/high MVD (cut off median MVD of 181) and high/low ECP\% (cut off median ECP\% of $0.7 \%$ ). In the cCRCC with high MVD, there was a higher mRNA expresion of VEGF $(p=0.007)$, ANG2 $(p<0.001)$, PLGF $(p=0.007)$ and PDGFB1 $(p=0.001)$. There was also more mRNA expression of VEGFR1 and VEGFR2 in the high MVD group ( $p=0.001$ and $p<0.001)$. A higher expression of ANG1 was found in tumours with low ECP\% $(p=0.008)$. 


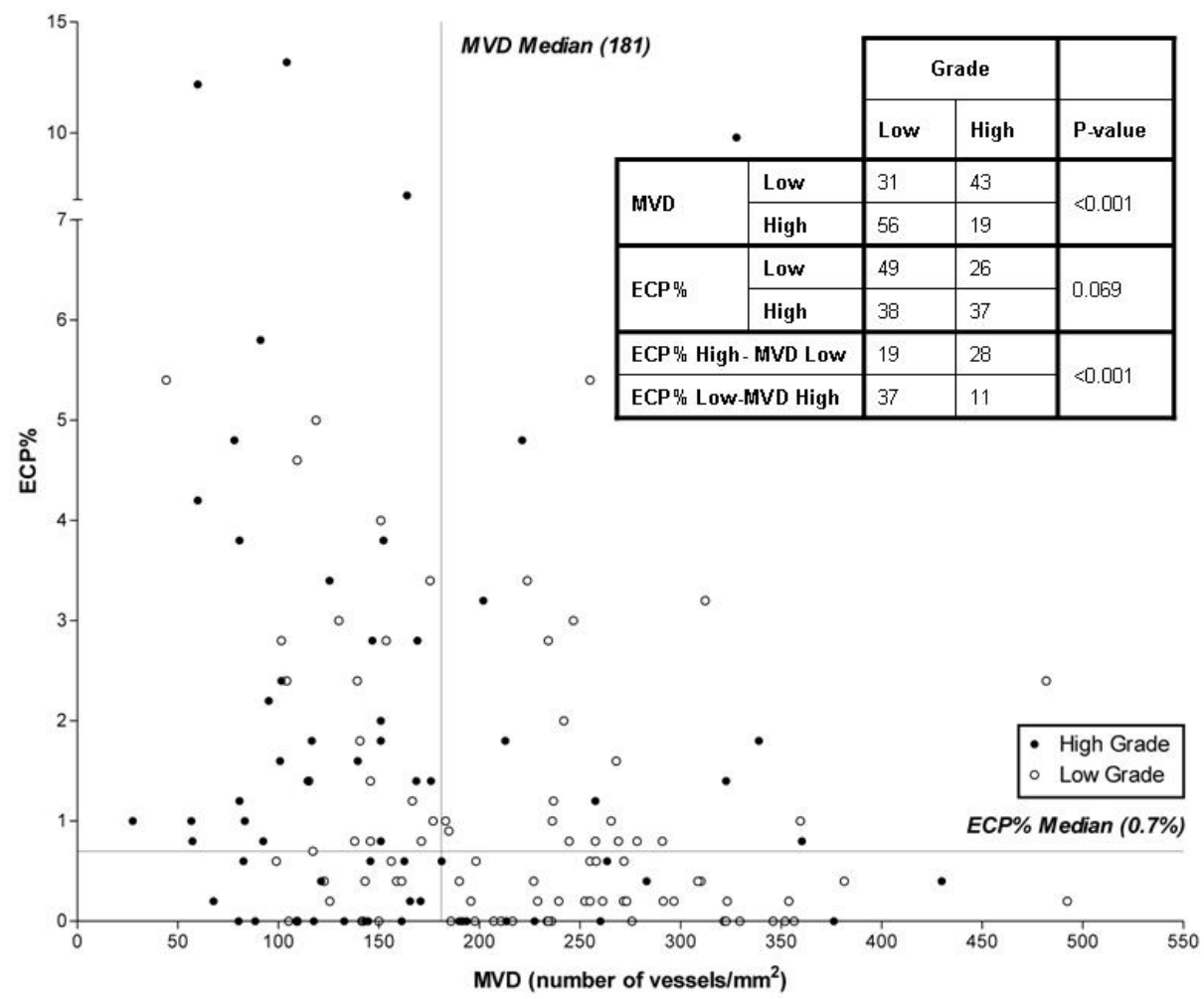

Figure 3: Scatter plot of low grade (open dots) and high grade (filled dots) CcRCC according to median MVD and median ECP\%. Tumours with low ECP\% and high MVD are mainly low grade cCRCC. Tumours with high ECP\% and low MVD are mainly high grade ccRCC.

\section{Discussion}

The interest of our study was to compare angiogenesis parameters in low grade versus high grade ccRCC. In earlier studies MVD was used as a parameter for angiogenesis in RCC and evaluated as a possible prognostic marker, however leading to conflicting results [16-19]. To our knowledge, this is the first study in which the fraction of proliferating endothelial cells (ECP\%) is investigated within RCC.

High grade ccRCCs were characterised by a high ECP\%, which correlated strongly with TCP\%. In spite of the higher ECP\% in high grade cCRCC than the low grade subgroup, MVD was lower in high grade cCRCC. These results suggest that ECP\% is a better indicator for ongoing angiogenesis in ccRCC than MVD. Vessel density reflects intercapillary distance, which is determined by angiogenic factors (stimulators and inhibitors), as well as nonangiogenic factors, such as oxygen and nutrient consumption rates of tumour cells [20]. Tumour progression is associated with a gradual reduction in the in- 
trinsic propensity of cancer cells to undergo apoptosis under a variety of noxious conditions [21]. Cumulative effects of genetic alterations, by means of altered expression of tumour suppressor genes (PTEN, p53 and VHL) and oncogenes (ras,src,..) could affect HIF-1 dependent and independent pathways of cellular respons to hypoxia [22]. In patients with localized RCC p53 appeared to be an independent predictor of tumour progression. [23]. Since a relative decrease of vascular dependence of cancer cells is associated with features of increased malignancy $[24,25]$, tolerance of hypoxic conditions can explain why high grade renal tumours can afford an increased intercapillary distance in comparison with low grade RCC.

In our study, $88 \%$ of all ccRCC showed activation of HIF1- $\alpha$ throughout the whole tumour section, independently of the presence of necrosis. This is consistent with the fact that in $70 \%$ of ccRCC there is a constitutive activation of HIF, through VHL inactivation $^{3}$. Nevertheless differences in HIF-1 $\alpha$ expression were seen between low grade and high grade ccRCC. The lower HIF-1 $\alpha$ expression in high grade $\mathrm{ccRCC}$ could be possibly explained by a progressive switch to HIF-2 $\alpha$ response during tumour progression. An earlier report stated that normal renal epithelium in vivo activates HIF-1 $\alpha$ in response to hypoxia and VHL loss with little or no HIF-2 $\alpha$ [26]. HIF-2 $\alpha$ appears to become progressively more evident than HIF-1 $\alpha$ in foci of renal dysplasia, cyst formation and frank tumours [27]. Since HIF-1 $\alpha$ and HIF-2 $\alpha$ have contrasting properties [28], a possible switch to HIF- $2 \alpha$ could increase the likelihood to proliferation and survival, during tumour evolution.

Evaluation of angiogenesis growth factor/inhibitor expression levels between low and high grade ccRCCs, revealed only a significant difference in ANG2 expression, which was significantly higher in the low grade ccRCC group. ANG2 expression is often induced in endothelia undergoing active remodelling or regression and is up-regulated by hypoxia and several growth factors, including VEGF $[29,30]$. Administration of inhibitors of ANG2 to tumour-bearing mice has been reported to result in delayed tumour growth and reduced endothelial cell proliferation. Therefore, inhibitors of ANG2 may be candidates for clinical development [31].

In our study there is a discrepancy between the immunohistochemical VEGF data and mRNA VEGF expression. Low grade ccRCC with high MVD are characterized by a lower VEGF protein expression and higher mRNA VEGF expression than high grade ccRCC with low MVD. This can be explained by the fact that in the immunohistochemical VEGF staining we only scored cytoplasmatic VEGF positivity in tumour cells, while mRNA VEGF expression also includes VEGF in stroma and at the surface of endothelial cells. In our case, ccRCC with high MVD showed VEGF positive staining of the numerous tumoural vessels (Figure 1).

Within our group of 150 ccRCCs the association of MVD and ECP\% with nuclear grade was however imperfect. Therefore, to get more insight in angiogenesis biology, we compared the mRNA expression levels of angiogenesis-related factors between two subgroups, based on MVD and ECP\% respectively, and leaving Fuhrman grade out of 
account. cCRCC with high MVD revealed a significant higher PLGF and PDGFB1 expression. PLGF, a VEGF-related factor, contributes to tumour angiogenesis by providing increased survival function to endothelial cells [32]. PDGFB is required for recruitment of pericytes and maturation of microvasculature [33]. Furthermore, tumours with low ECP\%, revealed a higher ANG1 expression, which competes with ANG2 for binding of Tie-2 receptor. ANG1 promotes EC sprouting and is essential for maturation and stabilization of the developing vascularization $[34,35]$. These findings suggest that cCRCC with high MVD and low ECP\% may have a more stabilized and mature vasculature than their counterpart. Our finding is concordant with a recent study, which described differentiated and undifferentiated types of blood vessels in ccRCC [36]. They found that an increased density of undifferentiated vessels significantly correlated with higher pathologic grades and shorter patient survival. Since immature vasculature has been shown to be more vulnerable for therapeutic targeting [37], a better response to antiangiogenic drugs might be expected in the high grade ccRCC. Selection of resistant tumour cells can however still occur [38]. On the other hand, anti-VEGF treatment probably has direct effects on tumour cells of ccRCC because these cells express KDR that is phosphorylated, independent of grade [39].

In summary, the results of this suggest that high grade ccRCC have a different angiogenic pattern than low grade $\mathrm{ccRCC}$, with more endothelial cell proliferation in the former tumours, but lower microvessel density, with larger and more immature vessels. These differences in angiogenesis biology might have impact on the effects of anti-angiogenic or anti-VEGF treatment of ccRCC. 


\section{References}

1 Kovacs, G. et al. (1997) The Heidelberg classification of renal cell tumours. J Pathol 183, 131-133

2 Kaelin, W.G., Jr. (2004) The von Hippel-Lindau tumor suppressor gene and kidney cancer. Clin Cancer Res 10, 6290S-6295S

3 Clifford, S.C. et al. (1998) Inactivation of the von Hippel-Lindau (VHL) tumour suppressor gene and allelic losses at chromosome arm $3 p$ in primary renal cell carcinoma: evidence for a VHL-independent pathway in clear cell renal tumourigenesis. Genes Chromosomes Cancer 22, 200-209

4 Wiesener, M.S. et al. (2001) Constitutive activation of hypoxia-inducible genes related to overexpression of hypoxia-inducible factor-1alpha in clear cell renal carcinomas. Cancer Res 61, 5215-5222

5 Semenza, G.L. (1999) Regulation of mammalian O2 homeostasis by hypoxia-inducible factor 1. Annu Rev Cell Dev Biol 15, 551-578

6 Carmeliet, P. and Jain, R.K. (2000) Angiogenesis in cancer and other diseases. Nature 407, 249-257

7 Griffioen, A.W. and Molema, G. (2000) Angiogenesis: potentials for pharmacologic intervention in the treatment of cancer, cardiovascular diseases, and chronic inflammation. Pharmacol Rev 52, 237-268

8 Ruoslahti, E. (2002) Specialization of tumour vasculature. Nat Rev Cancer 2, 83-90

9 Bergers, G. and Benjamin, L.E. (2003) Tumorigenesis and the angiogenic switch. Nat Rev Cancer 3, 401410

10 Konig, B. et al. (1999) The differential expression of proinflammatory cytokines IL-6, IL-8 and TNF-alpha in renal cell carcinoma. Anticancer Res 19, 1519-1524

11 Gelb, A.B. (1997) Renal cell carcinoma: current prognostic factors. Union Internationale Contre le Cancer (UICC) and the American Joint Committee on Cancer (AJCC). Cancer 80, 981-986

12 Van den Eynden, G.G. et al. (2005) Angiogenesis and hypoxia in lymph node metastases is predicted by the angiogenesis and hypoxia in the primary tumour in patients with breast cancer. $\mathrm{Br} \mathrm{J}$ Cancer 93 , 1128-1136

13 Chia, S.K. et al. (2001) Prognostic significance of a novel hypoxia-regulated marker, carbonic anhydrase IX, in invasive breast carcinoma. J Clin Oncol 19, 3660-3668

14 Chakrabarti, J. et al. (2004) The transcription factor DEC1 (stra13, SHARP2) is associated with the hypoxic response and high tumour grade in human breast cancers. Br J Cancer 91, 954-958

15 Thijssen, V.L. et al. (2004) Angiogenesis gene expression profiling in xenograft models to study cellular interactions. Exp Cell Res 299, 286-293

16 Gelb, A.B. et al. (1997) Appraisal of intratumoral microvessel density, MIB-1 score, DNA content, and p53 protein expression as prognostic indicators in patients with locally confined renal cell carcinoma. Cancer 80, 1768-1775

17 Nativ, O. et al. (1998) Clinical significance of tumor angiogenesis in patients with localized renal cell carcinoma. Urology 51, 693-696

18 Sabo, E. et al. (2001) Microscopic analysis and significance of vascular architectural complexity in renal cell carcinoma. Clin Cancer Res 7, 533-537

19 Rioux-Leclercq, N. et al. (2001) Clinical significance of cell proliferation, microvessel density, and CD44 adhesion molecule expression in renal cell carcinoma. Hum Pathol 32, 1209-1215

20 Hlatky, L. et al. (2002) Clinical application of antiangiogenic therapy: microvessel density, what it does and doesn't tell us. J Natl Cancer Inst 94, 883-893

21 Bedi, A. et al. (1995) Inhibition of apoptosis during development of colorectal cancer. Cancer Res 55 1811-1816

22 Semenza, G.L. (2000) Hypoxia, clonal selection, and the role of HIF-1 in tumor progression. Crit Rev Biochem Mol Biol 35, 71-103 
23 Shvarts, O. et al. (2005) p53 is an independent predictor of tumor recurrence and progression after nephrectomy in patients with localized renal cell carcinoma. J Urol 173, 725-728

24 Yu, J.L. et al. (2001) Heterogeneous vascular dependence of tumor cell populations. Am J Pathol 158, 1325-1334

25 Carmeliet, P. et al. (1998) Role of HIF-1alpha in hypoxia-mediated apoptosis, cell proliferation and tumour angiogenesis. Nature 394, 485-490

26 Rosenberger, C. et al. (2002) Expression of hypoxia-inducible factor-1alpha and -2alpha in hypoxic and ischemic rat kidneys. J Am Soc Nephrol 13, 1721-1732

27 Mandriota, S.J. et al. (2002) HIF activation identifies early lesions in VHL kidneys: evidence for sitespecific tumor suppressor function in the nephron. Cancer Cell 1, 459-468

28 Raval, R.R. et al. (2005) Contrasting properties of hypoxia-inducible factor 1 (HIF-1) and HIF-2 in von Hippel-Lindau-associated renal cell carcinoma. Mol Cell Biol 25, 5675-5686

29 Oh, H. et al. (1999) Hypoxia and vascular endothelial growth factor selectively up-regulate angiopoietin2 in bovine microvascular endothelial cells. J Biol Chem 274, 15732-15739

30 Mandriota, S.J. et al. (2000) Hypoxia-inducible angiopoietin-2 expression is mimicked by iodonium compounds and occurs in the rat brain and skin in response to systemic hypoxia and tissue ischemia. Am J Pathol 156, 2077-2089

31 Oliner, J. et al. (2004) Suppression of angiogenesis and tumor growth by selective inhibition of angiopoietin-2. Cancer Cell 6, 507-516

32 Adini, A. et al. (2002) Placental growth factor is a survival factor for tumor endothelial cells and macrophages. Cancer Res 62, 2749-2752

33 Lindahl, P. et al. (1997) Pericyte loss and microaneurysm formation in PDGF-B-deficient mice. Science $277,242-245$

34 Distler, J.H. et al. (2003) Angiogenic and angiostatic factors in the molecular control of angiogenesis. $Q \mathrm{~J}$ Nucl Med 47, 149-161

35 Papapetropoulos, A. et al. (1999) Direct actions of angiopoietin-1 on human endothelium: evidence for network stabilization, cell survival, and interaction with other angiogenic growth factors. Lab Invest 79 , 213-223

36 Yao, X. et al. (2007) Two distinct types of blood vessels in clear cell renal cell carcinoma have contrasting prognostic implications. Clin Cancer Res 13, 161-169

37 Feron, O. et al.(2004) Targeting the tumor vascular compartment to improve conventional cancer therapy. Trends Pharmacol Sci, 25, 536-42.

$38 \mathrm{Yu}$, J.L. et al. (2002). Effect of p53 status on tumor response to antiangiogenic therapy. Science, 295, $1526-8$

39 Fox, S.B.et al (2004) Phosphorylated KDR is expressed in the neoplastic and stromal elements of human renal tumours and shuttles from cell membrane to nucleus. J Pathol, 202, 313-20. 


\section{Chapter 5}

\section{Different angiogenic potential in low and high grade sporadic clear cell renal cell carcinoma is not related to alterations in the von Hippel-Lindau gene}

Marcella M Baldewijns, Iris JH van Vlodrop, Kim M Smits, Peter B Vermeulen, Gert G Van den Eynden, Fiona Schot, Tania Roskams, Hein van Poppel, Manon van Engeland and Adriaan P de Bruïne

Cellular Oncology 2009, in press 


\begin{abstract}
von Hippel-Lindau (VHL) inactivation is common in sporadic clear cell renal cell carcinomas (ccRCC). pVHL is part of the ubiquitin ligase complex that targets the alpha subunits of hypoxia-inducible transcription factor (HIF) for degradation under welloxygenated conditions. In the absence of wild-type pVHL, as observed in VHL patients and most sporadic ccRCCs, constitutive upregulation of HIF results in transcriptional activation of angiogenesis-related genes, such as VEGF. Differences in angiogenic activity within the group of ccRCCs were reported and strong genotype-phenotype correlations were found in patients with VHL disease, raising a question about the importance of $\mathrm{VHL}$ inactivation status in angiogenic behaviour and tumour progression.

To address this question, we investigated the influence of VHL mutation (direct sequencing)/hypermethylation (methylation-specific PCR) on angiogenesis/tumour parameters (immunohistochemistry) in 150 patients with sporadic ccRCC.

We found no significant association between VHL mutation or methylation and angiogenesis/tumour parameters.

These data indicate that tumour progression and angiogenesis are not directly influenced by VHL alterations and that additional genetic/epigenetic events should be considered to explain the diverse angiogenic and proliferative behaviour during tumour progression.
\end{abstract}




\section{Introduction}

The most widely observed genetic aberration in clear cell RCC (ccRCC) is loss of chromosome 3p, harbouring the von Hippel-Lindau (VHL) tumour suppressor gene, which is located on chromosome 3 p25 and plays an important role in hereditary and sporadic ccRCC [1]. 50\%-75\% of sporadic ccRCCs show biallelic VHL inactivation [1-3]. The VHL gene exists of three exons and VHL mutations are heterogeneous distributed throughout the coding sequence, except that intragenic missense mutations are rarely seen within the first 50 codons [4]. In 5-20\% of reported cases the VHL gene is silenced by promoter hypermethylation [5-8]. The best characterized function of $\mathrm{pVHL}$ is its role as a component of the ubiquitin E3 ligase complex that targets hypoxia inducible factor HIF- $\alpha$ for ubiquitin-dependent proteasome degradation $[9,10]$. The $\alpha$-subunits of HIF are rapidly degraded by the proteasome under normal conditions, but are stabilized by hypoxia [11]. In hypoxic cells and in RCC, lacking functional pVHL, HIF- $\alpha$ degradation is suppressed, leading to enhanced transcription of target genes, including genes involved in cell proliferation, apoptosis, extra cellular matrix formation, energy metabolism and pro-angiogenic genes such as vascular endothelial growth factor (VEGF) contributing to the activation of the angiogenic switch [1,12-15]. Therefore it can be hypothesized that a difference in angiogenesis/hypoxia can be expected in ccRCC patients with versus without functional VHL.

Previously, we observed significant differences in angiogenesis and hypoxia parameters between low grade (Fuhrman grade 1-2) and high-grade (Fuhrman grade 3-4) cCRCC [16].

Since strong genotype-phenotype correlations were found in patients with VHL disease, whereby the tumour phenotype was influenced by the nature of VHL mutations [17], the question emerges whether VHL alterations can explain the different angiogenic phenotypes and different biological behaviour in sporadic ccRCC.

Here, we examine the influence of VHL alterations (mutation/promoter hypermethylation) on angiogenesis and hypoxia parameters in cCRCC and correlate these results with clinical outcome. To the best of our knowledge, this is the first study investigating the association between VHL alterations and active angiogenesis, reflected by endothelial cell proliferation measurement.

\section{Material and methods}

\subsection{Patients and tissue samples}

Tumour samples of 150 patients with sporadic $\mathrm{CCRCC}$, treated with radical or partial nephrectomy without adjuvant treatment, have been collected retrospectively. The samples were derived from the archives of the Departments of Histopathology of the University Hospitals of Leuven and Maastricht. The original histological slides, stained 
with hematoxylin and eosin, were reviewed to confirm nuclear Fuhrman grading. All patients were evaluated post-operatively at regular intervals by means of physical examination, chest ray, abdominal computed tomography or ultrasound. When indicated, a bone scan was performed. The median follow-up time was 64 months (range 1-153 months) and mean follow-up of 9 years. The clinico-pathological data of the study population are summarized in Table 1.

Table 1: Clinical and histopathological characteristics

\begin{tabular}{l|l}
\hline Gender & Clinicopathological data (N = 150) \\
male/female & $99 / 51(66 / 34 \%)$ \\
Age $^{a}$ (years) & $66.6( \pm 12.1$ s.d.) \\
Mean tumour diameter (cm) & $6.36( \pm 3.45$ s.d.) \\
Fuhrman grade & $15(10 \%)$ \\
1 & $72(48 \%)$ \\
2 & $46(30.7 \%)$ \\
3 & $17(11.3 \%)$ \\
4 & \\
Tumour stage ${ }^{a}$ & $82(54.7 \%)$ \\
I & $24(16 \%)$ \\
II & $42(28 \%)$ \\
III & $2(1.3 \%)$ \\
IV & \\
Lymph node status & \\
1 & 4 \\
2 & 11 \\
\hline a T and N status were assigned according to the TNM classification of the International Union Against cancer \\
2002
\end{tabular}

\subsection{VHL mutation analysis}

Tumour tissue was selected on the basis of haematoxylin and eosin stained sections to ensure a minimum of $80 \%$ tumour cells in the samples. Genomic DNA was extracted from $20 \mu \mathrm{m}$ slides from each case. After dewaxing of the tumour slides in xylene and rehydration through sequential changes of alcohol, DNA was isolated and purified with Puregene $^{\circledast}$ DNA purification kit (BIOzym, Landgraaf, The Netherlands). PCR primers used for amplification are available upon request. DNA was amplified using a (multiplex) semi-nested PCR approach. The first (multiplex) PCR was performed using $200 \mathrm{ng}$ of the extracted DNA and subjected to 35 cycles of PCR: $30 \mathrm{sec}$ at $94^{\circ} \mathrm{C}, 30 \mathrm{sec}$ at $66^{\circ} \mathrm{C}$ and $30 \mathrm{sec}$ at $72^{\circ} \mathrm{C}$. The second PCR with $4 \mu$ diluted (1:100) PCR product was subjected to 35 cycles of PCR: $30 \mathrm{sec}$ at $94^{\circ} \mathrm{C}, 30 \mathrm{sec}$ at Tm and $30 \mathrm{sec}$ at $72^{\circ} \mathrm{C}$. Exon amplification was performed in $25 \mu \mathrm{l}$ (1x PCR buffer, $0.2 \mathrm{mM}$ dNTP, $1.5 \mathrm{mM} \mathrm{MgCl}, 0.2 \mu \mathrm{l}$ Primer (each), 1 unit Platinum ${ }^{\circledR}$ Taq DNA polymerase (Invitrogen ${ }^{\text {TM }}$, Breda, The Netherlands) and template DNA). The products were analyzed by agarose gel electrophoresis and visualized by Gelstar ${ }^{\circledast}$ (Cambrex, New Jersey, USA).

For direct sequence analysis, the PCR products were purified using multiscreen ${ }^{{ }_{H}}{ }_{\text {HTs }} 96$ wells plate (Millipore, Billerica, USA) and collected in $50 \mu \mathrm{l}$ sterile water. For the sequence reaction, $2 \mu \mathrm{l}$ purified DNA, BigDye vl.1, sequence buffer and primers (3.2 
pmol) were used. The PCR was subjected to 25 cycles of PCR: $10 \mathrm{sec}$ at $96^{\circ} \mathrm{C}, 5 \mathrm{sec}$ at $50^{\circ} \mathrm{C}$ and $4 \mathrm{~min}$ at $60^{\circ} \mathrm{C}$. The sequence products were precipitated with ethanol in a 96 well plate and dissolved in $20 \mu \mathrm{l}$ sterile water and measured with the ABI 3710 Genetic Analyzer. Mutations were identified by Mutation Surveyor DNA Variant Analysis Software version 3.0 (Soft Genetics LLC, USA) and checked manually. VHL mutation analysis succeeded in 127 of 150 tumour samples.

\subsection{VHL promoter $C p G$ island methylation analysis}

DNA methylation in the CpG island of the VHL gene promoter was determined by chemical modification of genomic DNA with sodium bisulfite and subsequent methylation-specific PCR (MSP) as described in detail elsewhere $[18,19]$. Briefly, $0.5 \mu \mathrm{g}$ of DNA was modified by sodium bisulfite using the EZ DNA methylation kit (Zymo Research) according to the manufacturer's instruction. To facilitate MSP analysis on DNA retrieved from formalin-fixed, paraffin embedded tissue, DNA was first amplified with flanking PCR primers that amplify bisulfite-modified DNA but do not preferentially amplify methylated or unmethylated DNA. The resulting fragment was used as a template for the VHL MSP reaction. Primer sequences and PCR conditions are available upon request.

All PCRs were performed with controls for unmethylated alleles (DNA from normal lymphocytes), methylated alleles (normal lymphocyte DNA treated in vitro with Sssl methyltransferase (New England Biolabs)), and a control without DNA. Ten $\mu$ l of each MSP reaction were directly loaded onto $2 \%$ agarose gels, stained with Gelstar (Cambrex), and visualized under UV illumination. MSP reactions succeeded in 134 of 150 tumour samples. To assess reproducibility, MSP reactions have been performed in duplicate starting from DNA amplification with flanking PCR primers. The reproducibility was $96 \%$.

\subsection{Assessment of angiogenesis and hypoxia parameters}

Serial sections of 4 micron were made from the paraffin embedded kidney tumours. Methods for quantification of microvessel density (MVD), endothelial cell proliferation, tumour cell proliferation and assessment of HIF-1 $\alpha$, carbonic anhydrase IX (CAIX), VEGF expression are described more in detail elsewhere [16].

Briefly, by means of a CD34/Ki67 double-stain procedure[20] MVD, the fraction of proliferating endothelial cells and tumour cells were evaluated. One section was analyzed per tumour. For assessment of the vascular density within a tumour section, 1 hotspot (most vascularised microscopic field) was selected and 4 areas were chosen randomly. Vessel counts were done at 200x magnification using an optical grid. The presence of a vascular lumen was not necessary to identify a microvessel. MVD was expressed as vessels per $\mathrm{mm}^{2}$. Since, the mean vessel density of the tumour gives an estimate of the net result of phases of angiogenesis and angioregression a tumour went through, we also assessed the fraction of proliferating endothelial cells, which is 
a better measure for active angiogenesis. A total number of approximately 500 intratumoural endothelial cells and 500 tumour cells were evaluated on consecutive fields at a 400x magnification and the fractions of proliferating endothelial cells (ECP\%) and tumour cells (TCP\%) were assessed. ECP\% and TCP\% were calculated according to the following formulas: $\mathrm{ECP} \%=$ (the number of endothelial cells with Ki67-stained nuclei/total number of endothelial cells evaluated) $\times 100 ;$ TCP\% = (the number of tumour cells with Ki67-stained nuclei/total number of tumour cells evaluated) $\times 100$.

For CAIX immunohistochemical staining [20], a score of 0-3 for intensity of staining within the tumour was given (0:no staining, 1:weak staining, 2:moderate staining, 3 :strong staining). The percentage of immunostained tumour cells was estimated. The product (intensity score $x$ the percentage of immunoreactive tumour cells) yielded a final score of 0-300.

The HIF-1 $\alpha$ immunohistochemical staining was carried out as described elsewhere [20]. For HIF-1 $\alpha$ expression analysis, only tumour cells with completely and darkly stained nuclei were scored as positive. The fraction of HIF-1 $\alpha$-positive tumour cells was estimated.

Furthermore, a VEGF immunohistochemical staining was performed [16] and VEGF expression was semi-quantitatively assessed according to a four point grading scale: 0 absence of tumour staining, 1+ membrane staining tumour cells, 2+ strong membrane staining and cytoplasmic staining of $<50 \%$ of the tumour cells and $3+$ strong cytoplasmic staining in $>50 \%$ of all tumour cells.

The assessments of all the higher mentioned immunohistochemical stainings were made by two independent observers (inter-observer variability $<5 \%$ ).

\section{Data analysis}

For statistical analysis, ccRCCs were first categorized according to presence or absence of loss of function mutations (defined as truncating mutations, predicted to alter the open reading frame, including nonsense mutations and frameshift mutations) on the one hand and presence or absence of VHL promoter methylation on the other hand. Secondly ccRCCs were subdivided into a group with no mutations or silent mutations, a second group with loss of function mutations and a third group with mutations of unknown biological significance (missense mutations without abrogating the VHL start codon, in-frame mutations). Thirdly, ccRCCs were categorized according to presence or absence of $\mathrm{VHL}$ changes, by which $\mathrm{VHL}$ change was defined as a loss of function mutation and/or VHL promoter methylation.

Since angiogenesis and hypoxia parameters were not normally distributed, nonparametric statistics were used to compare continuous variables (e.g. vessel density, endothelial or tumour cell proliferation, percentage of HIF-1 $\alpha$ immunoreactive tumour cells, CAIX score, VEGF score) between tumours with different VHL mutation or methylation status. To compare two groups Mann-Whitney and more than two groups 
Kruskall-Wallis tests were used, respectively. For the association between noncontinuous variables (e.g. Fuhrman grade, tumour stage) and VHL mutation or methylation status, a Chi-square test was used. The above mentioned statistical analyses were performed using the SPSS 12.0. software package.

The survival analyses were performed by use of the statistical package STATA 10.0.

Cause-specific overall survival (OS) was defined as the time from nephrectomy until renal cancer-related death or until the end of follow-up. Metastatic-free survival (MFS) was defined as the time from nephrectomy until the occurrence of a metastasis or until the end of follow-up. Kaplan-Meier curves and log-rank tests were used to estimate the overall influence of VHL loss of function mutations, VHL promoter hypermethylation and VHL changes on OS and MFS. Hazard ratios (HRs) and corresponding 95\%-confidence intervals $(\mathrm{Cl})$ were assessed by use of Cox proportional hazard models. Factors were considered possible confounders if they were known prognostic factors for renal cancer and influenced the crude HR. Confounders that were eventually in cluded in the model were sex, age at diagnosis, cancer stage, tumour size $(\mathrm{cm})$, Fuhrman grading, endothelial cell proliferation, tumour cell proliferation, HIF-1 $\alpha$, VEGF score and vessel density. CA IX score was evaluated as a possible confounder, however, was observed to have no influence, therefore CAIX score was not included in the model. As the number of stage IV cancers was small $(n=2)$, we decided to exclude these cases from the analyses that were adjusted for cancer stage. The proportional hazard assumption was tested using the Schoenfeld residuals.

In all statistical analyses all quoted $p$-values are two sided, and p-value $\leq 0.05$ was considered statistically significant.

\section{Results}

\subsection{VHL mutation and promoter CpG island methylation is mutual exclusive in CCRCC}

Eighty-seven of 127 ccRCCs (68\%) showed VHL mutations. In the 87 VHL mutant ccRCCs, 108 mutations were observed. Seventeen ccRCC cases had two mutations and two ccRCC cases showed three mutations. Figure 1 shows the type of mutation plotted against the codon number. Except for one single nucleotide polymorphism at codon 25 and silent mutation at codon 40 , no further mutations were seen within the first 57 codons.

Forty-three of 108 (39.8\%) VHL mutations were missense mutations, 45/108 (41.7\%) were truncating mutations (of which 38 frameshift and 7 nonsense mutations), 17/108 (15.7\%) were silent mutations and 2 (1.9\%) were in-frame deletions. Forty-seven of 108 (43.5\%) mutations affected exon 1, 36/108 (33.3\%) mutations were located within exon 2 and 25/108 (23.1\%) mutations were detected within exon 3. Deletions were more common in exon $1(n=47)$ than exon $2(n=36)$ and exon $3(n=25)$. 
In 11 of 134 (8\%) ccRCCs methylation of the VHL promoter CpG island was present. In 38 of 134 ccRCCs (28\%) mutations nor methylation of the VHL promoter CpG island was found. Both the frequencies of VHL mutations and VHL promoter methylation, as well as types of mutations are in accordance with ranges described in literature [1-3,5$7,21]$.
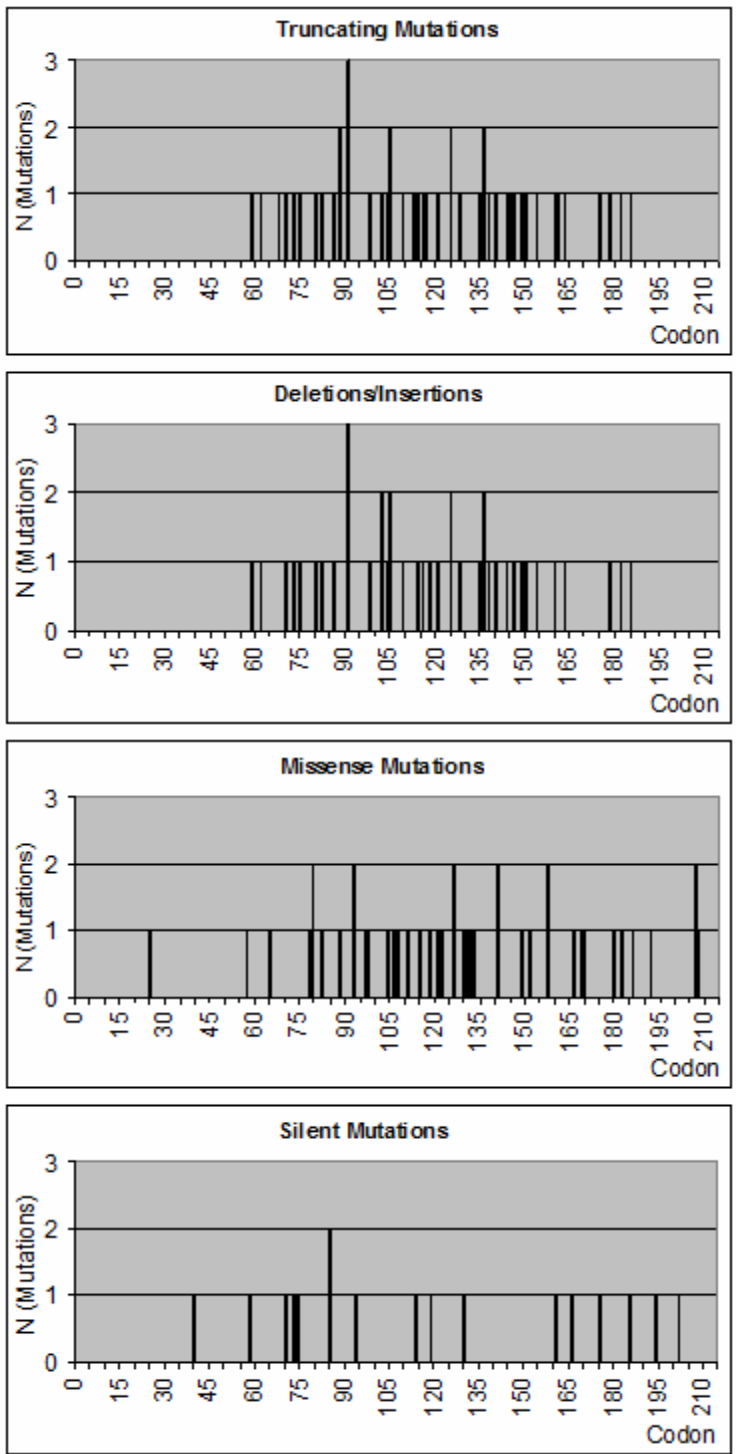

Figure 1: Type of mutation plotted against the codon number. Codon 1-114 encodes exon 1, codon 114-155 encodes exon 2 and codon 155-213 encodes exon 3. 
Different angiogenic potential

Table 2 shows that VHL mutation and VHL promoter methylation are almost mutually exclusive.

Table 2a: $\quad$ The presence of VHL loss of function mutations in relation to VHL promoter methylation

\begin{tabular}{llll}
\hline$V H L$ mutation & \multicolumn{2}{l}{ VHL promoter methylation } & Total \\
\hline 0 & Absent & present & 46 \\
1 & 38 & 8 & 45 \\
Total & 44 & 1 & 91 \\
\hline $0:$ no & 82 & 9 & 9
\end{tabular}

0: no mutation or silent mutation, 1: loss of function mutation (frameshift, nonsense)

Pearson chi2 $=5.8732 \operatorname{Pr}=0.015$

Table $\mathbf{2 b}$ : The presence of $\mathrm{VHL}$ mutations in relation to $\mathrm{VHL}$ promoter methylation

\begin{tabular}{|c|c|c|c|}
\hline \multirow{2}{*}{ VHL mutation } & \multicolumn{3}{|c|}{ VHL promoter methylation } \\
\hline & Absent & present & Total \\
\hline 0 & 38 & & 46 \\
\hline 1 & 44 & & 45 \\
\hline 2 & 34 & & 36 \\
\hline Total & 1161 & & 127 \\
\hline
\end{tabular}

0: no mutation or silent mutation, 1: loss of function mutation, 2: mutation of unknown biological significance (missense mutations without abrogating the VHL start codon, in-frame mutations) Pearson chi2 $=7.2287 \operatorname{Pr}=0.027$

\subsection{Immunohistochemical results}

As reported previously [16], high-grade CCRCC showed a significant higher ECP\% (P = 0.049), higher TCP\% $(P=0.009)$, higher VEGF protein expression $(P<0.001)$, lower MVD $(P<0.001)$ and lower HIF-1 $\alpha$ protein expression $(P=0.002)$ than low grade cCRCC. No significant differences were seen in CA-IX expression between both groups. Furthermore, a strong positive correlation was found between ECP\% and TCP\% (Rho=0.82, $p<0.001$ ).

\subsection{Alterations in the VHL gene do not influence tumour or angiogenesis/hypoxia parameters}

No significant differences were observed between the presence/absence of VHL promoter methylation and/or mutations when examined with regard to Fuhrman grade, tumour stage, TCP\%, angiogenesis and hypoxia related parameters (MVD, ECP\%, VEGF protein expression, HIF-1 $\alpha$ protein expression) (table 3-4). The type of VHL mutation was not significantly associated with angiogenesis/hypoxia and clinical variables (table 4). Only a significant correlation was present between CA-IX expression and VHL change (table 4). 
Table 3: Tumour parameters stratified by VHL mutation/methylation status

\begin{tabular}{|c|c|c|c|c|c|c|c|c|c|}
\hline & \multirow[t]{2}{*}{$\mathrm{N}$} & \multirow[t]{2}{*}{$\begin{array}{l}\text { No } \\
\text { mutation }\end{array}$} & \multirow[t]{2}{*}{$\begin{array}{l}\text { Silent } V H L \\
\text { mutation }\end{array}$} & \multicolumn{2}{|c|}{$\begin{array}{l}\text { Loss of function } \\
V H L \text { mutation }\end{array}$} & \multicolumn{2}{|c|}{$\begin{array}{l}\text { VHL mutation of } \\
\text { unknown biological } \\
\text { significance }\end{array}$} & \multirow[t]{2}{*}{$\begin{array}{l}\text { VHL promoter } \\
\text { methylation } \\
\text { absent }\end{array}$} & \multirow[t]{2}{*}{$\begin{array}{l}\text { VHL promoter } \\
\text { methylation } \\
\text { present }\end{array}$} \\
\hline & & & & & & & & & \\
\hline 1 & 12 & 7 (58\%) & $0(0 \%)$ & 2 & $(16 \%)$ & 3 & $(25 \%)$ & 11 (92\%) & $1(8 \%)$ \\
\hline 2 & 64 & $16(25 \%)$ & $5(8 \%)$ & 23 & (35\%) & 18 & $(28 \%)$ & 58 (91\%) & $6(9 \%)$ \\
\hline 3 & 43 & $15(35 \%)$ & $10(23 \%)$ & 15 & (35\%) & 10 & $(23 \%)$ & 40 (93\%) & $3(7 \%)$ \\
\hline 4 & 15 & $5(33 \%)$ & $2(13 \%)$ & 5 & (33\%) & 5 & (33\%) & 14 (93\%) & $1(7 \%)$ \\
\hline \multicolumn{10}{|c|}{ Stage } \\
\hline 1 & 73 & $26(36 \%)$ & 7 (9\%) & 22 & $(30 \%)$ & 18 & $(25 \%)$ & 64 (88\%) & $9(12 \%)$ \\
\hline 2 & 23 & $8(35 \%)$ & $1(4 \%)$ & 7 & $(30 \%)$ & 7 & $(30 \%)$ & 22 (96\%) & $1(4 \%)$ \\
\hline 3 & 37 & $8(22 \%)$ & 9 (24\%) & 16 & (43\%) & 11 & (30\%) & 36 (97\%) & $1(3 \%)$ \\
\hline 4 & 1 & $1(100 \%)$ & $0(0 \%)$ & 0 & $(0 \%)$ & 0 & $(0 \%)$ & $1(100 \%)$ & $0(0 \%)$ \\
\hline
\end{tabular}

Table 4: Relationship between VHL alterations and angiogenesis/tumour parameters

\begin{tabular}{|c|c|c|c|c|}
\hline & $\begin{array}{l}\text { 1. VHL promoter } \\
\text { methylation } \\
(\mathrm{n}=134) \\
0=123 ; 1=11 \\
p \text {-value }\end{array}$ & $\begin{array}{l}\begin{array}{l}\text { 2. VHL mutation } \\
(\mathrm{n}=91) \\
0=46 ; 1=45 \\
p \text {-value }\end{array}\end{array}$ & $\begin{array}{l}\text { 3. VHL mutation } \\
(\mathrm{n}=127) \\
0=.46 ; 1=45 ; 2=36 \\
p \text {-value }\end{array}$ & $\begin{array}{l}\begin{array}{l}\text { 4. VHL change } \\
(\mathrm{n}=93) \\
0=38 ; 1=55 \\
p \text {-value }\end{array} \\
\end{array}$ \\
\hline \multicolumn{5}{|c|}{ Angiogenesis parameters } \\
\hline MVD & 0.277 & 0.499 & 0.798 & 0.937 \\
\hline ЕСР\% & 0.300 & 0.817 & 0.464 & 0.248 \\
\hline HIF-1 $1 \alpha$ & 0.789 & 0.151 & 0.255 & 0.150 \\
\hline CAIX & 0.909 & 0.087 & 0.150 & 0.047 \\
\hline VEGF & 0.650 & 0.267 & 0.503 & 0.147 \\
\hline \multicolumn{5}{|l|}{ Tumour parameters } \\
\hline ТСР\% & 0.113 & 0.817 & 0.867 & 0.409 \\
\hline Fuhrman grade (1-4) & 0.686 & 0.762 & 0.944 & 0.953 \\
\hline Tumour stage (1-4) & 0.108 & 0.212 & 0.424 & 0.437 \\
\hline
\end{tabular}

1.VHL promoter methylation: $0=$ unmethylated; $1=$ methylated

2.VHL mutation : $0=$ no or silent mutation; 1 = loss of function mutation

3.VHL mutation : 0 = no or silent mutation; 1 = loss of function mutation; $2=$ mutation of unknown significance

4.VHL change: $0=$ no methylation and/or loss of function mutation; $1=$ VHL methylation and/or loss of function mutation

MVD: microvessel density; ECP\%: endothelial cell proliferation fraction; TCP\%: tumour cell proliferation fraction.

\subsection{Alterations in the VHL gene do not influence clinical outcome}

The median OS was 64 months (range 1-153) and the median MFS was 56 months (range 0-153). Median OS in the patients with VHL loss of function mutations was 63 months, median OS in patients with VHL wildtype was 61.4 months. Median MFS in the patients with VHL loss of function mutations was 54 months, median MFS in patients with VHL wildtype was 56 months.

Kaplan-Meier curves showed no statistically significant difference in OS for VHL loss of function mutations (log-rank $p=0.552$ ), VHL mutations (including loss of function mutations and mutations of unknown biological significance, log-rank $p=0.489$ ) or VHL changes (log-rank $p=0.424$ ) (Figure $2 a, c, e)$. For MFS no statistically significant differ- 
ences were observed for VHL loss of function mutations (log-rank $p=0.661)$, VHL mutations (including loss of function mutations and mutations of unknown biological significance, log-rank $p=0.758$ ) or VHL changes (log-rank $p=0.400$ ) either (Figure $2 b, d, f)$.

Results from the age and sex adjusted Cox proportional hazard analyses did not show a statistically significant influence of VHL mutation (HR 1.18, 95\%-Cl 0.57-2.44), VHL promoter methylation (HR $0.89,95 \%-\mathrm{Cl} 0.27-2.95$ ) or VHL changes (HR $1.37,95 \%-\mathrm{Cl}$ 0.64-2.96) on OS or MFS (VHL mutation HR 1.16, 95\%-Cl 0.59-2.25; VHL promoter methylation HR 1.00, 95\%-Cl 0.36-2.78; and VHL changes HR 1.33, 95\%-Cl 0.67-2.64). In the multivariate analyses, neither VHL mutations nor VHL promoter methylation had a statistically significant influence on OS (VHL mutation: HR 0.39, 95\% Cl 0.14-1.11; VHL promoter methylation: HR 0.44, 95\%-Cl 0.06-3.11; VHL changes: HR 0.42, 95\%-Cl 0.141.23) or on MFS (VHL mutation: HR $0.88,95 \% \mathrm{Cl} 0.35-2.21$; VHL promoter methylation: HR 1.29, 95\%-Cl 0.33-5.06; VHL changes: HR 1.21, 95\%-Cl 0.46-3.19).

Clinicopathologic parameters did have a significant influence on OS and MFS in the multivariate analyses. Mortality and metastasis risk was increased for patients with a higher cancer stage (OS stage II cancer HR 3.48 95\%-Cl 1.14-10.63; OS stage III cancer HR 6.39 95\%-Cl 2.49-16.39; MFS stage II cancer HR 2.84 95\%-Cl 1.11-7.28; MFS stage III cancer HR $4.1295 \%-\mathrm{Cl} 1.83-9.24)$ and patients with higher tumour cell proliferation (increment of 1\%: OS HR 1.10 95\%-Cl 1.03-1.17; MFS HR 1.05, 95\%-Cl 0.99-1.12). 


\section{Overall survival}

a

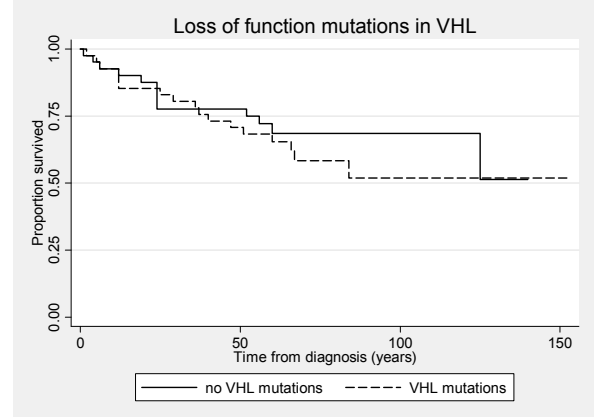

$p(\log$ rank $)=0.552$

c.

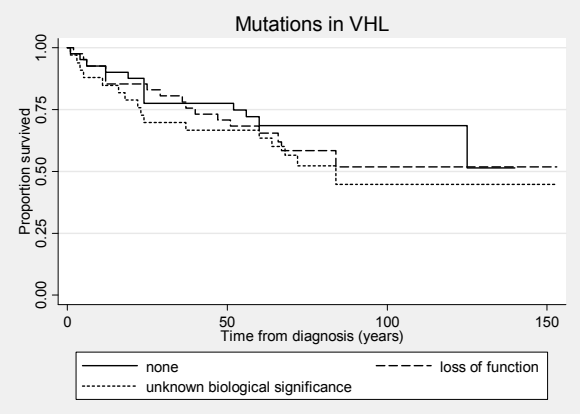

$p(\log$ rank $)=0.489$

e.

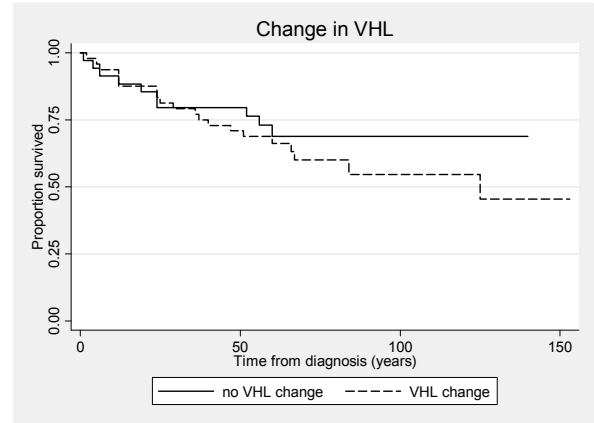

$p(\log$ rank $)=0.423$

\section{Metastatic free survival}

b.

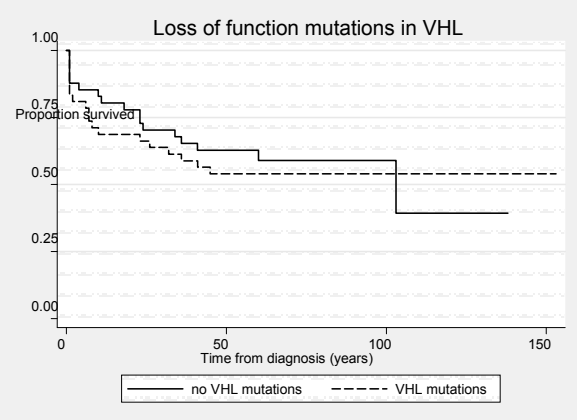

$p(\log$ rank $)=0.661$

d.

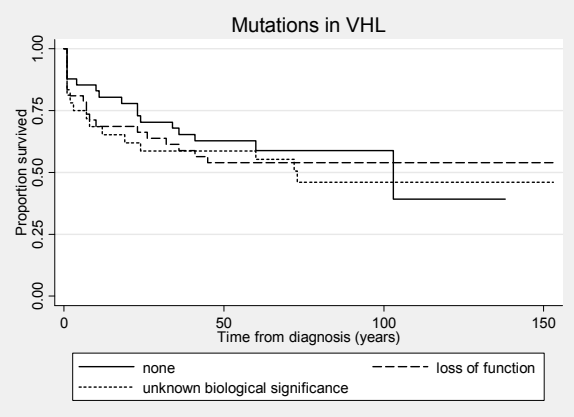

$p(\log$ rank $)=0.758$

f.

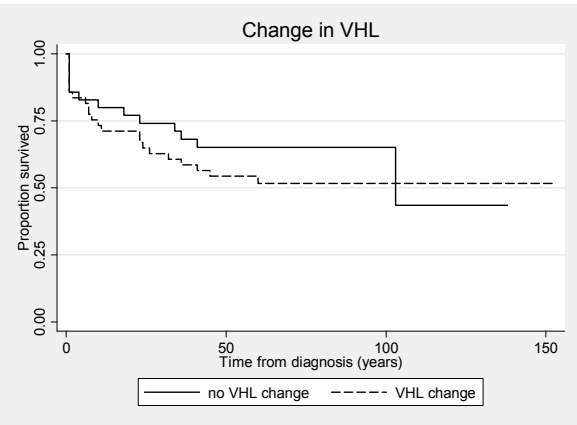

$p(\log$ rank $)=0.400$

Figure 2. Kaplan-Meier survival analyses. $\mathrm{VHL}$ change $=$ loss of function mutation and/or VHL promoter methylation 


\section{Discussion}

The aim of our study was to investigate whether VHL mutation and methylation status can explain differences in angiogenic potential between low and high grade ccRCCs. In agreement with most other studies [5,22-25] we found no significant relationship between VHL mutation (presence or type) or VHL promoter methylation and tumour parameters (grade, stage, tumour cell proliferation). In our previous study [16], we observed that high grade ccRCCs were characterized by a higher angiogenic activity than low grade ccRCCs. This difference cannot be explained by presence/absence of alterations in the VHL gene. No significant association could be found between VHL alterations and angiogenesis (ECP\%, MVD,VEGF) or HIF-1 $\alpha$ expression. Only a significant association was observed between presence of VHL change (VHL promoter methylation and/or VHL loss of function mutation) and CAIX expression. However with a p-value of 0.047 , it is not a strong significant correlation and it can be questioned whether it has any biological significance, since no significant association is found between CAIX expression and VHL promoter methylation or CAIX expression and VHL mutation status separately. CAIX plays an important role in allowing cancer cells to buffer their intracellular $\mathrm{pH}$ in hypoxic and/or in extensive glycolysis conditions [26,27], but no effect of CAIX on active angiogenesis has been shown in literature. An earlier study showed that CAIX expression is not solely regulated by HIF-1 $\alpha$ accumulation (through hypoxia and/or defective $\mathrm{VHL}$ ), resulting in binding to hypoxia response element (HRE), but also by factors binding to the juxtaposed SP1/SP3 site PR1 of the CAIX promoter, whereby induction of CAIX may be the result of a cooperation between SP1/SP3 and HIF-1 $\alpha$ transcription factors[28].

An earlier report investigated the effect of VHL mutation status on vessel density and also showed that these two features are not associated with one another [22]. Additionally, in accordance with other reports [29,30], no significant correlation was found between presence/absence or type of VHL alterations and clinical outcome. However, contrasting results about the prognostic relevance of $\mathrm{VHL}$ alterations are present in literature [31,32]. Differences between studies may be due to low samples sizes or small effect sizes [33-35], length of follow-up and postoperative treatment.

Our results suggest that regulation of angiogenesis and tumour progression of cCRCC is apparently not directly influenced by VHL alterations. The equal frequencies of VHL alterations between tumour stages 1, 2 and 3 supports the belief that VHL gene inactivation is an early event in ccRCC tumourigenesis [36,37]. Subsequent to this inactivation, additional genetic and epigenetic events might cause the diverse angiogenic and proliferative behavior during tumour progression. To that end, the strong association between tumour cell proliferation rate and endothelial cell proliferation rate, observed in our study, implies that tumour angiogenesis and tumour cell proliferation in ccRCCs may be driven by similar mechanisms.

The HIF pathway, involved in renal angiogenesis and carcinogenesis, is complex. Tumour behavior may be dictated by additional genetic defects in the hypoxia-HIF-VHL 
upstream, non-VHL oncogenic pathways on HIF activation (P13 kinase pathway $[38,39] /$ MAPK kinase pathway $[40,41]$ ), and downstream pathways (VEGF and other target genes). For instance, VEGF by itself acts through multiple cell surface receptors and signaling pathways to stimulate endothelial cell proliferation, survival, and migration [42]. By inducing other growth factor expression, VEGF stimulates a cascade of angiogenic activity [42].

Studies of HIF target genes have defined examples with putative antitumourigenic properties [43]. Also contrasting properties of HIF-1 $\alpha$ and HIF-2 $\alpha$ were observed in VHL-defective RCC cells, whereby, HIF- $2 \alpha$ yielded more protumourigenic effects than HIF-1 $\alpha$ [44], suggesting that it is the overall balance of target gene activation that determines the outcome of HIF activation.

Besides the HIF transcriptional cascade, often considered as the regulating system of tumour angiogenesis, accumulating evidence indicates that HIF-independent pathways also can control angiogenesis [45]. These mechanisms still have to be elucidated in sporadic cCRCC.

In our study, the lack of a direct association between VHL inactivation status and HIF$1 \alpha$ expression can be explained by an earlier report of Jiang et al. This study demonstrated the expression of a different number of hypoxia-inducible genes between RCC 786-0 VHL+ and 786-0 VHL' cell lines. Four genes were induced in both cell lines. These findings suggest VHL-dependent and VHL-independent mechanisms in RCC cells in response to hypoxic stress [46]. Other studies reveal that, even in the absence of VHL mutations, the frequency of HIF-1 $\alpha$ protein expression still varies from 14 to $75 \%$ $[14,47,48]$. In this case, loss of HIF-1 $\alpha$ protein was not only associated with truncated HIF-1 $\alpha$ mRNA transcripts, but also with transcriptional silencing [49]. In these HIF-1 $\alpha$ defective cell lines, the knockdown of the HIF- $2 \alpha$ gene demonstrated that HIF- $2 \alpha$ regulated the VEGF production, irrespective of the VHL gene mutation status [49].

Finally, $27 \%$ of our patient group showed no VHL mutations or VHL promoter methylation. In the literature, in approximately $25-50 \%$ of sporadic ccRCC no alterations in the VHL alleles were detected $[25,50,51]$.These findings indicate that VHL alterations may be central to the development of the vast majority of ccRCC, but not the only tumourigenic pathway. Additional inactivation of 3p12-p21 tumour suppressor genes appears to be necessary in the development of ccRCC irrespective of VHL gene inactivation $[52,53]$. Furthermore, though $3 p$ is the most frequently lost chromosome arm in CCRCC, other regions of genetic loss on other chromosomes have been identified in ccRCC [54-56]. The presence of multiple genetic losses in ccRCC is associated with a poor prognosis $[53,55]$. 
In conclusion, our data suggest that VHL alterations, though one of the key events in both hereditary and sporadic ccRCCs, may not independently influence angiogenesis, tumour proliferation or prognosis. The variable nature of cCRCC is most likely strongly determined by the complex interplay of additional downstream modifications, among which the role of epigenetic alteration of gene expression is becoming more and more acknowledged. Elucidation of these modifications will be necessary for successful targeted therapy. 


\section{References}

1 Kim, W.Y. and Kaelin, W.G. (2004) Role of VHL gene mutation in human cancer. J Clin Oncol 22, 49915004

2 Maher, E.R. (2004) Von Hippel-Lindau disease. Curr Mol Med 4, 833-842

3 Gnarra, J.R. et al. (1994) Mutations of the VHL tumour suppressor gene in renal carcinoma. Nat Genet 7, 85-90

4 Zbar, B. et al. (1996) Germline mutations in the Von Hippel-Lindau disease (VHL) gene in families from North America, Europe, and Japan. Hum Mutat 8, 348-357

5 Banks, R.E. et al. (2006) Genetic and epigenetic analysis of von Hippel-Lindau (VHL) gene alterations and relationship with clinical variables in sporadic renal cancer. Cancer Res 66, 2000-2011

6 Dulaimi, E. et al. (2004) Promoter hypermethylation profile of kidney cancer. Clin Cancer Res 10, 39723979

7 Battagli, C. et al. (2003) Promoter hypermethylation of tumor suppressor genes in urine from kidney cancer patients. Cancer Res 63, 8695-8699

8 Herman, J.G. et al. (1994) Silencing of the VHL tumor-suppressor gene by DNA methylation in renal carcinoma. Proc Natl Acad Sci U S A 91, 9700-9704

9 Ohh, M. et al. (2000) Ubiquitination of hypoxia-inducible factor requires direct binding to the betadomain of the von Hippel-Lindau protein. Nat Cell Biol 2, 423-427

10 Tanimoto, K. et al. (2000) Mechanism of regulation of the hypoxia-inducible factor-1 alpha by the von Hippel-Lindau tumor suppressor protein. Embo J 19, 4298-4309

11 Huang, L.E. et al. (1998) Regulation of hypoxia-inducible factor 1alpha is mediated by an O2-dependent degradation domain via the ubiquitin-proteasome pathway. Proc Natl Acad Sci U S A 95, 7987-7992

12 Kondo, K. and Kaelin, W.G., Jr. (2001) The von Hippel-Lindau tumor suppressor gene. Exp Cell Res 264 $117-125$

13 Carmeliet, P. et al. (1998) Role of HIF-1alpha in hypoxia-mediated apoptosis, cell proliferation and tumour angiogenesis. Nature 394, 485-490

14 Wiesener, M.S. et al. (2001) Constitutive activation of hypoxia-inducible genes related to overexpression of hypoxia-inducible factor-1alpha in clear cell renal carcinomas. Cancer Res 61, 5215-5222

15 Gnarra, J.R. et al. (1996) Post-transcriptional regulation of vascular endothelial growth factor mRNA by the product of the VHL tumor suppressor gene. Proc Natl Acad Sci U S A 93, 10589-10594

16 Baldewijns, M.M. et al. (2007) High-grade clear cell renal cell carcinoma has a higher angiogenic activity than low-grade renal cell carcinoma based on histomorphological quantification and qRT-PCR mRNA expression profile. Br J Cancer 96, 1888-1895

17 Kaelin, W.G., Jr. (2007) The von Hippel-Lindau tumor suppressor protein and clear cell renal carcinoma. Clin Cancer Res 13, 680s-684s

18 Herman, J.G. et al. (1996) Methylation-specific PCR: a novel PCR assay for methylation status of CpG islands. Proc Natl Acad Sci U S A 93, 9821-9826

19 van Engeland, M. et al. (2003) Effects of dietary folate and alcohol intake on promoter methylation in sporadic colorectal cancer: the Netherlands cohort study on diet and cancer. Cancer Res 63, 3133-3137

20 Van den Eynden, G.G. et al. (2005) Angiogenesis and hypoxia in lymph node metastases is predicted by the angiogenesis and hypoxia in the primary tumour in patients with breast cancer. $\mathrm{Br} J \mathrm{Cancer} 93$, 1128-1136

21 Foster, K. et al. (1994) Somatic mutations of the von Hippel-Lindau disease tumour suppressor gene in non-familial clear cell renal carcinoma. Hum Mol Genet 3, 2169-2173 
22 Schraml, P. et al. (2002) VHL mutations and their correlation with tumour cell proliferation, microvessel density, and patient prognosis in clear cell renal cell carcinoma. J Pathol 196, 186-193

23 Kondo, K. et al. (2002) Comprehensive mutational analysis of the VHL gene in sporadic renal cell carcinoma: relationship to clinicopathological parameters. Genes Chromosomes Cancer 34, 58-68

24 Hamano, K. et al. (2002) Biallelic inactivation of the von Hippel-Lindau tumor suppressor gene in sporadic renal cell carcinoma. $J$ Urol $167,713-717$

25 van Houwelingen, K.P. et al. (2005) Prevalence of von Hippel-Lindau gene mutations in sporadic renal cell carcinoma: results from The Netherlands cohort study. BMC Cancer 5, 57

26 Grabmaier, K. et al. (2004) Strict regulation of CAIX(G250/MN) by HIF-1alpha in clear cell renal cell carcinoma. Oncogene 23, 5624-5631

27 Semenza, G.L. (2003) Targeting HIF-1 for cancer therapy. Nat Rev Cancer 3, 721-732

28 Kaluz, S. et al. (2003) Expression of the hypoxia marker carbonic anhydrase IX is critically dependent on SP1 activity. Identification of a novel type of hypoxia-responsive enhancer. Cancer Res 63, 917-922

29 Smits, K.M. et al. (2008) Genetic and Epigenetic Alterations in the von Hippel-Lindau Gene: the Influence on Renal Cancer Prognosis. Clin Cancer Res 14, 782-787

30 Lidgren, A. et al. (2006) Hypoxia-inducible factor 1alpha expression in renal cell carcinoma analyzed by tissue microarray. Eur Urol 50, 1272-1277

31 Lidgren, A. et al. (2005) The expression of hypoxia-inducible factor 1alpha is a favorable independent prognostic factor in renal cell carcinoma. Clin Cancer Res 11, 1129-1135

32 Klatte, T. et al. (2007) Hypoxia-Inducible Factor 1 \{alpha\} in Clear Cell Renal Cell Carcinoma. Clin Cancer Res 13, 7388-7393

33 Ioannidis, J.P. (2005) Why most published research findings are false. PLoS Med 2, e124

34 Ioannidis, J.P. (2005) Molecular bias. Eur J Epidemiol 20, 739-745

35 Ioannidis, J.P. (2007) Is molecular profiling ready for use in clinical decision making? Oncologist 12, 301 311

36 Lubensky, I.A. et al. (1996) Allelic deletions of the VHL gene detected in multiple microscopic clear cell renal lesions in von Hippel-Lindau disease patients. Am J Pathol 149, 2089-2094

37 Prowse, A.H. et al. (1997) Somatic inactivation of the VHL gene in Von Hippel-Lindau disease tumors. Am J Hum Genet 60, 765-771

38 Hay, N. (2005) The Akt-mTOR tango and its relevance to cancer. Cancer Cell 8, 179-183

39 Blancher, C. et al. (2001) Effects of ras and von Hippel-Lindau (VHL) gene mutations on hypoxiainducible factor (HIF)-1alpha, HIF-2alpha, and vascular endothelial growth factor expression and their regulation by the phosphatidylinositol 3'-kinase/Akt signaling pathway. Cancer Res 61, 7349-7355

40 Oka, H. et al. (1995) Constitutive activation of mitogen-activated protein (MAP) kinases in human rena cell carcinoma. Cancer Res 55, 4182-4187

41 Huang, D. et al. (2008) Inhibition of MAPK kinase signaling pathways suppressed renal cell carcinoma growth and angiogenesis in vivo. Cancer Res 68, 81-88

42 Walsh, D.A. (2007) Pathophysiological mechanisms of angiogenesis. Adv Clin Chem 44, 187-221

43 Wykoff, C.C. et al. (2000) Identification of novel hypoxia dependent and independent target genes of the von Hippel-Lindau (VHL) tumour suppressor by mRNA differential expression profiling. Oncogene 19, 6297-6305

44 Raval, R.R. et al. (2005) Contrasting properties of hypoxia-inducible factor 1 (HIF-1) and HIF-2 in von Hippel-Lindau-associated renal cell carcinoma. Mol Cell Biol 25, 5675-5686

45 Mizukami, Y. et al. (2007) Hypoxia inducible factor-1 independent pathways in tumor angiogenesis. Clin Cancer Res 13, 5670-5674 
46 Jiang, Y. et al. (2003) Gene expression profiling in a renal cell carcinoma cell line: dissecting VHL and hypoxia-dependent pathways. Mol Cancer Res 1, 453-462

$47 \mathrm{Na}$, X. et al. (2003) Overproduction of vascular endothelial growth factor related to von Hippel-Lindau tumor suppressor gene mutations and hypoxia-inducible factor-1 alpha expression in renal cell carcinomas. J Urol 170, 588-592

48 Turner, K.J. et al. (2002) Expression of hypoxia-inducible factors in human renal cancer: relationship to angiogenesis and to the von Hippel-Lindau gene mutation. Cancer Res 62, 2957-2961

49 Shinojima, T. et al. (2007) Renal cancer cells lacking hypoxia inducible factor (HIF)-1alpha expression maintain vascular endothelial growth factor expression through HIF-2alpha. Carcinogenesis 28, 529-536

50 Weiss, R.H. and Lin, P.Y. (2006) Kidney cancer: identification of novel targets for therapy. Kidney Int 69 224-232

51 Zbar, B. et al. (2003) Studying cancer families to identify kidney cancer genes. Annu Rev Med 54, 217233

52 Morrissey, C. et al. (2001) Epigenetic inactivation of the RASSF1A 3p21.3 tumor suppressor gene in both clear cell and papillary renal cell carcinoma. Cancer Res 61, 7277-7281

53 van den Berg, A. et al. (1996) Major role for a 3p21 region and lack of involvement of the $t(3 ; 8)$ breakpoint region in the development of renal cell carcinoma suggested by loss of heterozygosity analysis. Genes Chromosomes Cancer 15, 64-72

54 Thrash-Bingham, C.A. et al. (1995) Genomic alterations and instabilities in renal cell carcinomas and their relationship to tumor pathology. Cancer Res 55, 6189-6195

55 Moch, H. et al. (1996) Genetic aberrations detected by comparative genomic hybridization are associated with clinical outcome in renal cell carcinoma. Cancer Res 56, 27-30

56 Brauch, H. et al. (1994) Genetic alterations in sporadic renal-cell carcinoma: molecular analyses of tumor suppressor gene harboring chromosomal regions 3p, 5q, and 17p. World J Urol 12, 162-168 


\section{Chapter 6}

\section{Low frequency of lymph node metastasis in clear cell renal cell carcinoma is related to low lymphangiogenic activity.}

Marcella M. Baldewijns, Tania Roskams, Vera Ballet, Gert G. Van den Eynden, Steven J. Van Laere, Ilse Van der Auwera, Evelyne Lerut, Adriaan P. De Bruïne, Victor L. Thijssen, Peter B. Vermeulen and Hein van Poppel.

BJU International 2009, Epub ahead of print 


\begin{abstract}
Objective: To assess ongoing lymphangiogenesis in renal cell carcinomas (RCC) by histomorphometry and by quantifying mRNA expression levels of lymphangiogenesisrelated factors.

Materials and methods: Using D2-40 antibody as a lymphatic marker, lymph vessel count was performed in tissue sections of 150 clear cell RCCs (cCRCC) and 61 nonneoplastic controls, by means of the Chalkley method, which in fact measures the relative lymph vessel area (LVA). Double staining with Ki67 and D2-40 was used to assess active lymphangiogenesis. In a subset of ccRCCs $(n=25)$ and non-neoplastic controls $(n=9)$ mRNA expression levels of lymphangiogenic factors were determined by real-time quantitative RT-PCR.

Results: LVA was higher in normal renal tissue compared to both intra- and peritumoural LVA $(p<0.001)$. LVA in the tumour periphery was higher than in the tumour parenchyma $(p<0.001)$. Lymphatic endothelial cell proliferation was identified in $8.2 \%$ of the control sections and was higher than the intratumoural lymphatic endothelial cell proliferation fraction (LECP\%, $2.6 \%)(p=0.02)$ and the peritumoural LECP\% (6.5\%) $(p=n . s$.$) . Compared to controls, ccRCC specimens showed higher mRNA expression$ levels of VEGF-A $(p<0.001)$ and VEGF-C $(p<0.001)$, but lower expression levels of VEGF$D(p<0.001)$ and Prox-1 $(p<0.001)$.

Conclusion: Our data demonstrate that there is only limited ongoing lymphangiogenesis present in ccRCC. Given the dual activity of several growth factors, stimulating both angiogenesis and lymphangiogenesis, our observation indirectly points to the fact that haemangiogenesis predominates in ccRCC. This finding may provide a clue to better understand why ccRCCs prefer haematogenous dissemination to lymphatic spread.
\end{abstract}




\section{Introduction}

Although haematogenous spread is the most common route of tumour dissemination in renal cell carcinoma (RCC), 7-17 \% of patients with RCC have hilar or locoregional lymph node metastases[1-3]. Tumour cells may either get access to the lymphatic system by inducing intratumoural lymphangiogenesis or by invading pre-existing lymphatics in the surrounding tissue [4-6]. Although sprouting of new lymphatic microvessels from pre-existing ones is a key process of intratumoural lymphangiogenesis, it is still unclear whether recruitment of precursor lymphatic endothelial cells participates in the formation of intratumoural lymphatic vessels $[7,8]$. The growth of new lymphatic vessels (lymphangiogenesis) is a complex process, regulated by multiple factors. In vitro and in vivo studies have demonstrated that both vascular endothelial growth factors-C and D (VEGF-C and -D) act directly on lymphatic endothelial cells via activation of vascular endothelial growth factor receptor (VEGFR-3) [9]. Indeed, overexpression of VEGF-C and -D promotes lymphatic metastasis [4,5]. Recent studies in animal models have shown that vascular endothelial growth factor-A (VEGF-A) can also stimulate lymphatic vessel growth, by inducing the release of VEGF-C and -D by activated inflammatory macrophages $[10,11]$. Furthermore, VEGFR-2 is occasionally expressed on lymphatic endothelial cells (LEC), suggesting a direct stimulatory effect of VEGF-A on lymphatic vessels through binding to VEGFR-2 [12]. Cao et al (2004) suggested that platelet derived growth factor-B (PDGF-B) may be a survival factor for newly formed lymphatics, by activating the AKT kinase, which promotes anti-apoptotic signalling. PDGF-B induced lymphatic endothelial cell differentiation and assembly of lymphatic vessels are mediated via a Prox-1 independent pathway [13]. The homeobox gene Prox-1 is an essential early transcription factor for lymphatic development and can be used as a specific marker for normal and tumour-associated lymphatic vessels[14].

The identification of molecular markers with high specificity for LECs relative to blood vessel endothelium, e.g. LYVE-1, podoplanin or D2-40, allows for the quantification of lymphangiogenesis in human tumours[15]. By measuring the fraction of proliferating LECs, based on double-immunostaining tumour sections with antibodies directed at D2-40 and Ki67, ongoing lymphangiogenesis has been described in breast adenocarcinomas, with more aggressive phenotypes having elevated LEC proliferation fraction[16].

Recent studies have investigated lymph vessel density in conventional RCC, using D240 as a lymphatic marker[17-19]. Lymph vessel density (LVD) is the net result of previous phases of tumour lymphangiogenesis and of lymphatic remodelling or regression, which implicates that the measurement of LVD is not necessarily a reflection of the ongoing tumour lymphangiogenesis. In the present study lymphangiogenesis was quantified in clear cell RCC (ccRCC), not only by evaluating LVD, but also by assessment of LEC proliferation fraction, a measure of ongoing lymphangiogenesis, and by quantifying mRNA (quantitative real-time RT-PCR) of lymphangiogenesis-related factors (Table 1). 
Table 1: Lymphangiogenesis-related factors

\begin{tabular}{|c|c|c|c|c|}
\hline Gene & Function & $\begin{array}{c}\text { Effect/ } \\
\text { presence on } \\
\text { lymph vessels }\end{array}$ & $\begin{array}{c}\text { Effect/ } \\
\text { presence on blood } \\
\text { vessels }\end{array}$ & Reference \\
\hline VEGF-A & $\begin{array}{l}\text { Growth factor } \\
\text { Induces lymphangiogenesis and } \\
\text { haemangiogenesis }\end{array}$ & + & + & {$[15,23,26]$} \\
\hline VEGF-C & $\begin{array}{l}\text { Growth factor } \\
\text { Promotes the growth of intratumoural blood } \\
\text { vessels(via activation of VEGFR-2 }{ }^{(\mathrm{a})} \text { ) and lymph } \\
\text { vessels }\end{array}$ & + & + & {$[5,15]$} \\
\hline VEGF-D & $\begin{array}{l}\text { Growth factor } \\
\text { Induces the formation of lymphatic vessel } \\
\text { within the tumour } \\
\text { Promotes haemangiogenesis via activation of } \\
\text { VEGFR-2 }\end{array}$ & + & + & {$[4,15]$} \\
\hline VEGFR-3 & $\begin{array}{l}\text { Growth factor receptor } \\
\text { Plays an important role in the development of } \\
\text { lymphatic vasculature } \\
\text { Induces proliferation of cultered LECs } \\
\text { Ligands are VEGF-C and VEGF-D }\end{array}$ & + & $+{ }^{(b)}$ & {$[9,15]$} \\
\hline PDGF-B & $\begin{array}{l}\text { Survival factor for newly formed lymphatics } \\
\text { Induces LEC differentiation and assembly of } \\
\text { lymph vessels } \\
\text { Maturation factor for newly formed tumoural } \\
\text { blood vessels }\end{array}$ & + & + & {$[13,26]$} \\
\hline PROX-1 & $\begin{array}{l}\text { Homeodomain protein involved in the } \\
\text { formation of lymphatics }\end{array}$ & + & $(+)^{(c)}$ & [14] \\
\hline
\end{tabular}

\section{Materials and methods}

\subsection{Tissue samples and patient characteristics}

Tumour samples of 150 patients with ccRCCs were obtained during radical or partial nephrectomy, from the archives of the Departments of Histopathology of the University Hospitals of Leuven and Maastricht. Tissue samples were snap-frozen or formalin fixed and paraffin embedded. None of the patients had received neo-adjuvant therapy at the time of nephrectomy. Regional lymphadenectomy was not routinely performed without clinical evidence of regional lymph node involvement. In our study group 58 of 150 patients underwent lymph node dissection, of which 10 patients showed histological proven lymph node metastases. All patients were evaluated post-operatively at regular intervals by means of physical examination, chest $x$-ray, abdominal computed tomography or ultrasound and blood chemistry. The median follow-up time was 64 months (range 1-153 months). Five patients showed lymph node metastases at the time of recurrence. Specific clinical-pathological data of the study population are summarized in Table 2. The control population consisted of 61 samples of tumour-free tissue, collected in the opposite tumour-free kidney pole of the nephrectomy specimens. 
Low frequency of lymph node metastasis

\begin{tabular}{|c|c|c|}
\hline & $\begin{array}{l}\text { Immunohistochemistry } \\
\text { Clear cell RCC }\end{array}$ & $\begin{array}{l}\text { Real-time RT-PCR } \\
\text { Clear cell RCC }\end{array}$ \\
\hline Number of patients & 150 & 25 \\
\hline Gender $M / F$ & $99 / 51$ (66/34\%) & $13(52 \%)$ \\
\hline Age $^{a}$ (years) & $60.6(22-82)$ & $63.1(32-78)$ \\
\hline Tumour diameter $^{\mathrm{a}}(\mathrm{cm})$ & $6.36(0.7-20.0)$ & $6.34(3.0-13.0)$ \\
\hline \multicolumn{3}{|l|}{ Fuhrman grade } \\
\hline 1 & $15(10 \%)$ & $4(16 \%)$ \\
\hline 2 & 72 (48\%) & $15(60 \%)$ \\
\hline 3 & $46(30.7 \%)$ & $5(20 \%)$ \\
\hline 4 & $17(11.3 \%)$ & $1(4 \%)$ \\
\hline \multicolumn{3}{|l|}{ Tumour stage $^{b}$} \\
\hline I & $82(54.7 \%)$ & $14(56 \%)$ \\
\hline II & $24(16 \%)$ & $5(20 \%)$ \\
\hline III & $42(28 \%)$ & $6(24 \%)$ \\
\hline IV & $2(1.3 \%)$ & $0(0 \%)$ \\
\hline \multicolumn{3}{|l|}{ Lymph node status } \\
\hline Negative/positive & $135 / 15(10 \%)$ & $22 / 3(12 \%)$ \\
\hline
\end{tabular}

\subsection{Immunohistochemical staining}

To assess lymph vessel density and to analyse proliferating LECs, double immunostaining for D2-40 and Ki-67 was performed by a Dako Autostainer (Dako), as described before [16]. Serial 4 micron thick sections were cut from formalin fixed paraffinembedded tumour tissue $(n=195)$ and from normal renal tissue paraffin blocks $(n=61)$. After dewaxing in toluene and rehydration through sequential changes of alcohol and distilled water, sections were first incubated with the Ki-67 (clone MIB-1, dilution 1/150, Dako, Glöstrup, Denmark,) primary antibody which was visualized with Envision + dual link system (Dako). A second primary antibody against podoplanin (clone D2-40, dilution 1/20, Dako) was then applied and visualized with Envision G/2 system/AP (Dako).

\subsection{Assessment of lymph vessel area and lymphatic endothelial cell proliferation}

Immunostained tumour and normal renal sections were scanned at low magnification $(100 x)$. For quantification, regions with the highest lymphatic density (hot spots) were chosen both in tumoural and peritumoural tissue. Intratumoural lymph vessels were defined as D2-40 positive vessels that were in close contact with tumour cells or located in fibrous tissue surrounding tumour cells. Peritumoural lymph vessels were defined as D2-40 positive vessels in the fibrous capsule, at the interface of tumour and adjacent kidney or pre-existing normal renal tissue at a maximal distance of $2 \mathrm{~mm}$ from the tumour periphery. The presence of a lumen was not necessary to identify a lymph vessel in order not to exclude small sprouting lymph vessels. At higher magnification (200x), quantification of lymph vessels was performed according to the Chalkley point overlap morphometric technique [20]. This method involves the use of an eyepiece graticule containing 25 randomly positioned dots, which is rotated so that the 
maximum number of points is on or within the vessels of the vascular "hot spot". Thus, the Chalkley count is the number of grid points that hit stained lymph vessels. It is a relative area estimate rather than a true vessel count. In every section of normal or renal tumour tissue, in three hot spots (areas of highest lymph vessel density), the eyepiece graticule was rotated so that the maximum number of points was on or within the vessels of every vascular hot spot. The mean value of the counts in the three hot spots was considered as the Chalkley count of that specimen and taken for further analysis.

The fraction of proliferating lymphatic endothelial cells (LECP\%) was calculated in each hot spot as the number of LECs with Ki-67 stained nuclei per 100 lymphatic endothelial cells $\times 100$ (\%). Furthermore, intra- and peritumoural lymph vessels were scanned for the presence of tumour emboli. All these assessments were made by two independent observers (inter-observer variability $<5 \%$ ).

\subsection{RNA isolation, CDNA synthesis and qPCR analysis}

Quantitative real-time RT-PCR was performed to determine mRNA expression levels in 25 clear cell RCC (ccRCC) tissues and 9 normal renal tissues. For this, total RNA was isolated using the RNeasy mini kit (QIAgen) from 10 cryosections ( $20 \mu \mathrm{m}$ thick) of frozen tissue samples. On column DNAse treatment was performed to remove any genomic DNA contaminations. Concentration and quality of the RNA was analysed on the NanoDrop ND-1000 (NanoDrop Technologies, Inc., Wilmington, USA) and by agarose gel electrophoresis, respectively. CDNA synthesis was performed with the iscript CDNA synthesis kit (Biorad, Hercules, CA) on $100 \mathrm{ng}$ RNA according to the suppliers protocol. Following CDNA synthesis, nuclease free water was added to a final volume of $50 \mu \mathrm{l}$ and samples were stored at $-20^{\circ} \mathrm{C}$. Quantitative real-time RT-PCR was performed on the iCycler (Biorad) with $2 \mu \mathrm{l} \mathrm{CDNA}$ and the iQ SYBR green Supermix (Biorad) as described previously[21]. Primers were synthesized by Eurogentec and targeted against VEGF-A, VEGF-B, VEGF-C, VEGF-D, VEGFR-3, PDGF-B and Prox-1. Primers targeted against cyclophilin $A$, beta-actin and $18 S$ rRNA were used as reference genes for normalization of the $\mathrm{Ct}$ values. Relative gene expressions were presented as $2^{-\mathrm{ACt}}$ [22].

\section{Statistical analysis}

Statistical analysis was performed with the SPSS 12.0 software package. A p-value < 0.05 was considered statistically significant. Normality was tested with a KolmogorovSmirnov test assuming normality of data if $p \geq 0.2$. If continuous data (e.g. RT-PCR expression, LECP\%) were normally distributed, correlations were analyzed with Pearson correlation statistics, if not with Spearman correlation statistics. In case of normal distribution in all subgroups, equality of means was tested with a student T-test, if data 
were not normally distributed equality of medians was tested with a Mann-Whitney U test.

\section{Results}

\subsection{Immunohistochemical quantification of lymph vessels}

The D2-40 antibody showed strong immunoreactivity with lymph vessel endothelium, both in the tumour (center and periphery) as well as the surrounding normal renal tissue. Of the renal tumours, $36 \%$ revealed D2-40 positive intratumoural lymph vessels and $83 \%$ D2-40 positive peritumoural lymph vessels. In $98 \%$ of the normal renal tissue samples, D2-40 positive lymph vessels were present. In normal renal tissue sections lymph vessels were abundant around intrarenal blood vessels. In the interstitium only a few lymph vessels were present around tubules and glomeruli, except in case of interstitial inflammation when clusters of lymph vessels were seen (Figure 1).

The intra- and peritumoural lymph vessels were often irregularly shaped and collapsed. No intralymphatic tumour emboli could be found. Hot spots of intratumoural lymph vessels were especially seen in inflammatory response areas within the substance of the tumour and in the neighbourhood of blood vessels (Figure 1). Hot spots of peritumoural lymph vessels were in $95 \%$ of all cases present in the peritumoural fibrous capsule (Figure 1).

Table 3 shows the results of the lymphangiogenesis parameters in normal renal tissue and clear cell RCC. The Chalkley count of the control non-neoplastic renal tissue was significantly higher than the intra- or peritumoural LVA $(p<0.001)$. Peritumoural LVA appeared to be significantly higher than the intratumoural LVA $(p<0.001)$. Neither intratumoural LVA nor peritumoural LVA correlated significantly with clinicopathological variables (lymph node status, Fuhrman grade and tumour stage) (data not shown). 

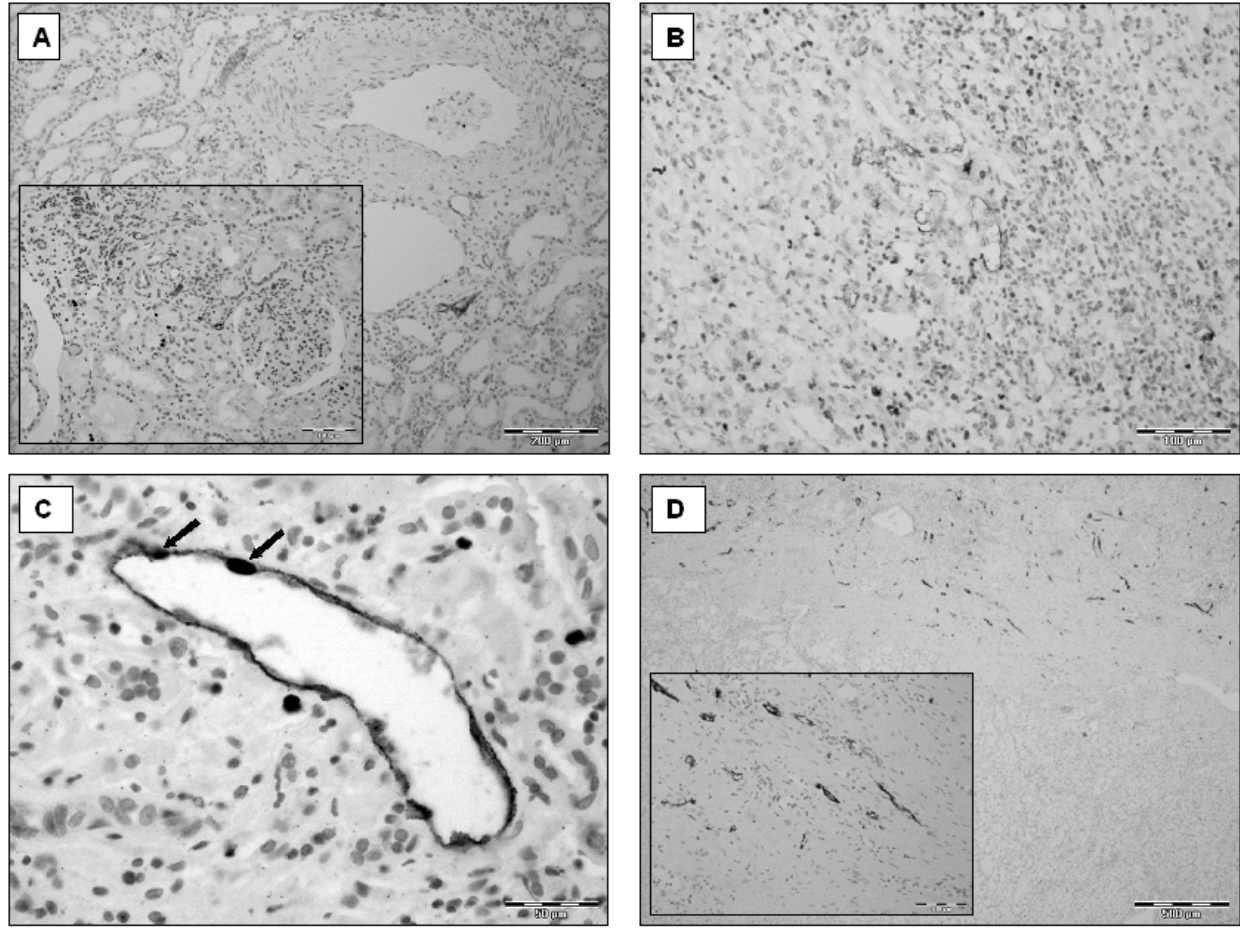

Figure 1: Overview of the D2-40/Ki-67 double immunostainings. A: non-neoplastic renal tissue with the presence of lymph vessels (red) around intrarenal veins and arteries. Inset illustrates lymphatic capillaries in the neighbourhood of a glomerulus, in the background of an inflammatory interstitium of the tumor-free renal tissue. B: Intratumoural lymph vessels were observed especially in inflammatory areas. C: Black arrows show proliferating Ki-67 positive (brown nucleus) endothelial cells. D: Hot spots of peritumoural lymph vessels were present in the peritumoural fibrous capsule. Lymphatic capillaries were often irregularly shaped and collapsed (inset). For full colour picture see page 166.

Table 3: Immunohistochemical quantification of lymph vessels (LVA) and proliferating lymph endothelial cells (LECP\%) in normal renal tissue and clear cell RCC.

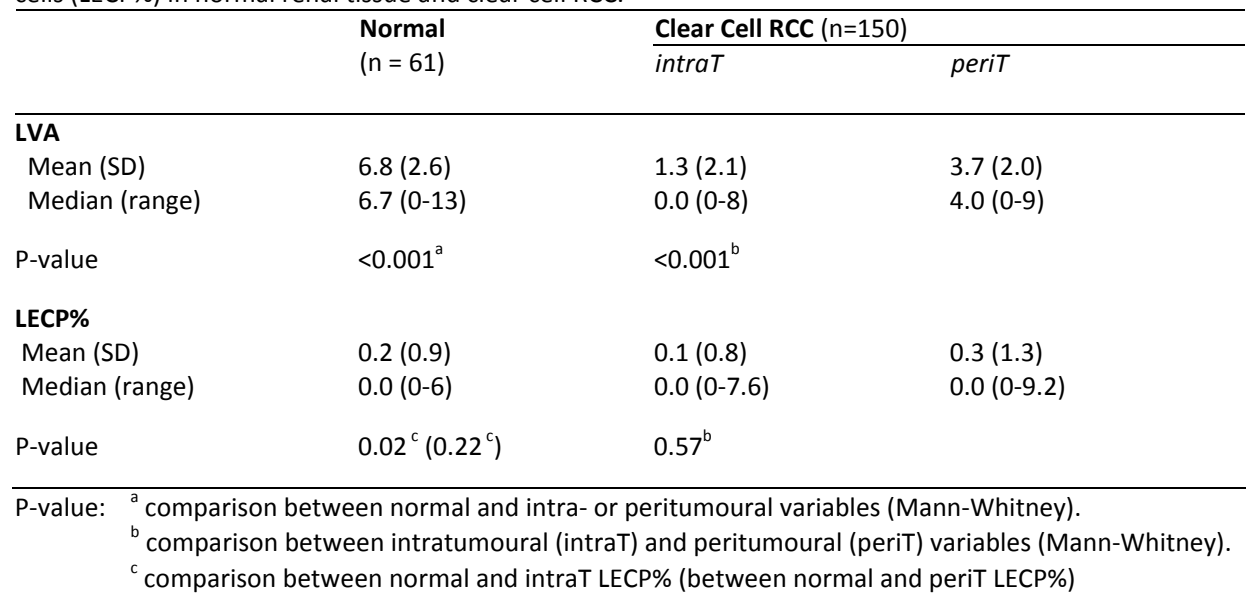




\subsection{Lymphatic endothelial cell proliferation (LECP\%)}

Lymphatic endothelial cell proliferation (LECP\%) was identified in $8.2 \%$ of the normal renal tissue sections. Proliferating LECs were seen in lymph vessels surrounding intrarenal arteries and veins as well as in lymphatic capillaries located in an inflammatory interstitium. The fraction of proliferating LECs was significant lower than in intratumoural lymph vessels $(p=0.02)$ (Table 3$)$. There were no significant differences between LECP\% of normal renal tissue and of peritumoural lymph vessels. In only $2 \%$ of the cCRCCs intratumoral lymph vessels revealed proliferating LECs and in $6 \%$ of the renal tumours proliferating LECs were present in the peritumoural lymph vessels. There were no significant differences between LECP\% of intra- and peritumoural lymph vessels.

A significant association between peritumoural LECP\% and tumour stage was found $(p=0.04)$, with a mean peritumoural LECP\% of 0.00214 in stage 1-2 RCCs and 0.0049 in stage 3-4 RCCs. However, after making a scatterplot (not shown), no real visual differences were seen comparing both tumour stage groups, presuming that this finding has no biological significance. No further significant correlations were present between LECP\% and lymph node status, Fuhrman grade or tumour stage (data not shown).

\subsection{Differential expression of genes related to lymphangiogenesis}

In ccRCC a significant overexpression of VEGF-A ( $p<0.001)$ and VEGF-C $(p=0.004)$ was detected compared with normal renal tissue (Figure 2$)$. In contrast, VEGF-D $(p<0.001)$ and Prox-1 $(p<0.001)$ expression was significantly lower. There were no significant differences of PDGF-B or VEGFR-3 expression between normal renal tissue and renal tumour. VEGF-C expression in RCC was significantly lower than VEGF-A expression $(p<0.001)$. Except for a lower PDGF-B and VEGFR-3 expression in Fuhrman grade 3-4 $R C C s$, compared with Fuhrman grade $1-2$ RCCs $(p=0.007$ and $p=0.03$, respectively), no further significant correlations were found between lymphangiogenic factor expression and clinicopathological para-meters (data not shown). No association was present between the expression of lymphangiogenesis-related factors and LVA (intra- and peritumoural) or LECP\% (intra- and peri-tumoural). 


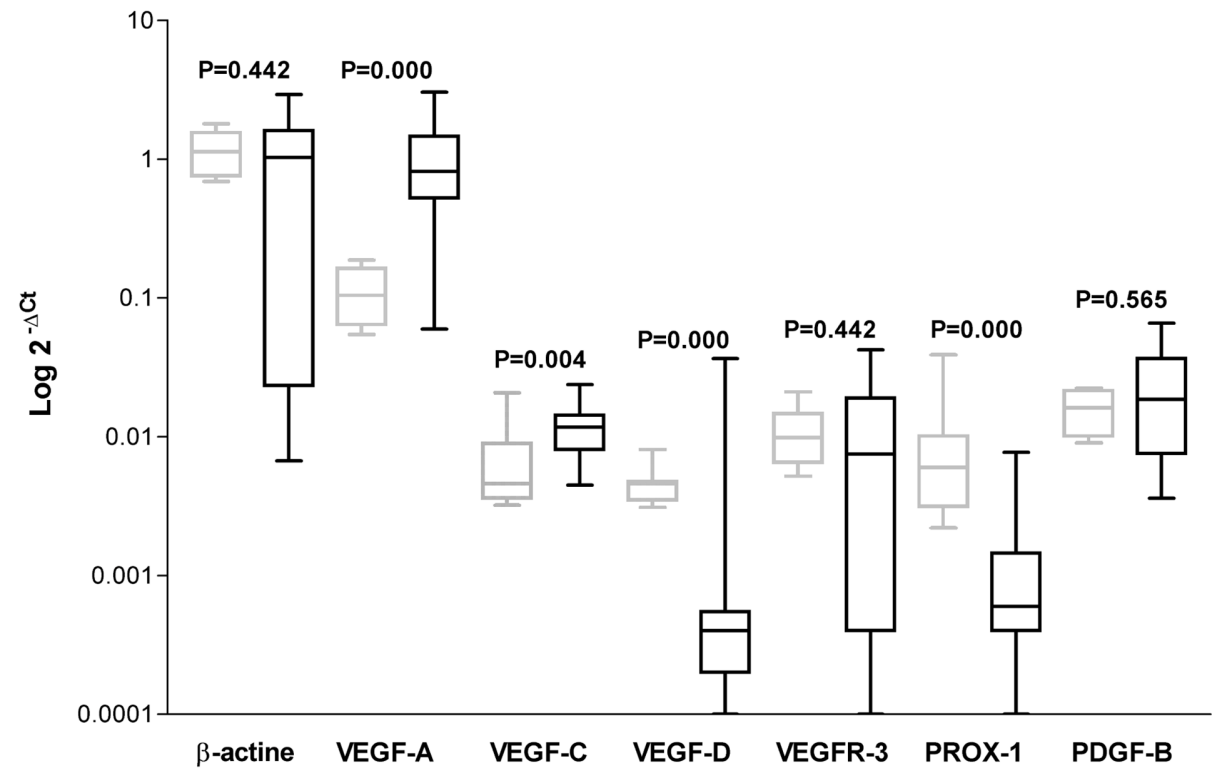

Figure 2: Box plot of real-time quantitative RT-PCR results of lymphangiogenesis related genes in nonneoplastic renal tissue (light-coloured bar) and renal tumour (dark grey bar).

\section{Discussion}

Recent studies have revealed that tumour lymphangiogenesis promotes lymph node metastasis in an experimental cancer metastasis model[4,5]. Since lymph node metastases are diagnosed in 7-17 \% of patients with RCC[3], the aim of our study was to investigate whether active lymphangiogenesis occurs in ccRCCs.

The main conclusion of the histomorphometrical part of this study is that in ccRCC there is very limited ongoing lymphangiogenesis. In only $2 \%$ of the ccRCC proliferating LECs could be demonstrated, resulting in a median proliferation fraction of 0 . With identical methodology, we have shown significantly higher LECP\% in breast carcinomas[16], with median values, depending on tumour biotype and area, ranging from 1.8 $\%$ to $8.6 \%$. This tumour type is known to metastasize to regional lymph nodes.

Furthermore, intratumoural LVA in CCRCC was significantly lower than in the normal renal tissue and peritumoural tissue, which was defined as the fibrous capsule surrounding the tumour and which actually corresponds with compressed normal renal tissue, since most of ccRCCs are expansively growing tumours.

The absence of significant lymphangiogenesis in RCC was further supported by the mRNA expression level study in which VEGF-D expression and Prox-1 expression were lower in RCC compared to control tissue. No significant differences were found for VEGF-R3 and PDGF-B. VEGF-A levels were much higher than VEGF-C levels in RCC, and 
VEGF-A has been shown to be positively related with blood vessel density in RCC[23]. Moreover, no significant correlations between the expression levels of lymphangiogenesis related factors and LVA or LECP\% could be demonstrated. The lack of an association between LVA and the presence/absence of lymph node metastases can be explained by the small number of intratumoural lymph vessels and by the fact that these vessels may not be functional[24]. In earlier studies, performed on ccRCCs, an association with lymph node involvement was demonstrated only in case of lymphatic invasion in the periphery or outside the tumour[17,25]. Clear cell RCCs are highly angiogenic tumours[26] and grow by compressing the renal parenchyma forming a new stromal tissue component with high density of proliferating capillary blood vessels. Normal tubuli or glomeruli are rarely present in the tumour parenchyma. Angiogenesis in ccRCC is to a large extent driven by VHL protein dysfunction creating a hypoxiamimicking signal that leads to upregulation of VEGF-A and other angiogenic growth factors[27]. These growth factors exhibit overlapping haemangiogenic and lymphangiogenic activity. Exposure of blood vessels and lymphatic vessels to these growth factors leads to simultaneous stimulation of angiogenesis and lymphangiogenesis[28]. Given the dual activity of several angiogenic factors and receptors, lymphangiogenic activity in ccRCC might be expected. However, our results indicate that there is no significant lymphangiogenesis in ccRCCs, presuming a preferential haemangiogenic response to these growth factors. The molecular mechanisms underlying the differential effects of the same growth factors on blood- and lymph vessels is currently unknown. Earlier observations indicated that lymphangiogenesis can occur independently from haemangiogenesis and that haemangiogenic/lymphangiogenic factors may, depending on where they are expressed in a tissue, differentially induce haemangiogenesis or lymphangiogenesis[28]. This may explain differential angiogenic and/or lymphangiogenic response in different tumour subtypes.

Regarding the significantly lower VEGF-D and Prox-1 expression in RCC compared with normal renal tissue, one could even postulate an inhibition of lymphangiogenesis within clear cell RCC. It is described that certain tumours, such as fibrosarcomas appear to produce circulating lymphangiogenesis inhibitors[28].

The presence of proliferating LECs in non-neoplastic renal tissue can be partly explained by the fact that proliferating lymph vessels were often seen in inflammatory regions. Stuht et al. found a close association between lymphangiogenesis and the presence of inflammatory infiltrates in transplanted kidney [29]. It was suggested that cellular infiltrates and their inflammatory potential may be a cause of lymphangiogenesis. VEGF-A recruitment of macrophages plays a important role in inducing lymphangiogenesis [11]. In addition to the paracrine release of VEGF-C and VEGF-D by activated inflammatory macrophages, in vitro experiments have demonstrated that CD11b-positive macrophages alone were capable of forming tubular like structures, which expressed markers of LEC [30]. 
In summary, the results of this study strongly suggest that there is only limited intratumoural lymphangiogenesis in clear cell RCC, which is not related to Fuhrman grade or tumour- and lymph node stage. These findings, together with the results of our earlier study[26], suggest that during renal tumourigenesis, haemangiogenesis rather than lymphangiogenesis occurs. This may explain why haematogenous spread is indeed the most common route of tumour dissemination in RCC. 


\section{References}

1 Bonsib, S.M. (2004) The renal sinus is the principal invasive pathway: a prospective study of 100 renal cell carcinomas. Am J Surg Pathol 28, 1594-1600

2 Minervini, A. et al. (2001) Regional lymph node dissection in the treatment of renal cell carcinoma: is it useful in patients with no suspected adenopathy before or during surgery? BJU Int 88, 169-172

3 Terrone, C. et al. (2006) Reassessing the current TNM lymph node staging for renal cell carcinoma. Eur Urol 49, 324-331

4 Stacker, S.A. et al. (2001) VEGF-D promotes the metastatic spread of tumor cells via the lymphatics. Nat Med 7, 186-191

5 Skobe, M. et al. (2001) Induction of tumor lymphangiogenesis by VEGF-C promotes breast cancer metastasis. Nat Med 7, 192-198

6 Padera, T.P. et al. (2002) Lymphatic metastasis in the absence of functional intratumor lymphatics. Science 296, 1883-1886

7 Salven, P. et al. (2003) VEGFR-3 and CD133 identify a population of CD34+ lymphatic/vascular endothelial precursor cells. Blood 101, 168-172

8 He, Y. et al. (2004) Preexisting lymphatic endothelium but not endothelial progenitor cells are essential for tumor lymphangiogenesis and lymphatic metastasis. Cancer Res 64, 3737-3740

9 Jussila, L. and Alitalo, K. (2002) Vascular growth factors and lymphangiogenesis. Physiol Rev 82, 673-700

10 Nagy, J.A. et al. (2002) Vascular permeability factor/vascular endothelial growth factor induces lymphangiogenesis as well as angiogenesis. J Exp Med 196, 1497-1506

11 Cursiefen, C. et al. (2004) VEGF-A stimulates lymphangiogenesis and hemangiogenesis in inflammatory neovascularization via macrophage recruitment. J Clin Invest 113, 1040-1050

12 Partanen, T.A. et al. (1999) Endothelial growth factor receptors in human fetal heart. Circulation 100, 583-586

13 Cao, R. et al. (2004) PDGF-BB induces intratumoral lymphangiogenesis and promotes lymphatic metastasis. Cancer Cell 6, 333-345

14 Hong, Y.K. and Detmar, M. (2003) Prox1, master regulator of the lymphatic vasculature phenotype. Cell Tissue Res 314, 85-92

15 Van der Auwera, l. et al. (2006) First international consensus on the methodology of lymphangiogenesis quantification in solid human tumours. Br J Cancer 95, 1611-1625

16 Van der Auwera, I. et al. (2005) Tumor lymphangiogenesis in inflammatory breast carcinoma: a histomorphometric study. Clin Cancer Res 11, 7637-7642

17 Ishikawa, Y. et al. (2007) Significance of lymphatic invasion and proliferation on regional lymph node metastasis in renal cell carcinoma. Am J Clin Pathol 128, 198-207

18 Horiguchi, A. et al. (2008) Intratumoral Lymphatics and Lymphatic Invasion Are Associated with Tumor Aggressiveness and Poor Prognosis in Renal Cell Carcinoma. Urology

19 Iwata, T. et al. (2008) Lymphangiogenesis and angiogenesis in conventional renal cell carcinoma: association with vascular endothelial growth factors A to D immunohistochemistry. Urology 71, 749-754

20 Fox, S.B. et al. (1995) Quantitation and prognostic value of breast cancer angiogenesis: comparison of microvessel density, Chalkley count, and computer image analysis. J Pathol 177, 275-283

21 Thijssen, V.L. et al. (2004) Angiogenesis gene expression profiling in xenograft models to study cellular interactions. Exp Cell Res 299, 286-293

22 Livak, K.J. and Schmittgen, T.D. (2001) Analysis of relative gene expression data using real-time quantitative PCR and the 2(-Delta Delta C(T)) Method. Methods 25, 402-408 
23 Tomisawa, M. et al. (1999) Expression pattern of vascular endothelial growth factor isoform is closely correlated with tumour stage and vascularisation in renal cell carcinoma. Eur J Cancer 35, 133-137

24 Padera, T.P. et al. (2004) Pathology: cancer cells compress intratumour vessels. Nature 427, 695

25 Bonsib, S.M. (2006) Renal lymphatics, and lymphatic involvement in sinus vein invasive (pT3b) clear cell renal cell carcinoma: a study of 40 cases. Mod Pathol 19, 746-753

26 Baldewijns, M.M. et al. (2007) High-grade clear cell renal cell carcinoma has a higher angiogenic activity than low-grade renal cell carcinoma based on histomorphological quantification and qRT-PCR mRNA expression profile. Br J Cancer 96, 1888-1895

27 Turner, K.J. et al. (2002) Expression of hypoxia-inducible factors in human renal cancer: relationship to angiogenesis and to the von Hippel-Lindau gene mutation. Cancer Res 62, 2957-2961

28 Cao, Y. (2005) Opinion: emerging mechanisms of tumour lymphangiogenesis and lymphatic metastasis. Nat Rev Cancer 5, 735-743

29 Stuht, S. et al. (2007) Lymphatic neoangiogenesis in human renal allografts: results from sequential protocol biopsies. Am J Transplant 7, 377-384

30 Maruyama, K. et al. (2005) Inflammation-induced lymphangiogenesis in the cornea arises from CD11bpositive macrophages. J Clin Invest 115, 2363-2372 


\section{Chapter 7}

\section{Genetics and epigenetics of renal cell cancer}

Marcella M.L. Baldewijns, Iris J.H. van Vlodrop, Leo J. Schouten, Patricia M.M.B.

Soetekouw, Adriaan P. de Bruïne and Manon van Engeland

Biochim Biophys Acta. 2008 Apr;1785(2):133-55. Review (second part). 


\begin{abstract}
Renal cell carcinoma (RCC) is not a single disease, but comprises a group of tumours of renal epithelial origin, each with a different histology, displaying a different clinical course and caused by different genetic alterations. Since cure rates are inversely associated with stage and response to the available treatment regimes is limited to a subgroup of patients, diagnostic methods facilitating early detection and new therapeutic modalities are necessary. Increased knowledge of the underlying pathophysiology of RCC has resulted in the identification of genetic alterations involved in renal cell cancer carcinogenesis. Promising agents to target these pathways, especially the angiogenesis pathway, are being developed, some of which are already standard of care. In addition to genetics, knowledge on epigenetics in the process of renal tumourigenesis has been significantly increased in the last decades. Epigenetics will play an increasing role in the development of new therapeutic modalities and may deliver new prognostic and early diagnostic markers.

In this review we discuss the background of RCC and the clinical applications of RCC genetics and epigenetics.
\end{abstract}




\section{Genetics of RCC}

During the past two decades, genetic and clinical studies have shown that RCCs are not only heterogeneous in their histology and clinical behaviour, but also in their genetic alterations. The identification of families with distinct histological subtypes of RCC has permitted a better understanding of the molecular mechanism of carcinogenesis of the different subtypes, and for each subtype one or more important mutations have been described.

Clear cell, papillary, and chromophobe RCC cover the majority of all renal cancers and are mostly investigated, therefore (genetic) knowledge of the other subtypes, with a very low prevalence, is limited. The genetics of the three most common subtypes will be discussed in this review.

\subsection{Clear cell renal cell carcinoma - von Hippel-Lindau (VHL) tumour suppressor gene}

The most and probably best studied adult renal neoplasm is ccRCC, and particularly the inherited form, related to the von Hippel-Lindau (VHL) syndrome. RCC in the heritable $V H L$-syndrome is uniformly of the clear cell subtype[1,2]. In 1988 the $V H L$ gene was mapped to the short arm of chromosome 3 by linkage analysis[3] and in 1993 the gene was identified as a result of positional cloning strategies performed in VHL kindreds[4]. Heritable ccRCC is caused by a germline mutation of one allele $(80 \%$ is inherited of one of the parents and $20 \%$ is a 'de novo' mutation) and an acquired mutation of the second allele. Germline $V H L$ mutations are identified in nearly $100 \%$ of $V H L$-families[5]. The VHL gene has been found to have characteristics of a tumour suppressor gene (TSG), in which >150 different germline mutations linked to VHL disease have been identified since 1993[1,2,6,7]. VHL mutations are extremely heterogeneous and are distributed throughout the coding sequence, except that mutations are rarely observed within the first 50 codons[2,8].

In the majority (50\%-75\%)[8-10] of sporadic ccRCCs, VHL inactivation of both alleles have been observed[11], whereby loss of heterozygosity (LOH) of a VHL allele is detected in almost all (90\%) sporadic clear cell tumours[12]. Inactivation can be a result of somatic mutations, deletions, LOH or epigenetic inactivation such as promoter DNA methylation (see also chapter 3). Although mutations in the $\mathrm{VHL}$ gene are mostly restricted to ccRCC[13], van Houwelingen et al. reported mutations in the other sporadic RCC subtypes, although in much smaller proportions[8].

Heritable and sporadic ccRCC often show two inactivated or silenced $V H L$ alleles, which indicates that the loss of function of the $V H L$ gene is an important event in the pathogenesis of $\operatorname{ccRCC}[10]$.

The VHL protein ( $p \mathrm{VHL}$ ) is the product of the VHL gene. Cultured cells produce two proteins, a protein consisting of 213 amino acid residues with a molecular weight of 30 $\mathrm{kDa}\left(\mathrm{pVHL} \mathrm{L}_{30}\right)$ and a variant which corresponds to $\mathrm{pVHL}$ residues $54-213$ of $\mathrm{pVHL}_{30}$. The 
latter migrates with a molecular weight of $18 / 19 \mathrm{kDa}\left(\mathrm{pVHL}_{18 / 19}\right)$ and is most likely generated by internal transcription initiation from the second methionine start site within the VHL open reading frame (Met-54)[14-16].

Insights into the function of $\mathrm{pVHL}$ have come from studying the effects of reintroducing the wildtype $V H L$ gene into cultures of cells which lack functional pVHL[7,13,17]. In these experiments, several aspects of cellular behaviour, related to tumour suppressor function, like growth inhibiting, cell cycle arrest, and increased susceptibility to apoptosis, were restored upon reintroduction of the wildtype $V H L$ gene[7,13].

Probably the best known alteration in VHL defective cells is the failure to (poly)ubiquitinate and degrade hypoxia inducible factor $\alpha$ (HIF- $\alpha$ ) in the presence of oxygen. HIF- $1 \alpha$ and HIF- $2 \alpha$, which are unstable but continuously transcribed and translated, are key mediators in the complex cellular and systemic oxygen adaptation cascade triggered by hypoxia[18]. Under normoxic conditions, HIF- $\alpha$ subunits become hydroxylated, enabling binding and ubiquitination by $\mathrm{pVHL}$. Under hypoxic conditions, HIF- $\alpha$ subunits remain non-hydroxylated and are able to complex with stable $\beta$ subunits. HIF $\alpha-\beta$ heterodimers bind DNA at hypoxia responsive elements (HREs) and transcriptionally activate genes involved in acute and chronic adaptation to hypoxia, normally inhibited by $\mathrm{pVHL}[18,19]$. The proteins regulated by hypoxia-inducible genes are involved in angiogenesis (vascular endothelial growth factor (VEGF)), cell growth (transforming growth factor $\alpha$ (TGF- $\alpha$ ) and platelet derived growth factor (PDGF)), glucose uptake (glucose transporter GLUT-1), and acid-base balance (carbonic anhydrase IX (CAIX)). Thus, like in hypoxic conditions, absence of (functional) pVHL leads to accumulation of HIF- $\alpha$ subunits, forming heterodimers with HIF- $\beta$, resulting in overexpression of the above mentioned proteins, which creates a microenvironment favourable for tumour proliferation[13,18]. In addition, the above mentioned proteins act on the neighbouring vasculature to promote tumour angiogenesis[13].

Both $\mathrm{VHL}$ gene products, $\mathrm{pVHL}_{30}$ and $\mathrm{pVHL}_{18 / 19}$, can inhibit the production of the hypoxia-inducible proteins when reintroduced into renal carcinoma cells that lack the wild-type $V H L$ allele. Therefore, mutations 5 ' of codon 54 may not lead to a functionally inactive protein, however mutations in the first 50 codons are rarely observed[8]. It has been observed early that RCCs are highly angiogenic tumours with aberrant expression of HIF proteins, however, Maxwell et al. were the first to report (1999) the relationship between $V H L$ disease and HIF- $\alpha$ in RCC[20]. They reported that cells lacking $\mathrm{PVHL}$ are unable to degrade HIF- $\alpha$ under both normoxic and hypoxic conditions, thus cells deficient in pVHL behave as being hypoxic (pseudohypoxia), even in normoxic conditions, due to continuous HIF- $\alpha$ activation $[9,13,20]$.

Although the HIF- $\alpha$ accumulation is important for pathogenesis or progression, it is not per se sufficient to induce tumourigenesis of ccRCC. In VHL disease only a subgroup of VHL mutation carriers develops RCC.[13,21] In addition, in approximately $25 \%-50 \%$ of sporadic ccRCC no alteration in the VHL gene was detected[8-10]. This suggests involvement of other genes that may also predispose to tumourigenesis of ccRCC, possi- 
bly affecting the same signalling pathway as $V H L$, or other mechanisms to inactivate VHL.

\subsection{Papillary renal cell carcinoma-MET proto-oncogene}

The second most prevalent kidney cancer is pRCC, which was first reported by Zbar et al in 1994[22], who observed kidney cancer in a family setting, which differed in several aspects from familial ccRCC. All tumours displayed a papillary histology without evidence of VHL mutations. A novel inherited form of kidney cancer was detected, namely hereditary papillary renal cell carcinoma[22,23]. Genetic studies in kindreds of familial pRCC led to the identification of the MET proto-oncogene gene, located on the long arm of chromosome 7[24]. Patients with (germline) mutations in the MET gene develop so-called pRCC type 1 tumours. Activating (gain of function) germline mutations in the tyrosine kinase domain of MET were found in familial pRCC patients[10,25]. MET encodes a transmembrane receptor tyrosine kinase with hepatocyte growth factor (HGF) as its ligand[6,23]. Activation of MET by HGF activates the tyrosine kinase activity, which initiates several signal transduction cascades, resulting in multiple cellular processes like mitogenesis and migration[23].

Only a small percentage $(\sim 5 \%)[24,25]$ of sporadic pRCC cases have MET mutations $[10,24,25]$. Cytogenetically, trisomy of chromosome 7 is observed in $~ 75 \%$ of the sporadic papillary cases[13]. Since this chromosome is harbouring the MET gene, the gene dose is increased. Pathogenesis of pRCC is different in the heritable and sporadic form, and as in cCRCC there may be a role for other genes in the tumourigenesis.

\subsection{Papillary renal cell carcinoma - fumarate hydratase (FH) tumour suppressor gene}

In addition to the MET gene, a second gene involved in PRCC has been identified; the fumarate hydratase $(\mathrm{FH})$ gene, located on chromosome 1q42.3-43. This gene encodes $\mathrm{FH}$, an enzyme part of the tricarboxylic acid (Krebs) cycle, which catalyzes the conversion of fumarate to malate. Little is known on the etiology and pathogenesis of these cancers: the gene is thought to act as a tumour suppressor gene[1,26] as loss of the wildtype allele is found frequently in pRCC. Tomlinson et al. evaluated seven familial pRCCs of which five showed allelic loss, one family had a 2-bp deletion and one family carried a missense mutation[27].

In patients with (germline) FH mutations, papillary tumours are of type 2. Tumours of affected families have reduced or undetectable FH activity, and show an angiogenic phenotype[27]. These renal tumuors overexpress HIF proteins as well as products of the HIF-regulated target genes (like VEGF and GLUT1). Isaacs et al. reported that excess intracellular fumarate, due to loss of FH, upregulates VEGF and GLUT1 transcripts in a HIF-dependent manner[28]. They further demonstrated a link between fumarate dysregulation and impaired HIF hydroxylation, due to inhibited HIF prolyl hydroxylase (HPH) activity by fumarate. Because VHL recognition of HIF requires hydroxylation by $\mathrm{HPH}$, which is impaired in FH mutation-bearing tumours, HIF proteins accumulate and 
contribute to the development of these renal tumours. [28] Pollard et al. confirmed this pseudo-hypoxia phenomenon in a Fh1 "knock-out" mouse model. They showed induction of hypoxia pathway components, resulting from HIF protein overexpression, as a direct consequence of Fh1 inactivation[29].

It is clear that FH germline mutations are associated with the development of pRCC, but the role of $\mathrm{FH}$ alterations in sporadic kidney cancer remains to be determined since there is no conclusive evidence that somatic mutations of FH have a significant role in sporadic kidney cancers[26].

\subsection{Chromophobe renal cell carcinoma-Birt-Hogg-Dubé (BHD) tumour suppressor gene}

Birt-Hogg-Dubé (BHD) is a hereditary cancer syndrome characterized by kidney tumours in 15-25\% of the affected patients[30]. BHD syndrome was first characterized in 1977,[31] but renal manifestations were not described as part of BHD until Roth et al [32] suggested an association in 1993. In 1999, Toro et al [33] denominated BHD as a novel marker of kidney neoplasia. The predominant pathology of BHD-related kidney tumours is chromophobe[23].

The BHD gene, or FLCN, is located on the short arm of chromosome 17. In approximately $80 \%$ of BHD kindreds, a germline mutation is found. The gene contains a hotspot for germline mutations in a mononucleotide tract of eight cytosines at exon 11 and approximately all these mutations result in truncated BHD protein, folliculin $[23,30]$. Folliculin is suggested to have a tumour suppressor function, since germline mutations combined with somatic mutations or $\mathrm{LOH}$ resulted in truncation mutants of the folliculin protein. In addition, low to undetectable levels of BHD mRNA were found by Warren et al in kidney tumours from BHD patients, suggesting a classical TSG[34]. The identification of candidate genes involved in familial kidney cancers may provide insight into sporadic kidney cancers. Examination of the possible role of the BHD gene in sporadic chrRCC confirmed alteration of chromosome 17, but mutations in the BHD gene could not be detected[35,36]. Recently Gad et al. published the (first) report of four different somatic BHD mutations in two (out of 46 chrRCC) sporadic chrRCC. [37]. Interestingly, no exon 11 hotspot mutations as reported in BHD patients have been observed, indicating difference in tumourigenesis in the familial and sporadic form, as in pRCC.

A lack (or low incidence) of somatic mutations in the BHD gene suggests that the folliculin pathway may contain other causative genes in sporadic chrRCC, or other mechanisms of inactivation may be involved. 


\subsection{Renal cell carcinoma in tuberous sclerosis - Tuberous sclerosis complex 1 (TSC1) and 2 (TSC2) tumour suppressor genes}

Tuberous sclerosis complex (TSC) patients have an increased risk for development of malignant RCC.[38,39]. In contrast to the other genetic syndromes, renal carcinomas in TSC constitute a heterogeneous group, including $\mathrm{CCRCC}, \mathrm{pRCC}$, and chrRCC[40].

TSC is a genetic disorder, affecting 1 in 6,000-11,000 live births, and is characterized by hamartomatous lesions in multiple organs, involving the kidney in $\sim 60 \%$ of which RCC occurs in 4\%[41-43].

TSC has been linked to the germline inactivating mutations of either of two tumour suppressor genes TSC1 (9q34), coding for hamartin or TSC2 (16p13.3), coding for tuberin. TSC2 forms a complex with TSC1 which functions to integrate growth factor, nutrient, and energy depletion signals with the energy requiring protein translation apparatus through the regulation of the mTOR (mammalian target of rapamycin) protein.[38,39] The TSC1/TSC2 protein complex inhibits mTOR and is involved in signalling pathways that regulate cell growth[40].

Only small numbers of TSC patients with RCC have been studied because of the low prevalence. However, rodent models (the Eker rat / knockout mice) show evidence for a role of the TSC1 and TSC2 orthologues in RCC[44,45].

A report concerning TSC mutations in sporadic RCC did not support a role for TSC inactivation in sporadic tumourigenesis. They found LOH in 8 out of 33 cases, however expression (of the other allele) was confirmed by RT-PCR[46].

Interestingly, Liu et al. reported that loss of Tsc-2 tumour suppressor gene, in Eker rats which retain wild-type Vhl, up-regulates VEGF via a HIF2 $\alpha$-mediated mechanism. In contrast to human RCC, the Tsc-2 tumour suppressor gene is the primary target for RCC in rodents. They suggest that loss of Tsc- 2 and VHL tumour suppressor gene function have similar consequences in Eker rats and humans respectively. This marks the HIF-regulated pathway to be important for the development of RCC in different species and in tumours with different molecular etiologies[47].

\subsection{Clinical applications}

A growing understanding of the underlying molecular biology of RCC has established new potential therapies. The most significant progress has been made in the area of agents targeting the angiogenesis pathway (see chapter 3 ).

It has been suggested that the resistance of RCC to chemo- and radiotherapy might be partly due to increased levels of the transcription factor nuclear factor $\mathrm{KB}$ (NFKB) $[48,49]$. Loss of VHL leads, through HIF- $\alpha$ dependent and HIF- $\alpha$ independent pathways, to increased NFKB activity and resistance to apoptosis[50-53]. These findings support the idea that drugs targeting HIF or HIF-responsive gene products should be effective in the treatment of renal carcinomas. A number of drugs have been identified that indirectly down-regulate HIF- $\alpha$, including drugs that inhibit mTOR[54], HSP90[55] and histone deacetylases[56]. mTOR is a downstream component in the 
$\mathrm{P} 13 \mathrm{~K} /$ Akt pathway and has been identified as an upstream activator of HIF, enhancing translation of the HIF mRNA and preventing degradation[57,58]. Overexpressed wildtype histone deacetylase 1 downregulates expression of TP53 and VHL tumour suppressor genes and stimulates angiogenesis[56]. HSP90 stabilizes HIF-1 $\alpha$, EGFR and cMET[59].

Other VEGFR targeted tyrosine kinase inhibitors (Pazopanib, AG013736, VEGF-trap) and mTOR inhibitor (RAD001) are still under study in RCC[60-62]. Through combining agents that target different points in the VHL-hypoxia-inducible gene pathway the activity of the targeted approach to RCC therapy may be enhanced[63].

In addition, a number of approaches are under study to block the signal transduction pathway in c-Met associated tumours[64,65]. Small molecules inhibiting C-MET (PHA665752, SU11274) have been shown to inhibit proliferation and growth of $C-M E T$ overexpressing cell lines in mouse xenograft models[66,67].

Further gene expression profiling of tumour specimens will help to identify new candidate genes or patterns of gene expression that can predict response to new and preexisting therapies, leading to an appropriate patient selection for certain therapies.

Combinations of FDA-approved and other targeted therapies may overcome resistance that develops with single-agent therapy. Also combinations of targeted agents and cytokine therapy are under investigation. Phase I and II trials evaluating the safety and efficacy of these combinations are underway.

\section{Epigenetics of RCC}

Recently, it has become clear that gene function can be altered by epigenetic alterations. Epigenetics refers to mechanisms that initiate and maintain heritable (reversible) changes of gene expression and gene function in an inheritable manner without changing the sequence of the genome[68,69]. Epigenetic regulation of gene expression is organized at the level of DNA (postreplicative DNA methylation), RNA (RNA interference (RNAi)), and protein (posttranslational histone modifications and polycomb group (PcG) protein complexes, the latter mediate long-term transcriptional silencing)[6871].

DNA methylation is the most widely investigated epigenetic hallmark involving a postreplicative covalent DNA modification, whereby a methyl group is added to the cytosine ring by DNA methyltransferases (DNMTs) to form methyl-cytosine. In mammalian cells, DNA methylation occurs at so-called CpG dinucleotides, cytosines preceding a guanine[72]. CpG dinucleotides are not uniformly distributed throughout the human genome, the prevalence is low, except in CpG-rich regions called CpG islands, which span the $5^{\prime}$ end region (promoter, untranslated region, exon 1) of approximately half of all genes, and within repeat sequences and transposable elements[73]. Methylation of cytosines enables the formation of a complex of proteins, including methyl cytosine-binding proteins (MBDs), DNMTs, histone deacetylases (HDACs), and histone 
methyl transferases (HMTs) (see figure 1) to mediate the transcriptional repressive activities of DNA methylation $[69,72,74-76]$.

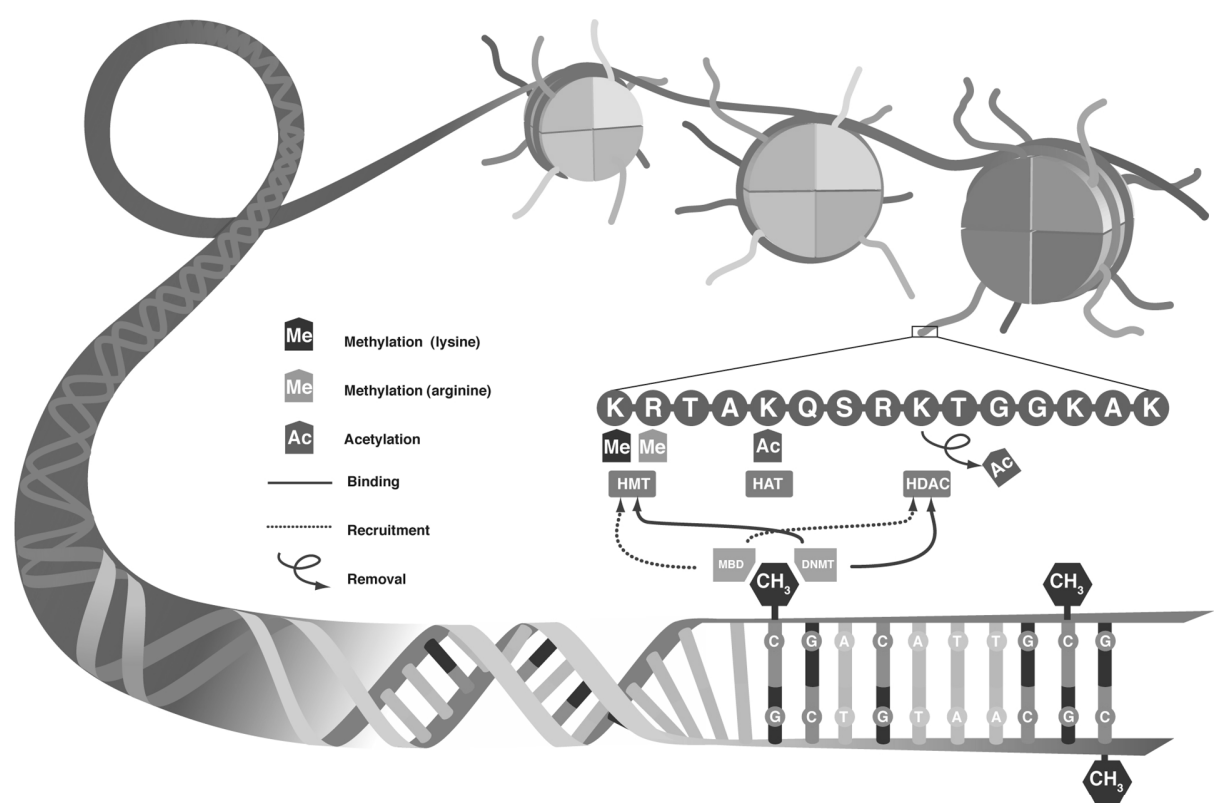

Figure. 1:. Epigenetic modifications and their interplay

The two main epigenetic hallmarks are postreplicative DNA methylation and posttranslational histone modifications, which both play a role in gene silencing and interact to repress gene transcription. (Additional histone modifications are known but not depicted here; histone methylation and acetylation are the most understood.)

DNA methylation is catalyzed by DNA methyltransferases (DNMTs) and characterized by the addition of a methyl group $(\mathrm{CH} 3)$ to a cytosine preceding a guanine $(\mathrm{CpG})$. Histone (de)acetylation results from an imbalance between histone deacetylases (HDACs) and histone acetyltransferases (HATs), whereby deacetylation is associated with gene repression. HDACs and histone methyltransferases (HMTs) are recruited by methylbinding proteins (MBPs), which bind methylated CpGs, or are directly bound to DNMTs to epigenetically modify histone tail residues and regulate gene expression. For full colour picture see page 167.

DNA methylation is linked to aberrant patterns of post-translational histone modifications $[75,77]$, including acetylation, methylation, phosphorylation, ubiquitination, and sumoylation $[68,69,75]$. These modifications occur primarily at specific positions within the $\mathrm{N}$-terminal histone tails[78]. Specific combinations of histone modifications confer to the overall expression status of a certain chromatin region, which is known as the 'histone code' hypothesis[75]. Modifications on histones are dynamic and rapidly changing, depending on the signaling conditions within the cell[78].

The vast majority of all modifications remain poorly understood, although considerable progress in the understanding of histone (de)acetylation and (de)methylation has been made recently. An imbalance between histone deacetylases (HDACs) and histone acetyltransferases (HATs) results in (de)acetylation of histone lysine residues, whereby 
deacetylation is associated with gene repression. In addition, histone lysine methylation (mono-, di-, and trimethyl lysine) and arginine methylation (mono-, and (a)symmetrical dimethyl arginine) are catalyzed by histone methyltransferases (HMTs). Unlike histone acetylation, histone methylation has long been considered as a permanent modification. However, recent discovery of more than ten (potential) histone demethylases, mediating the reversal of methylation at specific histone residues, has been documented $[79,80]$.

Both histone modifications contribute to both active and repressive effects on chromatin function, depending on which residue is modified[81]. HDACs and HMTs are both associated with complexes involving DNMTs and MBDs, mediating DNA packaging and gene expression[76]. Depending on the histone residue modified, and the interaction with DNA methylation to form a complex regulatory network, it correlates (among other effects) with chromatin structure/accessibility, transcriptional activity and genome function $[70,78,82]$.

The importance of epigenetics in the process of tumourigenesis has been increasingly discovered during the last decades and has been accepted as a true alternative for mutations and deletions associated with a loss of gene function that can provide a selective advantage for neoplastic cells. Epigenetic changes could well be as important as changes in DNA sequence or copy number in altering gene expression, and the interplay between genetics and epigenetics during the progression of cancer is becoming more and more a focus of research interest[83-85]. Regarding RCC, mutations do not cover $100 \%$ of the cases and are less often found in sporadic RCC compared to heritable RCC. Also, sporadic cancers are known to arise from multiple (epi)genetic events, and additional aberrations remain to be discovered. Therefore, promoter hypermethylation of genes is thought to be involved in sporadic and/ or hereditary forms of RCC. Here, we are focusing on the role of promoter methylation of tumour suppressor genes in the three most prevalent histological types of RCC, ccRCC, pRCC and chrRCC. Recently, many (candidate) tumour suppressor genes silenced by DNA methylation and associated with one or more histological subtypes have been reported for primary RCC cases (Table 1a) and/or analyzed in RCC cell lines (Table 1b).

Different techniques to analyze methylation were used to obtain the gene promoterspecific methylation data. An initial approach includes restriction enzyme digestion with Southern hybridization techniques, based on the (in)ability of methylation sensitive restriction enzymes to digest methylated cytosines. Later, bisulfite treatment of DNA, which converts unmethylated cytosines to uracil, whereas methylated cytosines remain unchanged, allowed combined bisulfite restriction analysis (COBRA), (nested) methylation specific PCR (MSP), and bisulfite sequencing[75]. MSP is a very sensitive approach to detect DNA methylation, enabling detection of one methylated allele in a background of 1,000 unmethylated alleles, while nested MSP allows detection of one methylated allele in 50,000 unmethylated alleles[86].

The genes reported to be associated with RCC (Tables $1 \mathrm{a}$ and $1 \mathrm{~b}$ ) can be grouped by the biological function/pathways involved in tumourigenesis and/or may be important 
targets for anti tumour therapy, i.e. interference in cell cycle, DNA-repair mechanisms, extra-cellular matrix molecules with adhesion and/or invasion mechanisms, angiogenesis, apoptosis, metabolism, proliferation, transport, WNT-signaling, or genes with a known role in the oncogenesis of other types of cancer, without a known specific function.

\subsection{Methylation of the VHL promoter}

Somatic VHL mutations are found in $50-75 \%$ of sporadic ccRCC $[9-11,87]$. The absence of VHL mutations in a proportion of sporadic ccRCCs indicates a role for alternative methods of VHL inactivation[88]. Herman et al. investigated VHL promoter $\mathrm{CpG}$ island methylation and associated inactivation by RT-PCR and restriction enzyme analysis and Southern hybridization techniques[74]. None of the examined VHL hypermethylated tumours expressed the VHL gene and treatment of a renal cell culture line with 5-aza2'-deoxycytidine (5-aza-dc, a demethylating agent) resulted in reexpression of the VHL gene. Methylation analysis of the wildtype VHL alleles revealed promoter methylation in $19 \%$ of the examined RCC tumours. These were the first data suggesting that aberrant DNA methylation of the VHL gene, in addition to $\mathrm{LOH}$ or gene mutations, is involved in ccRCC carcinogenesis. These data were confirmed by other groups reporting VHL methylation in 20\%[89], $16 \%[90]$, and $17 \%[91]$ in ccRCC.

In addition to CCRCC, involvement of VHL promoter hypermethylation in other subtypes is mostly unknown[90,91]. As Van Houwelingen et al. reported VHL mutations also in other subtypes of RCC[8], Banks et al. reported methylation of VHL in PRCC analyzed by MSP[89].

\subsection{Methylation of the FH promoter}

One report regarding epigenetic silencing of $F H$ in renal cancers has been published, indicating absence of $F H$ promoter $\mathrm{CpG}$ island methylation as analyzed by bisulfite sequencing, in fifteen pRCCs [90].

\subsection{Methylation of the BHD promoter}

So far, only three groups investigated BHD (FLCN) promoter methylation and associated silencing in chrRCC $[36,37,92]$. da Silva et al. analyzed promoter methylation in twenty primary RCCs by COBRA and bisulfite sequencing. Promoter hypermethylation was not detected in the primary RCCs[92] and six tested RCC cell lines. In addition, no significant changes in expression after treatment of the demethylating agent 5-azadc[92] were observed. In contrast, Khoo et al. observed methylation in a wide spectrum of primary renal tumours, using MSP: $11 \%$ of $\operatorname{chrRCC}(n=9), 36 \%$ in pRCC $(n=11)$, and $33 \%$ in ccRCC $(n=12)$, while none of the tested kidney cancer cell lines showed methylation [36]. On the other hand, Gad et al. analyzed methylation status of the BHD promoter using restriction enzyme analysis on 39 chrRCC and seven ccRCC, but no evidence of promoter methylation was found[37]. 
Table 1a: Overview of TSG methylation frequencies in RCC

\begin{tabular}{|c|c|c|c|c|c|c|c|c|}
\hline Function & Gene & Map locus & $\begin{array}{l}\text { RCC } \\
(\%)\end{array}$ & $\begin{array}{l}\text { ccRCC } \\
(\%)\end{array}$ & $\begin{array}{l}\text { pRCC } \\
(\%)\end{array}$ & $\begin{array}{l}\text { chrRCC } \\
(\%)\end{array}$ & $\begin{array}{l}\text { Norm } \\
(\%)\end{array}$ & Ref. \\
\hline \multirow[t]{6}{*}{ Adhesion } & $\mathrm{CDH1}$ & $16 q 22.1$ & - & $\begin{array}{l}55 \\
(14- \\
83)\end{array}$ & $\begin{array}{l}36 \\
(16-69)\end{array}$ & $\begin{array}{l}13^{*} \\
(0-20)\end{array}$ & $8 * *$ & {$[90,95,98]$} \\
\hline & $\mathrm{CDH} 13$ & $\begin{array}{l}16 q 24.2- \\
q 24.3\end{array}$ & 3 & - & - & - & - & [96] \\
\hline & CTNNB1 & $3 p 21$ & - & 0 & 0 & $0 *$ & $0 * *$ & [98] \\
\hline & $E P B 41 L 3$ & 8p11.3 & - & 45 & - & - & - & [97] \\
\hline & $J U P$ & $17 q$ & $\begin{array}{l}87 \\
(83- \\
91)\end{array}$ & - & - & - & $\begin{array}{l}10 \\
(9-11)\end{array}$ & [93] \\
\hline & LSAMP & $3 q 13.2-q 21$ & n.a & 26 & - & - & - & [94] \\
\hline Angio-genesis & $V H L$ & $3 p 26-25$ & 5 & $\begin{array}{l}18 \\
(16- \\
20)\end{array}$ & $\begin{array}{l}12 * \\
(0-50)\end{array}$ & $0 *$ & $0^{*}$ & $\begin{array}{l}{[74,89,90,91,} \\
106]\end{array}$ \\
\hline \multirow[t]{5}{*}{ Apoptosis } & APAF1 & $12 q 23$ & 97 & - & - & - & 15 & [99] \\
\hline & CASP8 & $2 q 33-q 34$ & $\begin{array}{l}6 \\
(0-16)\end{array}$ & - & - & - & 0 & {$[96,99]$} \\
\hline & $D A P K 1$ & $9 q 34.1$ & $\begin{array}{l}35 \\
(24- \\
41)\end{array}$ & - & - & - & 5 & {$[96,99]$} \\
\hline & $R A R B$ & $3 p 24$ & - & $\begin{array}{l}10 \\
(2-52)\end{array}$ & $\begin{array}{l}12 \\
(0-20)\end{array}$ & $\begin{array}{l}6^{*} \\
(0-17)\end{array}$ & $0 * / * *$ & {$[90,95,96,98]$} \\
\hline & $X A F 1$ & $17 p 13.2$ & $\begin{array}{l}14 \\
(10- \\
30)\end{array}$ & - & - & - & - & {$[100,101]$} \\
\hline \multirow[t]{5}{*}{ Cell cycle } & $\begin{array}{l}\text { CDKN2 } \\
\left(P 14^{A R F}\right)\end{array}$ & $9 p 21$ & 71 & $\begin{array}{l}12 \\
(10- \\
23)\end{array}$ & $\begin{array}{l}18^{*} \\
(8-33)\end{array}$ & $\begin{array}{l}27^{*} \\
(10- \\
100)\end{array}$ & $\begin{array}{l}23 * / * * \\
(0-27)\end{array}$ & {$[90,91,95,98]$} \\
\hline & $\begin{array}{l}\text { CDKN2 } \\
\left(P 16^{I N K 4}\right)\end{array}$ & $9 p 21$ & $\begin{array}{l}5 \\
(0-7)\end{array}$ & $\begin{array}{l}10 \\
(0-35)\end{array}$ & $\begin{array}{l}13 * \\
(0-33)\end{array}$ & $\begin{array}{l}9 * \\
(0-50)\end{array}$ & $0 * / * *$ & $\begin{array}{l}{[90,91,95,96,} \\
98,102,106]\end{array}$ \\
\hline & $\begin{array}{l}F L C N \\
(B H D)\end{array}$ & $17 p 11.2$ & - & $\begin{array}{l}20 \\
(0-33)\end{array}$ & 36 & $\begin{array}{l}2^{*} \\
(0-11)\end{array}$ & - & {$[36,37,92]$} \\
\hline & RASSF1 & $3 p 21.3$ & $\begin{array}{l}37 \\
(20- \\
98)\end{array}$ & $\begin{array}{l}51 \\
(23- \\
91)\end{array}$ & $\begin{array}{l}71^{*} \\
(44- \\
100)\end{array}$ & $\begin{array}{l}27^{*} \\
(0-40)\end{array}$ & $\begin{array}{l}78 * / * * \\
(0- \\
100)\end{array}$ & $\begin{array}{l}{[90,91,95,98,} \\
104- \\
108,110]]\end{array}$ \\
\hline & RASSF5 & $19 q 31.1$ & 19 & 32 & - & - & - & {$[94,96]$} \\
\hline DNA- repair & MGMT & $10 q 26$ & - & $\begin{array}{l}4 \\
(2-6)\end{array}$ & $\begin{array}{l}6 \\
(0-10)\end{array}$ & $0 *$ & $\begin{array}{l}10 * \\
(0-11)\end{array}$ & {$[90,95,96,98]$} \\
\hline \multirow[t]{2}{*}{ Invasion } & COL1A1 & $\begin{array}{l}17 q 21.31- \\
q 22\end{array}$ & - & 65 & $40^{*}$ & $50 *$ & - & [112] \\
\hline & TIMP3 & $\begin{array}{l}22 q 12.1- \\
q 13.2\end{array}$ & 78 & $\begin{array}{l}45 \\
(19- \\
71)\end{array}$ & $\begin{array}{l}38^{*} \\
(23-50)\end{array}$ & $\begin{array}{l}44^{*} \\
(0-100)\end{array}$ & $\begin{array}{l}21 * / * * \\
(0-24)\end{array}$ & $\begin{array}{l}{[90,91,95,98,} \\
111]\end{array}$ \\
\hline Krebs cycle & $S D H B$ & 1p36.1-p35 & 4 & - & - & - & - & [96] \\
\hline \multirow[t]{7}{*}{ Metabolism } & $A B C B 1$ & $7 q 21.1$ & - & 87 & 85 & 80 & $97 * *$ & [98] \\
\hline & ESR1 & $6 q 25.1$ & - & 67 & 77 & 60 & $77 * *$ & [98] \\
\hline & ESR2 & $14 q 23.2$ & - & 56 & 46 & 50 & $44^{* *}$ & [98] \\
\hline & FHIT & $3 p 14.2$ & - & 52 & 54 & 50 & $69 * *$ & [98] \\
\hline & GSTP1 & $11 q 13$ & - & $\begin{array}{l}8 \\
(6-12)\end{array}$ & $\begin{array}{l}18 \\
(15-20)\end{array}$ & $0 *$ & $0 * / * *$ & {$[90,95,98]$} \\
\hline & MTHFR & $1 p 36.3$ & - & 100 & 100 & 100 & $100 * *$ & [98] \\
\hline & PTGS2 & $1 q 25.2-q 25.3$ & - & 96 & 92 & 90 & $100 * *$ & [98] \\
\hline
\end{tabular}


Genetics and epigenetics of renal cell cancer

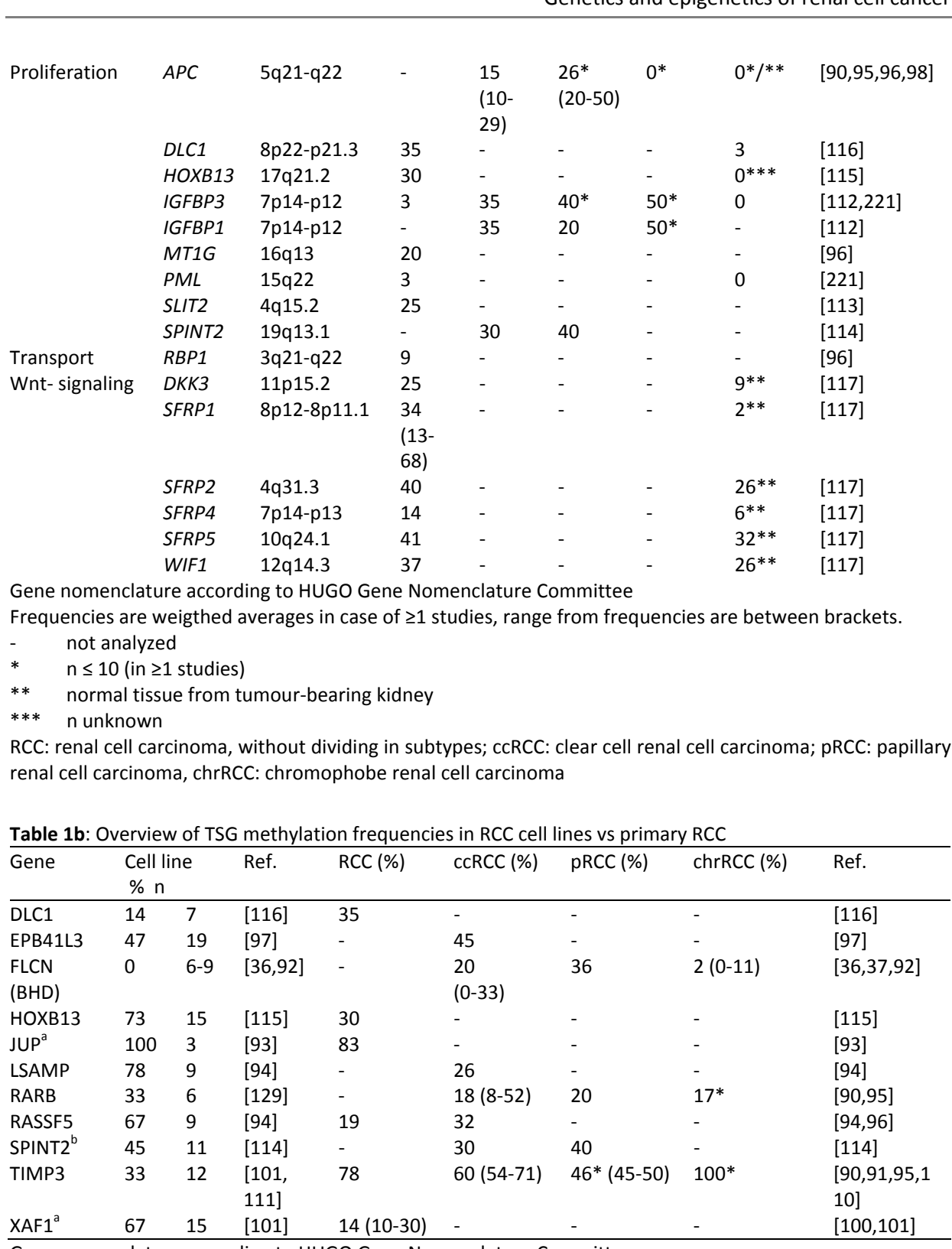

Gene nomenclature according to HUGO Gene Nomenclature Committee

Frequencies are weigthed averages in case of $\geq 1$ studies, range from frequencies are between brackets.

- not analyzed

* $\quad \mathrm{n} \leq 10$ (in $\geq 1$ studies)

a indirect measurement of methylation status; (mRNA) expression measurements

b indirect measurement of methylation status; upregulation after demethylation treatment

RCC: renal cell carcinoma, without dividing in subtypes; ccRCC: clear cell renal cell carcinoma; pRCC: papillary renal cell carcinoma, chrRCC: chromophobe renal cell carcinoma 


\subsection{Methylation of other tumour suppressor gene promoters}

In addition to the genes described above, other TSGs have been investigated (mainly by MSP), which are known to be involved in common cancer types, or which have been identified by microarray studies (see Tables $1 a$ and $1 b$ for details).

Several adhesion molecules (CDH1, CDH13, CTNNB1, EPB41L3, JUP, and LSAMP) were analyzed for promoter methylation in RCC, revealing methylation frequencies ranging from $3 \%$ (CDH13) to $87 \%$ (JUP) [90,93-98].

Many genes associated with apoptosis (APAF1, CASP8, DAPK1, RARB, and XAF1) have been recently investigated for their methylation frequencies in RCC, and approximately $10-30 \%$ methylation is observed for the majority of genes, except for APAF1, which is methylated in $97 \%$ of the tested RCCs $[90,95,96,98-101]$.

The cell cycle genes CDKN2a (p14ARF and p16INK4A) were methylated in $~ 10-20 \%$ of the RCC cases, while normal renal tissue was mostly unmethylated [90,91,95,96,98, 102]. The RAS association domain family 1A (RASSF1) gene at 3p21.3, though to be involved in the cell cycle, apoptosis and/or proliferation[103], has been implicated in the pathogenesis of a wide spectrum of tumours[103], including different RCC subtypes. Methylation is observed in $\sim 40-50 \%$ (weighted mean) in RCC[90,91,95,98,104108], however some studies detect high methylation frequencies in normal renal tissues. These histological normal samples were obtained from tumour-bearing kidneys, therefore a field effect[109] can cause this methylation and/or as reported before RASSF1 might be an early marker for RCC tumourigenesis[110]. In addition, RASSF1 methylation was detected in urine obtained from kidney cancer patients[95]. Potential importance of RASSF1 in early diagnosis is already highlighted in breast/lung cancer patients[103]. For a second RAS association domain family member, 5 (RASSF5), a methylation frequency of $\sim 20-30 \%$ has been ob-served[94,96].

The DNA-repair gene 06-methylguanine-DNA methyltransferase (MGMT) is methylated in $\sim 5 \%$ of the renal tumours $[90,95,96,98]$.

Genes involved in invasion/metastasis, such as COL1A1 and TIMP3, showed methylation in a high percentage ( $\sim 50 \%)$ in all RCC subtypes $[90,91,95,98,111,112]$.

For the Krebs cycle gene (SDHB) a very low methylation percentage (4\%) was observed[96].

The role of the detoxification gene Glutathione S-Transferase P1 (GSTP1) is not clear in RCC, but is it is methylated in $10-15 \%$ of the tumours $[90,95,98]$. Several other metabolism genes (ABCB1, ESR1, ESR2, FHIT, MTHFR, and PTGS2) have been analyzed by costa et al. by MSP, which are all methylated in high percentages ( $\geq 50 \%)$, however, also the normal renal samples show frequent methylation [98].

A methylation frequency of $\sim 30 \%$ is reported for several proliferation genes (see table 1a for details) $[90,95,96,98,99,112-116]$. Also a gene involved in transport of retinol (RBP1) was analyzed and a methylation frequency of $9 \%$ was observed[96].

Very recently, Urakami et al reported methylation analyses of six WNT- antagonist family genes (SFRP1, SFRP2, SFRP4, SFRP5, WIF1, and DKK3) in RCC (mostly ccRCC), 
analyzed by MSP. In this study all Wnt antagonist genes had significantly higher methylation frequencies in RCC compared to matched normal renal tissue. Methylation frequencies RCC versus normal were: SFRP1: $13 \%$ vs. $1,5 \%$, SFRP2: $40 \%$ vs. $25.5 \%$, SFRP4: $13.5 \%$ vs. $5.5 \%$, SFRP5: $41 \%$ vs. $31.5 \%$, WIF1: $37 \%$ vs. $25.5 \%$, and DKK $3: 25.5 \%$ vs. $9 \%$ (all p 0.005 ). mRNA expression for most WNT-antagonists was increased in two (out of two) RCC cell lines after treatment with 5-aza-2'deoxycytidine and confirmed to be methylated by bisulfite sequencing[117].

\subsection{Variables in promoter methylation studies}

Comparison of data from different studies on methylated tumour suppressor genes in RCC (Tables $1 a$ and $1 b$ ) is complicated by a considerable number of variables. First, instead of considering different subtypes of kidney cancer, RCC is considered as one single entity in some studies. Since RCCs are heterogeneous in histology, clinical behavior, genotype, prognosis and response to therapy, data should be presented for the different subtypes. An interpretation of the diagnostic or therapeutic value of these results is only possible when subtyping is taken into account. Recently, Costa et al. emphasized this importance of subtyping by reporting a gene panel which could discriminate between RCC subtypes by the presence/absence of methylation[98]. In addition to subtyping, consideration of tumour grade/stage is of importance in interpreting (epi)genetic alterations in RCC. The composition of tumour series used to study (epi)genetic alterations in RCC varies between different research groups, therefore it is important to include this variable.

In addition, different methods of measuring promoter methylation can account for part of the differences in results. Since MSP is very sensitive, especially when nested PCR has been performed [86], results might differ from those obtained by restriction enzyme-based methods. Furthermore, the position of measuring promoter methylation in a certain $\mathrm{CpG}$ island might differ between studies. Therefore, optimal and consensus primer location and design is critical to achieve reliable results[118].

Finally, when analyzing promoter methylation, the origin of normal renal tissue should be considered[109], and the number of RCC cases investigated should be sufficient to draw valid conclusions.

\subsection{Clinical applications}

DNA methylation markers have a promising future in clinical diagnostics, since DNA methylation changes have been reported to occur early in carcinogenesis[119] and early detection of disease results in an improved clinical outcome. In case of RCC, clinical signs and symptoms are often not useful in making an early diagnosis. The classic triad of pain, hematuria and a palpable flank mass is encountered in only $10 \%$ of patients and is usually associated with advanced disease[120]. Since identification of patients with organ-confined RCC is important for long-term disease-free survival after radical or partial nephrectomy[121], development of novel diagnostic noninvasive 
approaches for the early detection of kidney cancer is imperative, especially in patients with inherited predisposition. Cancer specific DNA methylation patterns can be found in detached tumour cells in bodily fluids, such as blood and urine and detection of methylated DNA by the very sensitive (nested) MSP is noninvasive and relatively cheap.

Table 2 shows the results of studies of DNA methylation markers in urine and serum from patients with RCC $[91,95,117]$.

Furthermore, Urakami et al reported a higher frequency of methylation in serum DNA from RCC patients with higher-grade/stage tumours compared to lower-grade/stage tumours, indicating putative progression markers[117]. Other studies have demonstrated independent prognostic values for DNA methylation markers in RCC (PTEN[122], JUP[93], EPB41L3[97], APAF1 and DAPK1[99]), being predictors of poor prognostic outcome. Inactivation of RASSF1 is often because of promoter methylation and this gene has the potential to be used as a marker for early detection and tumour surveillance/monitoring of (RCC) cancer patients (along with a panel of other TSGs to obtain a higher sensitivity).

Obviously, methylation markers with a high sensitivity and specificity in urine and/or serum would be a significant improvement, as this would offer a non-invasive method for (early) diagnosis, prognosis, or response on therapy[109].

The quest for DNA methylation markers that are predictive for therapy response is still in its infancy[123-125], and such markers in RCC have not been reported. A better understanding of the molecular pathways involved in renal carcinogenesis and insight into the functional significance of methylation changes, will allow development of new therapeutic approaches by use of demethylating agents. Resistance of several tumour types, including RCC, for certain anti-cancer agents such as chemotherapeutics, might be a result of epigenetic mechanisms that regulate genes involved in resistance, e.g. proapoptotic genes silenced by DNA methylation and/or histone acetylation. Demethylating agents or HDAC inhibitors might reverse these repressive effects[126]. Reu et al. demonstrated augmentation of immune response by DNA methyltransferase 1 inhibitors, through reactivation of apoptosis-associated Interferon response gene XAF1[127]. The overcoming of resistance to Interferon-induced apoptosis in RCC cells by demethylating agents may have clinical significance for cytokine therapy response. Shang et al. reported a synergistic growth suppression of RCC (in vitro), caused by the DNA methyltransferase inhibitor, 5-aza-2'-deoxycytidine, and a chemotherapeutic agent, Paclitaxel (PTX). They suggest that 5-aza-2'-deoxycytidine could increase the susceptibility of RCC to PTX and that combination chemotherapy with this inhibitor and PTX might be a novel strategy to improve the clinical response rate of RCC[128]. 
Genetics and epigenetics of renal cell cancer

Table 2: Studies of DNA methylation markers in urine and serum from patients with RCC

\begin{tabular}{|c|c|c|c|c|c|c|}
\hline Samples & $\begin{array}{l}\text { DNA } \\
\text { source }\end{array}$ & Markers & $\begin{array}{l}\text { Analytical } \\
\text { sensitivity (\%) }\end{array}$ & $\begin{array}{l}\text { Clinical } \\
\text { Sensitivity (\%) }\end{array}$ & Specificity (\%) & Ref. \\
\hline 23 ccRCC, & urine & $A P C$ & $4 / 5(80)$ & $10 / 26(38)$ & 96 & [95] \\
\hline $1 \mathrm{pRCC}$ & & $\mathrm{CDH1}$ & $6 / 10(60)$ & $10 / 26(38)$ & 95 & \\
\hline $1 \mathrm{chrRCC}$, & & GSTP1 & $1 / 2(50)$ & $4 / 26(15)$ & 100 & \\
\hline 1 collecting & & MGMT & $0 / 1(0)$ & $2 / 26(8)$ & 100 & \\
\hline \multirow[t]{11}{*}{ duct } & & P16 & $4 / 6(67)$ & $9 / 26(35)$ & 100 & \\
\hline & & $R A R B$ & $4 / 9(44)$ & $8 / 26(31)$ & 91 & \\
\hline & & RASSF1 & 11/15 (73) & $17 / 26(65)$ & 89 & \\
\hline & serum & $A P C$ & $1 / 5(20)$ & $1 / 18(6)$ & 97 & \\
\hline & & $\mathrm{CDH1}$ & $06 / 10(60)$ & 6/18 (33) & 93 & \\
\hline & & GSTP1 & $0 / 2(0)$ & $1 / 18(6)$ & 100 & \\
\hline & & MGMT & $0 / 1(1)$ & $0 / 18(0)$ & 97 & \\
\hline & & P14 & $1 / 4(25)$ & $1 / 18(6)$ & 97 & \\
\hline & & P16 & $3 / 6(50)$ & $4 / 18(22)$ & 100 & \\
\hline & & $R A R B$ & $1 / 9(11)$ & $1 / 18(6)$ & 100 & \\
\hline & & RASSF1 & 2/15 (13) & $2 / 18(11)$ & 97 & \\
\hline 35 ccRCC, & urine & $A P C$ & $7 / 7$ (100) & $9 / 50(18)$ & 100 & [91] \\
\hline $6 \mathrm{pRCC}$ & & P14 & $8 / 8(100)$ & $9 / 50(18)$ & 100 & \\
\hline \multirow[t]{4}{*}{$2 \mathrm{chrRCC}$} & & P16 & $4 / 5(80)$ & $5 / 50(10)$ & 100 & \\
\hline & & RASSF1 & $23 / 24(95)$ & $26 / 50(52)$ & 100 & \\
\hline & & TIMP3 & $23 / 26(88)$ & $30 / 50(60)$ & 100 & \\
\hline & & $V H L$ & $6 / 6(101)$ & $6 / 50(12)$ & 100 & \\
\hline \multirow[t]{6}{*}{$33 \mathrm{RCC}$} & serum & DKK3 & $6 / 10(60)$ & $9 / 33(27)$ & 100 & [117] \\
\hline & & SFRP1 & $5 / 8(62)$ & $9 / 33(27)$ & 100 & \\
\hline & & SFRP2 & $12 / 13(92)$ & $7 / 33(21)$ & 100 & \\
\hline & & SFRP4 & $5 / 15(33)$ & $8 / 33(24)$ & 100 & \\
\hline & & SFRP5 & $9 / 16(56)$ & $15 / 33(45)$ & 100 & \\
\hline & & WIF1 & $5 / 13(38)$ & $9 / 33(27)$ & 100 & \\
\hline
\end{tabular}

Gene nomenclature according to HUGO Gene Nomenclature Committee

Analytical sensitivity is defined as the fraction of cases in which methylation of a marker is found in serum or urine for cases with confirmed methylation of the same marker in the associated tumour.

Clinical sensitivity is defined as the fraction of confirmed cases of disease, in which methylation of a marker is found in serum or urine, regardless of whether methylation of that marker is present in the associated tumour.

Specificity is defined as the fraction of controls without the disease that show a lack of detectable methylation in serum or urine.

RCC: renal cell carcinoma, without dividing in subtypes; ccRCC: clear cell renal cell carcinoma; pRCC: papillary renal cell carcinoma, chrRCC: chromophobe renal cell carcinoma

\section{Conclusion}

RCC is the most important tumour of the kidney in adults. In etiological, biological and clinical characteristics it represents a heterogeneous entity, which, through lack of clinical symptoms, may be difficult to detect in an early stage. Surgery is the first treatment of choice, and most successful if the tumour is confined to the kidney. Systemic treatment of RCC has made considerable progress in patients with metastatic 
RCC, e.g. by development of new emerging strategies (targeted agents) in recent years. Further studies are needed to address the questions regarding the efficacy of new targets and the efficacy of combination treatments.

Developing knowledge of genetic and epigenetic changes implicated in tumour development and behaviour is becoming increasingly important for advancing the efficacy of disease management.

Defective VHL functioning appears to be the key event, in both hereditary and sporadic cases. However, the variable nature of the resulting neoplasm is most likely strongly determined by the complex interplay of additional downstream modifications, among which the role of epigenetic alteration of gene expression is becoming more and more acknowledged.

Elucidation of epigenetic modifications in RCC holds great promise for early detection, prediction of sensitivity for non-surgical therapy, but also for therapeutical interventions through reversal of epigenetic gene silencing. However, for definitive progress to be made in this field of research, uniformity in study design and consensus in clinicopathological definitions and scientific methods is a prerequisite. 


\section{References}

1 Linehan, W.M. et al. (2004) Genetic basis of cancer of the kidney: disease-specific approaches to therapy. Clin Cancer Res 10, 6282S-6289S

2 Kim, W.Y. and Kaelin, W.G. (2004) Role of VHL gene mutation in human cancer. J Clin Oncol 22, 49915004

3 Seizinger, B.R. et al. (1988) Von Hippel-Lindau disease maps to the region of chromosome 3 associated with renal cell carcinoma. Nature 332, 268-269

4 Latif, F. et al. (1993) Identification of the von Hippel-Lindau disease tumour suppressor gene. Science 260, 1317-1320

5 Stolle, C. et al. (1998) Improved detection of germline mutations in the von Hippel-Lindau disease tumour suppressor gene. Hum Mutat 12, 417-423

6 Choyke, P.L. et al. (2003) Hereditary renal cancers. Radiology 226, 33-46

7 Mandriota, S.J. et al. (2002) HIF activation identifies early lesions in VHL kidneys: evidence for sitespecific tumour suppressor function in the nephron. Cancer Cell 1, 459-468

8 van Houwelingen, K.P. et al. (2005) Prevalence of von Hippel-Lindau gene mutations in sporadic renal cell carcinoma: results from The Netherlands cohort study. BMC Cancer 5, 57

9 Weiss, R.H. and Lin, P.Y. (2006) Kidney cancer: identification of novel targets for therapy. Kidney Int 69, 224-232

10 Zbar, B. et al. (2003) Studying cancer families to identify kidney cancer genes. Annu Rev Med 54, 217233

11 Gnarra, J.R. et al. (1994) Mutations of the VHL tumour suppressor gene in renal carcinoma. Nat Genet 7, 85-90

12 Kondo, K. et al. (2002) Comprehensive mutational analysis of the VHL gene in sporadic renal cell carcinoma: relationship to clinicopathological parameters. Genes Chromosomes Cancer 34, 58-68

13 Cohen, H.T. and McGovern, F.J. (2005) Renal-cell carcinoma. N Engl J Med 353, 2477-2490

14 Blankenship, C. et al. (1999) Alternate choice of initiation codon produces a biologically active product of the von Hippel Lindau gene with tumour suppressor activity. Oncogene 18, 1529-1535

15 Iliopoulos, O. et al. (1998) pVHL19 is a biologically active product of the von Hippel-Lindau gene arising from internal translation initiation. Proc Natl Acad Sci U S A 95, 11661-11666

16 Schoenfeld, A. et al. (1998) A second major native von Hippel-Lindau gene product, initiated from an internal translation start site, functions as a tumour suppressor. Proc Natl Acad Sci U S A 95, 8817-8822

17 Kondo, K. and Kaelin, W.G., Jr. (2001) The von Hippel-Lindau tumour suppressor gene. Exp Cell Res 264, 117-125

18 Gordan, J.D. and Simon, M.C. (2007) Hypoxia-inducible factors: central regulators of the tumour phenotype. Curr Opin Genet Dev 17, 71-77

19 Kaelin, W.G., Jr. (2007) The von Hippel-Lindau tumour suppressor protein and clear cell renal carcinoma. Clin Cancer Res 13, 680s-684s

20 Maxwell, P.H. et al. (1999) The tumour suppressor protein VHL targets hypoxia-inducible factors for oxygen-dependent proteolysis. Nature 399, 271-275

21 Clifford, S.C. et al. (2001) Contrasting effects on HIF-1alpha regulation by disease-causing pVHL mutations correlate with patterns of tumourigenesis in von Hippel-Lindau disease. Hum Mol Genet 10, 10291038

22 Zbar, B. et al. (1994) Hereditary papillary renal cell carcinoma. J Urol 151, 561-566

23 Sudarshan, S. and Linehan, W.M. (2006) Genetic basis of cancer of the kidney. Semin Oncol 33, 544-551 
24 Schmidt, L. et al. (1997) Germline and somatic mutations in the tyrosine kinase domain of the MET proto-oncogene in papillary renal carcinomas. Nat Genet $16,68-73$

25 Schmidt, L. et al. (1999) Novel mutations of the MET proto-oncogene in papillary renal carcinomas. Oncogene 18, 2343-2350

26 Sudarshan, S. et al. (2007) Mechanisms of disease: hereditary leiomyomatosis and renal cell cancer--a distinct form of hereditary kidney cancer. Nat Clin Pract Urol 4, 104-110

27 Tomlinson, I.P. et al. (2002) Germline mutations in FH predispose to dominantly inherited uterine fibroids, skin leiomyomata and papillary renal cell cancer. Nat Genet 30, 406-410

28 Isaacs, J.S. et al. (2005) HIF overexpression correlates with biallelic loss of fumarate hydratase in renal cancer: novel role of fumarate in regulation of HIF stability. Cancer Cell 8, 143-153

29 Pollard, P.J. et al. (2007) Targeted inactivation of fh1 causes proliferative renal cyst development and activation of the hypoxia pathway. Cancer Cell 11, 311-319

30 Linehan, W.M. and Zbar, B. (2004) Focus on kidney cancer. Cancer Cell 6, 223-228

31 Birt, A.R. et al. (1977) Hereditary multiple fibrofolliculomas with trichodiscomas and acrochordons. Arch Dermatol 113, 1674-1677

32 Roth, J.S. et al. (1993) Bilateral renal cell carcinoma in the Birt-Hogg-Dube syndrome. J Am Acad Dermatol 29, 1055-1056

33 Toro, J.R. et al. (1999) Birt-Hogg-Dube syndrome: a novel marker of kidney neoplasia. Arch Dermatol $135,1195-1202$

34 Warren, M.B. et al. (2004) Expression of Birt-Hogg-Dube gene mRNA in normal and neoplastic human tissues. Mod Pathol 17, 998-1011

35 Nagy, A. et al. (2004) Lack of mutation of the folliculin gene in sporadic chromophobe renal cell carcinoma and renal oncocytoma. Int J Cancer 109, 472-475

36 Khoo, S.K. et al. (2003) Inactivation of BHD in sporadic renal tumours. Cancer Res 63, 4583-4587

37 Gad, S. et al. (2006) Mutations in BHD and TP53 genes, but not in HNF1beta gene, in a large series of sporadic chromophobe renal cell carcinoma. Br J Cancer

38 Dorai, T. et al. (2006) Role of carbonic anhydrases in the progression of renal cell carcinoma subtypes: proposal of a unified hypothesis. Cancer Invest 24, 754-779

39 Mak, B.C. and Yeung, R.S. (2004) The tuberous sclerosis complex genes in tumour development. Cancer Invest 22, 588-603

40 Henske, E.P. (2005) Tuberous sclerosis and the kidney: from mesenchyme to epithelium, and beyond. Pediatr Nephrol 20, 854-857

41 Rakowski, S.K. et al. (2006) Renal manifestations of tuberous sclerosis complex: Incidence, prognosis, and predictive factors. Kidney Int 70, 1777-1782

42 O'Callaghan, F.J. et al. (2004) An epidemiological study of renal pathology in tuberous sclerosis complex. BJU Int 94, 853-857

43 O'Callaghan, F.J. et al. (1998) Prevalence of tuberous sclerosis estimated by capture-recapture analysis. Lancet 351, 1490

44 Kobayashi, T. et al. (1995) A germline insertion in the tuberous sclerosis (Tsc2) gene gives rise to the Eker rat model of dominantly inherited cancer. Nat Genet 9, 70-74

45 Kobayashi, T. et al. (1999) Renal carcinogenesis, hepatic hemangiomatosis, and embryonic lethality caused by a germ-line Tsc2 mutation in mice. Cancer Res 59, 1206-1211

46 Parry, L. et al. (2001) Analysis of the TSC1 and TSC2 genes in sporadic renal cell carcinomas. Br J Cancer $85,1226-1230$

47 Liu, M.Y. et al. (2003) Up-regulation of hypoxia-inducible factor 2alpha in renal cell carcinoma associated with loss of Tsc-2 tumour suppressor gene. Cancer Res 63, 2675-2680 
48 Oya, M. et al. (2003) Increased nuclear factor-kappa B activation is related to the tumour development of renal cell carcinoma. Carcinogenesis 24, 377-384

49 Oya, M. et al. (2001) Constitutive activation of nuclear factor-kappaB prevents TRAIL-induced apoptosis in renal cancer cells. Oncogene 20, 3888-3896

50 An, J. et al. (2005) VHL expression in renal cell carcinoma sensitizes to bortezomib (PS-341) through an NF-kappaB-dependent mechanism. Oncogene 24, 1563-1570

$51 \mathrm{Qi}, \mathrm{H}$. and Ohh, M. (2003) The von Hippel-Lindau tumour suppressor protein sensitizes renal cell carcinoma cells to tumour necrosis factor-induced cytotoxicity by suppressing the nuclear factor-kappaBdependent antiapoptotic pathway. Cancer Res 63, 7076-7080

52 An, J. and Rettig, M.B. (2005) Mechanism of von Hippel-Lindau protein-mediated suppression of nuclear factor kappa B activity. Mol Cell Biol 25, 7546-7556

53 Makino, Y. et al. (2001) Inhibitory PAS domain protein is a negative regulator of hypoxia-inducible gene expression. Nature 414, 550-554

54 Thomas, G.V. et al. (2006) Hypoxia-inducible factor determines sensitivity to inhibitors of mTOR in kidney cancer. Nat Med 12, 122-127

55 Neckers, L. (2002) Hsp90 inhibitors as novel cancer chemotherapeutic agents. Trends Mol Med 8, S5561

56 Kim, M.S. et al. (2001) Histone deacetylases induce angiogenesis by negative regulation of tumour suppressor genes. Nat Med 7, 437-443

57 Hudson, C.C. et al. (2002) Regulation of hypoxia-inducible factor 1alpha expression and function by the mammalian target of rapamycin. Mol Cell Biol 22, 7004-7014

58 Semenza, G.L. (2003) Targeting HIF-1 for cancer therapy. Nat Rev Cancer 3, 721-732

59 Isaacs, J.S. et al. (2002) Hsp90 regulates a von Hippel Lindau-independent hypoxia-inducible factor-1 alpha-degradative pathway. J Biol Chem 277, 29936-29944

60 Rini B, R.O., Bukowski R. (2005) AG-013736, a multi-target tyrosine kinase receptor inhibitor, demonstrates anti-tumour activity in a Phase 2 study of cytokine-refractory, metastatic renal cancer (RCC). $J$ clin oncol 23

61 Amato RJ, M.A., Khan M. (2006) A Phase II trial of RAD001 in patients with metastaic renal cell carcinoma (MRCC). J clin oncol 24

62 Dupont J, C.D., Gordon M. (2003) Phase I study of VEGF Trap in patients with solid tumours and lymphoma. Proc Am Soc Clin Oncol 22, 194

63 Kaelin, W.G., Jr. (2004) The von Hippel-Lindau tumour suppressor gene and kidney cancer. Clin Cancer Res 10, 6290S-6295S

64 Kong-Beltran, M. et al. (2004) The Sema domain of Met is necessary for receptor dimerization and activation. Cancer Cell 6, 75-84

65 Zhang, Y.W. et al. (2004) Met decoys: will cancer take the bait? Cancer Cell 6, 5-6

66 Christensen, J.G. et al. (2003) A selective small molecule inhibitor of c-Met kinase inhibits c-Metdependent phenotypes in vitro and exhibits cytoreductive antitumour activity in vivo. Cancer Res 63, 7345-7355

67 Sattler, M. et al. (2003) A novel small molecule met inhibitor induces apoptosis in cells transformed by the oncogenic TPR-MET tyrosine kinase. Cancer Res 63, 5462-5469

68 Callinan, P.A. and Feinberg, A.P. (2006) The emerging science of epigenomics. Hum Mol Genet 15 Spec No 1 , R95-101

69 Esteller, M. (2006) The necessity of a human epigenome project. Carcinogenesis 27, 1121-1125

70 Bernstein, B.E. et al. (2007) The mammalian epigenome. Cell 128, 669-681

71 Brock, M.V. et al. (2007) Cancer as a manifestation of aberrant chromatin structure. Cancer J 13, 3-8 
72 Herman, J.G. and Baylin, S.B. (2003) Gene silencing in cancer in association with promoter hypermethylation. N Engl J Med 349, 2042-2054

73 Wilson, A.S. et al. (2007) DNA hypomethylation and human diseases. Biochim Biophys Acta 1775, 138162

74 Herman, J.G. et al. (1994) Silencing of the VHL tumour-suppressor gene by DNA methylation in renal carcinoma. Proc Natl Acad Sci U S A 91, 9700-9704

75 Esteller, M. (2007) Cancer epigenomics: DNA methylomes and histone-modification maps. Nat Rev Genet 8, 286-298

76 Ting, A.H. et al. (2006) The cancer epigenome--components and functional correlates. Genes Dev 20, 3215-3231

77 Fahrner, J.A. et al. (2002) Dependence of histone modifications and gene expression on DNA hypermethylation in cancer. Cancer Res 62, 7213-7218

78 Kouzarides, T. (2007) Chromatin modifications and their function. Cell 128, 693-705

79 Lan, F. et al. (2007) A histone H3 lysine 27 demethylase regulates animal posterior development. Nature

80 Tan, H. et al. (2007) The JMJD2 members of histone demethylase revisited. Mol Biol Rep

81 Klose, R.J. and Zhang, Y. (2007) Regulation of histone methylation by demethylimination and demethylation. Nat Rev Mol Cell Biol 8, 307-318

82 Li, B. et al. (2007) The role of chromatin during transcription. Cell 128, 707-719

83 Balmain, A. et al. (2003) The genetics and genomics of cancer. Nat Genet 33 Suppl, 238-244

84 Jones, P.A. and Baylin, S.B. (2002) The fundamental role of epigenetic events in cancer. Nat Rev Genet 3, 415-428

85 Schuebel, K.E. et al. (2007) Comparing the DNA Hypermethylome with Gene Mutations in Human Colorectal Cancer. PLoS Genet 3, e157

86 Derks, S. et al. (2004) Methylation-specific PCR unraveled. Cell Oncol 26, 291-299

87 Foster, K. et al. (1994) Somatic mutations of the von Hippel-Lindau disease tumour suppressor gene in non-familial clear cell renal carcinoma. Hum Mol Genet 3, 2169-2173

88 Clifford, S.C. et al. (1998) Inactivation of the von Hippel-Lindau (VHL) tumour suppressor gene and allelic losses at chromosome arm $3 p$ in primary renal cell carcinoma: evidence for a VHL-independent pathway in clear cell renal tumourigenesis. Genes Chromosomes Cancer 22, 200-209

89 Banks, R.E. et al. (2006) Genetic and epigenetic analysis of von Hippel-Lindau (VHL) gene alterations and relationship with clinical variables in sporadic renal cancer. Cancer Res 66, 2000-2011

90 Dulaimi, E. et al. (2004) Promoter hypermethylation profile of kidney cancer. Clin Cancer Res 10, 3972 3979

91 Battagli, C. et al. (2003) Promoter hypermethylation of tumour suppressor genes in urine from kidney cancer patients. Cancer Res 63, 8695-8699

92 da Silva, N.F. et al. (2003) Analysis of the Birt-Hogg-Dube (BHD) tumour suppressor gene in sporadic renal cell carcinoma and colorectal cancer. J Med Genet 40, 820-824

93 Breault, J.E. et al. (2005) Methylation of the gamma-catenin gene is associated with poor prognosis of renal cell carcinoma. Clin Cancer Res 11, 557-564

94 Chen, J. et al. (2003) The t(1;3) breakpoint-spanning genes LSAMP and NORE1 are involved in clear cell renal cell carcinomas. Cancer Cell 4, 405-413

95 Hoque, M.O. et al. (2004) Quantitative detection of promoter hypermethylation of multiple genes in the tumour, urine, and serum DNA of patients with renal cancer. Cancer Res 64, 5511-5517

96 Morris, M.R. et al. (2003) Multigene methylation analysis of Wilms' tumour and adult renal cell carcinoma. Oncogene 22, 6794-6801 
97 Yamada, D. et al. (2006) Promoter hypermethylation of the potential tumour suppressor DAL-1/4.1B gene in renal clear cell carcinoma. Int J Cancer 118, 916-923

98 Costa, V.L. et al. (2007) Quantitative promoter methylation analysis of multiple cancer-related genes in renal cell tumours. BMC Cancer 7, 133

99 Christoph, F. et al. (2006) Promoter hypermethylation profile of kidney cancer with new proapoptotic p53 target genes and clinical implications. Clin Cancer Res 12, 5040-5046

100 Kempkensteffen, C. et al. (2007) Gene expression and promoter methylation of the XIAP-associated Factor 1 in renal cell carcinomas: Correlations with pathology and outcome. Cancer Lett

101 Lee, M.G. et al. (2006) Promoter CpG hypermethylation and downregulation of XAF1 expression in human urogenital malignancies: implication for attenuated p53 response to apoptotic stresses. Oncogene 25, 5807-5822

102 Sanz-Casla, M.T. et al. (2003) Loss of heterozygosity and methylation of p16 in renal cell carcinoma. Urol Res 31, 159-162

103 Agathanggelou, A. et al. (2005) Role of the Ras-association domain family 1 tumour suppressor gene in human cancers. Cancer Res 65, 3497-3508

104 Dreijerink, K. et al. (2001) The candidate tumour suppressor gene, RASSF1A, from human chromosome 3p21.3 is involved in kidney tumourigenesis. Proc Natl Acad Sci U S A 98, 7504-7509

105 Gonzalgo, M.L. et al. (2004) Molecular profiling and classification of sporadic renal cell carcinoma by quantitative methylation analysis. Clin Cancer Res 10, 7276-7283

106 Hori, Y. et al. (2007) Oxidative stress and DNA hypermethylation status in renal cell carcinoma arising in patients on dialysis. J Pathol 212, 218-226

107 Morrissey, C. et al. (2001) Epigenetic inactivation of the RASSF1A 3p21.3 tumour suppressor gene in both clear cell and papillary renal cell carcinoma. Cancer Res 61, 7277-7281

108 Yoon, J.H. et al. (2001) Hypermethylation of the CpG island of the RASSF1A gene in ovarian and renal cell carcinomas. Int I Cancer 94, 212-217

109 Cairns, P. (2007) Gene methylation and early detection of genitourinary cancer: the road ahead. Nat Rev Cancer 7, 531-543

110 Peters, I. et al. (2007) RASSF1A promoter methylation and expression analysis in normal and neoplastic kidney indicates a role in early tumourigenesis. Mol Cancer 6, 49

111 Bachman, K.E. et al. (1999) Methylation-associated silencing of the tissue inhibitor of metalloproteinase-3 gene suggest a suppressor role in kidney, brain, and other human cancers. Cancer Res 59, 798-802

112 Ibanez de Caceres, I. et al. (2006) Identification of novel target genes by an epigenetic reactivation screen of renal cancer. Cancer Res 66, 5021-5028

113 Astuti, D. et al. (2004) SLIT2 promoter methylation analysis in neuroblastoma, Wilms' tumour and renal cell carcinoma. Br J Cancer 90, 515-521

114 Morris, M.R. et al. (2005) Tumour suppressor activity and epigenetic inactivation of hepatocyte growth factor activator inhibitor type 2/SPINT2 in papillary and clear cell renal cell carcinoma. Cancer Res 65, 4598-4606

115 Okuda, H. et al. (2006) Epigenetic inactivation of the candidate tumour suppressor gene HOXB13 in human renal cell carcinoma. Oncogene 25, 1733-1742

116 Zhang, Q. et al. (2006) Aberrant methylation of the 8p22 tumour suppressor gene DLC1 in renal cell carcinoma. Cancer Lett

117 Urakami, S. et al. (2006) Wnt antagonist family genes as biomarkers for diagnosis, staging, and prognosis of renal cell carcinoma using tumour and serum DNA. Clin Cancer Res 12, 6989-6997

118 Brandes, J.C. et al. (2007) Optimal primer design using the novel primer design program: MSPprimer provides accurate methylation analysis of the ATM promoter. Oncogene 
119 Laird, P.W. (1997) Oncogenic mechanisms mediated by DNA methylation. Mol Med Today 3, 223-229

120 Baylin, S.B. et al. (1998) Alterations in DNA methylation: a fundamental aspect of neoplasia. Adv Cancer Res 72, 141-196

121 Pantuck, A.J. et al. (2000) Incidental renal tumours. Urology 56, 190-196

$122 \mathrm{Kim}, \mathrm{H} . \mathrm{L}$. et al. (2005) Using tumour markers to predict the survival of patients with metastatic renal cell carcinoma. J Urol 173, 1496-1501

123 Wei, S.H. et al. (2002) Methylation microarray analysis of late-stage ovarian carcinomas distinguishes progression-free survival in patients and identifies candidate epigenetic markers. Clin Cancer Res 8, 2246-2252

124 Esteller, M. et al. (2000) Inactivation of the DNA-repair gene MGMT and the clinical response of gliomas to alkylating agents. N Engl J Med 343, 1350-1354

125 Esteller, M. et al. (2002) Hypermethylation of the DNA repair gene O(6)-methylguanine DNA methyltransferase and survival of patients with diffuse large B-cell lymphoma. J Nat/ Cancer Inst 94, 26-32

126 Bar-Sela, G. et al. (2007) Histone deacetylase inhibitor and demethylating agent chromatin compaction and the radiation response by cancer cells. Cancer J 13, 65-69

127 Reu, F.J. et al. (2006) Overcoming resistance to interferon-induced apoptosis of renal carcinoma and melanoma cells by DNA demethylation. J Clin Oncol 24, 3771-3779

128 Shang, D. et al. (2007) Demethylating agent 5-aza-2'-deoxycytidine enhances susceptibility of renal cell carcinoma to paclitaxel. Urology 69, 1007-1012

129 X.F.Wang. et al. (2005) Epigenetic modulation of retinoi acid receptor beta2 by the histone deacetylase inhibitor MS-275 in human renal cell carcinoma. Clin Cancer Res 11, 3535-42 


\section{Chapter 8}

\section{Prognostic significance of Gremlin1 (GREM1) promoter CpG island hypermethylation in clear cell renal cell carcinoma}

Iris J.H. van Vlodrop, Marcella M.L. Baldewijns, Kim M. Smits, Leo J. Schouten, Leander van Neste, Wim van Criekinge, Hein van Poppel, Evelyne Lerut, Kornel Schuebel, Nita Ahuja, James G. Herman, Adriaan P. de Bruïne, and Manon van Engeland Submitted for publication 


\begin{abstract}
Gremlin1 (GREM1), a bone morphogenetic protein (BMP) antagonist and putative angiogenesis-modulating tumour suppressor gene, is silenced by promoter $\mathrm{CpG}$ island hypermethylation in several human malignancies. GREM1 promoter CpG island methylation in clear cell renal cell carcinoma (ccRCC)and its impact on tumour characteristics and clinical outcome has not yet been evaluated.

We analyzed three loci (GREM1 region-i, -ii, -iii) within the GREM1 promoter CpG island for CPG methylation by methylation specific PCR (MSP) and/or bisulfite sequencing in four ccRCC cell lines, and in tumour samples derived from two independent ( $n=150$ and $n=185$ ), well characterized ccRCC patients series. Results were correlated with clinicopathological- and angiogenesis parameters. All statistical tests were twosided.

Bisulfite sequencing and MSP analysis of ccRCC cell lines showed dense methylation of the GREM1 promoter CpG island, which was associated with absence of GREM1 mRNA. Tumour specific GREM1 promoter CpG island methylation prevalence in ccRCC varied between the three loci: $55 \%, 24 \%$, and $20 \%$ methylation for regions $\mathrm{i}$, ii, and iii, respectively. GREM1-region iii methylation was associated with increased tumour size $(p=0.02)$, stage $(p=0.013)$, Fuhrman grade $(p=0.04)$, tumour cell proliferation $(p=0.001)$, endothelial cell proliferation $(p=0.0001)$, and decreased mean vessel density $(p=0.001)$. In the univariate analysis, GREM1-region iii methylated cCRCCs had a significant worse survival as compared to unmethylated ccRCCs (hazard ratio (HR) $=2.35$ (95\% Cl: 1.294.28)), but not in the multivariate analysis ( $\mathrm{HR}=0.88,95 \% \mathrm{Cl}: 0.45-1.74)$. In a validation series, GREM1-region iii methylation was associated with increased Fuhrman grade $(p=0.03)$ and decreased overall survival $(p=0.001)$ in both univariate and multivariate analyses ( $\mathrm{HR}=2.32,95 \% \mathrm{Cl}: 1.52-3.53$ and $\mathrm{HR}=2.27,95 \% \mathrm{Cl}$ : 1.44-3.59, respectively).

The strong correlation between GREM1-region iii promoter methylation and increased malignancy in two large independent series of ccRCCs as well as a correlation with active angiogenesis indicates a putative role for GREM1 in CCRCC carcinogenesis and tumour angiogenesis.
\end{abstract}




\section{Introduction}

Clear cell renal cell carcinoma (ccRCC), the most common subtype of renal cell cancer accounting for $\sim 75 \%$ of all cases[1], is characterized by increased vascularization and an unclear clinical prognosis. Currently, patient performance status, TNM stage and Fuhrman nuclear grade are the most useful clinical available predictors of patient outcome[2]. However, interest in additional prognostic molecular markers is growing. Inactivation of the von Hippel-Lindau (VHL) gene has been shown to be a common and early event in the carcinogenesis of ccRCC[3-5]. 50 to $70 \%$ of ccRCC tumours harbour a VHL mutation [5, 6] and in 5 to $20 \%$ of ccRCCs VHL is silenced by promoter CpG island hypermethylation[7-9]. Although defective VHL functioning is a key player in sporadic and hereditary ccRCCs, tumour cell proliferation and prognosis appear not to be directly associated with alterations in the $V H L$ gene[10,11]. This finding suggests a more complex interplay of additional modifications, among which epigenetic silencing of other tumour suppressor genes may play a role.

An interesting candidate gene associated with angiogenesis[12] is the highly conserved Gremlin1 (GREM1), of which we have found an upregulated mRNA expression in a epigenome-wide screen with the demethylating agent 5'aza-2-deoxycytidine (DAC) in four cCRCC cell lines. GREM1 is a secreted glycoprotein that binds and antagonizes bone morphogenetic proteins (BMP) $-2,-4$, and -7 , thereby preventing the ability of these ligands to interact with their receptors. This results in subsequent inhibition of downstream transforming growth factor- $\beta$ (TGF- $\beta$ ) signalling [13-17]. BMPs, the largest subfamily of the TGF- $\beta$ superfamily, are pleiotropic growth factors serving multiple functions in many cell and tissue types including angiogenesis, proliferation, apoptosis, differentiation, chemotaxis, and extracellular matrix production during development as well as in adult life[18]. BMPs and BMP-antagonists such as Gremlin1, have been demonstrated in regulating renal development [19-24] and in the pathogenesis of nephropathy[25-28]. BMP-independent activities of GREM1 in cancer[14,15] and angiogenesis[12] have also been demonstrated. However, the role of GREM1 in renal cancer pathogenesis and the mechanism regulating GREM1 gene expression is not known.

GREM1 has been identified as a target of SUZ12[29], a Polycomb Repressive Complex 2 (PCR2) subunit, which is expressed during embryonic stem cell differentiation. Stem cell Polycomb group (PcG) targets are up to 12-fold more likely to have cancer-specific promoter CpG island hypermethylation[30] when compared to non-targets, suggesting epigenetic regulation of GREM1 expression in cancer. GREM1 promoter CpG island methylation has been reported in gastric-[31], bladder- and prostate cancer[32].

The aim of this study was to investigate GREM1 promoter $\mathrm{CpG}$ island methylation and its association with clinicopathological- and angiogenesis parameters in ccRCC. 


\section{Materials and methods}

\subsection{Cell lines, study populations, and clinical specimens}

Four ccRCC cell lines (SKRC1, SKRC10, SKRC52, SKRC59), kindly provided by Dr. E. Oosterwijk, Nijmegen Center for Molecular Life Sciences (NCMLS), Nijmegen, The Netherlands, were cultured in RPMI 1640 media (Invitrogen) supplemented with 10\% heat-inactivated fetal bovine serum (FBS).

Formalin-fixed and paraffin embedded tumour samples $(n=150)$ from patients with sporadic ccRCC, treated with radical or partial nephrectomy without any neo-adjuvant therapy, were collected retrospectively and further referred to as hospital-based series. Of 20 ccRCC patients also histologically normal renal tissue was available. In addition, histologically normal renal tissue samples (formalin-fixed, paraffin embedded) of 18 non-RCC patients was collected. All samples were derived from the archives of the Department of Histopathology, University Hospital of Leuven and Department of Pathology, Maastricht University Medical Center. Hematoxylin eosin-stained slides were revised by an experienced genitourinary pathologist to confirm nuclear Fuhrman grading. Tissues were sectioned into $20 \mu \mathrm{m}$ slides and genomic DNA was isolated and purified using the Puregene DNA purification kit according to the manufacturers instructions (BIOzym, Landgraaf, The Netherlands). Data of postoperative follow-up (median follow-up 64 months, range 1-153 months) at regular intervals by means of physical examination, chest X-ray, abdominal computed tomography or ultrasound, and when indicated a bone scan, was available (for patient characteristics see table $1 \mathrm{~A}$ ).

A second independent, population-based validation series of formalin-fixed, paraffin embedded ccRCC samples $(n=185)$ was obtained from the Netherlands Cohort Study on diet and cancer (NLCS)[33] and collected from 51 pathology laboratories throughout the Netherlands with a median follow-up period of 79 months, range 0-218 months. This series will be further referred to as population-based series. Tissue collection and DNA isolation have been described in detail elsewhere[34]. Hematoxylin eosin-stained slides were revised by an experienced genitourinary pathologist. Information on tumour size and stage was available from the pathological reports and cancer registries. Follow-up was accomplished by record linkage to the municipal population registry and the causes of death registry from Statistics Netherlands[11] (for patient characteristics see table 1B). This study was approved by the Medical Ethical Committee of the Maastricht University Medical Center.

\subsection{Drug treatment and gene expression analysis}

cCRCC cell lines SKRC1, SKRC10, SKRC52, and SKRC59 were treated with 5'aza-2deoxycytidine (DAC) or Trichostatin A (TSA), which inhibit DNA methylation and histone deacetylases, respectively. $10 \%$ confluent RCC cells were cultured in RPMI 1640 media containing 10\% FBS with $5 \mu \mathrm{M}$ (DAC) (Sigma; stock solution: $1 \mathrm{mM}$ in PBS) for 96 hours, replacing media and DAC every 24 hours. Cell treatment with 300nM TSA 
(Sigma; stock solution: $5 \mathrm{mM}$ dissolved in ethanol) was performed for 18 hours, starting at $\sim 30 \%$ cell confluency. Mock treated cells were grown in parallel with the DAC treatment by addition of equal volumes of PBS without drugs.

Table 1.A: GREM1 promoter CpG island methylation associated with clinical, histopathological, and angiogenesis-related patient characteristics (hospital-based series)

\begin{tabular}{|c|c|c|c|c|}
\hline & $\begin{array}{c}\text { All ccRCC } \\
n=150(100 \%)\end{array}$ & $\begin{array}{c}\text { Methylated } \\
\text { GREM1-region i } \\
\mathrm{n}=77(55 \%)\end{array}$ & $\begin{array}{c}\text { Methylated } \\
\text { GREM1-region ii } \\
\mathrm{n}=33(24 \%)\end{array}$ & $\begin{array}{c}\text { Methylated } \\
\text { GREM1-region iii } \\
\mathrm{n}=28(20 \%)\end{array}$ \\
\hline Sex & & & & \\
\hline Male & 99 (66\%) & $50(65 \%)$ & $22(67 \%)$ & 22 (79\%) \\
\hline Female & $51(34 \%)$ & $27(35 \%)$ & $11(33 \%)$ & $6(21 \%)$ \\
\hline $\mathrm{P}(\mathrm{U}$ versus $\mathrm{M})$ & & 0.38 & 0.95 & 0.11 \\
\hline Age (mean) & $60.1( \pm 12.1 \mathrm{SD})$ & $60.3 b( \pm 12.6 \mathrm{SD})$ & $62.3( \pm 11.6 \mathrm{SD})$ & $60.3( \pm 10.4$ SD $)$ \\
\hline$P(U$ versus $M)$ & & 0.80 & 0.33 & 0.96 \\
\hline Tumour size in $\mathrm{cm}$ (mean) & $6.36( \pm 3.45 \mathrm{SD})$ & $6.91( \pm 3.55$ SD) & $7.25( \pm 3.54 \mathrm{SD})$ & 7.72 ( $\pm 3.17 \mathrm{SD})$ \\
\hline$P(U$ versus $M)$ & & 0.01 & 0.08 & 0.02 \\
\hline Fuhrman grade 1 & $15(10 \%)$ & $5(6 \%)$ & $2(6 \%)$ & $1(4 \%)$ \\
\hline 2 & $72(48 \%)$ & $36(47 \%)$ & $15(46 \%)$ & $11(39 \%)$ \\
\hline 3 & $46(31 \%)$ & $26(34 \%)$ & $10(30 \%)$ & $9(32 \%)$ \\
\hline 4 & $17(11 \%)$ & $10(13 \%)$ & $6(18 \%)$ & $7(25 \%)$ \\
\hline$P(U$ versus $M)$ & & 0.54 & 0.61 & 0.04 \\
\hline Tumour stage & $8(5 \%)$ & $3(4 \%)$ & $0(0 \%)$ & $0(0 \%)$ \\
\hline 2 & $89(60 \%)$ & $42(54 \%)$ & $17(53 \%)$ & $12(43 \%)$ \\
\hline 3 & $30(20 \%)$ & $16(21 \%)$ & $9(28 \%)$ & $7(25 \%)$ \\
\hline 4 & $22(15 \%)$ & $16(21 \%)$ & $6(19 \%)$ & $9(32 \%)$ \\
\hline$P(U$ versus $M)$ & & 0.23 & 0.32 & 0.013 \\
\hline ECP \% (mean) & $1.4( \pm 2.17 S D)$ & $1.8( \pm 2.72 \mathrm{SD})$ & $2.5( \pm 3.37 \mathrm{SD})$ & $3.0( \pm 2.97 \mathrm{SD})$ \\
\hline$P(U$ versus $M)$ & & 0.097 & 0.004 & 0.0001 \\
\hline TCP \% (mean) & $6.7( \pm 9.25 \mathrm{SD})$ & $8.2( \pm 11.40 \mathrm{SD})$ & 9.0 ( $\pm 12.70 \mathrm{SD})$ & $12.0( \pm 11.90 \mathrm{SD})$ \\
\hline$P(U$ versus $M)$ & & 0.13 & 0.16 & 0.001 \\
\hline MVD per $\mathrm{mm}^{2}$ (mean) & $197.5( \pm 91.0 \mathrm{SD})$ & $177.4( \pm 81.70$ SD) & $174.8( \pm 89.20$ SD $)$ & $152.5( \pm 92.2 \mathrm{SD})$ \\
\hline$P(U$ versus $M)$ & & 0.01 & 0.09 & 0.001 \\
\hline
\end{tabular}

Tumour stage based on International Union against Cancer (UICC) TNM classification of malignant tumours, fourth, fully revised edition, 1987; P: p-value for comparison of methylated and unmethylated cases; ECP: endothelial cell proliferation; TCP: tumour cell proliferation; MVD: microvessel density.

RNA of mock-, DAC-, and TSA-treated cells was isolated using the RNeasy kit (Qiagen) according to the manufacturers' instructions. For real time RT-PCR, $1 \mu \mathrm{g}$ of total RNA was reverse transcribed by using Ready-To-Go ${ }^{\mathrm{TM}}$ You-Prime First-Strand Beads (Amersham Biosciences/ GE Health care) with addition of random hexamers ( $0.2 \mu \mathrm{g}$ per reaction). (Primer sequences and PCR conditions are provided in supplemental table S1). 
Table 1.B: GREM1 promoter CPG island methylation associated with clinical, histopathological, and angiogenesis-related patient characteristics (population-based series)

\begin{tabular}{|c|c|c|}
\hline & $\begin{array}{c}\text { All ccRCC } \\
n=185(100 \%)\end{array}$ & $\begin{array}{c}\text { Methylated } \\
\text { GREM1-region iii } \\
\mathrm{n}=68(40 \%)\end{array}$ \\
\hline \multicolumn{3}{|l|}{ Sex } \\
\hline Male & $108(59 \%)$ & $44 \quad(65 \%)$ \\
\hline Female & 74 (41\%) & $24 \quad(35 \%)$ \\
\hline $\mathrm{P}(\mathrm{U}$ versus $\mathrm{M})$ & & 0.32 \\
\hline Age (mean) & 67.4 ( $\pm 4.7 \mathrm{SD})$ & $67.2( \pm 5.2 \mathrm{SD})$ \\
\hline $\mathrm{P}(\mathrm{U}$ versus $\mathrm{M})$ & & 0.49 \\
\hline Tumour size in cm (mean) & $7.9 \quad( \pm 3.9 \mathrm{SD})$ & $7.5 \quad( \pm 3.17 \mathrm{SD})$ \\
\hline$P(U$ versus $M)$ & & 0.16 \\
\hline Fuhrman grade 1 & $42 \quad(23 \%)$ & 10 (15\%) \\
\hline 2 & $66 \quad(36 \%)$ & 22 (32\%) \\
\hline 3 & $47 \quad(26 \%)$ & 23 (34\%) \\
\hline 4 & $27 \quad(15 \%)$ & 13 (19\%) \\
\hline $\mathrm{P}(\mathrm{U}$ versus $\mathrm{M})$ & & 0.03 \\
\hline Tumour stage 1 & $4 \quad(2 \%)$ & $0 \quad(0 \%)$ \\
\hline 2 & $81 \quad(45 \%)$ & 27 (40\%) \\
\hline 3 & $63 \quad(35 \%)$ & 23 (34\%) \\
\hline 4 & 32 (18\%) & 18 (26\%) \\
\hline $\mathrm{P}(\mathrm{U}$ versus $\mathrm{M})$ & & 0.08 \\
\hline
\end{tabular}

Tumour stage based on International Union against Cancer (UICC) TNM classification of malignant tumours, fourth, fully revised edition, 1987; P: p-value for comparison of methylated and unmethylated cases; ECP: endothelial cell proliferation; TCP: tumour cell proliferation; MVD: micro vessel density.

\subsection{GREM1 methylation-specific PCR and bisulfite sequencing}

The highly conserved human GREM1 gene (accession number NM_013372) is located on chromosome 15q13-q15. For CpG island prediction, EMBOSS

(http://bioinfo.hku.hk/EMBOSS) software using the following criteria (Obs/Exp: 0.6, MinPC: 50, length: 200) was used. This resulted in three closely located $\mathrm{CpG}$ islands from -650 bp to +1671 bp relative to the transcription start site (TSS) (figure 1A), which will be further considered as one island. To cover a large part of the promoter region for GREM1 methylation analysis, three different nested methylation-specific PCR (MSP) primer sets (GREM1-region i, -region ii, and -region iii) were designed (see figure 1A). GREM1 promoter CpG island methylation was determined by chemical modification of genomic DNA with sodium bisulfite and subsequent MSP as described in detail elsewhere[35-37]. In brief, 500 ng of DNA was modified by sodium bisulfite using the EZ DNA methylation kit (Zymo Research, Orange, California) according to the manufacturer's instruction. To facilitate MSP analysis on DNA retrieved from formalin-fixed paraffin embedded tissue, DNA was first amplified with flanking PCR primers that amplify bisulfite-modified DNA but do not preferentially amplify methylated or unmethylated DNA. The resulting fragment was used as a template for the MSP reaction. GREM1-region i, -region ii, -region iii methylation was analyzed on the hospital-based series of ccRCCS. On the population-based series, GREM1-region iii MSP was performed. All PCRs were performed with controls for unmethylated alleles (DNA from normal lymphocytes or DNA from human umbilical vein endothelial cells (HUVEC), 
methylated alleles [normal lymphocyte DNA treated in vitro with Sssl methyltransferase (New England Biolabs)], and a control without DNA. Primer sequences and PCR conditions are provided in supplemental table S1. Ten $\mu$ of each MSP reaction was directly loaded onto $2 \%$ agarose gels containing GelStar Nucleic Acid Gel Stain (Cambrex, New Jersey, USA), and visualized under UV illumination. The methylation status of the hospital-based ccRCC series was successfully obtained in 93-95\% (138-141 of 149 , one sample failed to provide a sufficient amount of DNA), dependent on the primer set used, and in 91\% (168 of 185) of the population-based series. To assess reproducibility, MSP reactions have been performed in duplicate or triplicate starting from DNA amplification with flanking PCR primers. The reproducibility was $82 \%, 95 \%$, and $97 \%$ for primer set $\mathrm{i}$, ii, and iii, respectively in the hospital-based series and $84 \%$ in the population-based series. Non-concordant MSP results were analyzed a third time and a two out of three concordance was used as end result.

For bisulfite sequencing, PCR products were cloned using the TOPO TA cloning kit (Invitrogen, Breda, the Netherlands), single colonies were sequenced using M13 primer, BigDye Terminator v1.1 Cycle Sequencing Kit in accordance to the manufacturer's instruction. Primer sequences and PCR conditions are provided in supplemental table S1.

\subsection{Assessment of angiogenesis and tumour parameters}

Quantification of microvessel density (MVD), endothelial cell proliferation (ECP), and tumour cell proliferation (TCP) in the hospital-based series has been described in detail elsewhere[38].

Briefly, CD34/Ki67 double immunohistochemical staining[39] was used to analyze MVD, ECP and TCP. One tissue slide was analyzed per tumour. For assessment of the vascular density within a tumour tissue slide, 1 hotspot (most vascularized microscopic field) was selected and 4 areas were chosen randomly. Vessel counts were performed at 200x magnification using an optical grid. The presence of a vascular lumen was not necessary to identify a microvessel. MVD was expressed as vessels per $\mathrm{mm}^{2}$. Next, a total number of at least 500 intratumoural endothelial cells and 500 tumour cells were evaluated in consecutive fields at a 400x magnification and the fractions of proliferating endothelial cells (ECP\%) and tumour cells (TCP\%) were assessed. ECP\% and TCP\% were calculated according to the following formulas: $\mathrm{ECP} \%$ = (the number of endothelial cells with Ki67-stained nuclei/total number of endothelial cells evaluated) x 100; $\mathrm{TCP} \%$ = (the number of tumour cells with Ki67-stained nuclei/total number of tumour cells evaluated) $\times 100$.

\subsection{Statistical analysis}

Cause-specific survival was defined as the time from cancer diagnosis until renal cancer-related death or until the end of follow-up. Differences in clinicopathological and angiogenesis characteristics between ccRCCs with and without GREM1 promoter CpG 
island methylation were evaluated with the Student's t-tests and Pearsons $\chi^{2}$ tests. Kaplan-Meier analyses and log-rank tests were used to estimate the overall influence of GREM1 promoter CpG island methylation on cause-specific survival. Hazard ratios (HR) and corresponding 95\% confidence interval (Cl) were assessed by use of Cox proportional hazard models. Factors were considered possible confounders if they were known prognostic factors for renal cancer and influenced the crude HR. Possible confounders that were included in the model for both series were sex, age at diagnosis, cancer stage, tumour size and nuclear grade. The proportional hazard assumption was tested using the Schoenfeld residuals. Analyses were performed by use of the statistical package STATA 9.0. All reported $P$ values are two-sided and $P$ values $\leq 0.05$ were considered statistically significant. 
Prognostic significance of Gremlin1

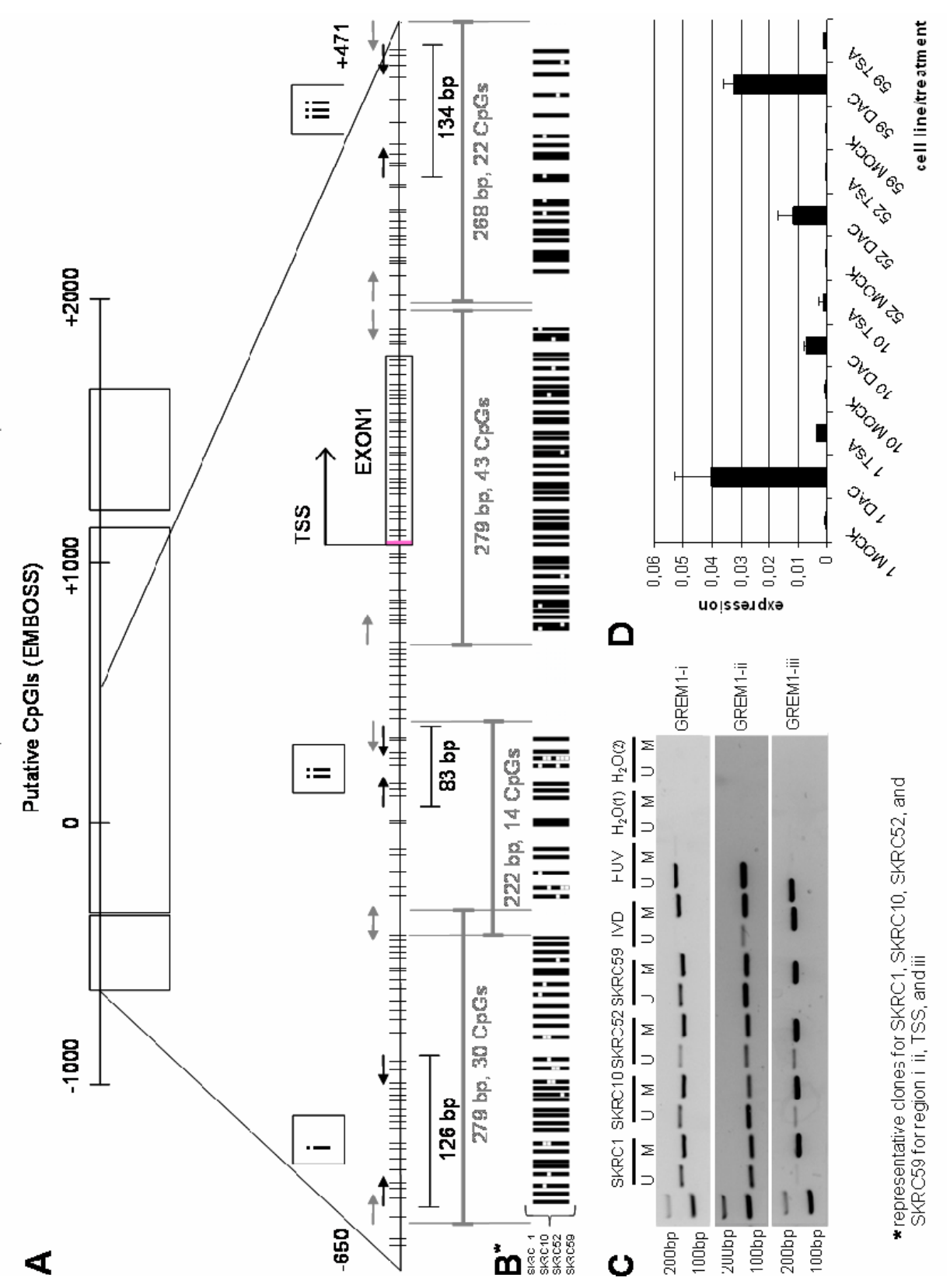

Figure 1 


\section{Results}

\subsection{Promoter CPG island methylation and downregulation of GREM1 mRNA expression in ccRCC cell lines}

GREM1 promoter CpG island methylation and mRNA expression were analyzed by MSP and real time RT-PCR in the CCRCC cell lines SKRC1, SKRC10, SKRC52, SKRC59. All four cell lines were heavily methylated at region $\mathrm{i}$, ii and iii of the CpG island (figure 1C). MSP data were confirmed by bisulfite sequencing of region i ( $279 \mathrm{bp}, 30 \mathrm{CpGs}$ ), ii 222 bp, 14 CpGs) and region iii (268 bp, 22 CpGs). Methylation at the TSS (279 bp, 43 CpGs) was assessed by bisulfite sequencing (figure 1B). As shown in figure 1D, little or no mRNA expression is observed in SKRC1, SKRC10, SKRC52, and SKRC59. mRNA expression could be induced in all four cell lines by DAC but not, or to a much lesser extent, by TSA.

\subsection{GREM1 promoter CpG island hypermethylation in cCRCCs varies at different regions in the promoter $C p G$ island}

To analyse GREM1 promoter CpG island methylation in primary ccRCCs, a hospitalbased series of ccRCCs was analyzed for methylation at three loci in the promoter. $63 \%$ (93/147, 3 samples did not yield a MSP result in region i, ii and iii) of the ccRCCs showed methylation of one or more of the three analyzed regions. A decrease of methylation prevalence is seen more downstream in the analyzed area, with $55 \%$ (77/141) methylation for region i (-648 - -462), 24\% (33/139) for region ii (-236 - -140), and $20 \%(28 / 138)$ for region iii $(+311-+472)$ (Table $1 \mathrm{~A})$. Methylation in matching histologically normal renal tissues was observed in region i $(50 \%, 10 / 20)$, while lower methylation frequencies were observed in region ii and iii $(5 \%, 1 / 20$ and $10 \%, 2 / 20$, respectively), data not shown. In histologically normal renal tissues from non-cancer patients, $0-6 \%$ methylation was observed in the subregions (i: $1 / 18$, ii:1/18, iii: 0/18) (data not shown).

\subsection{GREM1 promoter CPG island methylation at region iii is associated with increased tumour size, tumour grade and -stage, angiogenesis and poor prognosis}

Next, we studied the association of GREM1 promoter CpG island methylation and patient characteristics and clinical outcome. In table $1 \mathrm{~A}$, patient and tumour characteristics for the 150 cCRCC cases included in the GREM1 promoter hypermethylation analyses are presented; methylated cases were stratified by methylation site. No differences among methylated and unmethylated cases were observed in the distribution of age and sex ( $p$-values $>0.05$, see table $1 \mathrm{~A}$ ). Consistent with the number of methylated cases, associations between GREM1 promoter methylation and clinical-, histopathological- and angiogenesis related patient characteristics are dependent on the region of methylation. GREM1-region i methylation was associated with tumour size $(p=0.01)$ and MVD ( $p=0.01)$. The median OS and MFS for unmethylated versus methy- 
lated cases at regio $i$ is 60.5 (1-143) versus 64.5 (range 2-153) and 58.5 (1-143) versus 41 months (0-143), respectively. The Kaplan-Meier curve for GREM1-region i methylation (Figure 2A) shows a borderline statistically significant difference in OS for methylated and unmethylated cases (log-rank, $p=0.05$ log-rank MFS, $p=0.73$, data not shown). Age and sex adjusted Cox proportional hazard analyses show a statistically significant association between GREM1-region i methylation and $\mathrm{OS}(\mathrm{HRi}=1.84 ; 95 \%$ $\mathrm{Cl}, 1.02-3.32$, but not for $\mathrm{MFS}(\mathrm{HRi}=1.08 ; 95 \% \mathrm{Cl}, 0.76-1.54)$. Multivariate analyses however, show no association between GREM1-region $\mathrm{i}$ and OS or MFS ( $\mathrm{HRi}=0.66$; 95\% $\mathrm{Cl}, 0.33-1.33$, and $\mathrm{HRi}=0.82 ; 95 \% \mathrm{Cl}, 0.55-1.21$, respectively)

GREM1-region ii methylation was only significantly associated with ECP $(p=0.004)$. The median OS and MFS for unmethylated versus methylated cases at regio ii is 60.5 (1153) versus 76 (range 1-153) and 56 (1-153) versus 60 months (0-153), respectively. Figure 2B shows the Kaplan-Meier curve for GREM1-region ii; no difference was observed between methylated and unmethylated cases (log-rank $p=0.84$, log-rank MFS, $\mathrm{p}=0.15$, data not shown). Methylation of GREM1-region ii was not significantly associated with OS or MFS in the age and sex adjusted Cox regression analyses (HR(OS)ii= 0.93; $95 \% \mathrm{Cl}, 0.48-1.80$, and $\mathrm{HR}(\mathrm{MFS}) \mathrm{ii}=0.74 ; 95 \% \mathrm{Cl}, 0.49-1.12)$ nor in the multivariate analyses ( $\mathrm{HR}(\mathrm{OS}) \mathrm{ii}=0.55 ; 95 \% \mathrm{Cl}, 0.26-1.18$, and $\mathrm{HR}(\mathrm{MFS}) \mathrm{ii}=0.56 ; 95 \% \mathrm{Cl}, 0.36-0.87)$. In contrast, methylated ccRCCs at region iii are statistically significant larger in tumour size $(p=0.02)$, and have a statistically significant higher tumour grade $(p=0.04)$, tumour stage $(p=0.013)$, ECP ratio $(p=0.0001)$, and TCP ratio $(p=0.001)$, as compared to unmethylated tumours at region iii, and methylated cases have a lower MVD (per mm2, $\mathrm{p}=0.001$ ). The median OS and MFS for unmethylated versus methylated cases at region iii is 65.5 (1-153) versus 56 (range 2-138) and 60 (1-153) versus 23 months (0-138), respectively. Figure $2 \mathrm{C}$ shows the Kaplan-Meier curve for cause-specific survival and GREM1-region iii methylation, and methylated cases had a statistically significant worse survival as compared to unmethylated cases (log-rank $p=0.002$, log-rank MFS $p=0.05$, data not shown). Age and sex adjusted Cox proportional hazard analyses also show a significant association between GREM1-region iii methylation and OS (HR= 2.35; 95\% Cl 1.29-4.28); the association with MFS was borderline significant ( $\mathrm{HR}=1.54$; 95\% $\mathrm{Cl}$ 0.99-2.39). However, in multivariate analyses adjusted for age, sex, grade, stage, and tumour size, GREM1-region iii methylation does not seem to be an independent prognostic factor: $\mathrm{HR}(\mathrm{OS})=0.88,95 \% \mathrm{Cl}: 0.45-1.74$, and $\mathrm{HR}(\mathrm{MFS})=1.04,95 \% \mathrm{Cl}$ : $0.65-1.68$

Due to the greatest cancer specificity of GREM1 promoter CpG island methylation at region iii, we wanted to validate the results obtained for the GREM1-region iii region, in an independent population-based series of ccRCC (table1B). Age and sex were equally distributed among the methylated and unmethylated cases in this series, ( $P$ values $>0.05$, see table $1 B$ ). $40 \%$ (68 of 168 ) of the cCRCCS with MSP results were methylated and methylated tumours more often had a higher tumour grade $(p=0.03)$ as compared to unmethylated tumours. A similar trend was seen for tumour stage 
$(p=0.08)$. The median OS for unmethylated versus methylated cases at region iii is 96 (1-218) versus 42 (range 1-212), respectively. Figure 2D shows the Kaplan-Meier curve for cause-specific survival and GREM1-region iii methylation, and methylated cases had a statistically significant worse survival as compared to unmethylated cases (logrank test $\mathrm{p}=0.0001)$. Cox regression analyses also showed a statistically significant association between methylation and OS with an age and sex adjusted HR of 2.32 (95\% $\mathrm{Cl}: 1.52-3.53)$ and a multivariate $\mathrm{HR}$ of 2.27 (95\% Cl: $1.44-3.59$, adjusted for age and sex, grade, stage and size).
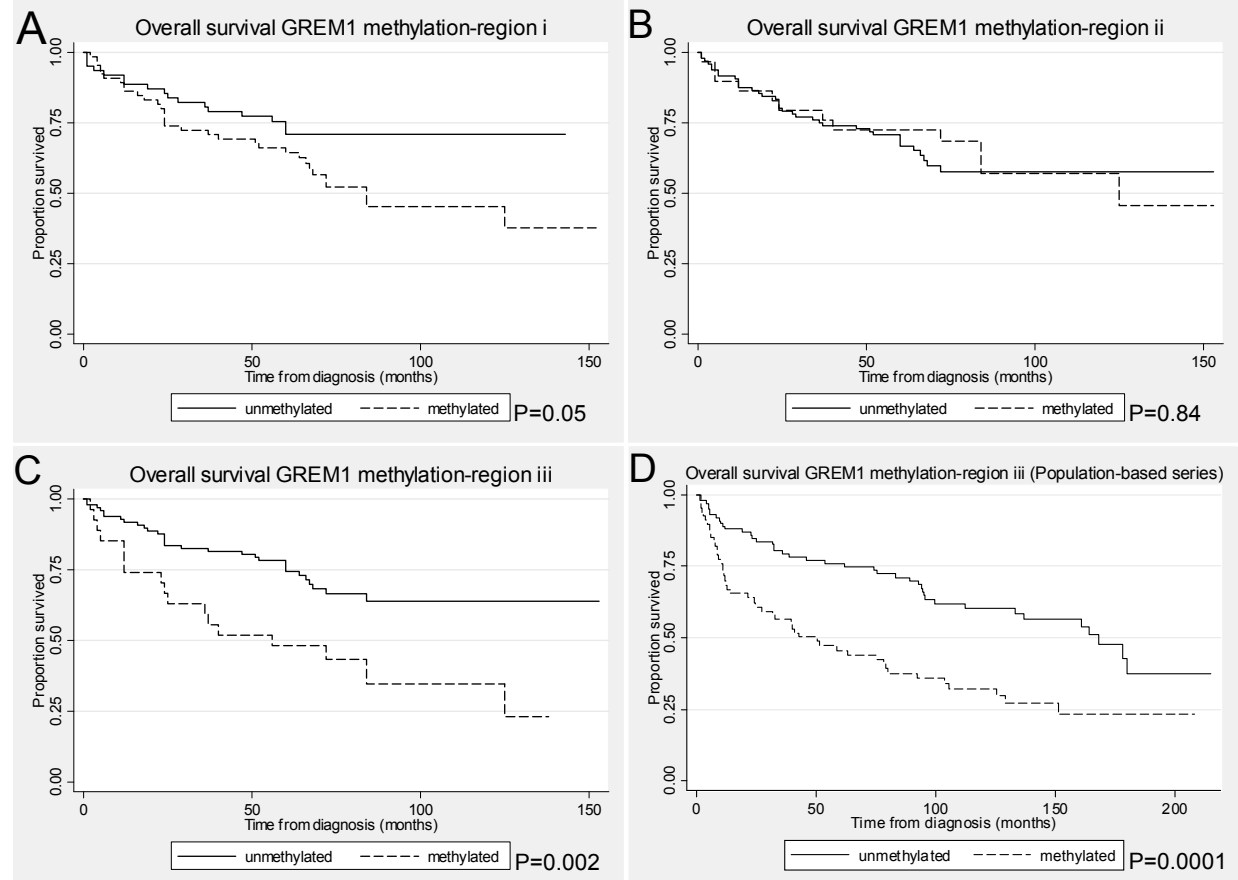

Figure 2. Overall cause-specific survival curves for ccRCC patients according to GREM1 promoter CpG island methylation at region $\mathrm{i}(\mathrm{A})$, ii (B), and iii (C, D), with $A, B$, and $C$ for patients of the hospital-based series and $D$ for patients of the population-based series.

\section{Discussion}

Epigenetic silencing of GREM1 by promoter $\mathrm{CpG}$ island methylation is present in ccRCC cell lines, and can be reversed by inhibiting DNA methyltransferases, indicating that DNA methylation is involved in GREM1 silencing. We have also shown an association between GREM1 promoter CPG island hypermethylation and increased malignancy/angiogenesis in primary ccRCCs, while methylation in histologically normal renal tissue is an infrequent phenomenon. Interestingly, GREM1 promoter CpG island methylation in primary ccRCCs varies within the CpG island, with frequencies of $55 \%, 24 \%$, 
and $20 \%$ for region $\mathrm{i}$, ii, and $\mathrm{iii}$, respectively. We showed that within one $\mathrm{CpG}$ island, CPG methylation at loci only a few hundred base pairs apart can differ strongly. As previously shown for $h M L H 1$ in colorectal cancer, methylation associated transcriptional silencing can occur in a region-specific manner[40], indicating that also clinical effects may be methylation-region specific.This observation emphasizes that the location of $\mathrm{CpG}$ dinucleotides to be analyzed and with this, MSP primer location and design, can influence the observed methylation prevalence and clinical association[41]. Analyses of clinicopathological characteristics revealed that significant differences between GREM1 methylated and unmethylated ccRCCS are also dependent on the region analyzed. Only for the most downstream region (iii), $\mathrm{CpG}$ methylation was statistically significant associated with increased malignancy, i.e.larger tumours, higher tumour grade and -stage, and a worse prognosis. These data suggest that the effect of GREM1 promoter CPG island methylation on CCRCC biology depends on the region methylated within the $\mathrm{CpG}$ island. Whether GREM1 promoter $\mathrm{CPG}$ island methylation initiates at region $i$ and than spreads downstream towards the TSS and region iii or starts in region iii and than retroverts towards region $i$ can not be deduced from these data.

The second series is derived from the population-based prospective Netherlands Cohort Study, and therefore appropriate for validation of the results. This series confirmed the associations between region iii methylation and tumour grade and prognosis which were found in the hospital-based series. In addition, this validation points to GREM1 as a gene independently influencing the progression of ccRCCs when methylated at region iii (figure $3 \mathrm{~B}$ ), which was not found in the hospital-based series to the same extent (figure 3A). Possible differences between the two ccRCC series were not apparent, but could perhaps account for this observation. Nevertheless, both series do indicate a significant effect of GREM1-region iii methylation on increased malignant behaviour and a worse prognosis of ccRCC. 


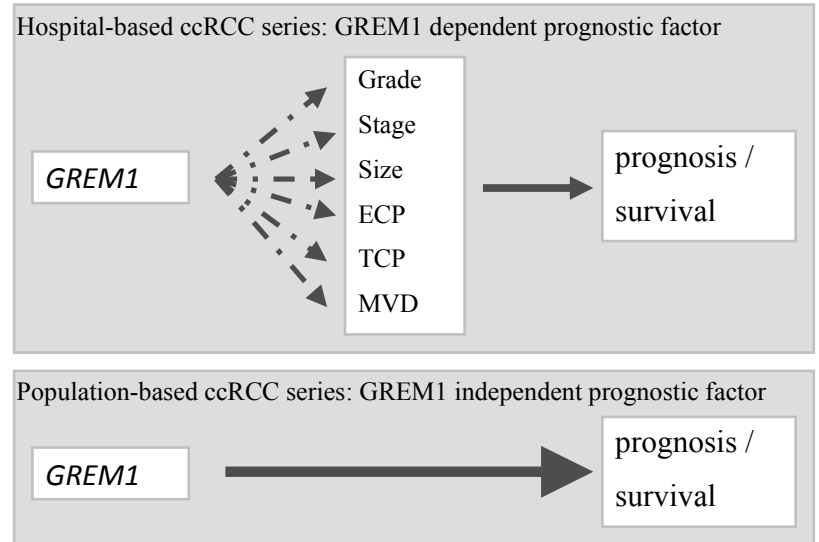

Figure 3. Prognostic significance of GREM1 promoter CpG island methylation in hospital-based and population-based ccRCC patient series

Our data also show that GREM1-region iii methylation is significantly associated with increased ECP, TCP and lower MVD, suggesting that GREM1 silencing promotes active angiogenesis, pointing to GREM1 as an anti-angiogenesis factor. Similar observations showing that BMP2 and BMP4 exert angiogenic activity, which may increase with inactivation of the BMP-antagonist GREM1 have been reported[42-44]. However, a recent study by Stabile et al. indicated a BMP-independent, pro-angiogenic function of GREM1 by showing GREM1 expression in human lung tumour endothelium and binding of GREM1 to the endothelial cell surface thereby activating intracellular signalling and cell motility[12]. An angiogenic role of GREM1 may be tissue specific and/or dosedependent. ECP also may be indirectly influenced by GREM1 through its strong correlation with TCP (Rho=0.82, $\mathrm{p}<0.001$ ). Moreover, during tumour progression, cumulative genetic/epigenetic events can activate additional angiogenic growth factors, resulting in an increased tumour driven angiogenesis. Additionally, GREM1 promoter CpG island methylation may affect maturation of intratumoural blood vessels in ccRCC, as Maciel et al. reported induction of vascular smooth muscle cell (VSMC) proliferation and migration by Gremlin[45]. This is supported by the fact that cCRCC with high ECP, representing high grade ccRCC, are shown to be characterized by a less stabilized, less mature vasculature[38]. Therefore, GREM1 inactivation may impede tumour vessel maturation, as observed in high grade tumours, in which less differentiated vessels provide an easier access for cancer cells to the circulation.

Except for sclerostin domain-containing-1 (SOSTDC1), another BMP antagonist which suppresses proliferation of RCC cells and is downregulated in RCC[46], no other BMP antagonists have previously been implicated in RCC. GREM1 has been shown to be down regulated in several tumour cell lines i.e. neuroblastoma, fibrosarcoma, colon 
adenocarcinoma, breast carcinoma, suggesting a tumour suppressor function[14,47]. However, the role of GREM1 in renal carcinogenesis has not yet been elucidated. Silencing of GREM1 by promoter $\mathrm{CpG}$ island methylation may play a role in carcinogenesis both by affecting cell cycle and by upregulation of BMP signalling. Earlier studies have linked BMPs and BMP receptors (BMP-RI and BMP-RII) to cancer[48-50]. Biological responses to BMP are cell type-specific and divergent effects with both oncogenic and tumour suppressor activities have been described[49]. Despite the progress achieved in delineating the functional significance of BMP pathways in carcinogenesis in general during the last decade[51-57], little is known about the importance of BMP signalling in RCC.

In conclusion, our results indicate that GREM1 could act as a tumour suppressor gene in CCRCC, and that DNA promoter methylation of GREM1 has an impact on the biology and outcome of this type of cancer. Notably, GREM1-region iii promoter $\mathrm{CpG}$ island hypermethylation appears to be independently associated with aggressive tumour behaviour and increased angiogenesis and therefore could be an interesting prognostic biomarker. Probably, this prognostic effect is mediated through an increase TCP and ECP. However, the exact mechanism of GREM1 inactivation and its relation to tumour progression as well as its role in angiogenesis need to be further clarified. In addition, further studies are needed to explore whether GREM1 promoter CpG island methylation can also be detected in urine or serum samples for use as a possible noninvasive marker for aggressive ccRCC.

\begin{tabular}{|c|c|c|c|c|c|c|}
\hline method & & Forward $5^{\prime}>3^{\prime}$ & Reverse $5^{\prime}>3^{\prime}$ & $\begin{array}{l}\mathrm{Tm} \\
\left({ }^{\circ} \mathrm{C}\right)\end{array}$ & $\begin{array}{l}\text { cycles } \\
\text { (n) }\end{array}$ & $\begin{array}{l}\text { length } \\
\text { (bp) }\end{array}$ \\
\hline RT & & CAGCCTACACGGTGGGAGC & CTGCTCTGAGTCATTGTGCTGG & & & 128 \\
\hline \multirow[t]{5}{*}{ MSP i } & $\mathrm{FI}$ & TGGYGATAGGTTTTTGTTGG & CCCCTCTAAAAAAAACRAAACTA & 56 & 35 & 156 \\
\hline & u & GTTTTTGTTGGTGGTTAGTGAGTT & AACAAAACTACAAAATACTCTAAT & & & \\
\hline & & $\mathrm{T}$ & CAAACA & 62 & 30 & 134 \\
\hline & M & TGTTGGCGGTTAGCGAGTTC & GAAACTACGAAATACTCTAATCGA & & & \\
\hline & & & ACG & 62 & 30 & 126 \\
\hline \multirow[t]{4}{*}{ MSP ii } & $\mathrm{FI}$ & TTTTGGGGTGATTTTTTTTAGT & САССССТССТАСТТСССC & 56 & 35 & 96 \\
\hline & $\mathrm{u}$ & GTGATTTTTTTTAGTTGGTTTTGGG & АССССТССТАСТТССССАСА & & & \\
\hline & & $\mathrm{T}$ & & 64 & 35 & 88 \\
\hline & M & ATTTTTTTTAGTCGGTTCGGGC & CCCTCCTACTTCCCCGCG & 64 & 35 & 83 \\
\hline \multirow[t]{3}{*}{ MSP iii } & $\mathrm{FI}$ & GGGGTTTTTTTTGTTGAGGT & СТАCTACCACCAACACCAAAAAC & 56 & 35 & 161 \\
\hline & $\mathrm{U}$ & TTTTTTTGTTGAGGTTGTGGATATT & TCAAAAACATATTCAAAAACCTCCA & 65 & 25 & 140 \\
\hline & M & TTTGTTGAGGTCGCGGATATC & AAAACGTATTCGAAAACCTCCG & 65 & 25 & 134 \\
\hline BSseq i & & TGGYGATAGGTTTTTGTTGG & CCCAAATCTAAACTTTCCCTATC & 60 & 35 & 279 \\
\hline BSseq ii & & GATAGGGAAAGTTTAGATTTGGG & САССССТССТАСТTСССC & 64 & 35 & 222 \\
\hline BSseq iii & & AGGGTYGTAAATTAATTTAGGATT & CTACTACCACCAACACCAAAAAC & 60 & 40 & 268 \\
\hline BSseq TSS & & GGYGTTTGGTTAATGGAGAGG & CRCCCACTCACATCCCTACC & 62 & 35 & 279 \\
\hline
\end{tabular}




\section{References}

1. Kovacs G, Akhtar M, Beckwith BJ, et al. (1997) The Heidelberg classification of renal cell tumours. $J$ Pathol 183,131-3.

2. Gelb AB. (1997) Renal cell carcinoma: current prognostic factors. Union Internationale Contre le Cancer (UICC) and the American Joint Committee on Cancer (AJCC). Cancer 80,981-6.

3. Kaelin WG, Jr. (2004) The von Hippel-Lindau tumor suppressor gene and kidney cancer. Clin Cancer Res $10,6290 \mathrm{~S}-5 \mathrm{~S}$.

4. van Dijk BA, Schouten L, Oosterwijk E, et al. Cigarette smoking, von Hippel-Lindau gene mutations and sporadic renal cell carcinoma. (2006) Br J Cancer 95,374-7.

5. Gnarra JR, Duan DR, Weng Y, et al. (1996) Molecular cloning of the von Hippel-Lindau tumor suppressor gene and its role in renal carcinoma. Biochim Biophys Acta 1242,201-10.

6. Richards FM. (2001) Molecular pathology of von HippelLindau disease and the VHL tumour suppressor gene. Expert Rev Mol Med 2001,1-27.

7. Banks RE, Tirukonda P, Taylor C, et al. (2006) Genetic and epigenetic analysis of von Hippel-Lindau (VHL) gene alterations and relationship with clinical variables in sporadic renal cancer. Cancer Res 66,200011.

8. Dulaimi E, Ibanez de Caceres I, Uzzo RG, et al. (2004) Promoter hypermethylation profile of kidney cancer. Clin Cancer Res 10,3972-9.

9. Herman JG, Latif F, Weng Y, et al. (1994) Silencing of the VHL tumor-suppressor gene by DNA methylation in renal carcinoma. Proc Natl Acad Sci U S A 91,9700-4.

10. Schraml P, Struckmann K, Hatz F, et al. (2002) VHL mutations and their correlation with tumour cell proliferation, microvessel density, and patient prognosis in clear cell renal cell carcinoma. $J$ Pathol 196,186-93.

11. Smits KM, Schouten LJ, van Dijk BA, et al. (2008) Genetic and epigenetic alterations in the von hippellindau gene: the influence on renal cancer prognosis. Clin Cancer Res 14,782-7.

12. Stabile H, Mitola S, Moroni E, et al. (2007) Bone morphogenic protein antagonist Drm/gremlin is a novel proangiogenic factor. Blood 109,1834-40.

13. Hsu DR, Economides AN, Wang X, Eimon PM, Harland RM. (1998) The Xenopus dorsalizing factor Gremlin identifies a novel family of secreted proteins that antagonize BMP activities. Mol Cell 1,673-83.

14. Topol LZ, Marx M, Laugier D, et al. (1997) Identification of drm, a novel gene whose expression is suppressed in transformed cells and which can inhibit growth of normal but not transformed cells in culture. Mol Cell Biol 17,4801-10.

15. Topol LZ, Modi WS, Koochekpour S, Blair DG. (2000) DRM/GREMLIN (CKTSF1B1) maps to human chromosome 15 and is highly expressed in adult and fetal brain. Cytogenet Cell Genet 89,79-84.

16. Topol LZ, Bardot B, Zhang Q, et al. (2000) Biosynthesis, post-translation modification, and functional characterization of Drm/Gremlin. J Biol Chem 275,8785-93.

17. Merino R, Rodriguez-Leon J, Macias D, Ganan Y, Economides AN, Hurle JM. (1999) The BMP antagonist Gremlin regulates outgrowth, chondrogenesis and programmed cell death in the developing limb. Development $126,5515-22$

18. Hogan BL. (1996) Bone morphogenetic proteins: multifunctional regulators of vertebrate development. Genes Dev10,1580-94.

19. Winnier G, Blessing M, Labosky PA, Hogan BL. (1995) Bone morphogenetic protein-4 is required for mesoderm formation and patterning in the mouse. Genes Dev 9,2105-16.

20. Zhang H, Bradley A. (1996) Mice deficient for BMP2 are nonviable and have defects in amnion/chorion and cardiac development. Development 122,2977-86 
21. Miyazaki Y, Oshima K, Fogo A, Hogan BL, Ichikawa I. (2000) Bone morphogenetic protein 4 regulates the budding site and elongation of the mouse ureter. J Clin Invest 105,863-73.

22. Miyazaki Y, Oshima K, Fogo A, Ichikawa I. (2003) Evidence that bone morphogenetic protein 4 has multiple biological functions during kidney and urinary tract development. Kidney Int 63,835-44.

23. Dudley AT, Lyons KM, Robertson EJ. (1995) A requirement for bone morphogenetic protein-7 during development of the mammalian kidney and eye. Genes Dev 9,2795-807.

24. Luo G, Hofmann C, Bronckers AL, Sohocki M, Bradley A, Karsenty G. (1995) BMP-7 is an inducer of nephrogenesis, and is also required for eye development and skeletal patterning. Genes Dev 9,2808-20.

25. Khokha MK, Hsu D, Brunet LJ, Dionne MS, Harland RM. (2003) Gremlin is the BMP antagonist required for maintenance of Shh and Fgf signals during limb patterning. Nat Genet 34,303-7.

26. McMahon R, Murphy M, Clarkson M, et al. (2000) IHG-2, a mesangial cell gene induced by high glucose, is human gremlin. Regulation by extracellular glucose concentration, cyclic mechanical strain, and transforming growth factor-beta1. J Biol Chem 275,9901-4.

27. Zuniga A, Haramis AP, McMahon AP, Zeller R. (1999) Signal relay by BMP antagonism controls the SHH/FGF4 feedback loop in vertebrate limb buds. Nature 401,598-602.

28. Michos O, Panman L, Vintersten K, Beier K, Zeller R, Zuniga A. (2004) Gremlin-mediated BMP antagonism induces the epithelial-mesenchymal feedback signaling controlling metanephric kidney and limb organogenesis. Development 131,3401-10.

29. Lee TI, Jenner RG, Boyer LA, et al. (2006) Control of developmental regulators by Polycomb in human embryonic stem cells. Cell 125,301-13.

30. Widschwendter M, Fiegl H, Egle D, et al. (2007) Epigenetic stem cell signature in cancer. Nat Genet 39,157-8.

31. Yamashita S, Tsujino Y, Moriguchi K, Tatematsu M, Ushijima T. (2006) Chemical genomic screening for methylation-silenced genes in gastric cancer cell lines using 5-aza-2'-deoxycytidine treatment and oligonucleotide microarray. Cancer Sci 97,64-71.

32. Suzuki M, Shigematsu H, Shames DS, et al. (2005) DNA methylation-associated inactivation of TGFbetarelated genes DRM/Gremlin, RUNX3, and HPP1 in human cancers. Br J Cancer 93,1029-37.

33. van den Brandt PA, Goldbohm RA, van 't Veer P, Volovics A, Hermus RJ, Sturmans F. (1990) A large-scale prospective cohort study on diet and cancer in The Netherlands. J Clin Epidemiol 43,285-95.

34. van Houwelingen KP, van Dijk BA, Hulsbergen-van de Kaa CA, et al.(2005) Prevalence of von HippelLindau gene mutations in sporadic renal cell carcinoma: results from The Netherlands cohort study. BMC Cancer 5,57.

35. Derks S, Lentjes MH, Hellebrekers DM, de Bruine AP, Herman JG, van Engeland M. (2004) Methylationspecific PCR unraveled. Cell Oncol 26,291-9.

36. Herman JG, Graff JR, Myohanen S, Nelkin BD, Baylin SB.(1996) Methylation-specific PCR: a novel PCR assay for methylation status of CpG islands. Proc Natl Acad Sci U S A 93,9821-6.

37. van Engeland M, Roemen GM, Brink M, et al. (2002) K-ras mutations and RASSF1A promoter methylation in colorectal cancer. Oncogene 21,3792-5.

38. Baldewijns MM, Thijssen VL, Van den Eynden GG, et al. (2007) High-grade clear cell renal cell carcinoma has a higher angiogenic activity than low-grade renal cell carcinoma based on histomorphological quantification and qRT-PCR mRNA expression profile. Br J Cancer 96,1888-95.

39. Van den Eynden GG, Van der Auwera I, Van Laere SJ, et al. (2005) Angiogenesis and hypoxia in lymph node metastases is predicted by the angiogenesis and hypoxia in the primary tumour in patients with breast cancer. Br J Cancer 93,1128-36.

40. Deng G, Peng E, Gum J, Terdiman J, Sleisenger M, Kim YS. (2002) Methylation of hMLH1 promoter correlates with the gene silencing with a region-specific manner in colorectal cancer. $\mathrm{Br} J$ Cancer 86,574-9. 
41. Baldewijns MM, van Vlodrop IJ, Schouten L, Soetekouw PM, de Bruine AP, van Engeland M. (2008) Genetics and epigenetics of renal cell cancer. Biochim Biophys Acta 1785,133-55.

42. Langenfeld EM, Langenfeld J. (2004) Bone morphogenetic protein-2 stimulates angiogenesis in developing tumors. Mol Cancer Res 2,141-9.

43. Rothhammer T, Bataille F, Spruss T, Eissner G, Bosserhoff AK. (2007) Functional implication of BMP4 expression on angiogenesis in malignant melanoma. Oncogene 26,4158-70.

44. Raida M, Clement JH, Leek RD, et al. (2005) Bone morphogenetic protein 2 (BMP-2) and induction of tumor angiogenesis. J Cancer Res Clin Oncol 131,741-50.

45. Maciel TT, Melo RS, Schor N, Campos AH. (2007) Gremlin promotes vascular smooth muscle cell proliferation and migration. J Mol Cell Cardiol.

46. Blish KR, Wang W, Willingham MC, et al. (2008) A Human Bone Morphogenetic Protein Antagonist Is Down-Regulated in Renal Cancer. Mol Biol Cell 19,457-464.

47. Chen B, Athanasiou M, Gu Q, Blair DG. (2002) Drm/Gremlin transcriptionally activates p21(Cip1) via a novel mechanism and inhibits neoplastic transformation. Biochem Biophys Res Commun 295,1135-41.

48. Alarmo EL, Rauta J, Kauraniemi P, Karhu R, Kuukasjarvi T, Kallioniemi A. (2006) Bone morphogenetic protein 7 is widely overexpressed in primary breast cancer. Genes Chromosomes Cancer 45,411-9.

49. Hsu MY, Rovinsky S, Penmatcha S, Herlyn M, Muirhead D. (2005) Bone morphogenetic proteins in melanoma: angel or devil? Cancer Metastasis Rev 24,251-63.

50. Miyazaki H, Watabe T, Kitamura T, Miyazono K. (2004) BMP signals inhibit proliferation and in vivo tumor growth of androgen-insensitive prostate carcinoma cells. Oncogene 23,9326-35.

51. Aubin J, Davy A, Soriano P. (2004) In vivo convergence of BMP and MAPK signaling pathways: impact of differential Smad1 phosphorylation on development and homeostasis. Genes Dev 18,1482-94.

52. Franzen A, Heldin NE. (2001) BMP-7-induced cell cycle arrest of anaplastic thyroid carcinoma cells via p21(CIP1) and p27(KIP1). Biochem Biophys Res Commun 285,773-81.

53. Ghosh-Choudhury N, Woodruff K, Qi W, Celeste A, Abboud SL, Ghosh Choudhury G. (2000) Bone morphogenetic protein-2 blocks MDA MB 231 human breast cancer cell proliferation by inhibiting cyclin-dependent kinase-mediated retinoblastoma protein phosphorylation. Biochem Biophys Res Commun 272,705-11.

54. Haudenschild DR, Palmer SM, Moseley TA, You Z, Reddi AH. (2004) Bone morphogenetic protein (BMP)6 signaling and BMP antagonist noggin in prostate cancer. Cancer Res 64,8276-84.

55. He XC, Zhang J, Tong WG, et al. (2004) BMP signaling inhibits intestinal stem cell self-renewal through suppression of Wnt-beta-catenin signaling. Nat Genet 36,1117-21.

56. Moustakas A, Heldin CH. (2005) Non-Smad TGF-beta signals. J Cell Sci 118,3573-84.

57. Pardali K, Kowanetz M, Heldin CH, Moustakas A. (2005) Smad pathway-specific transcriptional regulation of the cell cycle inhibitor p21(WAF1/Cip1). J Cell Physiol 204,260-72. 


\section{Chapter 9}

General discussion and conclusions 


\section{General discussion and conclusions}

Renal cell carcinoma (RCC), originating in the renal cortex, accounts for $2-3 \%$ of cancer incidence and results in over 100000 worldwide deaths annually. RCC is the most lethal urologic cancer and the sixth leading cause of cancer deaths in the developed nations. For those who present with metastasis, the overall clinical course of RCC varies; approximately $50 \%$ of patients survive $<1$ year and $10 \%$ survive for over 5 years[1]. For those who present with early stage disease, $30 \%$ of these patients will relapse after radical (partial) nephrectomy and will develop future metastasis. Standard chemotherapy has not been useful[2], and until recently, the only effective treatment for metastatic disease was cytokine-based immunotherapy with interferon$\alpha$ (IFN- $\alpha$ ) or interleukin-2 (IL-2), which have a response rate of approximately $15 \%$ [3]. Over the past few years, characterization of the VHL and HIF pathways has led to a more detailed understanding of RCC biology, with subsequent development of effective anti-angiogenic targeted therapy. Many $\mathrm{VHL} /$ hypoxia-induced regulated proteins are receptor tyrosine kinases (RTKs) and their ligands, involved in tumour driven angiogenesis, and small molecule RTK inhibitors (such as sunitinib, sorafenib and bevacizumab) show promising results in the treatment of metastatic RCC (chapter 3 and 7). The high vascularization, characteristic for $\mathrm{CCRCC}$, already suggests that angiogenesis is integral to its pathogenesis and explains why ccRCC is an attractive disease for angiogenesis research and application of anti-angiogenic targeted therapy.

Therefore, the first interest of this thesis was to assess angiogenesis parameters in cCRCC and to investigate whether these variables can serve as a measure or predictor of anti-angiogenesis therapy response and to examine whether differences in angiogenic activity can explain variable biological behaviour among ccRCCs.

The first experimental study (chapter 4) assesses the angiogenic activity of low-grade and high-grade ccRCC by means of morphological quantification (vessel density, endothelial cell proliferation) and qRT-PCR mRNA expression profile of angiogenic activators and inhibitors. This study showed higher active angiogenesis, reflected by a higher endothelial cell proliferation fraction (ECP\%), in high-grade when compared to lowgrade ccRCC. In concordance, ECP\% strongly correlated with a higher tumour cell proliferation rate (TCP\%). This strong association implies that ECP\% and TCP\% in CCRCCS may be driven by similar mechanisms. Alternatively, the growth rate of tumour cells may determine the angiogenic potential of the endothelial cells (EC) because our study demonstrated enhanced protein VEGF expression in high-grade ccRCC.

In contrast, microvessel density (MVD) appeared to be significantly lower in high-grade cCRCC versus low-grade ccRCC. This finding indicates that MVD is not a good parameter for tumour growth rate or for active angiogenesis in ccRCC. MVD is only a measure of the intercapillary distance in a certain microscopic field, but not a reflection of the angiogenic activity of a tumour[4]. This means that MVD is not a valid indicator to evaluate therapeutic angiostatic response in ccRCC. It is interesting that high-grade ccRCCs can exist with low MVD. Köhler et al[5] pointed out that MVD in normal renal 
cortex and medulla is already high and only slightly increased in Fuhrman grade 1 ccRCC. The decrease of MVD with increasing grade probably reflects the inability of the neovascularization to keep up with tumour cell proliferation. In spite of the low MVD, the vascular bed seems to give an adequate supply to the tumour tissue, as the maximum tumour diameter increases with grade. Though the amount of total tumour vascularization must increase rapidly in fast-growing tumours to support a rapidly increasing tumour mass, the density of the vessels does not need to be high. This is because of the fact that MVD is influenced by angiogenic as well as by non-angiogenic factors, such as the oxygen and nutrient consumption rates of the tumour cells. In human tumours the oxygen consumption rate is often substantially lower than that of the corresponding normal tissue[6]. In addition, tumour cells are known to tolerate oxygen deprivation and to be resistant to apoptosis under hypoxic conditions[7]. Furthermore, in previous experiments, the relative decrease of vascular dependence of cancer cells appears to be associated with features of increased malignancy [8,9]. Both the lowered oxygen consumption rate of tumour cells and their tolerance of hypoxic conditions permit increased intercapillary distance in tumours compared with their hypoxiasensitive counterparts and normal tissue. Alternatively, in high-grade ccRCC larger vessels are observed[10] with probable increased permeability of the vessel wall and altered fluid dynamic properties, both compensating the reduced vascular bed[11,12]. Another interesting finding of our first study was that high-grade ccRCCs were characterised by a significantly lower HIF-1 $\alpha$ expression than low-grade ccRCC. This may be due to a progressive switch to HIF- $2 \alpha$ response during tumour progression. Earlier reports indicated that during renal tumourigenesis, HIF-2 $\alpha$ becomes progressively more evident than HIF-1 $\alpha[13,14]$. In ccRCC it is suggested that HIF-2 $\alpha$ has more oncogenic properties than HIF-1 $\alpha$, encoding protumorigenic genes such as cyclin D1, transforming growth factor- $\alpha$ and VEGF $[13,15]$. Consequently, HIF-2 $\alpha$ driven expression of VEGF, a potent mediator of EC activation, can explain the high ECP\% in high-grade cCRCC.

Furthermore, we demonstrated that ccRCC with high MVD and low ECP\%, largely representing low-grade $C C R C C$, revealed significantly higher expression of placental growth factor, plateled-derived growth factor and angiopoietin-1, which are related to vessel maturation[16-19]. This finding suggests the presence of more immature vessels in high-grade ccRCC. Poorly differentiated vessels may provide easier access for cancer cells to the circulation, explaining the high metastatic potential of high-grade ccRCC. Yao et al demonstrated that an increased number of undifferentiated microvessels in cCRCC significantly correlated with higher tumour grades and multivariate analyses even showed that undifferentiated MVD was an independent prognostic factor[20]. In contrast with microvessel density, the state of vascular differentiation may be a valid indicator for therapeutic response. Previously, the identification of angiopoietins[21] and the phenotype of plateled-derived growth factor- $\beta$-deficient mice (inability to recruit pericytes)[17] has focused attention on the molecular mechanisms of blood vessel maturation mediated by recruitment of pericytes. The mature phenotype of the 
quiescent organ vasculature in most normal organs is characterized by an extensive coverage with pericytes that appear to play a role in controlling the quiescent endothelial cell phenotype. The concept that tumour vessels can be selectively targeted without affecting the quiescent organ vasculature is based on the fact that the molecular phenotype of immature, angiogenic intratumoral blood vessels (with less or absence of pericytes) is distinctly different from that of resting blood vessels. Numerous animal studies have shown that the activated, angiogenic intratumoral neovasculature can be selectively targeted without affecting the normal organ vasculature[22]. Antiangiogenic therapies are thought to induce the pruning of immature and inefficient vessels and to promote the maturation of the remaining vessels[23]. As immature vasculature is believed to be more vulnerable for therapeutic targeting[24], a better response to antiangiogenic drugs might be expected in high-grade ccRCC. However, antiangiogenic therapy can cause resistance to therapy response, by selection and overgrowth of tumour-cell variants that are hypoxia resistant and thus less dependent on angiogenesis[25]. Considering the hypothesis that high-grade ccRCC become less vascular dependent, response to antiangiogenic drugs may yet be disappointing in this RCC subgroup. On the other hand, anti-VEGF treatment probably has direct effects on the tumour cells of RCC, since activated (phosphorylated) VEGFR-2 has been shown to be expressed on RCC cells, independent of grade[26]. Low-grade ccRCC with low ongoing angiogenic activity may not benefit much from antiangiogenic therapies that depend on the rate of EC proliferation.

This stresses the importance of assessing the composition of the tumour vessels (e.g., the angiogenic status (ECP\%) and maturity (such as presence of pericytes) in clinical practice to identify patients that will benefit most from antiangiogenic therapy.

The second interest of this thesis was to investigate whether differences in angiogenic activity and biological behaviour can be explained by different alterations in the $\mathrm{VHL}$ gene, a common event in ccRCC tumorigenesis and key player in HIF driven angiogenesis (chapter 5). For this, VHL mutation analysis (by means of direct sequencing) and VHL promoter CpG island methylation analysis (by means of methylation-specific PCR) were performed. No significant correlations were found between $V H L$ mutation (presence/type) or $V H L$ promoter hypermethylation and tumour parameters (grade, stage, TCP\%) or angiogenesis related variables (MVD, ECP\%,VEGF, HIF-1 $\alpha$ ). Regulation of angiogenesis and tumour progression of cCRCC is apparently not directly influenced by $V H L$ alterations. The equal frequencies of $V H L$ alterations between tumour stages 1,2 and 3 support the hypothesis that $V H L$ inactivation is an early event in CcRCC tumorigenesis. However, subsequent to this inactivation, further genetic and probably also epigenetic events in the HIF pathway, which plays a central role in renal angiogenesis and carcinogenesis, are needed for tumour progression. The HIF pathway is complex and not only regulated by constitutive HIF signalling through defective $V H L$ in case of ccRCC or by hypoxia. Oncogenic HIF signalling (PI3K/AKT pathway[27,28]/MAPK pathway[29,30]), as well as modulations in HIF downstream pathways (including a complex 
of paracrine and autocrine effects of angiogenic growth factors) can modify tumourdriven angiogenesis.

Another potential insight from patients with ccRCC with different $V H L$ mutations is that there are some mutations which disturb HIF regulation but appear to be associated with no excess risk of ccRCC, or reduced risk[31]. One explanation for this may be that the effect of these mutations do not completely abolish the ability of $\mathrm{pVHL}$ to regulate HIF. Alternatively, it may be that some other aspect of VHL function is preserved when these mutations occur, and that HIF dysregulation alone is not sufficient to predispose to $\mathrm{CCRCC}$.

As stated above, $V H L$ inactivation is common in sporadic ccRCC (in about $70-80 \%$ of sporadic ccRCC, including 50\%-75\% VHL mutations[32-34] and 5-20\% VHL promoter hypermethylation[35-38]). An interesting question is whether the remaining $20 \%$ of cCRCC ( $27 \%$ in our study) have a VHL-HIF pathway which functions normally. It appears likely that they do, since this subset of tumours show patchy HIF activation, especially in the hypoxic regions around areas of necrosis, in contrast with the more diffuse HIF expression in the $V H L$ defective $\operatorname{ccRCC}[39,40]$. Furthermore some $\mathrm{ccRCC}$ derived cell lines regulate HIF normally, for example, Caki-1[40]. The absence of somatic VHL alterations in some ccRCCs also implies the involvement of other genetic events in renal carcinogenesis. Additional inactivation of tumour suppressor genes located at chromosome 3p12-p21 appears to be necessary in the development of ccRCC, irrespective of $V H L$ inactivation[41,42]. Also other regions of genetic loss on chromosomes other than the frequently involved chromosome 3p, have been identified in ccRCC[43-45]. Recently, an array-based comparative genomic hybridization analysis indicated that in addition to loss of chromosome $3 p$, gain of chromosomes $5 q$ and 7 may be indispensable for the development of ccRCC in both identified clusters. Additional loss of chromosomes $1 p, 4,9,13 q$ and $14 q$ may promote the genetic pathways to more malignant ccRCCs[46].

Since hemangiogenesis is a key feature in renal carcinogenesis, the interest of our third experimental study (chapter 6) was to investigate whether there is also lymphangiogenesis present in ccRCC. This study was performed by means of a Ki67-D2-40 double immunohistochemical staining (lymph vessel density and lymph endothelial proliferation fraction (LECP\%)) and qRT-PCR mRNA expression profile of lymphangiogenic factors.

Because ccRCC appear to be angiogenic tumours and angiogenic growth factors exhibit overlapping lymphangiogenic and hemangiogenic activity[47], lymphangiogenesis might be expected in ccRCC. Recent studies have revealed that tumours can actively induce the formation of lymphatic vessels, and that tumour lymphangiogenesis is correlated with lymph node metastasis in experimental cancer models and in several types of human cancers[48]. Studies in animal tumour models showed that increased levels of VEGF-C and/or VEGF-D promote active tumour lymphangiogenesis and lymphatic tumour spread to regional lymph nodes, and that these effects can be sup- 
pressed by blocking VEGFR-3 signalling[49-52]. VEGF-A can also act as a tumour lymphangiogenesis factor[53].

In cCRCC, however, only limited ongoing intratumoral lymphangiogenesis (LECP\%) was observed. As the extent of tumour-associated lymphangiogenesis can serve as a potent predictor of lymph node metastasis[54], our finding may explain the low prevalence of lymph node metastasis in ccRCC. Furthermore, this finding, together with the result of our first experimental study, indicates that during renal tumourigenesis, lymphangiogenesis is of less consequence and hemangiogenesis predominates. This observation may provide a clue to better understand why ccRCC prefer hematogenous dissemination to lymphatic spread.

Since no evident lymphangiogenesis can be demonstrated in ccRCC, the lymph vessels located in the center and at the margin of the tumor are probably preexisting lymph vessels.

The lack of a relationship between the number of lymph vessels and lymph node metastases can be explained by the small number of intratumoral lymphatic vessels and by the fact that intratumoral lymphatic vessels may not be completely functional. At least in animal models, these vessels collapse under high intratumoral pressure and in at least one study they were not conductive of lymphatic metastasis[55,56]. Furthermore peritumoral lymphatics within the pseudocapsule or invasive edge of the investigated ccRCC were irregular and often collapsed and might not be able to serve lymphatic spread. This hypothesis is supported by an earlier study of Bonsib et al. who observed intralymphatic tumour emboli in 7 of 40 ccRCCs. In 4 of these 7 cases, intralymphatic tumour emboli were detected in lymph vessels outside the tumour (mostly renal hilus), and their presence correlated with lymph node involvement. However, intralymphatic tumour emboli, localized in peritumoral lymph vessels, were not associated with lymph node involvement[57,58].

As stated above, angiogenic capacity in CCRCC is not directly influenced by VHL alterations and this suggests that additional HIF upstream and downstream events interfere with tumour driven angiogenesis. The past few years there is an explosion of interest in the epigenetic regulation of gene expression in cancer. Epigenetic silencing of tumour suppressor genes by promoter DNA hypermethylation and/or histone modifications plays an important role in the pathogenesis of sporadic and/or hereditary forms of RCC, and are correlated with tumour aggressiveness and poorer patient outcome (chapter 7). Moreover, regional DNA hypermethylation in precancerous conditions appears to generate more malignant RCCs[59] and a significant association is demonstrated between regional DNA hypermethylation and genetic clustering of ccRCC, based on copy number alterations[46].

Since the role of epigenetic alteration of gene expression is becoming more and more acknowledged in renal carcinogenesis, we were interested in epigenetically silenced tumour suppressor genes, which were also involved in angiogenesis. To that end, our fourth experimental study (chapter 8) was focused on Gremlin 1 (GREM1), a bone morphogenic protein (BMP) antagonist and member of the transforming growth fac- 
tor- $\beta$ (TGF- $\beta$ ) family, described to be silenced via promoter CpG island hypermethylation in several other human malignancies[60,61]. BMP-independent tumour suppressor (-like) functions of GREM1 are demonstrated in other tumour cell lines[62] and a potential role of GREM1 in tumour-driven angiogenesis has been postulated[63]. No data of GREM1 promoter CpG island methylation were reported before, so we evaluated the presence of GREM1 promoter hypermethylation in CCRCC cellines and samples of patients with primary cCRCCs, and investigated whether GREM1 silencing is related to variable tumour behaviour and angiogenesis. A strong positive correlation was observed between GREM1 promoter hypermethylation and active angiogenesis (ECP) as well as tumour behaviour (tumour stage, Fuhrman grade and overall survival), indicating a putative role for GREM1 in CCRCC progression and tumour-driven angiogenesis. This was also confirmed by a second independent population based series, in which GREM1 appeared to be an independent prognostic factor.

The exact mechanism on how GREM1 acts as a putative tumour suppressor gene and modulating tumour-driven angiogenesis in cCRCC needs to be unravelled in future assays. Furthermore, in general, current understanding of epigenetic alterations occurring during renal carcinogenesis is far from complete and has to be elucidated in further research, which will contribute to development of targeted epigenetic therapy. In vitro and preclinical studies showed that the clinical application of reversing epigenetic aberrations in tumour cells, called epigenetic therapy, is an exciting strategy for cancer treatment[64]. Many agents have been discovered that inhibit DNA methylation (DNMT inhibitors) or histone deacetylation (HDAC inhibitors), and the value of these compounds will be established by ongoing clinical trials[65]. As some of the tumour suppressor genes, which are epigenetically silenced in tumour cells, may exhibit angiogenesis inhibiting properties, indirect angiostatic effects of demethylating agents and HDAC inhibitors might be expected[65]. Some reports related the angiostatic activity of HDAC inhibitors to repression of HIF-1 $\alpha$ and/or VEGF in tumour cells[66-69]. Previous studies also showed that HDAC inhibitors are potent angiostatic agents, directly inhibiting endothelial cell growth and angiogenesis in vitro and in vivo[65,66,70]. However, in contrast with the extensively described effects of DNMT and HDAC inhibitors on gene expression in tumour cells, there is more to learn about the direct effects of these drugs on $\mathrm{EC}$ gene expression, as well as on the role of epigenetic mechanisms in regulating transcription of EC genes during tumour angiogenesis.

In summary, the results of this thesis suggest heterogeneity in angiogenic activity and vessel maturation of CCRCC, which to a large extent is linked to nuclear grade. These differences in angiogenesis biology might have impact on the effects of antiangiogenic treatment of ccRCC. Because of the limitations of MVD as a surrogate end point, supplementary assays for evaluating the efficacy of antiangiogenic agents are needed. To that end, assays that reflect the angiogenic status (ECP\%) or quantify the maturation state of tumour blood vessels (such as presence of pericytes, concentration ratio of angiopoietins (Ang-1 and Ang-2)[21]), may be used in clinical practice. In contrast with 
hemangiogenesis, limited intratumoral lymphangiogenesis occurs in ccRCC. This may explain the low prevalence of lymph node metastasis in cCRCC and common hematogenous metastatic route.

The absence of a direct association between VHL alterations and angiogenesis/tumour variables indicates that additional genetic/epigenetic events should be considered to explain angiogenic heterogeneity and diverse tumour behaviour. To that end, epigenetic changes were explored in CCRCC and GREM1 promoter hypermethylation was demonstrated to be associated with angiogenic activity and variable tumour behaviour.

Current understanding of the genetic and epigenetic alterations occurring during renal carcinogenesis and their relation with tumour driven angiogenesis, however, is far from complete.

Each step in the angiogenic regulatory pathway represents a potential target for therapy and antiangiogenesis has become an important component of cancer treatment in RCC. However, despite the positive results with angiogenesis inhibitors, clinical resistance remains an issue as these compounds block only one proangiogenic protein (such as for instance VEGF), whereas during renal tumourigenesis multiple proangiogenic proteins are produced, which allow the tumour to evade angiogenic blockade. Therefore, additional targets and pathways need to be exploited to broaden the antiangiogenic spectrum. Unravelling of the global network of multi-factorial stimulatory and inhibitory circuits regulating the angiogenic switch will serve the means to sustainably suppress angiogenesis, using pharmacological agents that block both primary and secondary/alternative proangiogenic signalling pathways, and/or hyperactivate endogenous antiangiogenic signals.

With antiangiogenic therapy, the challenge comes in providing the proper guidelines for using combination therapies (such as several antiangiogenic agents and epigenetic targeted therapy, in combination or combined with immunotherapy), optimisation of treatment schedules, and accurately assessing patient response (development of appropriate surrogate markers[71]).

Ultimately, the evolution of RCC is probably not entirely dependent on HIF and RTKs, and also other pathways, that drive ccRCC progression, need to be discovered with novel inhibitors to be synthesised and tested clinically. 


\section{References}

1. Kovacs G, Akhtar M, Beckwith BJ, et al. The Heidelberg classification of renal cell tumours. J Pathol 1997;183:131-3.

2. Gelb AB. Renal cell carcinoma: current prognostic factors. Union Internationale Contre le Cancer (UICC) and the American Joint Committee on Cancer (AJCC). Cancer 1997;80:981-6.

3. Kaelin WG, Jr. The von Hippel-Lindau tumor suppressor gene and kidney cancer. Clin Cancer Res 2004;10:6290S-5S.

4. van Dijk BA, Schouten LJ, Oosterwijk E, et al. Cigarette smoking, von Hippel-Lindau gene mutations and sporadic renal cell carcinoma. Br J Cancer 2006;95:374-7.

5. Gnarra JR, Duan DR, Weng Y, et al. Molecular cloning of the von Hippel-Lindau tumor suppressor gene and its role in renal carcinoma. Biochim Biophys Acta 1996;1242:201-10.

6. Richards FM. Molecular pathology of von HippelLindau disease and the VHL tumour suppressor gene. Expert Rev Mol Med 2001;2001:1-27.

7. Banks RE, Tirukonda P, Taylor C, et al. Genetic and epigenetic analysis of von Hippel-Lindau (VHL) gene alterations and relationship with clinical variables in sporadic renal cancer. Cancer Res 2006;66:200011.

8. Dulaimi E, Ibanez de Caceres I, Uzzo RG, et al. Promoter hypermethylation profile of kidney cancer. Clin Cancer Res 2004;10:3972-9.

9. Herman JG, Latif F, Weng $Y$, et al. Silencing of the VHL tumor-suppressor gene by DNA methylation in renal carcinoma. Proc Natl Acad Sci U S A 1994;91:9700-4.

10. Schraml P, Struckmann K, Hatz F, et al. VHL mutations and their correlation with tumour cell proliferation, microvessel density, and patient prognosis in clear cell renal cell carcinoma. J Pathol 2002;196:186-93.

11. Smits KM, Schouten LJ, van Dijk BA, et al. Genetic and epigenetic alterations in the von hippel-lindau gene: the influence on renal cancer prognosis. Clin Cancer Res 2008;14:782-7.

12. Stabile H, Mitola S, Moroni E, et al. Bone morphogenic protein antagonist Drm/gremlin is a novel proangiogenic factor. Blood 2007;109:1834-40.

13. Hsu DR, Economides AN, Wang X, Eimon PM, Harland RM. The Xenopus dorsalizing factor Gremlin identifies a novel family of secreted proteins that antagonize BMP activities. Mol Cell 1998;1:673-83.

14. Topol LZ, Marx M, Laugier D, et al. Identification of drm, a novel gene whose expression is suppressed in transformed cells and which can inhibit growth of normal but not transformed cells in culture. Mol Cell Biol 1997;17:4801-10.

15. Topol LZ, Modi WS, Koochekpour S, Blair DG. DRM/GREMLIN (CKTSF1B1) maps to human chromosome 15 and is highly expressed in adult and fetal brain. Cytogenet Cell Genet 2000;89:79-84.

16. Topol LZ, Bardot B, Zhang Q, et al. Biosynthesis, post-translation modification, and functional characterization of Drm/Gremlin. J Biol Chem 2000;275:8785-93.

17. Merino R, Rodriguez-Leon J, Macias D, Ganan Y, Economides AN, Hurle JM. The BMP antagonist Gremlin regulates outgrowth, chondrogenesis and programmed cell death in the developing limb. Development 1999;126:5515-22.

18. Hogan BL. Bone morphogenetic proteins: multifunctional regulators of vertebrate development. Genes Dev 1996;10:1580-94.

19. Winnier G, Blessing M, Labosky PA, Hogan BL. Bone morphogenetic protein-4 is required for mesoderm formation and patterning in the mouse. Genes Dev 1995;9:2105-16.

20. Zhang $\mathrm{H}$, Bradley A. Mice deficient for BMP2 are nonviable and have defects in amnion/chorion and cardiac development. Development 1996;122:2977-86. 
21. Miyazaki Y, Oshima K, Fogo A, Hogan BL, Ichikawa I. Bone morphogenetic protein 4 regulates the budding site and elongation of the mouse ureter. J Clin Invest 2000;105:863-73.

22. Miyazaki Y, Oshima K, Fogo A, Ichikawa I. Evidence that bone morphogenetic protein 4 has multiple biological functions during kidney and urinary tract development. Kidney Int 2003;63:835-44.

23. Dudley AT, Lyons KM, Robertson EJ. A requirement for bone morphogenetic protein-7 during development of the mammalian kidney and eye. Genes Dev 1995;9:2795-807.

24. Luo G, Hofmann C, Bronckers AL, Sohocki M, Bradley A, Karsenty G. BMP-7 is an inducer of nephrogenesis, and is also required for eye development and skeletal patterning. Genes Dev 1995;9:2808-20.

25. Khokha MK, Hsu D, Brunet LJ, Dionne MS, Harland RM. Gremlin is the BMP antagonist required for maintenance of Shh and Fgf signals during limb patterning. Nat Genet 2003;34:303-7.

26. McMahon R, Murphy M, Clarkson M, et al. IHG-2, a mesangial cell gene induced by high glucose, is human gremlin. Regulation by extracellular glucose concentration, cyclic mechanical strain, and transforming growth factor-beta1. J Biol Chem 2000;275:9901-4.

27. Zuniga A, Haramis AP, McMahon AP, Zeller R. Signal relay by BMP antagonism controls the SHH/FGF4 feedback loop in vertebrate limb buds. Nature 1999;401:598-602.

28. Michos O, Panman L, Vintersten K, Beier K, Zeller R, Zuniga A. Gremlin-mediated BMP antagonism induces the epithelial-mesenchymal feedback signaling controlling metanephric kidney and limb organogenesis. Development 2004;131:3401-10.

29. Lee TI, Jenner RG, Boyer LA, et al. Control of developmental regulators by Polycomb in human embryonic stem cells. Cell 2006;125:301-13.

30. Widschwendter M, Fiegl H, Egle D, et al. Epigenetic stem cell signature in cancer. Nat Genet 2007;39:157-8.

31. Yamashita S, Tsujino Y, Moriguchi K, Tatematsu M, Ushijima T. Chemical genomic screening for methylation-silenced genes in gastric cancer cell lines using 5-aza-2'-deoxycytidine treatment and oligonucleotide microarray. Cancer Sci 2006;97:64-71.

32. Suzuki M, Shigematsu $H$, Shames DS, et al. DNA methylation-associated inactivation of TGFbeta-related genes DRM/Gremlin, RUNX3, and HPP1 in human cancers. Br J Cancer 2005;93:1029-37.

33. van den Brandt PA, Goldbohm RA, van 't Veer P, Volovics A, Hermus RJ, Sturmans F. A large-scale prospective cohort study on diet and cancer in The Netherlands. J Clin Epidemiol 1990;43:285-95.

34. van Houwelingen KP, van Dijk BA, Hulsbergen-van de Kaa CA, et al. Prevalence of von Hippel-Lindau gene mutations in sporadic renal cell carcinoma: results from The Netherlands cohort study. BMC Cancer 2005;5:57

35. Derks S, Lentjes MH, Hellebrekers DM, de Bruine AP, Herman JG, van Engeland M. Methylation-specific PCR unraveled. Cell Oncol 2004;26:291-9.

36. Herman JG, Graff JR, Myohanen S, Nelkin BD, Baylin SB. Methylation-specific PCR: a novel PCR assay for methylation status of CpG islands. Proc Natl Acad Sci U S A 1996;93:9821-6.

37. van Engeland $M$, Roemen GM, Brink M, et al. K-ras mutations and RASSF1A promoter methylation in colorectal cancer. Oncogene 2002;21:3792-5.

38. Baldewijns MM, Thijssen VL, Van den Eynden GG, et al. High-grade clear cell renal cell carcinoma has a higher angiogenic activity than low-grade renal cell carcinoma based on histomorphological quantification and qRT-PCR mRNA expression profile. Br J Cancer 2007;96:1888-95

39. Van den Eynden GG, Van der Auwera I, Van Laere SJ, et al. Angiogenesis and hypoxia in lymph node metastases is predicted by the angiogenesis and hypoxia in the primary tumour in patients with breast cancer. Br J Cancer 2005;93:1128-36

40. Deng G, Peng E, Gum J, Terdiman J, Sleisenger M, Kim YS. Methylation of hMLH1 promoter correlates with the gene silencing with a region-specific manner in colorectal cancer. Br J Cancer 2002;86:574-9. 
41. Baldewijns MM, van Vlodrop IJ, Schouten $\sqcup$, Soetekouw PM, de Bruine AP, van Engeland M. Genetics and epigenetics of renal cell cancer. Biochim Biophys Acta 2008;1785:133-55.

42. Langenfeld EM, Langenfeld J. Bone morphogenetic protein-2 stimulates angiogenesis in developing tumors. Mol Cancer Res 2004;2:141-9.

43. Rothhammer T, Bataille F, Spruss T, Eissner G, Bosserhoff AK. Functional implication of BMP4 expression on angiogenesis in malignant melanoma. Oncogene 2007;26:4158-70.

44. Raida M, Clement JH, Leek RD, et al. Bone morphogenetic protein 2 (BMP-2) and induction of tumor angiogenesis. J Cancer Res Clin Oncol 2005;131:741-50.

45. Maciel TT, Melo RS, Schor N, Campos AH. Gremlin promotes vascular smooth muscle cell proliferation and migration. J Mol Cell Cardiol 2007.

46. Blish KR, Wang W, Willingham MC, et al. A Human Bone Morphogenetic Protein Antagonist Is DownRegulated in Renal Cancer. Mol Biol Cell 2008;19:457-464.

47. Chen B, Athanasiou M, Gu Q, Blair DG. Drm/Gremlin transcriptionally activates p21(Cip1) via a novel mechanism and inhibits neoplastic transformation. Biochem Biophys Res Commun 2002;295:1135-41.

48. Alarmo EL, Rauta J, Kauraniemi P, Karhu R, Kuukasjarvi T, Kallioniemi A. Bone morphogenetic protein 7 is widely overexpressed in primary breast cancer. Genes Chromosomes Cancer 2006;45:411-9.

49. Hsu MY, Rovinsky S, Penmatcha S, Herlyn M, Muirhead D. Bone morphogenetic proteins in melanoma: angel or devil? Cancer Metastasis Rev 2005;24:251-63.

50. Miyazaki $H$, Watabe $T$, Kitamura T, Miyazono K. BMP signals inhibit proliferation and in vivo tumor growth of androgen-insensitive prostate carcinoma cells. Oncogene 2004;23:9326-35.

51. Aubin J, Davy A, Soriano P. In vivo convergence of BMP and MAPK signaling pathways: impact of differential Smad1 phosphorylation on development and homeostasis. Genes Dev 2004;18:1482-94.

52. Franzen A, Heldin NE. BMP-7-induced cell cycle arrest of anaplastic thyroid carcinoma cells via p21(CIP1) and p27(KIP1). Biochem Biophys Res Commun 2001;285:773-81.

53. Ghosh-Choudhury N, Woodruff K, Qi W, Celeste A, Abboud SL, Ghosh Choudhury G. Bone morphogenetic protein-2 blocks MDA MB 231 human breast cancer cell proliferation by inhibiting cyclindependent kinase-mediated retinoblastoma protein phosphorylation. Biochem Biophys Res Commun 2000;272:705-11.

54. Haudenschild DR, Palmer SM, Moseley TA, You Z, Reddi AH. Bone morphogenetic protein (BMP)- 6 signaling and BMP antagonist noggin in prostate cancer. Cancer Res 2004;64:8276-84.

55. He XC, Zhang J, Tong WG, et al. BMP signaling inhibits intestinal stem cell self-renewal through suppression of Wnt-beta-catenin signaling. Nat Genet 2004;36:1117-21.

56. Moustakas A, Heldin CH. Non-Smad TGF-beta signals. J Cell Sci 2005;118:3573-84.

57. Pardali K, Kowanetz $\mathrm{M}$, Heldin $\mathrm{CH}$, Moustakas A. Smad pathway-specific transcriptional regulation of the cell cycle inhibitor p21(WAF1/Cip1). J Cell Physiol 2005;204:260-72. 


\section{Summary}




\section{Summary}

Renal cell carcinoma (RCC) is a heterogenous disease, comprising different histological subtypes, each with a different underlying biology, different clinical course and different response to therapy. Most sporadic RCCs are clear cell renal cell carcinomas (ccRCC, 75\%) followed in frequency by papillary type I and II tumours (pRCC, 15\%) and chromophobe RCCs (chrRCC, 5\%).

RCCs are characterized by a lack of early symptoms, resulting in a quarter of patients presenting with advanced disease. It is the most lethal urological cancer and the sixth leading cause of cancer death in the developed nations. Surgery is the only timeproven therapeutic option in localized RCC. Standard chemotherapy has not been succesful, and until recently, the only effective treatment for metastatic disease was cytokine-based immunotherapy with interferon- $\alpha$ (IFN- $\alpha$ ) or interleukin-2 (IL-2), with a response rate of only $15 \%$ (chapter 2 ).

The high vascularization, which is characteristic for ccRCC, suggests that angiogenesis, the induction of a new vasculature, is integral to its pathogenesis. To that end, hypoxia-inducible factor (HIF) plays an important role in renal tumourigenesis by transcriptional activation of genes involved in angiogenesis, glucose metabolism and tumour cell proliferation (chapter 3). Over the past few years, characterization of the VHL and HIF pathways has led to a more detailed understanding of RCC biology, with subsequent development of effective anti-angiogenic targeted therapy. Many VHL/hypoxia-induced regulated proteins are receptor tyrosine kinases (RTKs) and their ligands, involved in tumour driven angiogenesis, and small molecule RTK inhibitors (such as sunitinib and sorafenib) show promising results in the treatment of metastatic RCC.

The first interest of this thesis was to assess angiogenesis parameters in ccRCC and to investigate whether differences in angiogenic activity can explain variable biological behaviour among ccRCCs. In our first experimental study, we demonstrated a higher angiogenic activity in high grade ccRCC, compared to low grade CCRCC, with heterogeneity in vessel maturation between both subgroups (chapter 4). Differences in angiogenesis might have impact on the effects of anti-angiogenic or anti-VEGF treatment in ccRCC.

The second interest of this thesis was to investigate whether differences in angiogenic activity and biological behaviour can be explained by specific alterations in the $V H L$ gene, a common event in ccRCC tumourigenesis and key player in HIF driven angiogenesis. In this study, no significant associations were found between $V H L$ alterations and tumour progression/angiogenesis suggesting that additional genetic and epigenetic events should be considered to explain diverse angiogenic and tumour proliferative behaviour (chapter 5 ).

Since hemangiogenesis is a key feature in renal carcinogenesis and since angiogenic growth factors exhibit overlapping lymphangiogenic and hemangiogenic activity, the aim of our third experimental study was to investigate the occurrence of lymphangio- 
genesis in ccRCC (chapter 6). The results of this study strongly suggest that limited intratumoural lymphangiogenesis occurs in CCRCC, which is not related to Fuhrman grade or tumour- and lymph node stage. Given the dual activity of several growth factors, stimulating both angiogenesis and lymphangiogenesis, this finding indirectly points to the fact that during renal tumourigenesis, hemangiogenesis predominates, probably explaining the common hematogenous spread of CCRCC and low prevalence of lymph node metastasis.

As stated above, the absence of significant associations between angiogenic parameters and $\mathrm{VHL}$ alterations in CCRCC, suggests that additional HIF upstream and downstream events interfere with tumour driven angiogenesis. In the last decades, knowledge on the role of epigenetics in the process of renal carcinogenesis has increased (chapter 7). Therefore, we were interested in epigenetic silencing of putative angiogenesis-modulating tumour suppressor genes. A genome-wide epigenetic screen identified Gremlin1(GREM1) as a gene frequently inactivated by promoter $\mathrm{CpG}$ island methylation in CCRCC. Therefore we investigated the influence of GREM1 promoter hypermethylation on angiogenesis/tumour behaviour and clinical outcome in cCRCC (chapter 8). This study showed a strong association between GREM1 promoter CpG island hypermethylation and increased angiogenesis and tumour progression, indicating a putative role for GREM1 in CCRCC carcinogenesis and tumour angiogenesis. However, the exact mechanism of GREM1 inactivation and its relation to tumour progression as well as its role in angiogenesis need to be further clarified.

In general, current understanding of epigenetic alterations occurring during renal carcinogenesis is far from complete and has to be elucidated in further research, which will contribute to development of targeted epigenetic therapy and may deliver new prognostic and early diagnostic markers. 


\section{Samenvatting}




\section{Samenvatting}

Na prostaat- en blaaskanker, is het niercelcarcinoom de meest voorkomende kwaadaardige urologische tumor bij de volwassene. Niercelcarcinomen ontstaan in de nierschors en vormen een heterogene groep van tumoren met een verschillende genetische achtergrond. Binnen de groep van de niercelcarcinomen, worden er meerdere histologische subtypes onderscheiden, waarbij de heldercellige niercelcarcinomen het meest frequent voorkomen.

Niercelcarcinomen worden, wegens het ontbreken van symptomen, vaak laattijdig gediagnosticeerd. Hierdoor is $25 \%$ van deze tumoren reeds gemetastaseerd bij diagnosestelling. Het gemetastaseerde niercelcarcinoom is resistent tegen chemo- en radiotherapie en er is slechts een geringe tumor respons (15\%) op immunotherapie (Interferon, Interleukine-2). Tot op heden, is operatieve verwijdering van de niertumor (tumor nefrectomie) bij een gunstige tumorstadiering de enige adequate curatieve therapie.

Het feit dat niercelcarcinomen zeer vaatrijke tumoren zijn, suggereert dat angiogenese een belangrijke rol speelt in de ontwikkeling van deze tumoren. Zowel preklinisch als klinisch onderzoek heeft aangetoond dat tumorgroei en metastasering afhankelijk zijn van angiogenese, een proces van vaatnieuwvorming vanuit voorafbestaande bloedvaten. Zodra een tumor groter wordt dan 2-3 $\mathrm{mm}^{3}$, ontstaat hypoxie (tekort aan zuurstof ) in het binnenste gedeelte van de tumor. De tumor kan dan uitsluitend verder groeien door de aanmaak van nieuwe bloedvaten. Hypoxie leidt tot activering van de transcriptiefactor 'hypoxie induceerbare factor' (HIF), die op zijn beurt een heel scala aan genen activeert, welke ondermeer tumorcelgroei, tumorcel overleving en angiogenese stimuleren. De belangrijkste angiogene groeifactor, die gereguleerd wordt door HIF, is de vasculaire endotheliale groeifactor (VEGF). Recente nieuwe inzichten in de pathogenese van niercelcarcinomen en meer bepaald van het heldercellige niercelcarcinoom, laten zien dat HIF en VEGF een sleutelrol spelen in de stimulatie van de tumor angiogenese. Bovendien treedt er bij de heldercellige niercelcarcinomen vaak een genetische fout op in het Von-Hippel-Lindau-tumorsuppressorgen. Tumor suppressorgenen zijn genen die de celgroei remmen en dus het ontstaan van een maligniteit helpen voorkomen. Uitschakeling van het $V H L$-gen zorgt ervoor dat HIF niet meer afgebroken kan worden en bijgevolg actief blijft. In dit geval wordt de angiogenese op eenzelfde manier gestimuleerd als bij hypoxie. Het feit dat niertumoren afhankelijk zijn van angiogenese, suggereert dat remming van angiogenese een veelbelovende manier van kankertherapie kan zijn bij deze groep van tumoren.

Anti-angiogenese onderzoek heeft geleid tot het ontwikkelen van een aantal nieuwe gerichte behandelingen, zoals blokkade van VEGF door middel van monoklonale antilichamen (bevacizumab) en remming van VEGF-receptortyrosinekinases (sunitinib, sorafenib). Ook het remmen van andere tumorale intracellulaire signaaltransductieroutes zoals de MAP-kinaseroute (met het oraal beschikbare sorafenib) of de AKT-mTOR route (met intraveneus temsirolimus) bieden nieuwe mogelijkheden voor de behandeling 
van patienten met gemetastaseerde niercelcarcinomen. In dit proefschrift geven hoofdstukken 2 en 3 een uitgebreider overzicht van de klinische en morfologische kenmerken, centrale rol van HIF en anti-angiogenese therapie bij niercelcarcinomen.

Het biologische gedrag van niercelcarcinomen blijft, ondanks de standaard stageringen gradering parameters, moeilijk voorspelbaar. Aangezien angiogenese een belangrijke rol speelt in de ontwikkeling van deze tumoren, werd in hoofdstuk 4 van dit proefschrift nagegaan of verschillen in angiogene activiteit het variabele gedrag van niertumoren kunnen verklaren. Hiervoor werden angiogenese parameters gemeten en vergeleken binnen een groep van 150 heldercellige niercelcarcinomen. Uit dit onderzoek blijkt dat hooggradige niercelcarcinomen een hogere angiogene activiteit tonen dan laaggradige tumoren, waarbij er ook aanwijzingen zijn voor verschillen in vaatmaturatie. Deze verschillen in angiogenese kunnen een rol spelen in de respons op antiangiogenese therapie.

Vervolgens werd in hoofdstuk 5 onderzocht of de verschillen in angiogene activiteit gerelateerd zijn aan defecten in het $\mathrm{VHL}$-tumorsuppressorgen. Bij deze studie werden er echter geen significante associaties gevonden tussen angiogene parameters en VHLinactivatie. Dit resultaat suggereert dat bijkomende genetische veranderingen nodig zijn voor de ontwikkeling en progressie van niertumoren.

Naast angiogenese, kan ook lymfangiogenese van belang zijn bij het metastaseringsproces van tumoren. Angiogene groeifactoren kunnen zowel angiogenese als lymfangiogenese (de vorming van nieuwe lymfevaten) stimuleren. Gezien de verhoogde expressie van angiogene groeifactoren binnen de groep van heldercellige niertumoren (hoofdstuk 4), zou in deze tumorgroep ook een verhoogde lymfangiogenese verwacht kunnen worden. Hoofdstuk 6 illustreert echter een beperkte lymfangiogenese, die niet geassocieerd is met tumorgraad, tumorstadium of lymfeklier metastasen. Deze bevinding vormt een mogelijke verklaring voor de lage incidentie van lymfeklier metastasen bij heldercellige niercelcarcinomen. Dit resultaat suggereert ook dat binnen de groep van de heldercellige niercelcarcinomen, de angiogene groeifactoren bij voorkeur de angiogenese stimuleren, wat ook zou kunnen verklaren waarom deze niertumoren frequenter via de bloedvaten metastaseren.

Het ontstaan van niercelcarcinomen is een complex proces, waarbij er naast het bekende en vaak uitgeschakelde $\mathrm{VHL}$-tumorsuppressorgen, ook andere fenomenen een rol spelen.

De laatste jaren verovert de epigenetica terrein in de zoektocht naar processen die verantwoordelijk zijn voor het ontstaan van kanker. Niet alleen veranderingen in de DNA sequentie zelf (genetica) kunnen leiden tot het ontstaan van kanker, maar ook veranderingen in de regulatie van genexpressie door modificaties van DNA transcriptie, de zogenaamde epigenetische processen, beïnvloeden in belangrijke mate de activiteit van genen. Waar de genetica refereert naar de overerving van informatie door middel van veranderingen in de nucleotidesequentie, refereert epigenetica naar overerfbare veranderingen in genfunctie die niet verklaard worden door veranderingen in nucleotidesequentie. Het Griekse woord "epi", wordt in het Nederlands vertaald als 
'op' of 'bij'. Epigenetica zou verwoord kunnen worden als 'dat wat bovenop of bij de genetica' komt. Epigenetische veranderingen kunnen gezien worden als een soort schakelaar die de activiteit van genen in een cel kan reguleren. Er zijn aanwijzingen dat een gezonde voeding een gunstig effect kan hebben op het optreden van epigenetische veranderingen. DNA-methylering geldt als een van de belangrijkste epigenetische processen en blijkt zelfs een even frequent voorkomende oorzaak te zijn van inactivatie van tumor suppressorgenen als mutaties (genetische veranderingen). Promoterhypermethylering van tumorsuppressorgenen zorgt voor inactivatie van genen die ondermeer de celgroei en angiogenese controleren. Ook bij niertumoren zijn er naast genetische defecten tal van epigenetische veranderingen beschreven, waarbij tumorsuppressorgenen uitgeschakeld worden, ten gevolge van promoterhypermethylering. Hoofdstuk 8 van dit proefschrift beschrijft de promoter methyleringsstatus van het Gremlin1(GREM1) gen, dat een belangrijke rol speelt in de embryonale nierontwikkeling. In de literatuur zijn er aanwijzen dat dit gen een tumorsuppressor functie zou kunnen vervullen en een rol zou kunnen spelen bij de tumorangiogenese. Reeds in andere tumoren werd aangetoond dat promoterhypermethylering van GREM1 kan optreden, maar bij de niercelcarcinomen was dit voorheen nog niet onderzocht. In onze studie wordt er bij de heldercellige niercelcarcinomen GREM1-promoterhypermethylering aangetoond, waarbij niertumoren met GREM1-promoterhypermethylering gekenmerkt worden door een toegenomen tumor angiogenese en tumorprogressie. Dit werd bevestigd in een tweede serie van heldercellige niercelcarcinomen, waarin GREM1-promoterhypermethylering zich zelfs als een onafhankelijke prognostische factor bleek te gedragen. De exacte functie van GREM1- uitschakeling bij de ontwikkeling van nierkanker dient echter nog nader onderzocht te worden. Ook zal er binnen de groep van de niercelcarcinomen nog verder onderzoek naar de mechanismen van andere door methylering uitgeschakelde potentiele tumorsuppressor genen moeten gebeuren. Dit onderzoek kan zowel gevolgen hebben voor (vroeg-) diagnostiek als nieuwe medicamenteuze therapievormen.

DNA methylering treedt namelijk in de vroege fase van de tumorontwikkeling op en is mogelijk reeds aanwezig voordat er macroscopische of microscopische tumorgroei zichtbaar is. Dit maakt methylering een interessante biomarker voor vroegtijdige screening van kwaadaardige tumoren. Tot slot is methylering een proces dat farmacologisch reversibel is. Hierdoor is methylering ook een interessant doelwit voor antikankerbehandeling. 
Full colour figures 


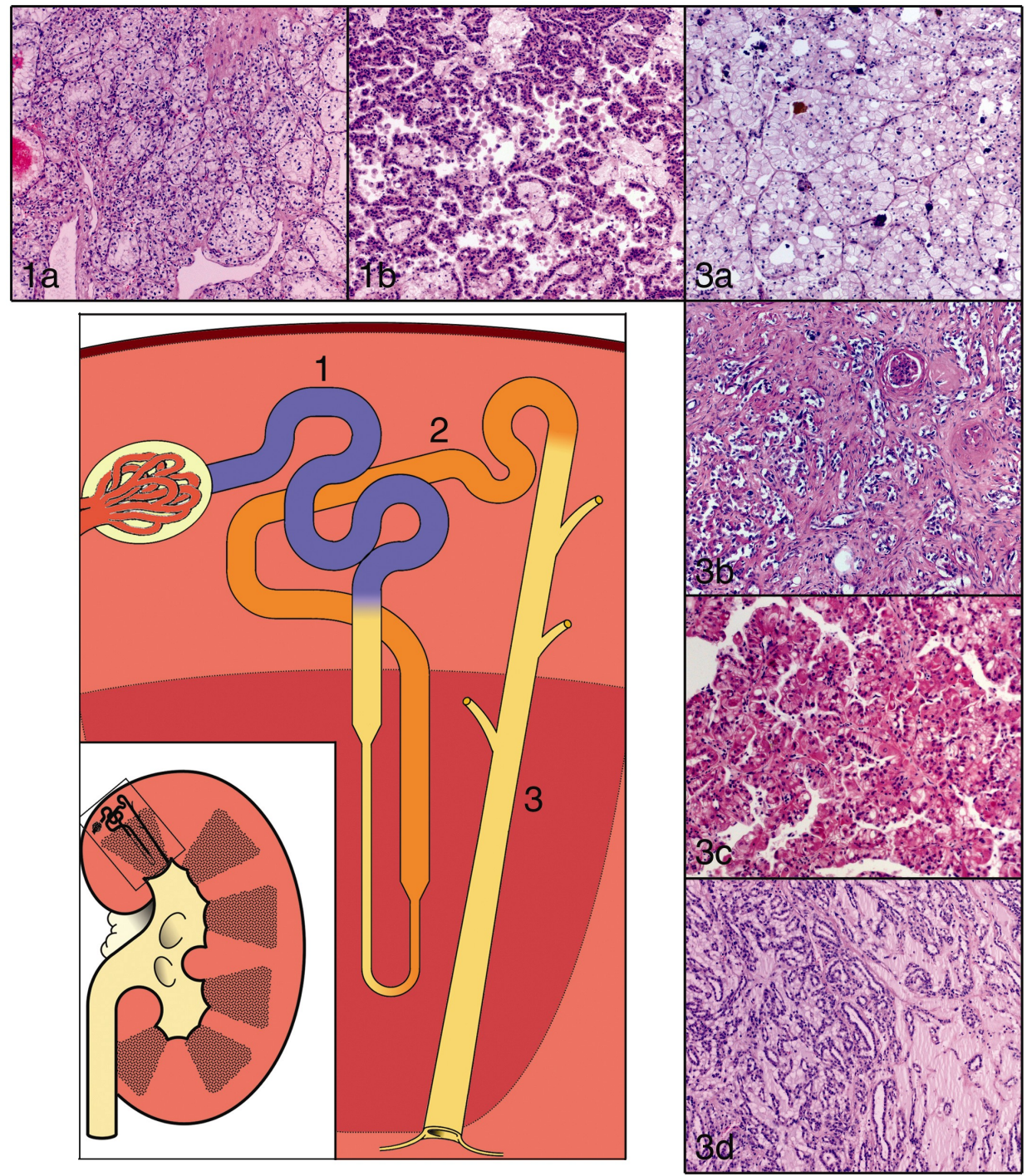

Figure 1: Classification of RCC subtypes: cell of origin

The nephron, consists of the renal corpuscle (1) and renal tubule, which is divided into four histophysiological zones: proximal convoluted tubule (2), loop of Henle (3), distal convoluted tubule (4) and collecting tubule (5). The different RCC subtypes are thought to originate from different parts of the renal tubular system: mainly the proximal convoluted tubule ( $2 \mathrm{a}$ clear cell RCC and $2 \mathrm{~b}$ papillary RCC) and the collecting tubule (5a chromophobe RCC, $5 \mathrm{~b}$ collecting duct carcinoma, and $5 \mathrm{c}$ mucinous tubular and spindle cell carcinoma). 


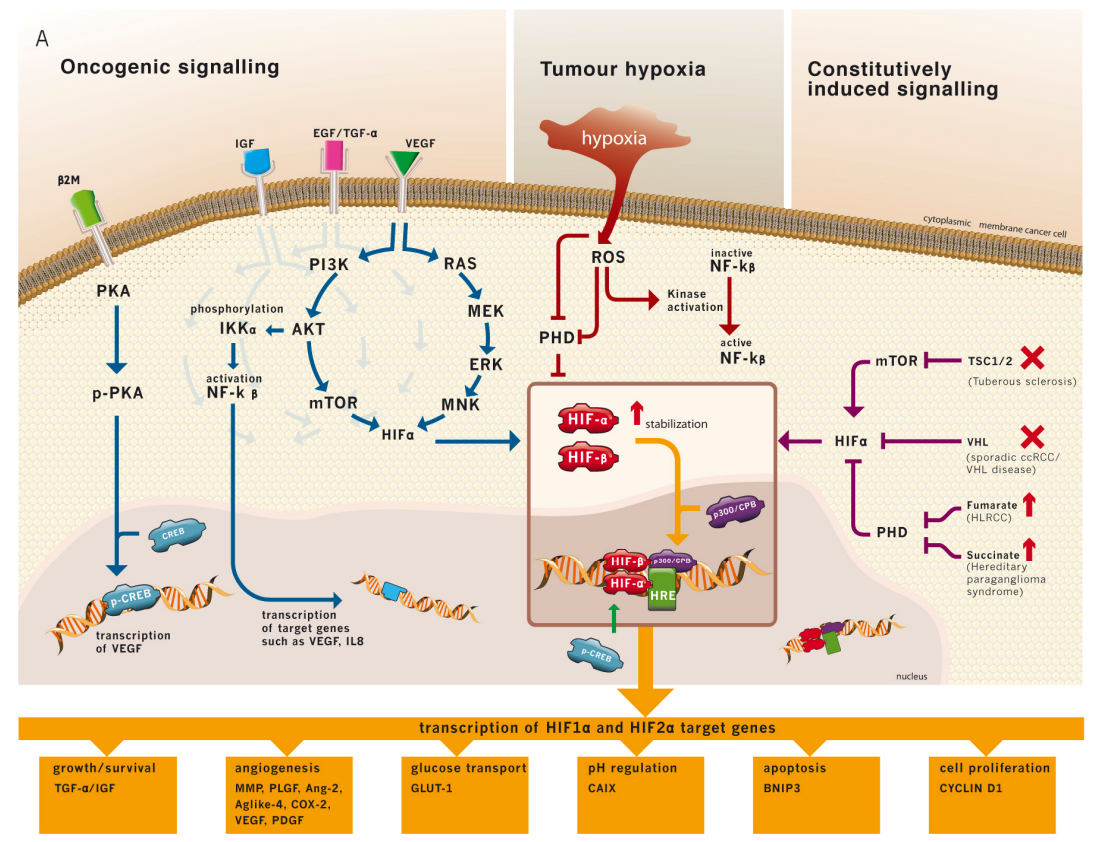

Figure 1A. In ccRCC the HIF pathway can be activated by three main mechanisms: oncogenic signalling (1), hypoxia (2) and constitutively induced signalling (3).

(1). Binding of a ligand to its receptor results in stimulation of several pathways, including the mitogenactivated protein kinase (MAPK:RAS/MNK) and phosphatidylinositol 3- OH kinase (PI3K/AKT) pathways, both promoting translation and accumulation of HIF- $\alpha$. In addition, activation of EGFR/P13K/AKT/IKK $\alpha$ / NF$\mathrm{KB}$ signalling cascade, by TGF $\alpha$, can induce expression of proangiogenic factors such as interleukin-8 (IL-8) and VEGF. $\beta 2$-microglobulin can activate cyclic-AMP-response-element-binding protein (CREB), through cAMP-dependent Proteine Kinase A (PKA) signalling, which in turn may lead to VEGF expression. In the nucleus, HIF- $1 \alpha$ can also interact with transcription factor CREB to activate transcription.

(2). HIF degradation is dependent on hydroxylation of conserved proline residues via enzymes referred to as HIF prolyl hydroxylases (PHD). Molecular oxygen (O2) is required as a cosubstrate for PHD activity. Once hydroxylated, HIF is recognized by the Von Hippel-Lindau (VHL) protein complex and undergoes oxygendependent degradation. Tumour-associated hypoxia inhibits HIF degradation by inhibiting PHD through tricarboxylic acid cycle generation of reactive oxygen species (ROS). HIF- $\alpha$ accumulates and one of the two isoforms of HIF- $\alpha$ (HIF-1 $\alpha$ or HIF-2 $\alpha$ ) then translocates to the nucleus and dimerizes with HIF- $\beta$. The HIF$\alpha /$ HIF- $\beta$ complex binds to hypoxia response elements (HRE) within the promoters of target genes and thereby regulates transcription of genes involved in cell growth, angiogenesis, anaerobic glucose metabolism, $\mathrm{pH}$ regulation, cell survival/apoptosis, cell proliferation and other genes that modulate various cellular functions. Transcriptional co-activators are p300/CBP.

Changes in the redox potential in hypoxic cells due to the generation of ROS in mitochondria can also result in NF-KB activation.

(3). HIF- $\alpha$ can also accumulate via activation of other constitutively induced mechanisms. Loss of Tuberous sclerosis complex 1 or 2 (TSC1 or 2) function, promotes mammalian target of rapamycin (mTOR)-dependent translation and accumulation of HIF alpha in the cells.

In addition, inactivation of $\mathrm{VHL}$ results in stabilization and accumulation of $\mathrm{HIF-} \alpha$. Accumulation of fumarate (as described in hereditary leiomyomatosis renal-cell cancer syndrome (HLRCC)) or accumulation of succinate (hereditary paraganglioma syndrome) causes accumulation of HIF- $\alpha$, through inhibition of PHD.

TGF $\alpha=$ transforming growth factor $\alpha$; IGF = insulin-like growth factor; MMP = matrix metalloproteinase; PLGF = placental growth factor; Ang-2 = angiopoietin-2; Aglike-4 = angiopoietin-like 4; COX-2 = Cyclooxygenase-2; VEGF = vascular endothelial growth factor; PDGF = plateled-derived growth factor; GLUT-1 = glucose transporter-1; CAIX = Carbonic anhydrase IX; BNIP3 = BCL2/adenovirus E1B 19kDa interacting protein 3; $\mathrm{EGF}=$ epidermal growth factor; $\mathrm{NF}-\mathrm{KB}=$ nuclear factor- $\mathrm{KB} ; \mathrm{IKK} \alpha=I$ kappa $\mathrm{B}$ kinase alpha; $\mathrm{AKT}=$ serine/threonine kinases AKT; MEK = mitogen and extracellular kinase; ERK = extracellular receptor kinase; MNK = MAPK-interacting protein kinase. 


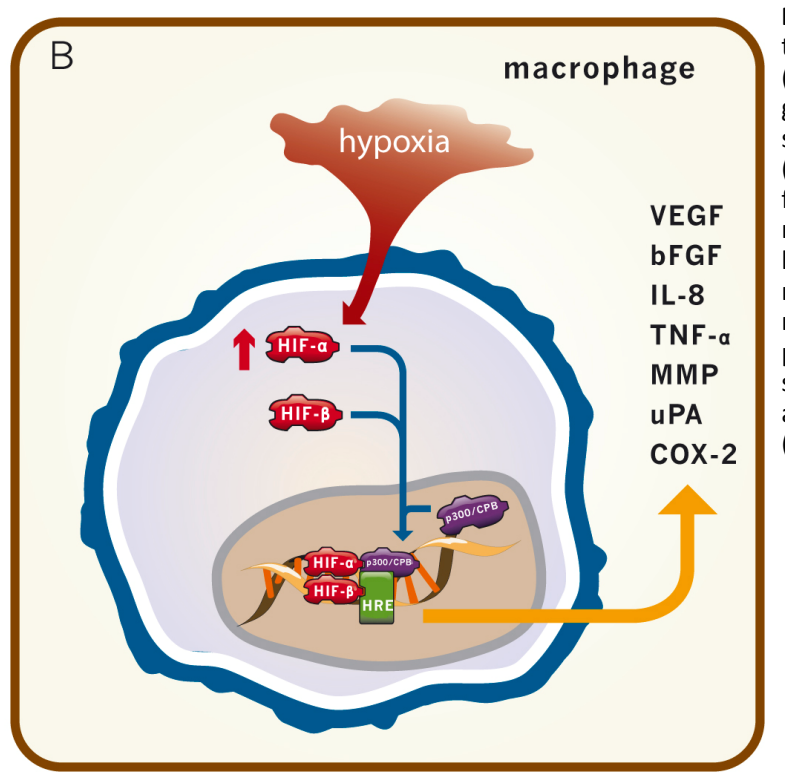

Figure 1B. Pro-angiogenic signalling in tumour-associated macrophages (TAM). A number of potent proangiogenic cytokines and growth factors, such as VEGF, tumour necrosis factor- $\alpha$ (TNF- $\alpha$ ), interleukin-8 (IL-8) and basic fibroblastic growth factor (bFGF) are released by TAMs in response to hypoxia, next to angiogenesismodulating enzymes such as matrix metalloproteinases (MMP) and other proteins that influence angiogenesis uch as urokinase-type plasminogen activator (UPA) and cyclooxygenase-2 (COX-2).

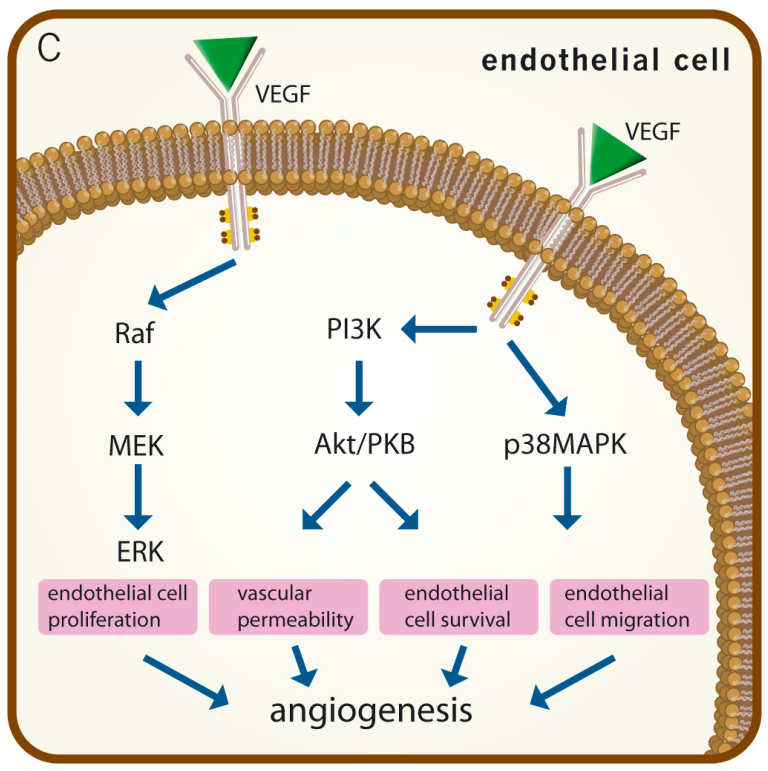

Figure 1C. Overview of the VEGF signalling transduction pathway in the endothelial cell. VEGF binding to a cognate receptor kinase (VEGFR1-2) activates the phosphatidylinositol 3 kinase (PI3K) and mitogen-activated protein kinase (MAPK) pathways. PI3K activates the downstream serine/threonine kinases AKT (also known as protein kinase $B(P K B)$ ) and mammalian target of rapamycin (mTOR).In the MAPK pathway, the extracellular signal-regulated kinase (ERK) is activated by the upstream MAP/ERK kinase (MEK). These signalling pathways result in stimulation of angiogenesis. 

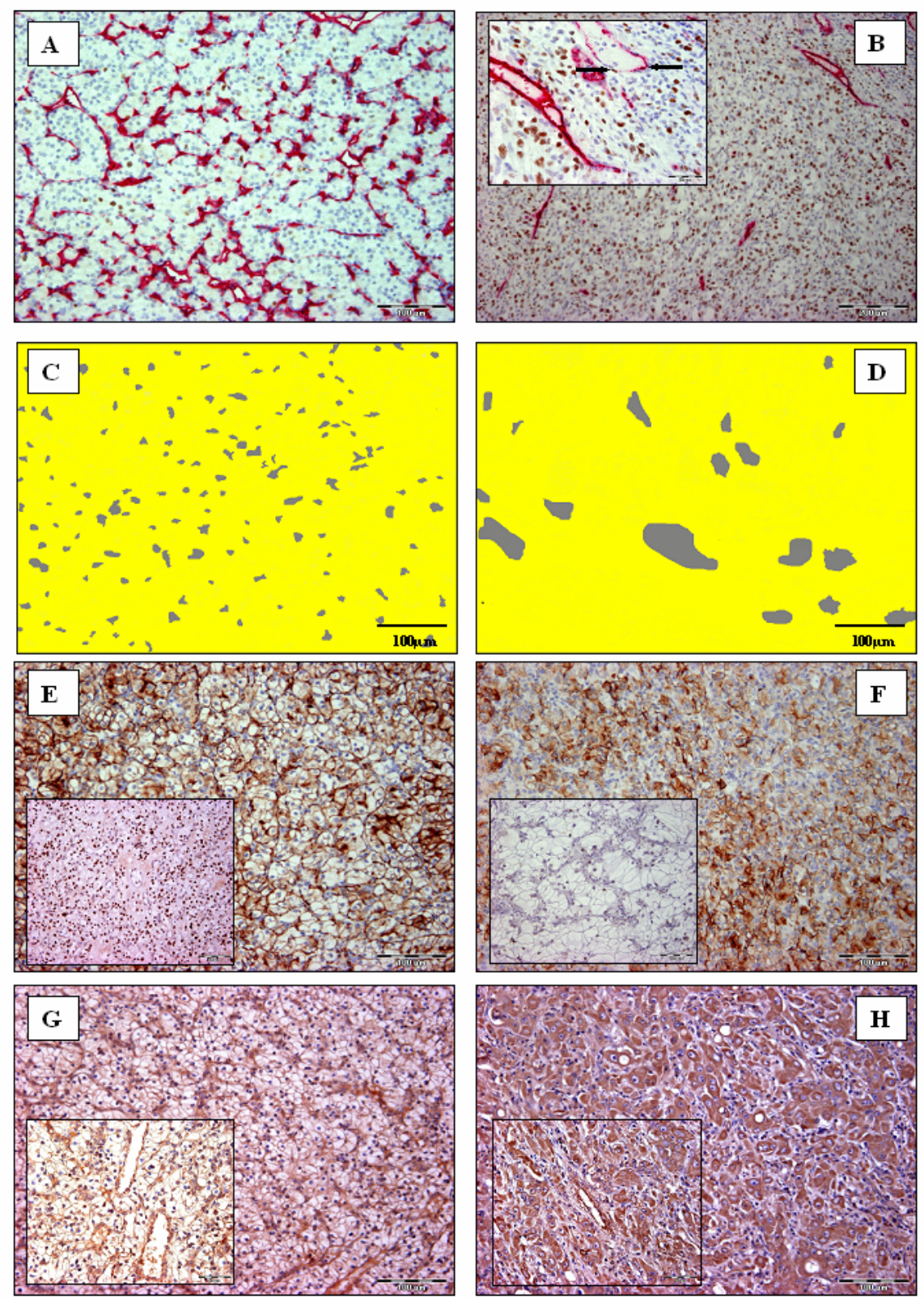

Figure 1: Overview of the immunohistochemical stainings used, in low grade (left: $A, C, E, G$ ) and high grade (right: B,D,F,H) ccRCC. A and B: CD34/Ki-67 IHC double-staining. Black arrows in inset show proliferating Ki67 positive (brown nucleus) endothelial cells (red cytoplasm). C and D: Morphometrical analyses showing an increased vessel area (grey) in high grade ccRCC. E and F : Membranous CA9 staining and nuclear HIF-1 $\alpha$ staining (inset) of tumour cells. Although no significant difference was found between low and high grade tumours for CA9 expression, there was more HIF-1 $\alpha$ expression in low grade ccRCC. $G$ and $\mathrm{H}$ : predominantly membranous VEGF staining in tumour cells of low grade RCC (G), in contrast with dens cytoplasmatic intratumoural VEGF staining in high grade RCC (H). Insets illustrate also VEGF immunoreactivity of the intratumoural vessels. 

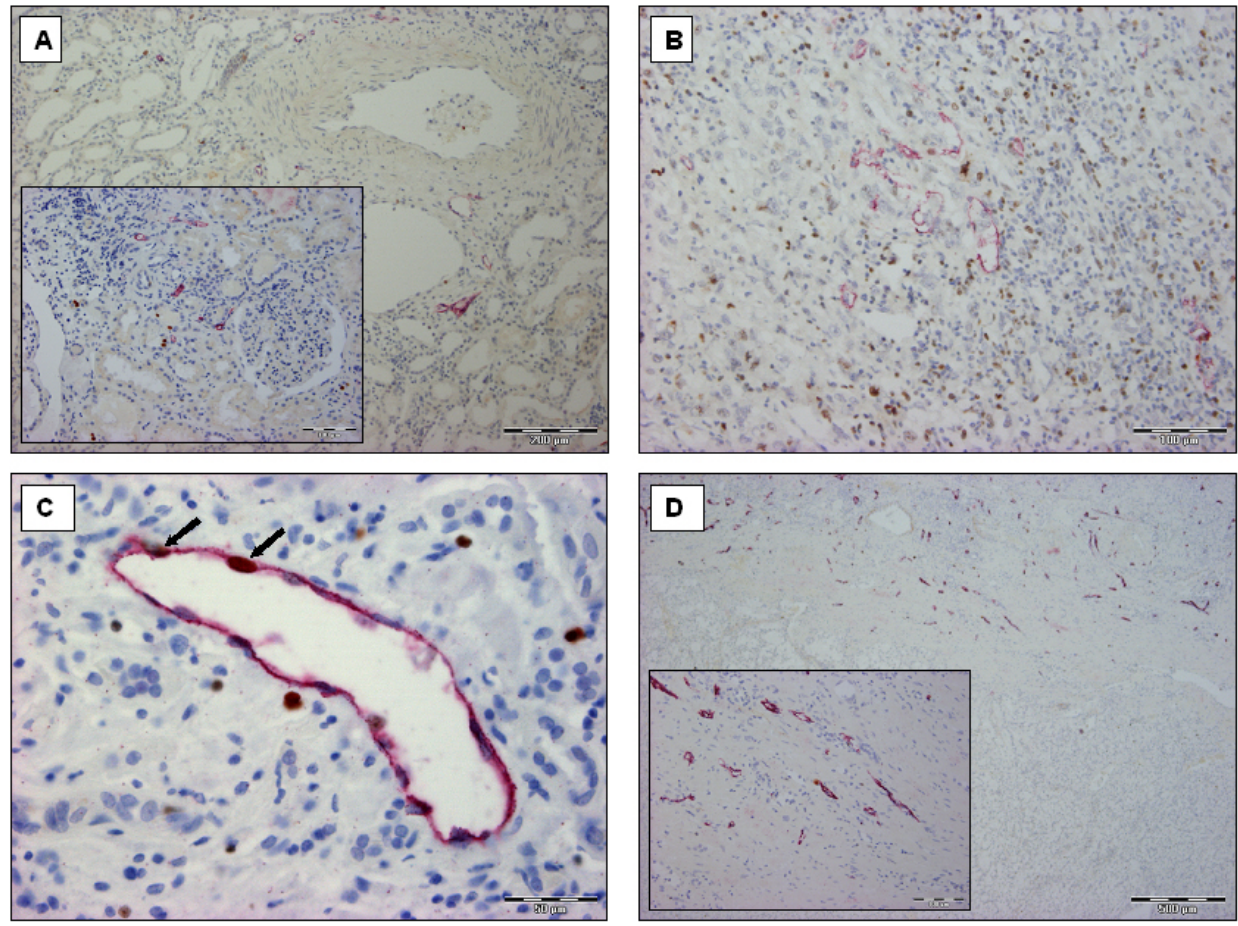

Figure 1: Overview of the D2-40/Ki-67 double immunostainings.

A: non-neoplastic renal tissue with the presence of lymph vessels (red) around intrarenal veins and arteries. Inset illustrates lymphatic capillaries in the neighbourhood of a glomerulus, in the background of an inflammatory interstitium of the tumor-free renal tissue. B: Intratumoural lymph vessels were observed especially in inflammatory areas. C: Black arrows show proliferating Ki-67 positive (brown nucleus) endothelial cells. D:

Hot spots of peritumoural lymph vessels were present in the peritumoural fibrous capsule. Lymphatic capillaries were often irregularly shaped and collapsed (inset). 


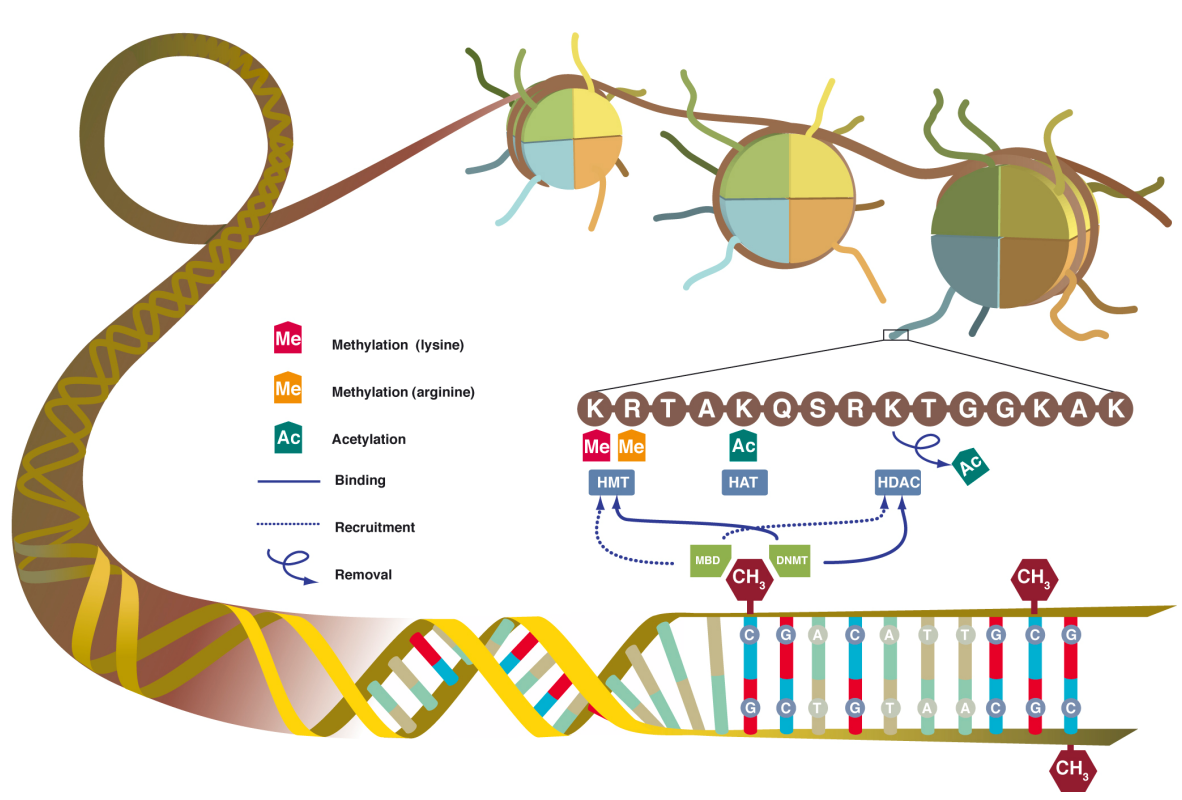

Figure. 1:. Epigenetic modifications and their interplay

The two main epigenetic hallmarks are postreplicative DNA methylation and posttranslational histone modifications, which both play a role in gene silencing and interact to repress gene transcription. (Additional histone modifications are known but not depicted here; histone methylation and acetylation are the most understood.)

DNA methylation is catalyzed by DNA methyltransferases (DNMTs) and characterized by the addition of a methyl group $(\mathrm{CH} 3)$ to a cytosine preceding a guanine $(\mathrm{CpG})$. Histone (de)acetylation results from an imbalance between histone deacetylases (HDACs) and histone acetyltransferases (HATs), whereby deacetylation is associated with gene repression. HDACs and histone methyltransferases (HMTs) are recruited by methylbinding proteins (MBPs), which bind methylated $\mathrm{CpGs}$, or are directly bound to DNMTs to epigenetically modify histone tail residues and regulate gene expression. 


\section{Dankwoord}

"Wanneer je in het leven iets wil bereiken, moet je eerst beginnen met in jezelf te geloven".

Deze uitspraak heb ik gaandeweg in mijn Maastrichtse loopbaan ervaren en leren waarderen.

Ik moet eerlijk bekennen dat ik met een heel klein hartje op weg ging in dit voor mij onbekend wetenschappelijk avontuur. De combinatie met mijn drukke klinische taken en gezinsleven maakte dit zeker niet evident.

Het is uiteindelijk een tocht geworden van vallen en opstaan, waarbij elke tegenslag ook weer een leerschool was in volharding en iedere geslaagde publicatie een welkome opsteker.

Deze weg was ook niet mogelijk geweest zonder de hulp van vele anderen, die ik hierbij even wil bedanken.

Promotor Prof. Dr. Adriaan de Bruïne, beste Adriaan, je was voor mij een geweldige begeleider en collega. Onze overlegmomenten inspireerden mij enorm in het schrijven van artikels en bij tegenslagen wist je me altijd weer aan te moedigen. Het was voor mij rustgevend dat ik op jouw steun kon rekenen, niet enkel in de wetenschap, maar ook in de klinische taken. Ik was je erg dankbaar als je op piekmomenten mijn uropraktijkje wou overnemen, zodat ik aan mijn onderzoek kon werken. Jammer genoeg, is er een einde gekomen aan onze professionele samenwerking. Ik zal je als mens en als collega missen. Veel geluk in je nieuwe loopbaan!

Copromotor Dr. Manon van Engeland, beste Manon, ik ben je heel erg dankbaar dat ik destijds bij jouw onderzoeksgroep kon komen en dat je mij ten volle in mijn onderzoekstraject gesteund en begeleid hebt. Epigenetica was werkelijk een nieuwe wereld die voor mij openging. Je hebt een fijne onderzoeksgroep, die je geweldig aanstuurt en waar de sfeer goed zit.

Copromotor Dr. Peter Vermeulen, beste Peter, ik herinner mij nog zeer goed het moment dat ik, vertoevend in een serieuze onderzoeksdip, bij jou aanklopte. Je was meteen luisterbereid en bood mij nieuwe ideeën en onderzoeksmiddelen aan binnen je eigen labo, waarvoor ik je nog steeds heel erg dankbaar ben. Ik kan je niet beschrijven hoe gelukkig ik was toen ons eerste artikel aanvaard werd voor publicatie. 
Ik zou ook graag alle co-auteurs, die hun steentje bijgedragen hebben aan de artikels en ook iedereen van de angiogenese groep in Maastricht en Antwerpen, die een bijdrage geleverd hebben aan mijn onderzoek, willen bedanken. Hierbij wil ik vooral Gert in de bloemetjes zetten. Zijn efficiënte aanpak en hulp hebben mij destijds heel erg vooruit geholpen.

Vervolgens is er ook nog de epigenetica onderzoeksgroep, waar ik vooral Fiona, Kim en zeker Iris even wil aanhalen. Fiona, bedankt voor alle ondersteuning in het lab. Kim, ik was heel blij dat je je wou ontfermen over de statistische kant van het onderzoek. Iris, je was voor mij een grote steun en hulp en samen met jou een review schrijven was een spannend avontuur met een geweldig einde. Het nieronderzoek is bij jou in goede handen en ik hoop dat we nog lang kunnen samenwerken. Daarenboven kijk ik er al naar uit om jou, als paranimf, aan mijn zijde te hebben.

Mat Daemen die, tot mijn grote vreugde toestemde om zich te ontplooien tot mijn andere paranimf, ben ik ook in vele opzichten dankbaar. Mat, het feit dat ik op je steun en begeleiding vooral bij de start van mijn onderzoek kon rekenen en je onafgebroken vertrouwen in mijn kunnen, gaven mij de nodige ruggesteun. Je "peptalks" werkten inspirerend!

Mijn collega-pathologen wil ik bedanken dat ze mij gedurende die zes jaren ruimte gegeven hebben om mijn onderzoek waar te maken. Ik kon altijd rekenen op hun steun en hulp in klinisch werk bij moeilijke momenten.

Ik zou het harde werken nooit volgehouden hebben zonder mijn gedreven passie voor de muziek. Elke letter van dit proefschrift werd geschreven met rustgevende en inspirerende pianomuziek op de achtergrond. Dankzij het enthousiasme van Jos, mijn pianoleraar, kon ik mij uitleven in de pianoconcerto's van Haydn en Grieg.

Verder zou ik zonder de hulp en steun van mijn ouders en schoonouders mijn medische carrière nooit op een dergelijk peil hebben kunnen uitbouwen.

Bedankt dat jullie altijd voor me klaarstonden! 
Robin en Lindsey, mijn twee schatten van kinderen, dank voor jullie begrip als mama weer eens een weekendje haar bureauwerk moest trotseren. Jullie spontane hulp in huishoudelijke taken en interesse hebben mij erg verrast. Robin, ik vond het geweldig dat je mee op zoek ging naar een mooie frontpagina voor mijn boekje. Lindsey, je plagende snoet deed me telkens weer lachen.

And... last but not least, is er mijn lieve echtgenoot en "mijn rots in de branding". Johan, zonder jou had ik het niet gered! Ondanks je drukke huisartsenpraktijk, steunde je mij door dik en dun. Je gevatte humor en je latino danspassen zijn de bubbels in mijn leven! 


\section{About the author}

Marcella Baldewijns was born on May $10^{\text {th }} 1966$ in Herk-de-Stad, Belgium. In 1984 she graduated from St. Ursula high school in Herk-de-stad. From 1984 till 1991 she studied Medicine at the universities of Diepenbeek (three Bachelor years) and Leuven (four Master years). In July 1991, she started as a resident at the department of Pathology, University Hospital of Leuven. After completing her Pathology training in 1997, she worked as a Pathologist at the Vesalius Hospital of Tongeren for three years. Afterwards, she was affiliated with the Pathology Department of the Catharina Hospital in Eindhoven and Maxima Medical Center of Veldhoven. In May 2003 she started to work as a staff member of the Pathology Department at the Maastricht University Medical Center, where she investigated angiogenesis and the role of epigenetics in Renal Cell Carcinoma. As of June 2009, her post doctoral research consists of further epigenetical studies on Renal Cell Carcinoma in collaboration with a research-AIO Iris van Vlodrop, who started to work with her in February 2007. 


\section{List of Publications}

van de Voorde W, Baldewijns M, Lauweryns J (1994) Florid basal cell hyperplasia of the prostate. Histopathology 24: 341-8

Baldewijns M, Ectors N, Verbeeck G, Janssens J, De Schepper J, Ponette E, Geboes K, Desmet V (1995) Intermittent subobstruction and cholestasis as complications of duodenal amyloid tumours. Gastroenterol Clin Biol 19: 218-21

Duin LK, Marcus-Soekarman D, Baldewijns MM, Robben SG, Nijhuis JG (2006) Prenatal diagnosis of bronchial atresia, early in pregnancy. Prenat Diagn 26: 373-6

Baldewijns MM, Thijssen VL, Van den Eynden GG, Van Laere SJ, Bluekens AM, Roskams T, van Poppel H, De Bruine AP, Griffioen AW, Vermeulen PB (2007) High-grade clear cell renal cell carcinoma has a higher angiogenic activity than low-grade renal cell carcinoma based on histomorphological quantification and qRT-PCR mRNA expression profile. Br J Cancer 96: 1888-95

Duin LK, Willekes C, Baldewijns MM, Robben SG, Offermans J, Vles J (2007) Major brain lesions by intrauterine herpes simplex virus infection: MRI contribution. Prenat Diagn 27: 81-4

M.M.L Baldewijns, I.J.H van Vlodrop, L.J. Schouten, P.M.M.B. Soetekouw, A.P. de Bruïne and M. van Engeland (2008) Genetics and epigenetics of renal cell cancer, Biochimica et Biophysica Acta 1785, 133-155

Baldewijns MM, Roskams T, Ballet V, Van den Eynden GG, Van Laere SJ, Van der Auwera I, Lerut E, De Bruïne AP, Thijssen VL, Vermeulen PB en van Poppel H (2009) Low frequency of lymph node metastasis in clear cell renal cell carcinoma is related to low lymphangiogenic potential. BJU International 2009, Epub ahead of print.

Marcella M. Baldewijns, Iris J.H. van Vlodrop, Peter B. Vermeulen, Patricia M.M.B. Soetekouw, Manon van Engeland and Adriaan P. de Bruïne. The central role of HIF signalling in Renal Cell Carcinoma angiogenesis: implications for prognosis and therapy. Submitted for publication in 2009. 
Marcella M Baldewijns, Iris JH van Vlodrop, Kim M Smits, Peter B Vermeulen, Gert G Van den Eynden, Fiona Schot, Tania Roskams, Hein van Poppel, Manon van Engeland and Adriaan P de Bruïne. Different angiogenic potential in low and high grade sporadic clear cell carcinoma is not related to alterations in the von Hippel-Lindau gene. Cellular Oncology 2009, in press.

Iris J.H. van Vlodrop, Marcella M.L. Baldewijns, Kim M. Smits, Leo J. Scouten, Leander van Neste, Wim van Criekinge, Hein van Poppel, Evelyne Lerut, Kornel Schuebel, Nita Ahuja, James J. Herman, Adriaan de Bruïne, and Manon van Engeland. Prognostic significance of Gremlin 1 (GREM1) promoter CpG island hypermethylation in clear cell renal cell carcinoma. Submitted for publication in 2009.

Dean Troyer, M.Scott Lucia, Adriaan de Bruïne, Rosario Mendez-Meza, Marcella Baldewijns, Nancy Dunscomb, Manon van Engeland, Theresa mcAskill, Katja Bierau, Joost Louwagie, Joseph Bigley. Prostate Cancer Detected by Methylated Gene Markers in Histopathologically Cancer-Negative Tissues from Men with Subsequent Positive Biopsies. Submitted for publication in 2009. 